US Army Corps of Engineers ${ }_{\circledast}$

Engineer Research and

Development Center

\title{
Laboratory Characterization of Alkalf- Silica Reaction Susceptibility of Aggregates for Charleroi Lock and Dam, Monongahela River Project
}

Monica A. Ramsey

December 2019

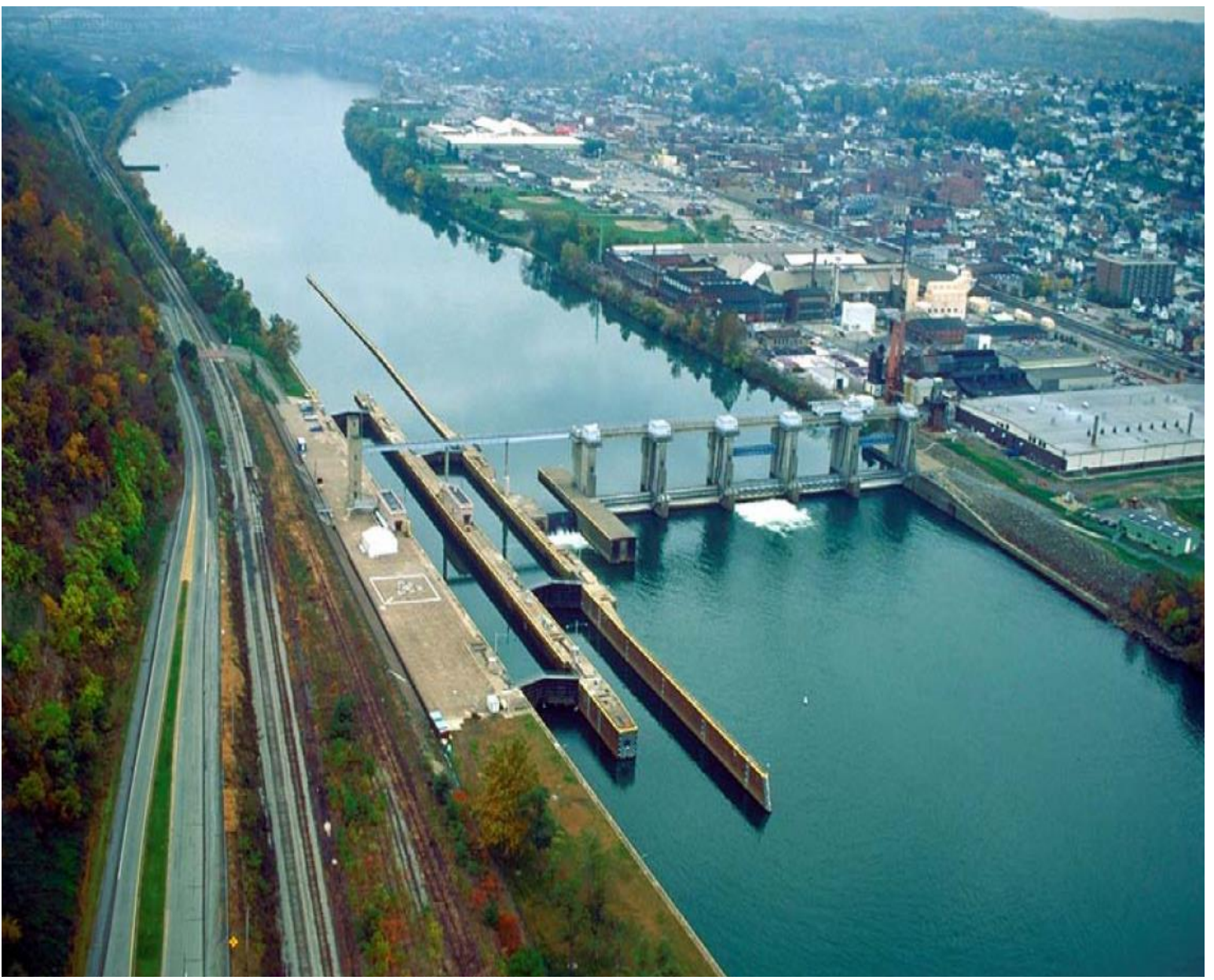


The U.S. Army Engineer Research and Development Center (ERDC) solves the nation's toughest engineering and environmental challenges. ERDC develops innovative solutions in civil and military engineering, geospatial sciences, water resources, and environmental sciences for the Army, the Department of Defense, civilian agencies, and our nation's public good. Find out more at www.erdc.usace.army.mil.

To search for other technical reports published by ERDC, visit the ERDC online library at http://acwc.sdp.sirsi.net/client/default. 


\section{Laboratory Characterization of Alkali-Silica Reaction Susceptibility of Aggregates for Charleroi Lock and Dam, Monongahela River Project}

Monica A. Ramsey

Geotechnical and Structures Laboratory

U.S. Army Engineer Research and Development Center 3909 Halls Ferry Road

Vicksburg, MS 39180-6199

Final report

Approved for public release; distribution is unlimited.

Prepared for U.S. Army Corps of Engineers Pittsburgh District

Pittsburgh, PA 15222

Under MIPR W81ET491842462, Lower Monongahela River Navigation System Feasibility Study 


\section{Abstract}

The purpose of this study was to identify potential alkali-aggregate reactivity of local aggregates provided by the Pittsburgh District according to ASTM C1260 and to investigate the effectiveness of a combination of cementitious materials and aggregates from provided mixture designs in suppressing alkali-silica reactivity (ASR) induced expansions according to the ASTM C1567. Three ASTM C1260 tests were performed for each aggregate (Hanson, Georgetown, and Shelly). A total of 48 ASTM C1567 tests were performed from the combination of the four mix designs, four fly ash sources, and three aggregate sources. The limiting criteria for the proposed materials and mix designs was expansions less than $0.08 \%$ at 30 days of testing in accordance to the Unified Facilities Guide Specification (UFGS) Division 03 Concrete Section 0330 00.50. Based on this specification, the tested aggregates are considered potentially reactive with 30-day mortar bar expansions of $0.1970 \%$ for Hanson, $0.1683 \%$ for Georgetown, and $0.1623 \%$ for Shelly. However, all 48 combinations of the ASTM C1567 tests passed the limiting criteria with 30-day expansions less than $0.08 \%$. These results indicate that the constituent project materials in the proposed mix designs can effectively mitigate ASR.

DISCLAIMER: The contents of this report are not to be used for advertising, publication, or promotional purposes. Citation of trade names does not constitute an official endorsement or approval of the use of such commercial products. All product names and trademarks cited are the property of their respective owners. The findings of this report are not to be construed as an official Department of the Army position unless so designated by other authorized documents.

DESTROY THIS REPORT WHEN NO LONGER NEEDED. DO NOT RETURN IT TO THE ORIGINATOR. 


\section{Contents}

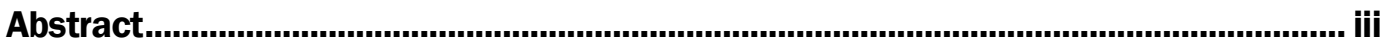

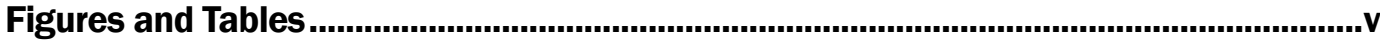

Preface

1 Introduction

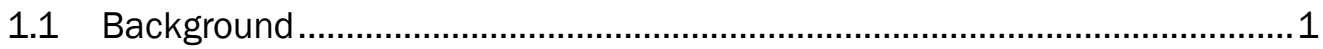

1.2 Problem statement ........................................................................... 1

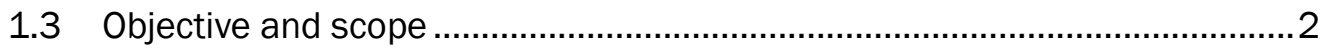

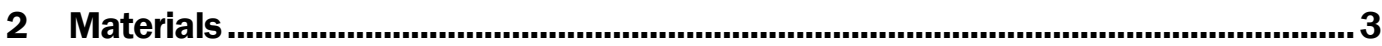

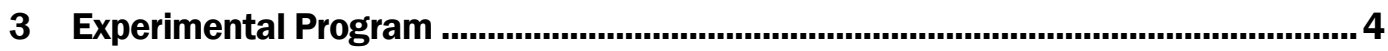

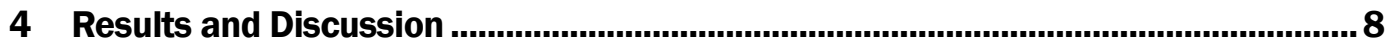

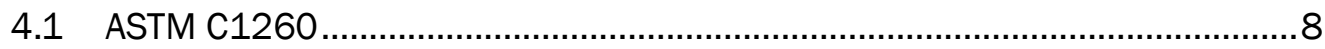

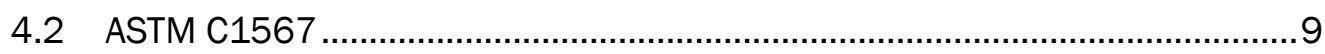

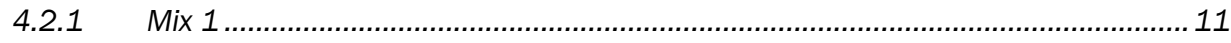

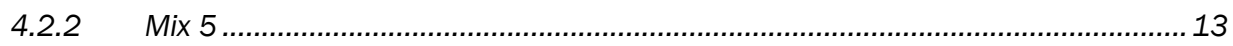

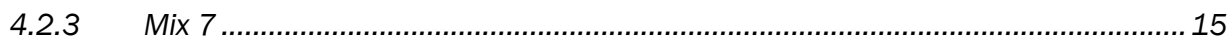

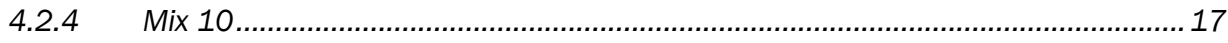

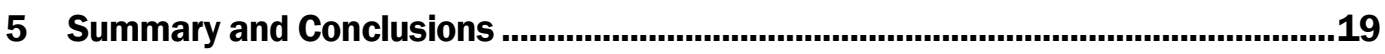

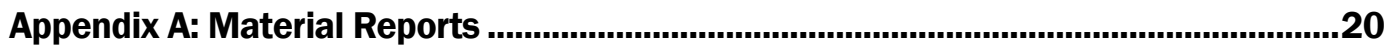

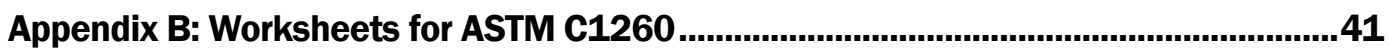

Appendix C: Worksheets for ASTM C1567 ................................................................45

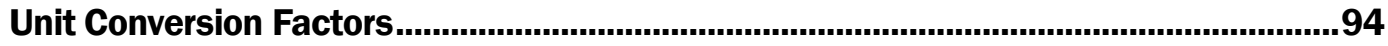

Report Documentation Page 


\section{Figures and Tables}

\section{Figures}

Figure 1. Mortar bar expansions of Hanson, Georgetown, and Shelly aggregates in ASTM C1260.

Figure 2. Mortar bar expansions versus time for Mix 1.......................................................12

Figure 3. Mortar bar expansions versus time for Mix 5.......................................................14

Figure 4. Mortar bar expansions versus time for Mix 7....................................................16

Figure 5. Mortar bar expansions versus time for Mix 10 ................................................18

\section{Tables}

Table 1. Summary of mix designs, aggregate sources, and fly ash sources used in

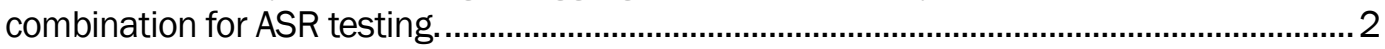

Table 2. Summary of materials used in ASR testing........................................................ 3

Table 3. Concrete mixture proportions for Charleroi L\&D. .................................................... 5

Table 4. Aggregate gradation for testing by ASTM C1260/C1567...................................... 6

Table 5. Batch proportions used to prepare mortar bars for ASTM C1567........................... 6

Table 6. Average length change percent of all project aggregates in ASTM C1260. .......... 8

Table 7. Results of all ASTM C1567 tests at 30 days........................................................10

Table 8. Average length change of mortar bars made from Mix 1 materials.....................11

Table 9. Average length change of mortar bars made from Mix 5 materials.....................13

Table 10. Average length change of mortar bars made from Mix 7 materials....................15

Table 11. Average length change of mortar bars made from Mix 10 materials. .................17 


\section{Preface}

This study was conducted by U.S. Army Engineer Research and Development Center (ERDC) for the Corps of Engineers Pittsburgh District in Pittsburgh, PA, for Charleroi Lock Reconstruction project under MIPR W81ET491842462. The Pittsburgh District technical manager was Mr. Glenn W. Bush Jr.

The work was performed by the Concrete and Materials Branch (GMC) of the Engineering Systems and Materials Division (GM), U.S. Army Engineer Research and Development Center, Geotechnical and Structures Laboratory (ERDC-GSL). At the time of publication, Mr. Christopher M. Moore was GMC Chief; Mr. Justin S. Strickler was GM Chief; and Mr. R. Nicholas Boone was the Technical Director for Force Projection and Maneuver Support. The Deputy Director of ERDC GSL was Mr. Charles W. Ertle II, and the Director was Mr. Bartley P. Durst.

COL Teresa A. Schlosser was the Commander of ERDC, and Dr. David W. Pittman was the Director. 


\section{Introduction}

\subsection{Background}

The baseline design for the Charleroi Locks originated in 1991 from the Lower Monongahela River Navigation System Feasibility Study. The feasibility study evaluated the ability of Locks and Dams 2, 3, and 4 of the Monongahela River Navigation System to serve navigation interests through the year 2050. The conclusion of the study was to implement a "2 for 3 " replacement known as "The Lower Mon Project."

The Lower Mon Project consists of replacement of the fixed-crest dam at Locks and Dam 2 with a gated dam to be renamed the Braddock Locks and Dam, construction of twin 84-ft by 720 -ft locks at Locks and Dam 4 to be renamed the Charleroi Locks and Dam, removal of Locks and Dam 3 at Elizabeth, navigation dredging, and relocation of adversely impacted public facilities. The combination of these features will allow for one navigation pool between Braddock Locks and Dam and the Charleroi Locks and Dam, i.e., a 30.3-mile pool.

The Charleroi Lock reconstruction began in 2002 with site development and has continued as funding has become available for separate contracts for the construction of the river wall, upper and lower guide walls, emptying basin, middle wall monoliths M22-M27, and river chamber completion. The project uses government-designed concrete mixes developed in 2005 by the U.S. Army Engineer Research Development Center (ERDC) in Vicksburg, MS, and produced at the left bank batch plant, a government-owned, contractor-operated batch plant located across the river from the lock chambers.

\subsection{Problem statement}

Alkali-silica reaction (ASR) is a chemical reaction between alkali hydroxides in concrete and active minerals in aggregates that can cause serious expansion and cracking in concrete. The premature distress can result in loss in serviceability in structures such as locks and dams. It is generally recognized that the use of a sufficient quantity of a suitable supplementary cementitious materials (SCM) is one of the more efficient preventative measures for controlling expansion. 
Standard test methods to evaluate the ASR susceptibility of aggregates and potential methods to mitigate ASR were performed by ERDC in 2005. However, the material quality tests were outdated, and some original source suppliers of fly ash were no longer available. To ensure all local materials met the quality requirements of the Lower Mon Project specifications, the Pittsburgh District re-commissioned the materials testing for validation.

\subsection{Objective and scope}

The principal objective of the testing was to determine the potential reactivity of three aggregates and the effectiveness in suppressing ASRinduced expansion using a combination of SCMs in mixture designs provided by the District. Such materials include class F fly ash, silica fume, and ground-granulated blast furnace slag. Testing was performed in accordance to ASTM C126o, Standard Test Method for Potential Alkali Reactivity of Aggregates (Mortar-Bar Method), and ASTM C1567, Standard Test Method for Determining the Potential Alkali-Silica Reactivity of Combinations of Cementitious Materials and Aggregate (Accelerated Mortar-Bar Method). A total of 48 tests were performed using the combination of mix designs, aggregates, and fly ash sources summarized in Table 1. The purpose was to ensure that the mixture design adequately mitigated expansions due to ASR in all possible combinations.

Table 1. Summary of mix designs, aggregate sources, and fly ash sources used in combination for ASR testing.

\begin{tabular}{|c|c|c|}
\hline Mix Design & Aggregate Source & Fly Ash Source \\
\hline Mix 1 & Hanson CA & Sammis \\
\hline Mix 5 & Georgetown FA & Ft. Martin \\
\hline Mix 7 & Shelly FA & Longview \\
\hline Mix 10 & & Brandon Shores \\
\hline
\end{tabular}




\section{Materials}

The materials listed in Table 2 were provided by the USACE-Pittsburgh District for testing described in this report. ERDC tested all materials in accordance to the ASTM requirement reference in the table for validation prior to ASR testing. Results are provided in Appendix A.

Table 2. Summary of materials used in ASR testing.

\begin{tabular}{|c|c|c|c|c|}
\hline Material & Manufacturer & Location & CMB \# & $\begin{array}{c}\text { Testing } \\
\text { Validation }\end{array}$ \\
\hline Type II(MH) Cement & Armstrong & Cabot, PA & 150063 & ASTM C150 \\
\hline \multirow{4}{*}{ Class F Fly Ash } & $\begin{array}{l}\text { Headwaters } \\
\text { Resources, } \\
\text { Ft. Martin }\end{array}$ & Maidsville, WV & 150064 & \multirow{4}{*}{ ASTM C618 } \\
\hline & $\begin{array}{l}\text { Headwaters } \\
\text { Resources, } \\
\text { W. H. Sammis }\end{array}$ & Stratton, $\mathrm{OH}$ & 150065 & \\
\hline & $\begin{array}{l}\text { Separation } \\
\text { Technologies, } \\
\text { Longview }\end{array}$ & Maidsville, WV & 180005 & \\
\hline & $\begin{array}{c}\text { Separation } \\
\text { Technologies, } \\
\text { Brandon Shores }\end{array}$ & Curtis Bay, MD & 180018 & \\
\hline $\begin{array}{l}\text { Slag Cement, } \\
\text { Grade } 100\end{array}$ & $\begin{array}{l}\text { Argos-Essroc- } \\
\text { Lehigh }\end{array}$ & Middlebranch, $\mathrm{OH}$ & 150066 & ASTM C989 \\
\hline Limestone Powder & Graymont & Bellefonte, PA & 150067 & ASTM D242 \\
\hline Silica Fume & Elkem Materials & Pittsburgh, PA & 150068 & ASTM C1240 \\
\hline Coarse Aggregate & Hanson & Connellsville, PA & 150070 & $\begin{array}{l}\text { ASTM C40 } \\
\text { ASTM C88 }\end{array}$ \\
\hline \multirow{2}{*}{ Fine Aggregate } & Georgetown & Georgetown, PA & 150069 & \multirow{2}{*}{$\begin{array}{c}\text { ASTM } \\
\text { C127/128 } \\
\text { ASTM C131 } \\
\text { ASTM C136 }\end{array}$} \\
\hline & Shelly & Reedsville, $\mathrm{OH}$ & 160004 & \\
\hline AEA-92 Admixture & \multirow{5}{*}{ Euclid } & \multirow{5}{*}{ Cleveland, $\mathrm{OH}$} & 150073 & \multirow{5}{*}{ ASTM C494 } \\
\hline RET 75 Admixture & & & 150074 & \\
\hline WR Admixture & & & 150075 & \\
\hline AW Admixture & & & 150076 & \\
\hline HRWR Admixture37 & & & 150077 & \\
\hline
\end{tabular}




\section{Experimental Program}

Expansion-based measurements of ASR damage are by far the most common test methods used to assess the ASR susceptibility of aggregates. The potential reactivity of the three project aggregates (Hanson, Georgetown, and Shelly) was determined according to the ASTM C1260, Standard Test Method for Potential Alkali Reactivity of Aggregates (Mortar-Bar Method).

In a similar test, the efficiency of suppressing ASR-induced expansion using a combination of SCM's was determined according to the ASTM C1567, Standard Test Method for Determining the Potential Alkali-Silica Reactivity of Combinations of Cementitious Materials and Aggregate (Accelerated Mortar-Bar Method). The combination of SCMs was based on the composition of the four concrete mixture proportions, i.e., Mix 1, 5, 7, and 10, provided in Table 3 as outlined in the Charleroi project specifications.

The ASTM C1567 test method was developed for a single aggregate source in mortar of standard proportions and currently does not have a method for testing a combination of fine and coarse aggregates as specified in the actual concrete mixture design. To overcome the fixed concrete mixture designs, all three aggregate source materials were tested independently with each fly ash source. A total of 48 tests were performed from the combination of the four mix designs (Mix 1, 5, 7, and 10), four fly ash sources (Sammis, Fort Martin, Longview, and Brandon Shores), and three aggregate sources (Hanson, Georgetown, and Shelly). 
Table 3. Concrete mixture proportions for Charleroi L\&D.

\begin{tabular}{|c|c|c|c|c|}
\hline \multirow[b]{2}{*}{ Material } & Mix 1 & Mix 5 & Mix 7 & Mix 10 \\
\hline & \multicolumn{4}{|c|}{ Batch Quantities (lb/yd³) } \\
\hline Cement (lb/yd) & 167 & 191 & 253 & 495 \\
\hline Class F Fly Ash (Ib/yd) & 158 & 75 & 139 & 165 \\
\hline Slag Cement (Ib/yd) & 367 & 87 & 69 & 0 \\
\hline Silica Fume (Ib/yd) & 29 & 0 & 0 & 0 \\
\hline Limestone Powder (lb/yd) & 155 & 0 & 0 & 0 \\
\hline $3 / 4$ in. Coarse Aggregate (lb/yd) & 1,323 & 739 & 1,129 & 1,720 \\
\hline $\begin{array}{l}11 / 2 \text { in. Coarse Aggregate } \\
(\mathrm{lb} / \mathrm{yd})\end{array}$ & 0 & 747 & 935 & 0 \\
\hline 3 in. Coarse Aggregate (lb/yd) & 0 & 1,000 & 0 & 0 \\
\hline Fine Aggregate (Ib/yd) & 1,409 & 958 & 1,141 & 1,239 \\
\hline Potable Water (Ib/yd) & 303 & 183 & 213 & 258 \\
\hline $\begin{array}{l}\text { Air Entraining Admixture (AEA) } \\
\text { (fl oz/yd) }\end{array}$ & 0 & 8 & 16 & 7 \\
\hline $\begin{array}{l}\text { Water Reducing Admixture } \\
\text { (WRA) (fl oz/yd) }\end{array}$ & 0 & 0 & 0 & 0 \\
\hline $\begin{array}{l}\text { High Range Water Reducing } \\
\text { Admixture (HRWRA) (fl oz/yd) }\end{array}$ & 138.1 & 0 & 0 & 12 \\
\hline $\begin{array}{l}\text { Retarding Admixture (RET) (fl } \\
\text { oz/yd) }\end{array}$ & 30.1 & 7 & 7 & 7 \\
\hline $\begin{array}{l}\text { Anti-Washout Admixture (AWA) } \\
\text { (oz/yd) }\end{array}$ & 97.2 & 0 & 0 & 0 \\
\hline
\end{tabular}

Material quantities were proportioned to yield four 1-in. by 1-in. by 11.25-in. mortar specimens, which is one more than the minimum required. Each batch consisted of $587 \mathrm{~g}$ of cement or cementitious materials and 1,350 $\mathrm{g}$ of aggregate processed to the gradations shown in Table 4. All mortars were prepared with the water-to-cementitious materials ratio maintained at 0.47 by mass. Actual ASTM C1567 batch proportions scaled from the concrete mix designs previously discussed are detailed in Table 5. Due to prolonged setting time, all retarder was removed from the mixture proportion, and test specimens were moist cured $48 \mathrm{hr}$ (opposed to the standard 24-hr moist cure) before demolding. 
Table 4. Aggregate gradation for testing by ASTM C1260/C1567.

\begin{tabular}{|c|c|}
\hline Retained on Sieve & Mass \% \\
\hline No. 8 & 10 \\
\hline No. 16 & 25 \\
\hline No. 30 & 25 \\
\hline No. 50 & 25 \\
\hline No. 100 & 15 \\
\hline
\end{tabular}

Table 5. Batch proportions used to prepare mortar bars for ASTM C1567.

\begin{tabular}{|l|c|c|c|c|c|}
\hline \multirow{2}{*}{ Material } & Mix 1 & Mix 5 & \multicolumn{2}{c|}{ Mix 7 } & Mix 10 \\
\cline { 2 - 6 } & \multicolumn{4}{|c|}{ Mass (g) } \\
\hline Type II(MH) Cement & 136 & 318 & 322 & 440 \\
\hline Class F Fly Ash & 129 & 125 & 177 & 0 \\
\hline Slag Cement & 299 & 144 & 88 & 147 \\
\hline Silica Fume & 23 & 0 & 0 & 0 \\
\hline Limestone Powder & 126 & 0 & 0 & 0 \\
\hline \multicolumn{1}{|c|}{ Admixture } & 0 & 0.87 & 1.33 & 0.41 \\
\hline $\begin{array}{l}\text { Air Entraining Admixture } \\
\text { (AEA) }\end{array}$ & 0 & 0 & 0 & 0.70 \\
\hline $\begin{array}{l}\text { Water Reducing } \\
\text { Admixture (WRA) }\end{array}$ & 5.68 & 0 & 0 & 0 \\
\hline $\begin{array}{l}\text { High Range Water } \\
\text { Reducing Admixture } \\
\text { (HRWRA) }\end{array}$ & 0 & 0 & 0 & 0 \\
\hline $\begin{array}{l}\text { Retarding Admixture } \\
\text { (RET) }\end{array}$ & 5.16 & 0 & 0 & 0 \\
\hline $\begin{array}{l}\text { Anti-Washout Admixture } \\
\text { (AWA) }\end{array}$ & 0 & 0 & 0 & 0 \\
\hline
\end{tabular}

The mortar mixes were prepared in 4 min in accordance with ASTM C3O5 using a 71-L capacity Hobart planetary mixer with a clearance of $5.1 \mathrm{~mm}$ between the lower end of the paddle and the bottom of the bowl. First, the water was added to the bowl, then each admixture was added into the water using a pipette accurate to $1.0 \mu \mathrm{m}$. The liquids were then mixed on 
low until homogeneous. Then, the blended dry cementitious materials were introduced and mixed for $30 \mathrm{sec}$ on low. Next, the test aggregate was slowly added and mixed for $30 \mathrm{sec}$. The mixer was then changed to medium speed and mixed an additional $30 \mathrm{sec}$. The mixer was stopped, then the mixture was scraped with a spatula for $15 \mathrm{sec}$, taking care to mix the bottom of the mortar. This was then allowed to sit for $90 \mathrm{sec}$ with the cover in place. Finally, the cover was removed, and the mortar was mixed for $60 \mathrm{sec}$ at medium speed. All molds were filled by compacting the mixture with a tamper within 2 min and $15 \mathrm{sec}$ after completion of the mixing. The mortar test specimens were stored in a $23^{\circ} \mathrm{C}$ moist cabinet for $48 \mathrm{hr}$ before demolding and making the initial length measurements.

The test specimens were then immersed in sealed plastic containers filled with tap water maintained in a water bath at $80^{\circ} \mathrm{C}$ for $24 \mathrm{hr}$. The zerolength measurement was then taken, and the specimens were transferred in containers filled with a $1-\mathrm{N} \mathrm{NaOH}$ solution at $80^{\circ} \mathrm{C}$ maintained in a water bath at $80^{\circ} \mathrm{C}$ to accelerate expansion. Expansion measurements were made using a length comparator accurate to $1.0 \mu \mathrm{m}$ at $3,5,7,14,21$, 28 , and 30 days, which is more than twice as long as the standard test duration. The average length change of the four bars is expressed as a percentage of the original effective length.

The measurements at 21, 28, and 30 days are a modification to the ASTM procedure requested by the Pittsburgh District. This modification is to ensure potential aggregate expansion is measured, as some local aggregates have historically been slow to react within the standard 14-day testing time frame. 


\section{Results and Discussion}

All mixing and testing presented in this study closely followed the procedures specified in ASTM C1260 and ASTM C1567. The pass/fail limiting criteria for the tests conducted in this report are based on the specifications given in the Unified Facilities Guide Specification (UFGS) Division o3 Concrete Section 0330 00.50 aggregate quality requirement limiting criteria for ASR reactivity. The UFGS states that "final approval of the concrete constituents will be based on the requirements for each individual constituent and that the final proportioned concrete for each mix design meets the reactivity requirement of measured expansion less than 0.08 percent at 32 days after casting (30 days of testing) in accordance with ASTM C1567.

The following summary of results are based on the average expansions of a minimum of three mortar bars per mixture. Detailed test data worksheets of all ASTM C1260 and ASTM C1567 are provided in Appendices B and C, respectively.

\subsection{ASTM C1260}

Results of the ASTM C126o for the three test aggregates (Hanson, Shelly, and Georgetown) tested are provided in Table 6 and illustrated in Figure 1. The length change percent is an average of four mortar bars.

Table 6. Average length change percent of all project aggregates in ASTM C1260.

\begin{tabular}{|c|c|c|c|}
\hline \multirow{2}{*}{ Age, Days } & \multicolumn{3}{|c|}{ Aggregate Source } \\
\cline { 2 - 4 } & Hanson & Georgetown & Shelly \\
\hline 0 & 0 & 0 & 0 \\
\hline 3 & 0.0385 & 0.0175 & 0.0053 \\
\hline 5 & 0.0670 & 0.0485 & 0.0343 \\
\hline 7 & 0.1123 & 0.0770 & 0.0697 \\
\hline 14 & 0.1658 & 0.1505 & 0.1420 \\
\hline 21 & 0.1808 & 0.1663 & 0.1490 \\
\hline 28 & 0.1935 & 0.1665 & 0.1603 \\
\hline 30 & 0.1970 & 0.1683 & 0.1623 \\
\hline
\end{tabular}


Figure 1. Mortar bar expansions of Hanson, Georgetown, and Shelly aggregates in ASTM C1260.

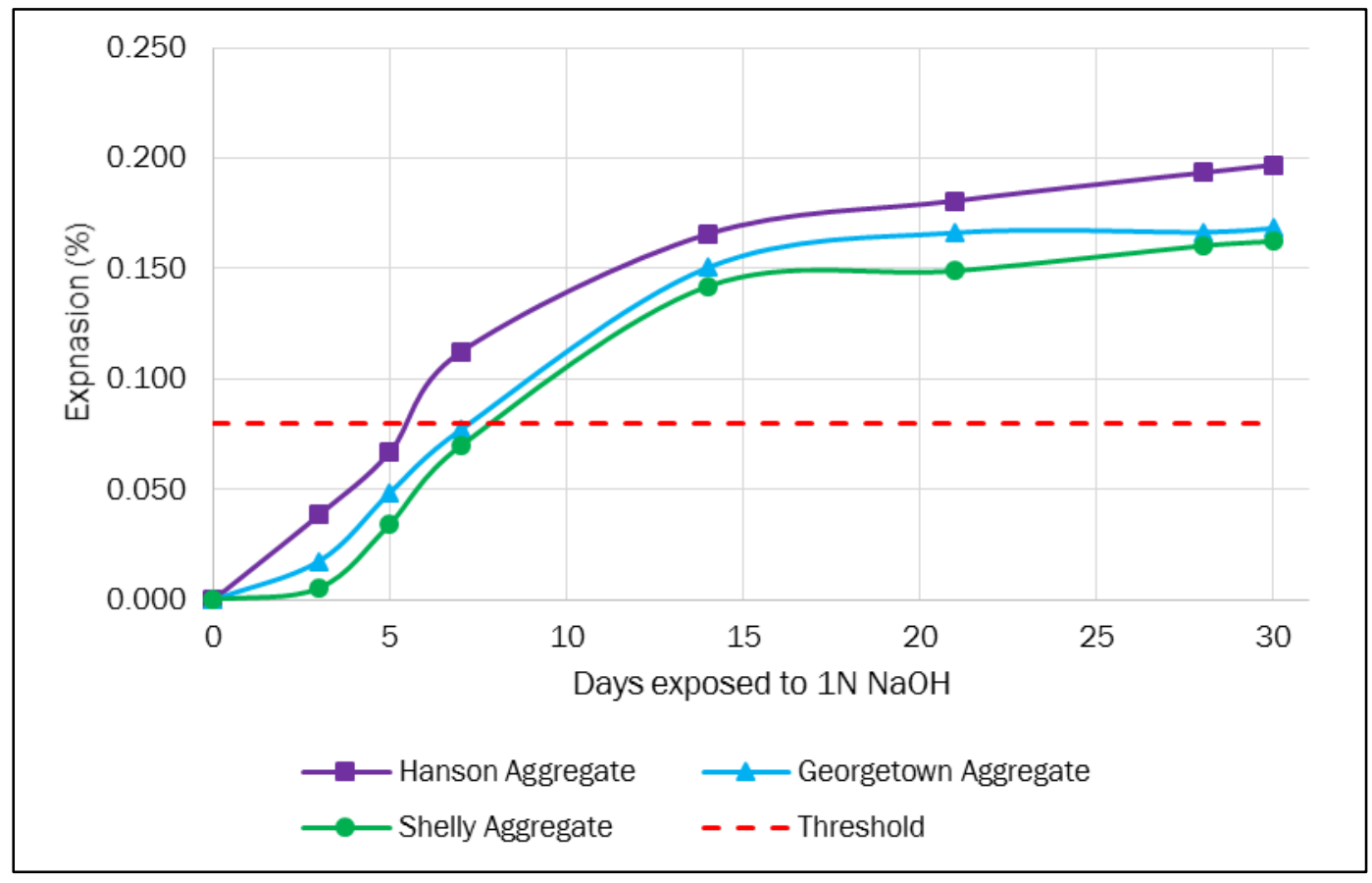

Based on the project limiting criteria, i.e., expansions less than $0.08 \%$ at 30 days, all aggregates are considered potentially reactive with final 30day mortar bar expansions of $0.1970 \%$ for Hanson, $0.1683 \%$ for Georgetown, and $0.1623 \%$ for Shelly aggregates.

\subsection{ASTM C1567}

Results of the ASTM C1567 test are presented in Table 7. The length change percent given is an average of four mortar bars. The average expansion at 30 days of all 48 different test mixtures fell below the $0.08 \%$ limiting criteria.

It is notable to mention that although still passing, the Hanson source aggregate resulted in higher expansions compared to the Shelly and Georgetown source aggregates. This compares with the ASTM C1260 results of the Hanson aggregate producing the highest expansions. This is possibly explained by the Hanson source being a coarse aggregate and the Shelly and Georgetown sources being fine aggregates. More preparations were required for the coarse aggregate to meet the graduation requirements, and the crushing of the material could affect the expansion. 
Table 7. Results of all ASTM C1567 tests at 30 days.

\begin{tabular}{|c|c|c|c|}
\hline Mixture ID & Aggregate Source & Fly Ash Source & Average \% Expansion \\
\hline \multirow{12}{*}{ Mix 1} & \multirow{4}{*}{ Hanson } & Sammis & 0.0183 \\
\hline & & Ft. Martin & 0.0198 \\
\hline & & Longview & 0.0200 \\
\hline & & Brandon Shores & 0.0185 \\
\hline & \multirow{4}{*}{ Georgetown } & Sammis & 0.0075 \\
\hline & & Ft. Martin & 0.0075 \\
\hline & & Longview & 0.0093 \\
\hline & & Brandon Shores & 0.0090 \\
\hline & \multirow{4}{*}{ Shelly } & Sammis & 0.0090 \\
\hline & & Ft. Martin & 0.0093 \\
\hline & & Longview & 0.0073 \\
\hline & & Brandon Shores & 0.0075 \\
\hline \multirow{12}{*}{ Mix 5} & \multirow{4}{*}{ Hanson } & Sammis & 0.0443 \\
\hline & & Ft. Martin & 0.0470 \\
\hline & & Longview & 0.0595 \\
\hline & & Brandon Shores & 0.0440 \\
\hline & \multirow{4}{*}{ Georgetown } & Sammis & 0.0185 \\
\hline & & Ft. Martin & 0.0170 \\
\hline & & Longview & 0.0188 \\
\hline & & Brandon Shores & 0.0198 \\
\hline & \multirow{4}{*}{ Shelly } & Sammis & 0.0160 \\
\hline & & Ft. Martin & 0.0147 \\
\hline & & Longview & 0.0208 \\
\hline & & Brandon Shores & 0.0193 \\
\hline \multirow{12}{*}{ Mix 7} & \multirow{4}{*}{ Hanson } & Sammis & 0.0333 \\
\hline & & Ft. Martin & 0.0333 \\
\hline & & Longview & 0.0350 \\
\hline & & Brandon Shores & 0.0337 \\
\hline & \multirow{4}{*}{ Georgetown } & Sammis & 0.0130 \\
\hline & & Ft. Martin & 0.0168 \\
\hline & & Longview & 0.0213 \\
\hline & & Brandon Shores & 0.0195 \\
\hline & \multirow{4}{*}{ Shelly } & Sammis & 0.0130 \\
\hline & & Ft. Martin & 0.0160 \\
\hline & & Longview & 0.0223 \\
\hline & & Brandon Shores & 0.0200 \\
\hline \multirow{12}{*}{ Mix 10} & \multirow{4}{*}{ Hanson } & Sammis & 0.0638 \\
\hline & & Ft. Martin & 0.0593 \\
\hline & & Longview & 0.0728 \\
\hline & & Brandon Shores & 0.0593 \\
\hline & \multirow{4}{*}{ Georgetown } & Sammis & 0.0123 \\
\hline & & Ft. Martin & 0.0130 \\
\hline & & Longview & 0.0188 \\
\hline & & Brandon Shores & 0.0148 \\
\hline & \multirow{4}{*}{ Shelly } & Sammis & 0.0135 \\
\hline & & Ft. Martin & 0.0138 \\
\hline & & Longview & 0.0167 \\
\hline & & Brandon Shores & 0.0150 \\
\hline
\end{tabular}




\subsubsection{Mix 1}

The average length change data are shown in Table 8 for all 12 combinations of materials using Mix 1. Figure 2 compares the three aggregates tested against the four fly ash sources for the Mix 1 design. The Hanson aggregate is clearly the most reactive aggregate in Mix 1 regardless of the fly ash used. The graphs illustrate similar expansions in both the Shelly and Georgetown aggregates. The largest expansion at 30 days was $0.02 \%$ from the Hanson aggregate and Longview fly ash combination. The smallest expansion at 30 days, i.e., 0.0073\%, was from the Shelly aggregate and Longview fly ash combination. All 12 combinations of Mix 1 meet the project requirement of expansions less than $0.08 \%$ at 30 days.

Table 8. Average length change of mortar bars made from Mix 1 materials.

\begin{tabular}{|c|c|c|c|c|c|c|c|c|c|c|c|c|}
\hline \multirow{2}{*}{ Days } & \multicolumn{3}{|c|}{ Sammis Fly Ash } & \multicolumn{3}{c|}{ Ft. Martin Fly Ash } & \multicolumn{3}{c|}{ Longview Fly Ash } & \multicolumn{3}{c|}{ Brandon Shores Fly Ash } \\
\cline { 2 - 11 } & $* H$ & $* G$ & $* S$ & $* H$ & $* G$ & $* S$ & $*_{H}$ & $*_{G}$ & $*_{S}$ & $* H$ & $*_{G}$ & $* S$ \\
\hline 0 & 0.0000 & 0.0000 & 0.0000 & 0.0000 & 0.0000 & 0.0000 & 0.0000 & 0.0000 & 0.0000 & 0.0000 & 0.0000 & 0.0000 \\
\hline 5 & 0.0053 & 0.0020 & 0.0017 & 0.0060 & 0.0008 & 0.0028 & 0.0047 & 0.0023 & 0.0013 & 0.0038 & 0.0023 & 0.0013 \\
\hline 7 & 0.0060 & 0.0038 & 0.0040 & 0.0110 & 0.0025 & 0.0035 & 0.0073 & 0.0040 & 0.0023 & 0.0090 & 0.0040 & 0.0040 \\
\hline 14 & 0.0100 & 0.0048 & 0.0057 & 0.0118 & 0.0040 & 0.0055 & 0.0107 & 0.0060 & 0.0043 & 0.0118 & 0.0063 & 0.0055 \\
\hline 21 & 0.0157 & 0.0050 & 0.0073 & 0.0143 & 0.0060 & 0.0070 & 0.0170 & 0.0073 & 0.0047 & - & - & - \\
\hline 28 & 0.0167 & 0.0075 & 0.0083 & 0.0180 & 0.0070 & 0.0085 & 0.0183 & 0.0083 & 0.0063 & 0.0160 & 0.0088 & 0.0075 \\
\hline 30 & 0.0183 & 0.0075 & 0.0090 & 0.0198 & 0.0075 & 0.0093 & 0.0200 & 0.0093 & 0.0073 & 0.0185 & 0.0090 & 0.0075 \\
\hline
\end{tabular}

*Notes:

$\mathrm{H}$ - Hanson Aggregate

G- Georgetown Aggregate

S-Shelly Aggregate 
Figure 2. Mortar bar expansions versus time for Mix 1.

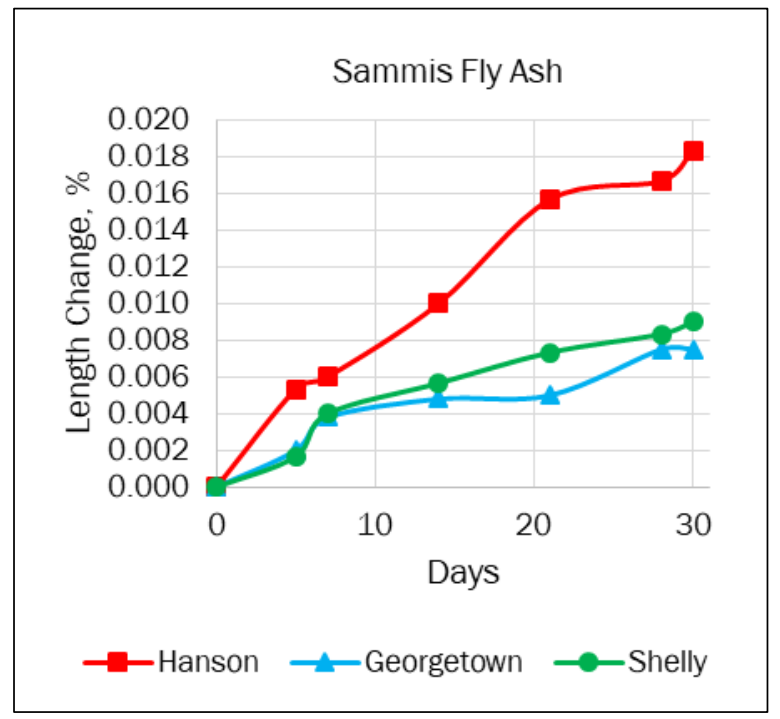

a) Sammis Fly Ash.

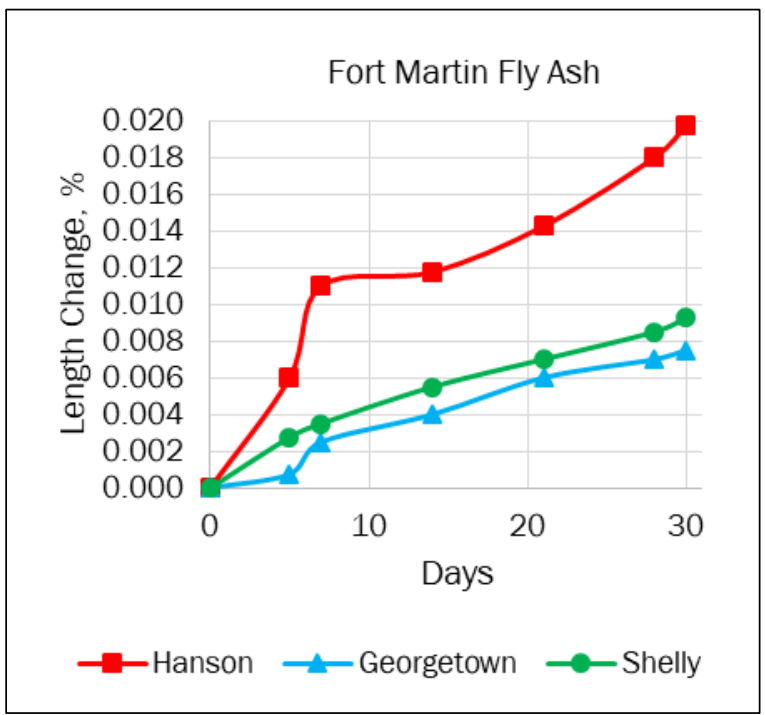

b) Ft. Martin Fly Ash.

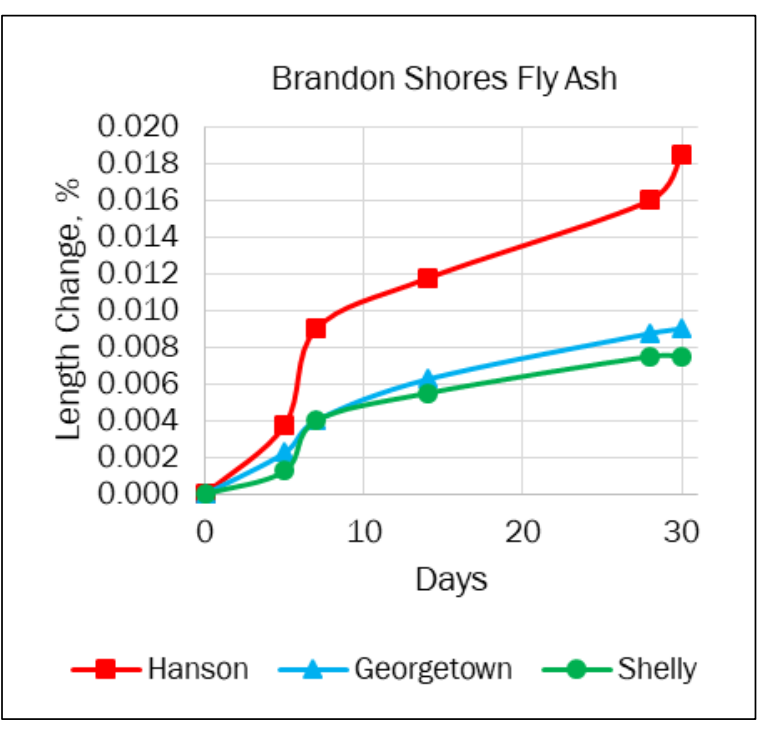

c) Brandon Shores Fly Ash.

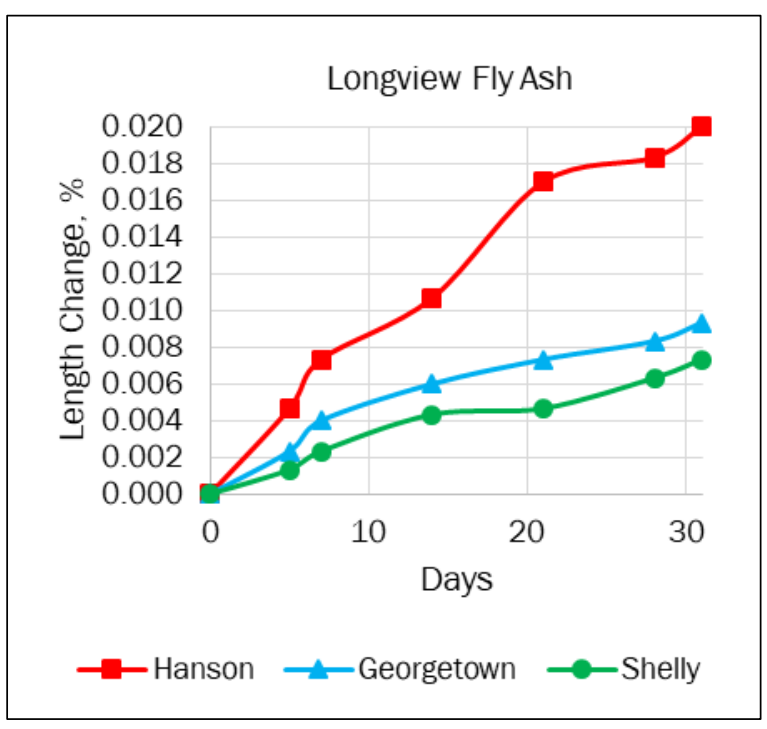

d) Longview Fly Ash. 


\subsubsection{Mix 5}

The average length change data are shown in Table 9 for all 12 combinations of materials using the Mix 5 design. Figure 3 compares the three aggregates tested against the four fly ash sources. Similar to the trends in Mix 1, the Hanson aggregate is the most reactive aggregate in Mix 5 regardless of the fly ash used. The highest 30-day length change was 0.0595\% from a combination of the Longview fly ash and Hanson aggregate. The lowest 30-day length change was 0.0147\% from a combination of Fort Martin fly ash source and Shelly sand.

Table 9. Average length change of mortar bars made from Mix 5 materials.

\begin{tabular}{|c|c|c|c|c|c|c|c|c|c|c|c|c|}
\hline \multirow{2}{*}{ Days } & \multicolumn{3}{|c|}{ Sammis Fly Ash } & \multicolumn{3}{c|}{ Ft. Martin Fly Ash } & \multicolumn{3}{c|}{ Longview Fly Ash } & \multicolumn{3}{c|}{ Brandon Shores Fly Ash } \\
\cline { 2 - 12 } & $* H$ & $*_{G}$ & $* S$ & $* H$ & $*_{G}$ & $*_{S}$ & $*_{H}$ & $*_{G}$ & $*_{S}$ & $*_{H}$ & $*_{G}$ & $*_{S}$ \\
\hline 0 & 0.0000 & 0.0000 & 0.0000 & 0.0000 & 0.0000 & 0.0000 & 0.0000 & 0.0000 & 0.0000 & 0.0000 & 0.0000 & 0.0000 \\
\hline 3 & - & 0.0000 & - & - & - & - & 0.0043 & -.0013 & 0.0038 & 0.0027 & 0.0043 & 0.0018 \\
\hline 5 & 0.0038 & 0.0050 & 0.0040 & 0.0030 & 0.0030 & 0.0063 & - & - & - & 0.0057 & 0.0070 & 0.0080 \\
\hline 7 & 0.0080 & 0.0053 & 0.0065 & 0.0073 & 0.0053 & 0.0080 & 0.0123 & 0.0008 & 0.0035 & 0.0117 & 0.0078 & 0.0070 \\
\hline 14 & 0.0173 & 0.0085 & 0.0090 & 0.0158 & 0.0067 & 0.0100 & 0.0170 & 0.0035 & 0.0025 & 0.0330 & 0.0125 & 0.0120 \\
\hline 21 & 0.0278 & 0.0123 & 0.0113 & 0.0268 & 0.0093 & 0.0100 & 0.0358 & 0.0123 & 0.0105 & - & - & - \\
\hline 28 & 0.0420 & 0.0175 & 0.0138 & 0.0465 & 0.0150 & 0.0137 & 0.0595 & 0.0173 & 0.0178 & 0.0427 & 0.0178 & 0.0170 \\
\hline 30 & 0.0443 & 0.0185 & 0.016 & 0.0470 & 0.0170 & 0.0147 & 0.0595 & 0.0188 & 0.0208 & 0.0440 & 0.0198 & 0.0193 \\
\hline
\end{tabular}

*Notes:

$\mathrm{H}$ - Hanson Aggregate

G - Georgetown Aggregate

$S$ - Shelly Aggregate 
Figure 3. Mortar bar expansions versus time for Mix 5.

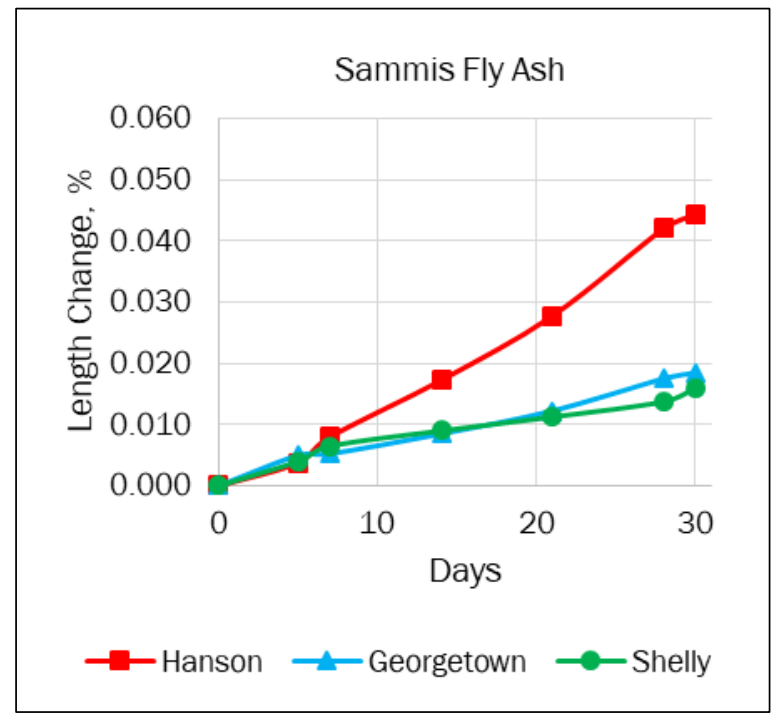

a) Sammis Fly Ash.

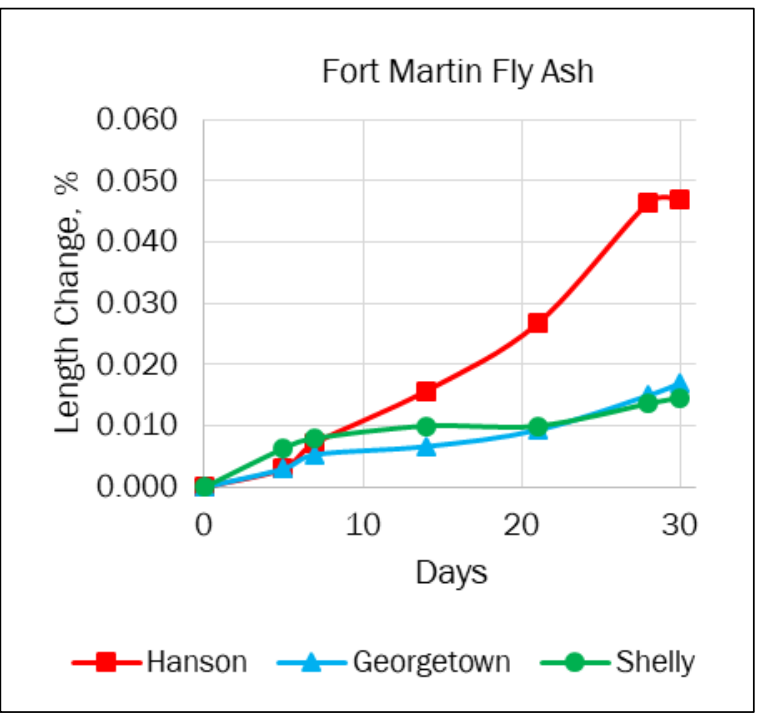

b) Ft. Martin Fly Ash.

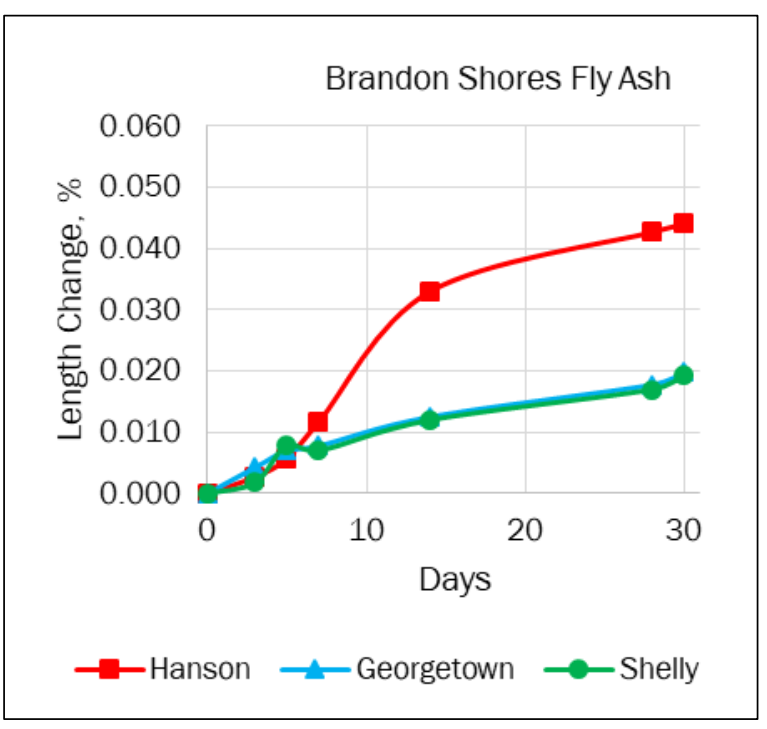

c) Brandon Shores Fly Ash.

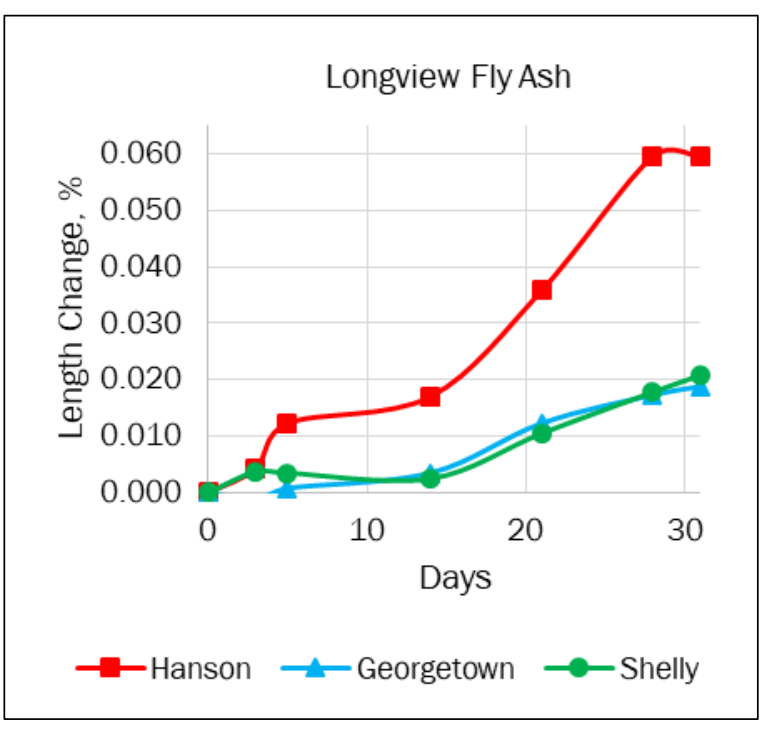

d) Longview Fly Ash. 


\subsubsection{Mix 7}

The average length change data are shown in Table 10 for all 12 combinations of materials using the Mix 7 design. Figure 4 compares the three aggregates tested against the four fly ash sources. Once again, the Hanson aggregate is the most reactive aggregate in Mix 7 regardless of the fly ash used. The highest 30-day length change was $0.0350 \%$ from a combination of the Longview fly ash and Hanson aggregate. The lowest 30-day length change was 0.0130\% in two sources, i.e., the Georgetown aggregate and Sammis fly ash combination and the Shelly aggregate and Sammis fly ash combination.

Table 10. Average length change of mortar bars made from Mix 7 materials.

\begin{tabular}{|c|c|c|c|c|c|c|c|c|c|c|c|c|}
\hline \multirow{2}{*}{ Days } & \multicolumn{3}{|c|}{ Sammis Fly Ash } & \multicolumn{3}{c|}{ Ft. Martin Fly Ash } & \multicolumn{3}{c|}{ Longview Fly Ash } & \multicolumn{3}{c|}{ Brandon Shores Fly Ash } \\
\cline { 2 - 11 } & $* H$ & $* G$ & $* S$ & $* H$ & $* G$ & $* S$ & $*_{H}$ & $*_{G}$ & $* S$ & $* H$ & $*_{G}$ & $* S$ \\
\hline 0 & 0.0000 & 0.0000 & 0.0000 & 0.0000 & 0.0000 & 0.0000 & 0.0000 & 0.0000 & 0.0000 & 0.0000 & 0.0000 & 0.0000 \\
\hline 3 & 0.0043 & 0.0035 & 0.0033 & 0.0015 & 0.0025 & 0.0023 & 0.0013 & 0.0033 & 0.0035 & 0.0027 & 0.0013 & 0.0005 \\
\hline 5 & 0.0108 & 0.0050 & 0.0073 & 0.0083 & 0.0075 & 0.0053 & - & - & - & 0.0057 & 0.0043 & 0.0048 \\
\hline 7 & 0.0145 & 0.0075 & 0.0093 & 0.0150 & 0.0095 & 0.0088 & 0.0037 & 0.0033 & 0.0050 & 0.0100 & 0.0045 & 0.0085 \\
\hline 14 & 0.0235 & 0.0098 & 0.0107 & 0.0213 & 0.0128 & 0.0103 & 0.0137 & 0.0047 & 0.0063 & 0.0190 & 0.0098 & 0.0135 \\
\hline 21 & - & - & - & - & - & - & - & 0.0100 & 0.0165 & - & - & - \\
\hline 28 & 0.0313 & 0.0115 & 0.0120 & 0.0298 & 0.0148 & 0.0133 & 0.0380 & 0.0170 & 0.0188 & 0.0327 & 0.0173 & 0.0183 \\
\hline 30 & 0.0333 & 0.0130 & 0.0130 & 0.0333 & 0.0168 & 0.0160 & 0.0350 & 0.0213 & 0.0223 & 0.0337 & 0.0195 & 0.0200 \\
\hline
\end{tabular}

*Notes:

$\mathrm{H}$ - Hanson Aggregate

G - Georgetown Aggregate

$S$ - Shelly Aggregate 
Figure 4. Mortar bar expansions versus time for Mix 7.

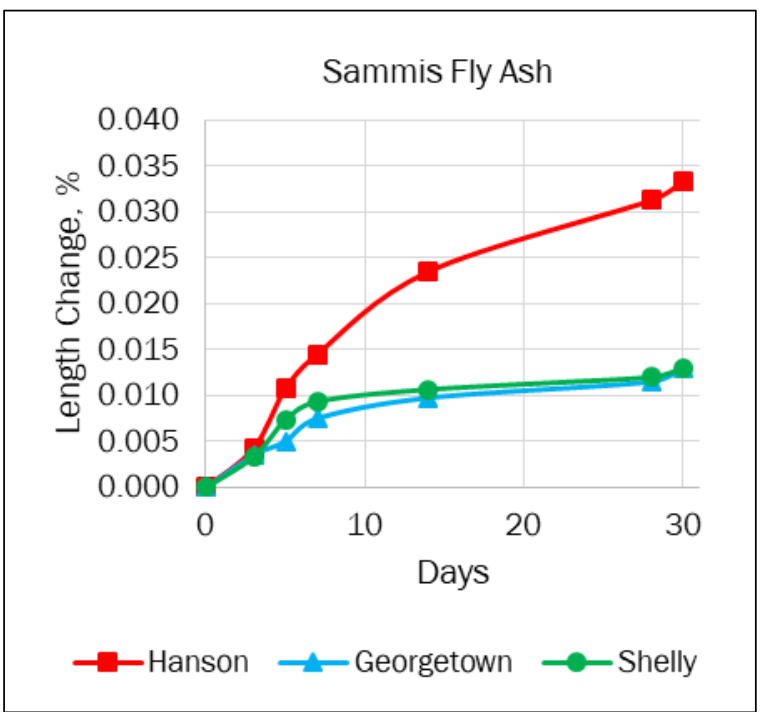

a) Sammis Fly Ash.

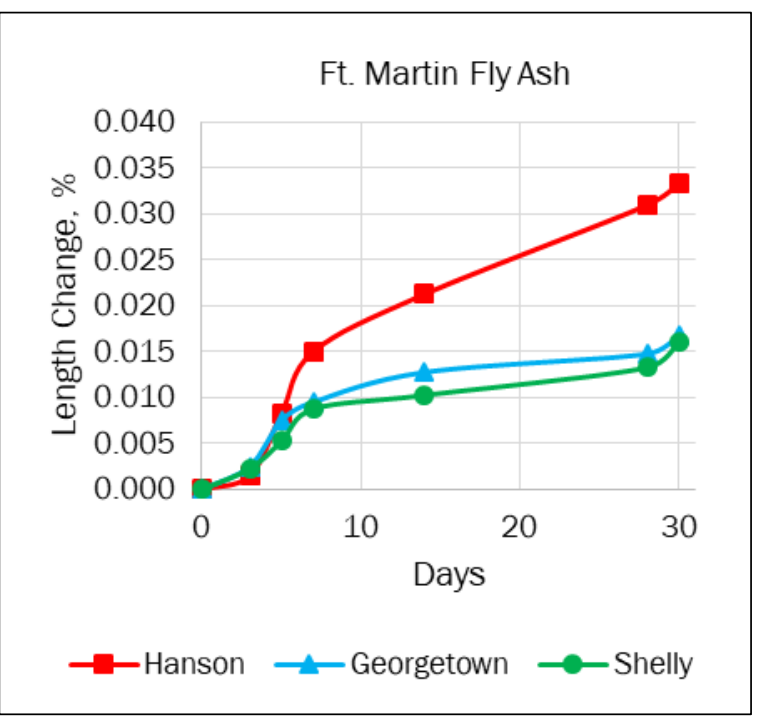

b) Ft. Martin Fly Ash.

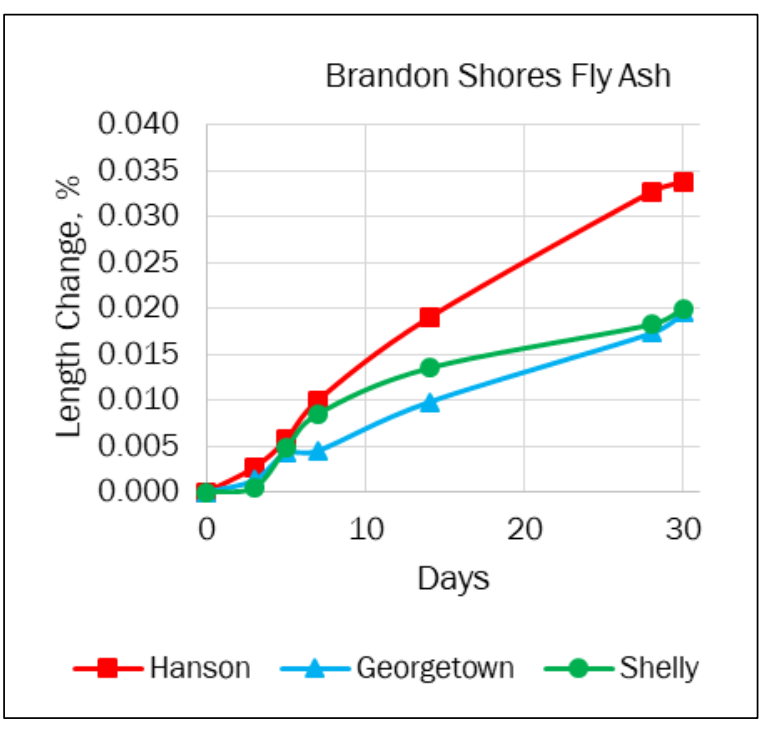

c) Brandon Shores Fly Ash.

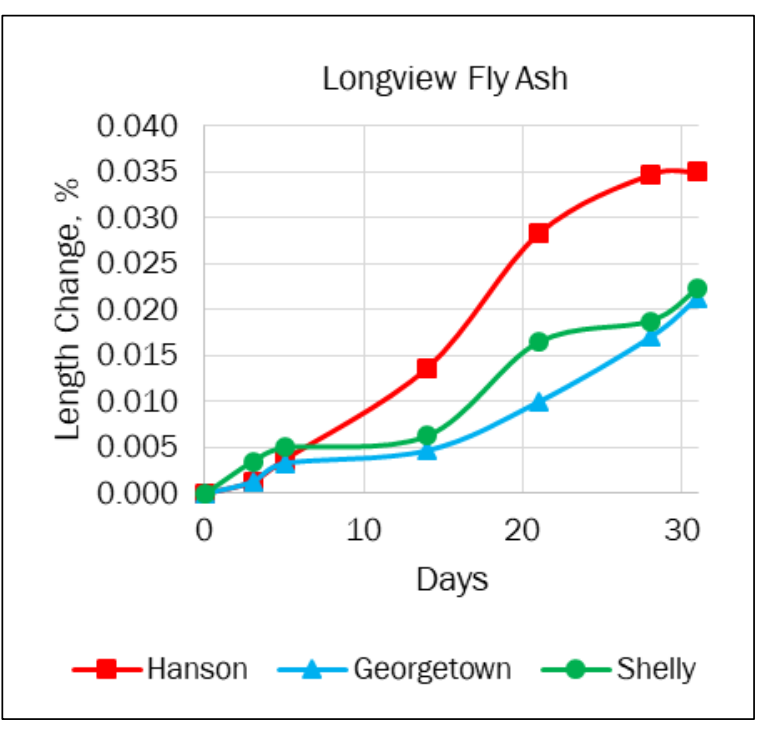

d) Longview Fly Ash. 


\subsubsection{Mix 10}

The average length change data are shown in Table 11 for all 12 combinations of materials using the Mix 10 design. Figure 5 compares the three aggregates tested against the four fly ash sources. The Hanson aggregate is the most reactive aggregate in Mix 10 regardless of the fly ash used, which is consistent with the other three mix designs. The highest 30day length change was $0.0728 \%$ from a combination of the Longview fly ash and Hanson aggregate. The lowest 30-day length change was $0.0123 \%$ from a combination of the Georgetown aggregate and Sammis fly ash source.

Table 11. Average length change of mortar bars made from Mix 10 materials.

\begin{tabular}{|c|c|c|c|c|c|c|c|c|c|c|c|c|}
\hline \multirow{2}{*}{ Days } & \multicolumn{3}{|c|}{ Sammis Fly Ash } & \multicolumn{3}{c|}{ Ft. Martin Fly Ash } & \multicolumn{3}{c|}{ Longview Fly Ash } & \multicolumn{3}{c|}{ Brandon Shores Fly Ash } \\
\cline { 2 - 12 } & $* H$ & $* G$ & $* S$ & $* H$ & $* G$ & $* S$ & $* H$ & $* G$ & $* S$ & $* H$ & $* G$ & $* S$ \\
\hline 0 & 0.0000 & 0.0000 & 0.0000 & 0.0000 & 0.0000 & 0.0000 & 0.0000 & 0.0000 & 0.0000 & 0.0000 & 0.0000 & 0.0000 \\
\hline 3 & - & 0.0047 & - & - & - & - & 0.0060 & 0.0003 & 0.0007 & 0.0028 & 0.0025 & 0.0010 \\
\hline 5 & 0.0120 & 0.0037 & 0.0003 & 0.0097 & 0.0043 & 0.0025 & - & - & - & 0.0053 & 0.0028 & 0.0023 \\
\hline 7 & 0.0138 & 0.0067 & 0.0033 & 0.0123 & 0.0053 & 0.0062 & 0.0113 & 0.0013 & 0.0033 & 0.0133 & 0.0033 & 0.0030 \\
\hline 14 & 0.0300 & 0.0100 & 0.0088 & 0.0267 & 0.0088 & 0.0070 & 0.0290 & 0.0045 & 0.0043 & 0.0273 & 0.0070 & 0.0093 \\
\hline 21 & 0.0540 & 0.0147 & 0.0105 & 0.0397 & 0.0088 & 0.0105 & 0.0408 & 0.0098 & 0.0070 & - & - & - \\
\hline 28 & 0.0605 & 0.0147 & 0.0123 & 0.0563 & 0.0100 & 0.0120 & 0.0665 & 0.0165 & 0.0130 & 0.0565 & 0.0135 & 0.0143 \\
\hline 30 & 0.0638 & 0.0163 & 0.0135 & 0.0593 & 0.0130 & 0.0138 & 0.0728 & 0.0188 & 0.0167 & 0.0593 & 0.0148 & 0.0150 \\
\hline
\end{tabular}

*Notes:

$\mathrm{H}$ - Hanson Aggregate

G - Georgetown Aggregate

$S$ - Shelly Aggregate 
Figure 5. Mortar bar expansions versus time for Mix 10.

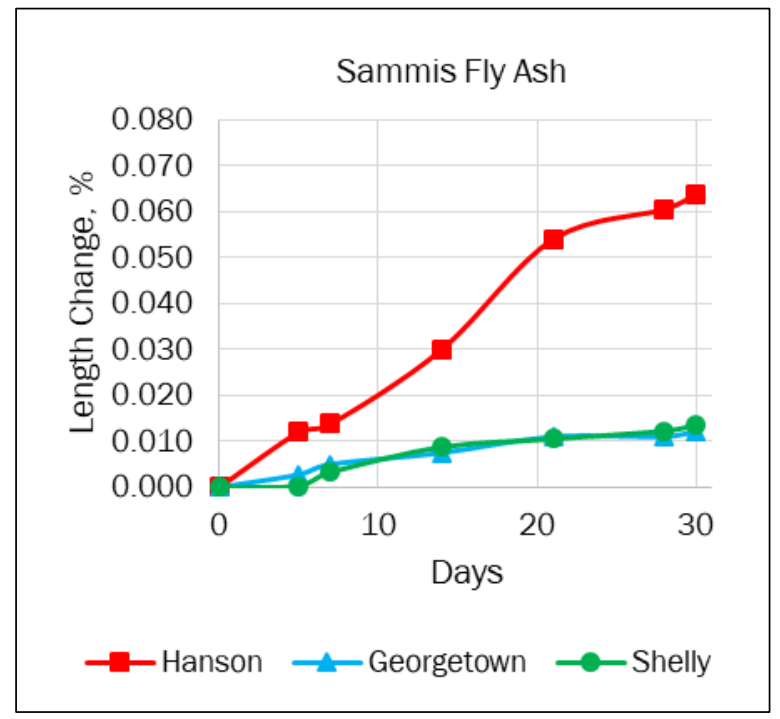

a) Sammis Fly Ash.

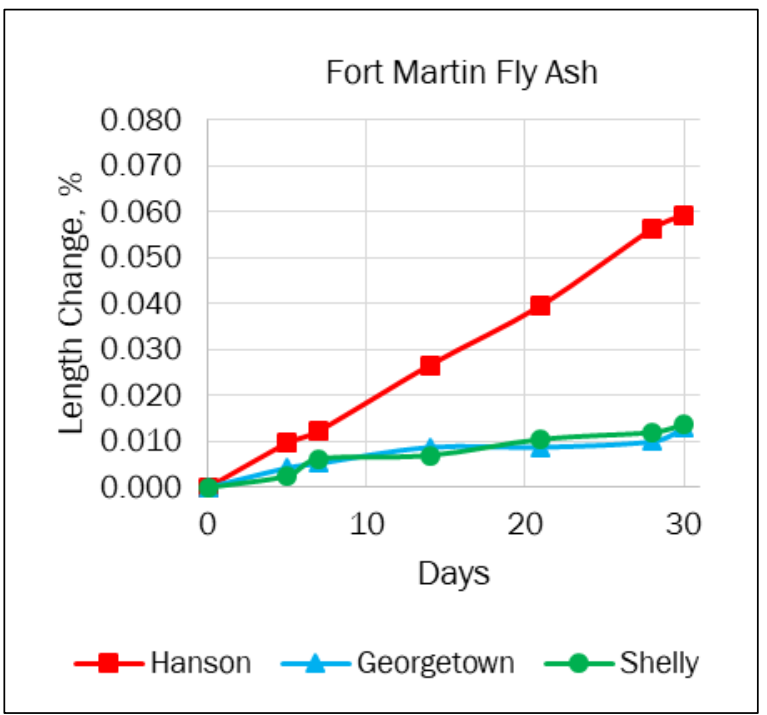

b) Ft. Martin Fly Ash.

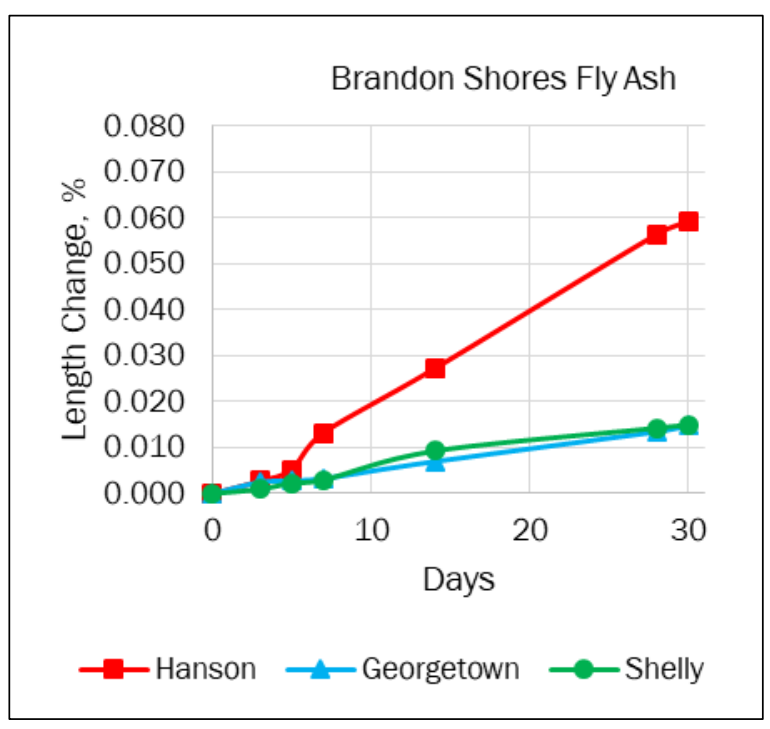

c) Brandon Shores Fly Ash.

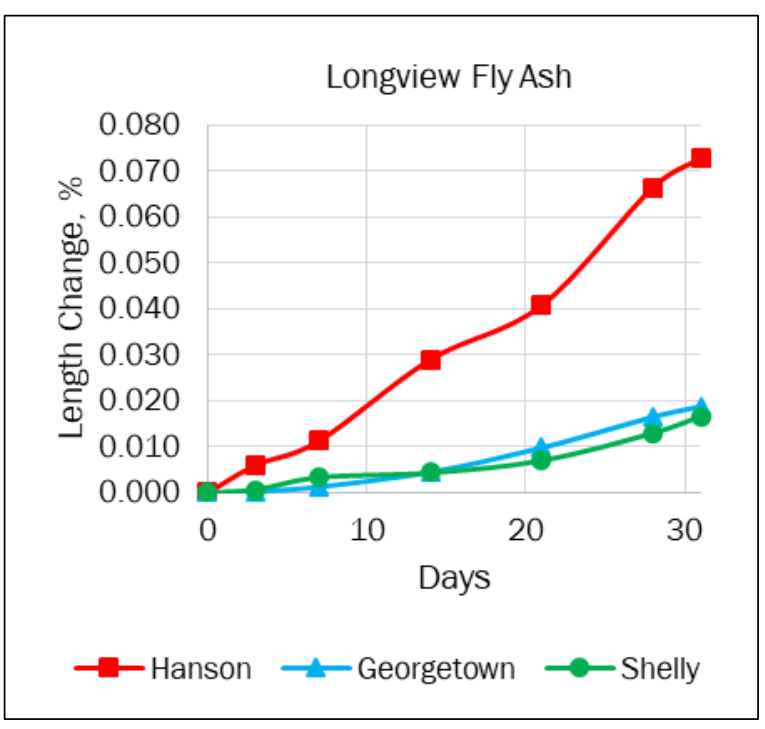

d) Longview Fly Ash. 


\section{Summary and Conclusions}

This testing investigated the potential ASR reactivity of 3 aggregate sources in accordance to ASTM C1260 and the efficacy to mitigate expansion due to potential ASR using a combination of cementitious materials and aggregates in accordance to ASTM C1567. A total of 48 different mixtures were produced from a combination of four fly ash sources (Sammis, Fort Martin, Longview, and Brandon Shores), three aggregate sources (Shelly, Hanson, and Georgetown), and four mix designs (Mix 1, 5, 7, and 10). The following conclusions are made based on the results presented herein.

- All project aggregate sources (Hanson, Georgetown, and Shelly) are considered alkali-silica reactive based on the limiting criteria of the project specification, i.e., expansions less than $0.08 \%$ at 30 days, when tested in accordance to the ASTM C1260.

- The Hanson coarse aggregate source resulted in the highest 30-day expansion of $0.1970 \%$, whereas the Georgetown and Shelly fine aggregate sources produced similar expansions of $0.1683 \%$ and $0.1623 \%$, respectively.

- All 48 combinations of materials and mixture designs resulted in 30day expansions less $0.08 \%$ when tested in accordance to the ASTM C1567. These results indicate that the constituent project materials in the proposed mix designs can effectively mitigate ASR.

- The Hanson aggregate/Longview fly ash combination resulted in the largest expansions of all four mix design groups. Final 30-day expansions with this aggregate/fly ash combination were $0.02 \%$ in Mix 1, 0.0595\% in Mix 5, 0.035\% in Mix 7, and 0.0728\% in Mix 10.

- The Georgetown aggregate/Sammis fly ash combination resulted in the lowest expansions in Mix 1, Mix 7, and Mix 10. Final 30-day expansions with this aggregate/fly ash combination were $0.0073 \%$ in Mix 1, $0.013 \%$ in Mix 7, and 0.0123\% in Mix 10. The highest lowest expansion in Mix 5 was $0.0147 \%$ from the Shelly aggregate/Fort Martin fly ash combination.

- Higher expansions in the Hanson coarse aggregate compared with the Shelly and Georgetown fine aggregates is possibly due to the additional crushing involved in the coarse aggregate source to the gradation sizes required for both the ASTM C1260 and ASTM C1567 testing. 


\section{Appendix A: Material Reports}

\section{A.1 Cementitious materials}

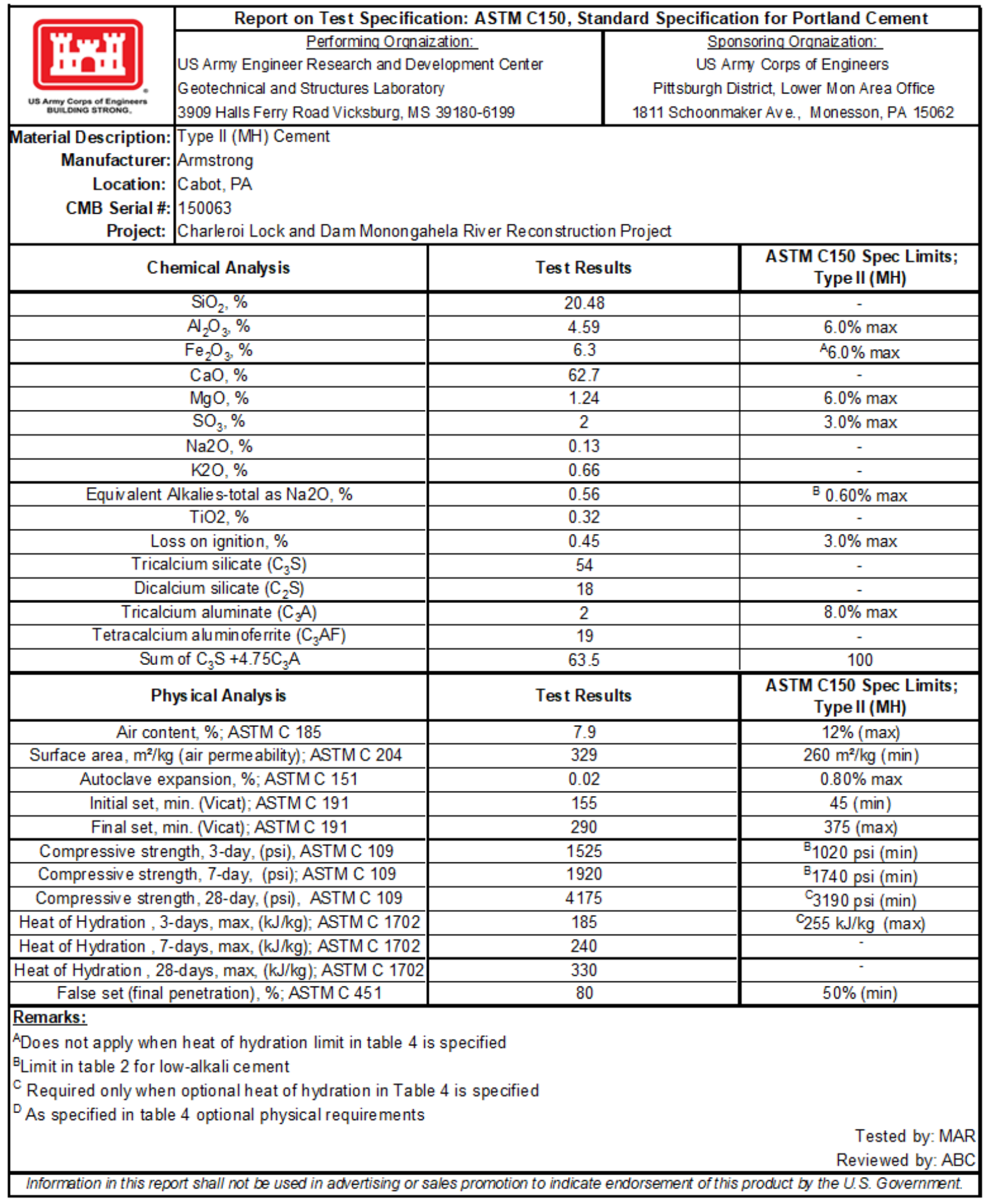




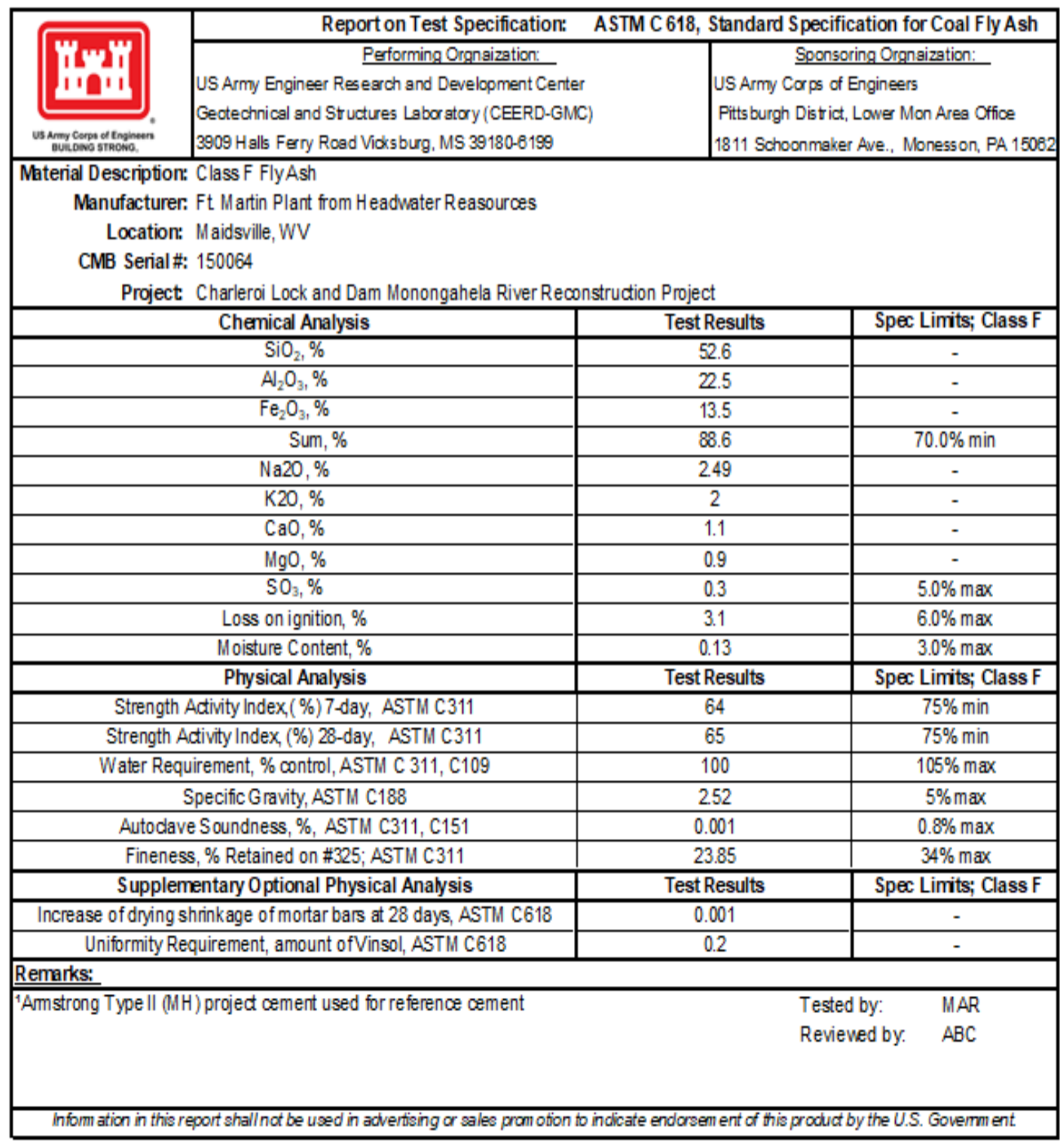




\begin{tabular}{|c|c|c|c|c|}
\hline & Report on Test Specification: & \multicolumn{3}{|c|}{ ASTM C 618, Standard Specification for Coal Fly Ash } \\
\hline 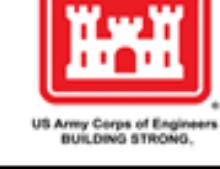 & $\begin{array}{l}\text { Performing Orgnaization: } \\
\text { US Army Engineer Research and Development Cen } \\
\text { Geotechnical and Structures Laboratory (CEERD-G } \\
3909 \text { Halls Ferry Road Vidksburg, MS 39180-6199 }\end{array}$ & $\begin{array}{l}\text { US Arm } \\
\text { Pitts bur } \\
1811 \mathrm{So}\end{array}$ & \multicolumn{2}{|c|}{$\begin{array}{l}\text { Pitts burgh District, Lower Mon Area Office } \\
1811 \text { Schœonmeker Ave., Moness on, PA } 15062\end{array}$} \\
\hline $\begin{array}{r}\text { Material Descript } \\
\text { Manufactu } \\
\text { Locati } \\
\text { CMB Serie } \\
\text { Proje }\end{array}$ & $\begin{array}{l}\text { Class F Fly Ash } \\
\text { W.H. Sammis Plant, Headwaters Reasources } \\
\text { Stratton, OH } \\
150065 \\
\text { Charleroi Lodk and Dam Monongahela River Re }\end{array}$ & nstruction Projed & & \\
\hline \multicolumn{5}{|c|}{ 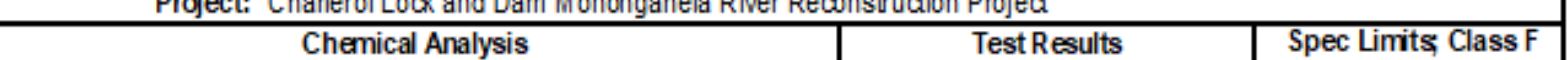 } \\
\hline & $\mathrm{SiO}_{2}, \%$ & 442 & & - \\
\hline & $\mathrm{Al}_{2} \mathrm{O}_{3}, \%$ & 20.2 & & - \\
\hline & $\mathrm{Fe}_{2} \mathrm{O}_{3}, \%$ & 242 & & - \\
\hline & Sum, $\%$ & 88.6 & & $70.0 \% \min$ \\
\hline & $\mathrm{Na} 20, \%$ & 2.87 & & - \\
\hline & $\mathrm{K} 20, \%$ & 2.05 & & - \\
\hline & $\mathrm{CaO}, \%$ & 0 & & - \\
\hline & $\mathrm{MgO}, \%$ & 0.9 & & - \\
\hline & $\mathrm{SO}_{3}, \%$ & 0.6 & & $5.0 \% \max$ \\
\hline & Loss on ignition, $\%$ & 2.99 & & $6.0 \% \max$ \\
\hline & Moisture Content, \% & 0.11 & & $3.0 \% \max$ \\
\hline & Physical Analysis & Test Results & Spec & c Limits Class F \\
\hline Streng & Adivity Index, \%) 7-day, ASTIM C311 & 71 & & $75 \% \min$ \\
\hline Strengt & divity Index, (\%) 28-day, ASTIM C311 & 72 & & $75 \% \mathrm{~min}$ \\
\hline Water $\mathrm{R}$ & jirement, \% control, ASTM C 311, C109 & 100 & & $105 \% \max$ \\
\hline & Specific Gravity, ASTM C188 & 2.6 & & $5 \% \max$ \\
\hline Autoc & e Soundness, \%, ASTM C311, C151 & 0.001 & & $0.8 \% \max$ \\
\hline Fine & s, \% Retained on \#325; ASTM C 311 & 25.92 & & $34 \% \max$ \\
\hline Supp & nentary 0 ptional Physical Analysis & Test Results & Spec & c Limits Class F \\
\hline Increase of dryir & hrinkage of mortar bars at 28 days, ASTM C618 & 0.05 & & - \\
\hline Uniformity & quirement, amount ofVinsol, ASTM C618 & 0.2 & & - \\
\hline \multicolumn{5}{|l|}{ Remarks: } \\
\hline \multicolumn{3}{|c|}{${ }^{2}$ Armstrong Type II (MH) project cement used for reference cement } & \multicolumn{2}{|c|}{$\begin{array}{ll}\text { Tested by. } & \text { MAR } \\
\text { Reviewed by. } & \text { ABC }\end{array}$} \\
\hline
\end{tabular}




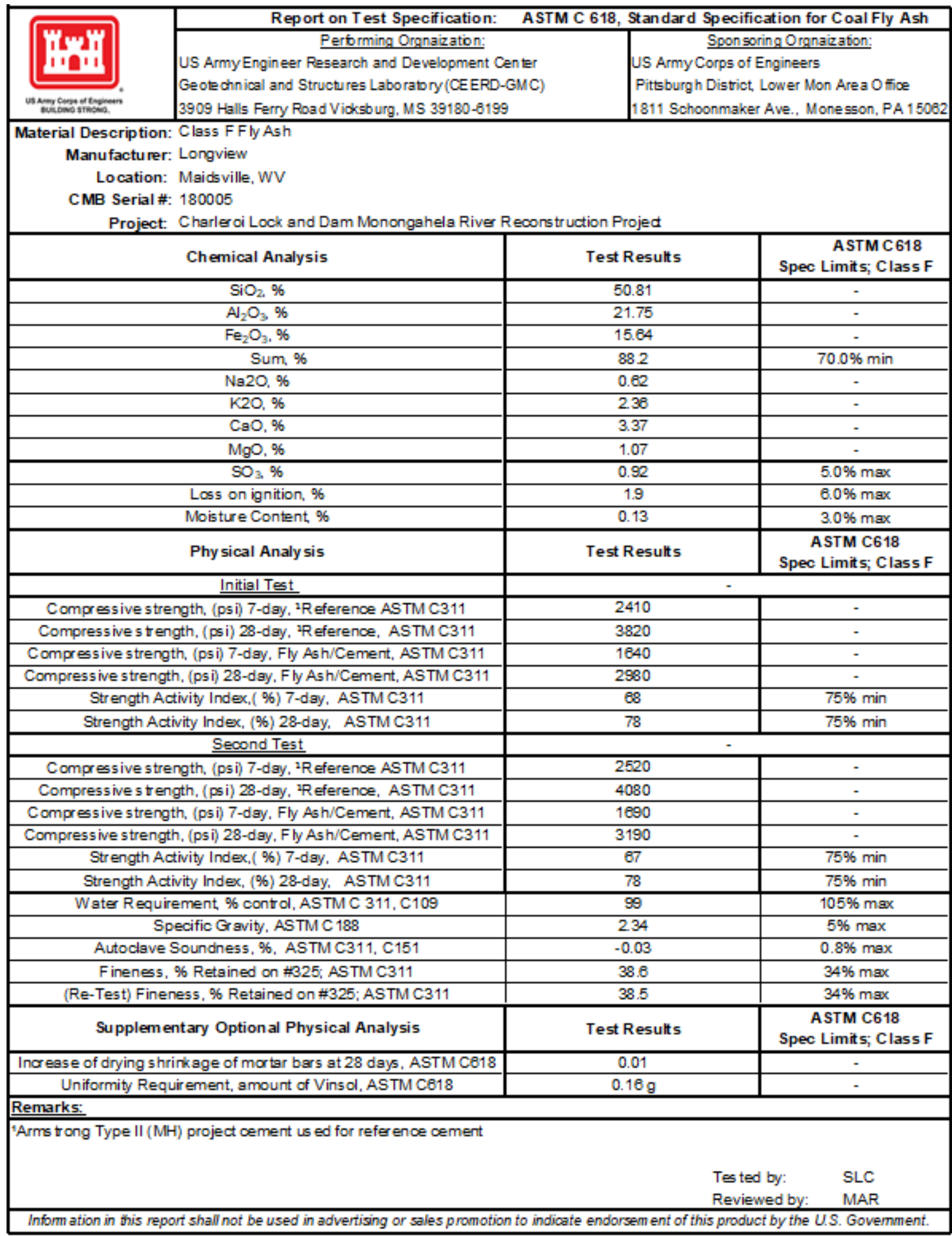




\begin{tabular}{|c|c|c|c|}
\hline & Report on Test Specification: & \multicolumn{2}{|c|}{ ASTM C 618, Standard Specification for Coal Fly Ash } \\
\hline 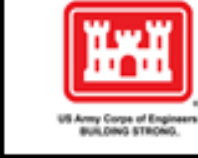 & $\begin{array}{l}\text { Performing Orgnaization: } \\
\text { US Army Engineer Research and Development Center } \\
\text { Geotechnical and St uctures Laboratory (CEERD-GMC) } \\
3909 \text { Halls Ferry Road Vidks burg, MS } 39180-6199\end{array}$ & \multicolumn{2}{|c|}{$\begin{array}{l}\text { Sittsburgh District, Lower Mon Area Office } \\
1811 \text { Schoonmaker Ave., Moness on, PA } 15082\end{array}$} \\
\hline $\begin{array}{r}\text { Material Descrip } \\
\text { Manufac } \\
\text { Loca } \\
\text { CMB Ser } \\
\text { Pro }\end{array}$ & $\begin{array}{l}\text { Class F Fly Ash } \\
\text { Brandon Shores } \\
\text { Curtis Bay, MD } \\
180018 \\
\text { Charleroi Lodk and Dam Monongahela River Recon }\end{array}$ & struction Project & \\
\hline \multicolumn{2}{|r|}{ Chemical Analysis } & Test Results & Spec Limits; Class F \\
\hline & $\mathrm{SiO}_{2}, \%$ & 45.95 & - \\
\hline & $\mathrm{Al}_{2} \mathrm{O}_{3}, \%$ & 22.42 & - \\
\hline & $\mathrm{Fe}_{2} \mathrm{O}_{3}, \%$ & 21.49 & - \\
\hline & Sum, \% & 89.86 & $70.0 \% \min$ \\
\hline & $\mathrm{Na} 2 \mathrm{O}, \%$ & 0.45 & - \\
\hline & $\mathrm{K} 20, \%$ & 1.85 & - \\
\hline & $\mathrm{CaO}, \%$ & 2.64 & - \\
\hline & $\mathrm{MgO}, \%$ & 0.79 & - \\
\hline & $\mathrm{SO}_{3}, \%$ & 0.82 & $5.0 \% \max$ \\
\hline & Loss on ignition, $\%$ & 2 & $6.0 \% \max$ \\
\hline & Moisture Content, \% & 0.3 & $3.0 \% \max$ \\
\hline & Physical Analysis & Test Results & Spec Limits; Class F \\
\hline Compres & trength, (psi) 7-day, ${ }^{2}$ Reference ASTM C311 & 1785 & - \\
\hline Compressi & rength, (psi) 28-day, ${ }^{2}$ Reference, ASTM C311 & 3130 & - \\
\hline Compressive & ingth, (psi) 7-day, Fly Ash/Cement, ASTM C311 & 2320 & - \\
\hline Compressive & ngth, (psi) 28-day, Fly Ash/Cement, ASTM C311 & 4100 & - \\
\hline Stre & Adtivity Index, (\%) 7-day, ASTM C311 & 77 & $75 \% \mathrm{~m}$ in \\
\hline Strer & Activity Index, (\%) 28-day, ASTM C311 & 76 & $75 \% \mathrm{~m}$ in \\
\hline Wate & uirement, \% control, ASTM C 311, C109 & 98 & $105 \% \max$ \\
\hline & Specific Gravity, ASTM C188 & 2.41 & $5 \% \max$ \\
\hline Aut & ve Soundness, \%, ASTM C311, C151 & -0.06 & $0.8 \% \max$ \\
\hline & ss, \% Retained on \#325; ASTM C311 & 19.12 & $34 \% \max$ \\
\hline Sur & nentary Optional Physical Analysis & Test Results & Spec Limits; Class F \\
\hline Increase ofdr & shrinkage of mortar bars at 28 days, ASTM C 618 & 0.01 & - \\
\hline Uniform & equirement, amount of Vinsol, ASTM C618 & 0.15 & - \\
\hline Remarks: & & & \\
\hline${ }^{2 A}$ Arm strong Type & H) project cement used for reference cement & & $\begin{array}{l}\text { by. } \\
\text { ed by: }\end{array}$ \\
\hline
\end{tabular}




\begin{tabular}{|c|c|c|c|}
\hline \multirow[b]{2}{*}{ 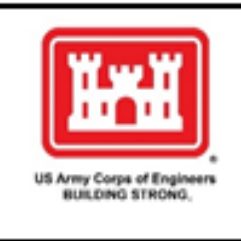 } & \multicolumn{3}{|c|}{ Report on Test Specification: ASTM C 989, Standard Specification for Slag Cement } \\
\hline & $\begin{array}{l}\text { Performing Oranaization: } \\
\text { US Army E ngineer Research and Development Center } \\
\text { Geotechnical and Structures Laboratory (CE ERD-GM C) } \\
3909 \text { Halls Ferry Road Vicksburg, M S 39180-6199 }\end{array}$ & \multicolumn{2}{|c|}{\begin{tabular}{l|l}
\multicolumn{1}{|c|}{ Sponsoring Oran aization: } \\
US Army Corps of En gineers \\
Pittsburgh District, Lo wer Mon Area O ffice \\
1811 Schoonmaker Ave., Monesson, PA 15062
\end{tabular}} \\
\hline $\begin{array}{r}\text { Material Des } \\
\text { Manufact } \\
\text { Locatio } \\
\text { CMB Seri } \\
\text { Projec }\end{array}$ & $\begin{array}{ll}\text { cription: } & \text { Slag Cement, Grade } 100 \\
\text { urer. } & \text { Argos-Essroc } \\
\mathrm{n:} & \text { Middlebranch, OH } \\
\text { al \#: } & 150066 \\
: & \text { Charleroi Lock and Dam M onor }\end{array}$ & ngahela River Reconstr & \\
\hline \multicolumn{2}{|r|}{ Chemical Analysis } & Test Results & Spec Limits \\
\hline \multicolumn{2}{|r|}{ Sulfide Sulfur, S\% } & 2.02 & $2.5 \% \max$ \\
\hline \multicolumn{2}{|r|}{ Sulfate Sulfur, (as SO3) \% } & 0.04 & $4 \% \max$ \\
\hline \multicolumn{2}{|r|}{ Aluminum Oxide, (as Al2O3) \% } & 10.15 & - \\
\hline \multicolumn{2}{|r|}{ Chloride Content of Slag } & 0.05 & - \\
\hline \multicolumn{2}{|r|}{$\mathrm{Na} 2 \mathrm{O}, \%$} & 0.27 & - \\
\hline \multicolumn{2}{|r|}{$\mathrm{K} 2 \mathrm{O}, \%$} & 0.47 & - \\
\hline \multicolumn{2}{|c|}{ Equivalent Alkalies-total as $\mathrm{Na} 2 \mathrm{O}, \%$} & 0.57 & \\
\hline \multicolumn{2}{|r|}{ Physical Analysis } & Test Results & Spec Limits \\
\hline \multicolumn{2}{|c|}{ Compressive strength (psi) 7-day ${ }^{2}$ Reference ASTM C311 } & 1880 & - \\
\hline \multicolumn{2}{|c|}{ Compressive strength (psi) 28-day 'Reference, ASTM C311 } & 3956 & - \\
\hline \multicolumn{2}{|c|}{ Compressive strength (psi) 7-day Slag/Cement, ASTM C311 } & 1583 & - \\
\hline \multicolumn{2}{|c|}{ Compressive strength (psi) 28-day Slag/Cement, ASTM C311 } & 3802 & - \\
\hline \multicolumn{2}{|c|}{ Strength Activity Index, ( \%) 7-day, ASTM C311 } & 88 & $70 \% \min$ \\
\hline \multicolumn{2}{|c|}{ Strength Activity Index, (\%) 28-day, ASTM C311 } & 96 & $90 \% \min$ \\
\hline \multicolumn{2}{|c|}{ Surface area, $\mathrm{m}^{2} / \mathrm{kg}$ (air permeability); ASTM C 204} & 626 & $260 \mathrm{~m}^{2} / \mathrm{kg} \mathrm{min}$ \\
\hline \multicolumn{2}{|r|}{ Specific Gravity, ASTM C188 } & 2.81 & - \\
\hline \multicolumn{2}{|c|}{ Fineness, \% Retained on \#325; ASTM C311 } & 2.8 & $20 \% \max$ \\
\hline \multicolumn{2}{|r|}{ Air content, \%; ASTM C 185} & 4.2 & $12 \% \max$ \\
\hline \multicolumn{4}{|l|}{ Remarks: } \\
\hline \multicolumn{4}{|c|}{${ }^{2}$ Armstrong Type II (MH) project cement used for reference cement } \\
\hline
\end{tabular}




\begin{tabular}{|c|c|c|}
\hline & Report on Test Specification: & AST M C1240, silica Fume U sed in Cementitiou s Mixtures \\
\hline 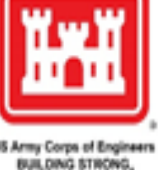 & $\begin{array}{l}\text { Perborming Orgnaization: } \\
\text { US Army Engineer Research and Develo pment Center } \\
\text { Geotechnical and Structures Labora tory (CEERD-GMC) } \\
3909 \mathrm{Halls} \text { Ferry Road Vicksburg, MS } 39180-6199\end{array}$ & $\begin{array}{l}\text { Sponsoring Orgnaization: } \\
\text { US Army Corps of Engineers } \\
\text { Pittsburgh District, Lower Mon Area Ofice } \\
1811 \text { Schoonmaker Ave., Monesson, PA } 15062\end{array}$ \\
\hline
\end{tabular}

Material Description: Microsilica Grade 970 D

Manufacturer: Ekem Materials Inc.

Location: Pitts burgh, PA

CMB Serial \#: 150088

Project: Charleroi Lodk and Dam Monongahela River Reconstruction Project

\begin{tabular}{|c|c|c|}
\hline Chemical Analysis & Test Results & $\begin{array}{l}\text { AST M C1240 } \\
\text { Spec. Limits }\end{array}$ \\
\hline $\mathrm{SiO}_{2}, \%$ & 95.16 & $85 \%$, min. \\
\hline $\mathrm{Al}_{2} \mathrm{O}_{3}, \%$ & 0.24 & - \\
\hline $\mathrm{Fe}_{2} \mathrm{O}_{3}, \%$ & 0.52 & - \\
\hline Sum, $\%$ & 95.92 & - \\
\hline $\mathrm{Na} 2 \mathrm{O}, \%$ & 0.3 & - \\
\hline $\mathrm{K} 2 \mathrm{O}, \%$ & 0.49 & - \\
\hline $\mathrm{MgO}, \%$ & 0.25 & - \\
\hline $\mathrm{SO}_{3}, \%$ & 0.01 & - \\
\hline Loss on ignition, \% & 2.61 & $6 \%, \max$ \\
\hline Moisture \% & 0.61 & $3 \%, \max$ \\
\hline Physical Analysis & Test Results & $\begin{array}{l}\text { AST M C1240 } \\
\text { Spec. Limits }\end{array}$ \\
\hline Control Mixture': Compress ive strength, (psi) 7-day, ASTM C 311 & 1990 & - \\
\hline Test Mixture': Compressive strength, (psi) 7-day, ASTM C 311 & 3280 & $\cdot$ \\
\hline Strength Activity Index, ( \%) 7-day, ASTM C311 & 185 & $105 \%$, min \\
\hline Water Requirement, ASTM C 311,C 109 & 3 & $100-115 \%$ \\
\hline Specific Gravity, ASTM C188 & 2.32 & - \\
\hline Supplimentary Optional Phy sical T ests & Test Results & $\begin{array}{l}\text { AST M C1240 } \\
\text { Spec. Limits }\end{array}$ \\
\hline Uniformity Requirement, amount of Vinsol, ASTM C618 & $0.04 \mathrm{~g}$ & - \\
\hline Effectiveness in Controlling ASR at 14 days, ASTMC 618, C311, C441 & $95 \%$ & $80 \%, \min$. \\
\hline
\end{tabular}

Remarks:

1,2 Armstrong Type II (MH) project cement used for refer ence cement

${ }^{3}$ Following the standard ASTM C 1240 method, $2 \mathrm{~mL}$ of HRWRA required with test mixture to produce flow limits us ing $242 \mathrm{~mL}$ of water

$\begin{array}{ll}\text { Tested by: } & \text { MAR } \\ \text { Reviewed by: } & \text { ABC }\end{array}$




\section{A.2 Aggregate materials}

\begin{tabular}{|c|c|c|c|c|c|c|c|c|c|}
\hline \multicolumn{10}{|c|}{ Summary of Aggregate Tests Report } \\
\hline \multicolumn{3}{|c|}{ Material Description: } & \multicolumn{2}{|c|}{ 3/4-inch limestone aggregate } & \multirow{2}{*}{\multicolumn{2}{|c|}{$\begin{array}{l}\text { Performing } \\
\text { Organizaion: }\end{array}$}} & \multirow{2}{*}{\multicolumn{3}{|c|}{$\begin{array}{l}\text { Geotechnical and Structures Lab } \\
\text { Concrete and Materials Branch }\end{array}$}} \\
\hline & & Source: & \multicolumn{2}{|c|}{ Hanson Aggregates } & & & & & \\
\hline & & Location: & & & \multicolumn{2}{|r|}{ Tested by: } & \multicolumn{3}{|l|}{ R. Hardy } \\
\hline & & CMB Log in \#. & 150070 & & & eviewed by: & J. Burrough & hs/M. Ram & \\
\hline & Sponsorin & g Organization: & USACE Pittsbu & urgh District, Lo & wer Mon Area & a Office & & & \\
\hline & & Project: & Charleroi Lock & and Dam Mono & ngahela River & r Reconstruc & ction Projec & & \\
\hline & & & ASTM C & 136: Sieve Ana & aly sis: & & & & \\
\hline Sign Size & Run & & Cumulative & ve Percent & Run & & Cumulative & e Percent & Avg \% \\
\hline Sleve size & Mass Ret, $\mathrm{g}$ & $\%$ Ret. & Ret. & Pass & Mass Ret, g & \% Ret. & Ret. & Pass & Passing \\
\hline 3 in & 0.0 & $0.00 \%$ & $0.00 \%$ & $100.00 \%$ & 0.0 & $0.00 \%$ & $0.00 \%$ & $100.00 \%$ & $100 \%$ \\
\hline $21 / 2$ in & 0.0 & $0.00 \%$ & $0.00 \%$ & $100.00 \%$ & 0.0 & $0.00 \%$ & $0.00 \%$ & $100.00 \%$ & $100 \%$ \\
\hline 2 in & 0.0 & $0.00 \%$ & $0.00 \%$ & $100.00 \%$ & 0.0 & $0.00 \%$ & $0.00 \%$ & $100.00 \%$ & $100 \%$ \\
\hline $11 / 2$ in & 0.0 & $0.00 \%$ & $0.00 \%$ & $100.00 \%$ & 0.0 & $0.00 \%$ & $0.00 \%$ & $100.00 \%$ & $100 \%$ \\
\hline 1 in & 0.0 & $0.00 \%$ & $0.00 \%$ & $100.00 \%$ & 0.0 & $0.00 \%$ & $0.00 \%$ & $100.00 \%$ & $100 \%$ \\
\hline $3 / 4$ in & 265.1 & $3.21 \%$ & $3.21 \%$ & $96.79 \%$ & 255.1 & $3.07 \%$ & $3.07 \%$ & $96.93 \%$ & $97 \%$ \\
\hline $1 / 2$ in & 4418.7 & $53.44 \%$ & $56.65 \%$ & $43.35 \%$ & 4022.8 & $48.44 \%$ & $51.51 \%$ & $48.49 \%$ & $46 \%$ \\
\hline $3 / 8$ in & 2306.2 & $27.89 \%$ & $84.54 \%$ & $15.46 \%$ & 2544.6 & $30.64 \%$ & $82.15 \%$ & $17.85 \%$ & $17 \%$ \\
\hline No. 4 & 1017.2 & $12.30 \%$ & $96.85 \%$ & $3.15 \%$ & 1076.2 & $12.96 \%$ & $95.10 \%$ & $4.90 \%$ & $4 \%$ \\
\hline No. 8 & 177.5 & $2.15 \%$ & $98.99 \%$ & $1.01 \%$ & 285.8 & $3.44 \%$ & $98.55 \%$ & $1.45 \%$ & $1 \%$ \\
\hline Pan & 83.1 & $1.01 \%$ & $100.00 \%$ & & 120.8 & $1.45 \%$ & $100.00 \%$ & & \\
\hline Total & 8267.8 & $100.00 \%$ & & & 8305.3 & & & & \\
\hline & & & ASTM C 117 & 7: Minus 75 um & (No. 200) & & & & \\
\hline OD Mass, $\mathrm{g}$ & 9427.7 & Mass Aft, $\mathrm{g}$ & 9359.2 & Mass Loss, g & 68.5 & & & \% Loss: & $0.73 \%$ \\
\hline & & & STM C 127: Bulk & Specific Gravit & ty \& Absorpti & & & & \\
\hline & & & & & & & Run 1 & Run 2 & Avg \\
\hline & & SSD N & Mass in Air, $\mathrm{g}$ & & & & 3142.6 & 3529.7 & \\
\hline & & SSD Ma & ass in Water, $\mathrm{g}$ & & & & 1965.1 & 2207.5 & \\
\hline & & Displa & aced Water, $\mathrm{g}$ & & & & 1177.5 & 1322.2 & \\
\hline & & Wat & ter Temp C & & & & 22.7 & 22.7 & \\
\hline & Rel & ative Density (B & Bulk Specific Gra & avity)(SSD) & & & 2.669 & 2.670 & 2.67 \\
\hline & & Oven & Dry Mass, $\mathrm{g}$ & & & & 3123.4 & 3508.8 & \\
\hline & & Mois & sture Loss, $\mathrm{g}$ & & & & 19.2 & 20.9 & \\
\hline & & & bsorption & & & & $0.61 \%$ & $0.60 \%$ & $0.61 \%$ \\
\hline & & ASTM & M C 88 Magnesil & um Sulfate Sour & undness (5 cy & (cles): & & & \\
\hline & Gradation $>5 \%$ & OD Mass, g & Mass Aft, $\mathrm{g}$ & Mass Loss, $\mathrm{g}$ & $\%$ Loss & Wt'ed \% & & & \\
\hline $21 / 2$ in & $0.00 \%$ & 1.0 & 1.0 & 0.0 & 0.00 & $0.0 \%$ & & & \\
\hline 2 in & $0.00 \%$ & 1.0 & 1.0 & 0.0 & 0.00 & $0.0 \%$ & & & \\
\hline $11 / 2$ in & $0.00 \%$ & 1.0 & 1.0 & 0.0 & 0.00 & $0.0 \%$ & & & \\
\hline 1 in & $0.00 \%$ & 1.0 & 1.0 & 0.0 & 0.00 & $0.0 \%$ & & & \\
\hline $3 / 4$ in & $3.21 \%$ & 674.4 & 668.0 & 6.4 & 0.01 & $0.0 \%$ & & & \\
\hline $1 / 2$ in & $53.44 \%$ & 674.4 & 668.0 & 6.4 & 0.01 & $0.5 \%$ & & & \\
\hline $3 / 8$ in & $27.89 \%$ & 1004.6 & 978.6 & 26.0 & 0.03 & $0.7 \%$ & & & \\
\hline No. 4 & $12.30 \%$ & 280.9 & 280.9 & 0.0 & 0.00 & $0.0 \%$ & & & \\
\hline No. 8 & $2.15 \%$ & 280.9 & 280.9 & 0.0 & 0.00 & $0.0 \%$ & & & \\
\hline Total & $98.99 \%$ & & & & & $1.3 \%$ & & & $1.3 \%$ \\
\hline & & ASTM C & 123 Light Weig & ght Particles and & d Pieces in A & ggregate & & & \\
\hline Zinc Bromide 2 & 40 sp.gr & & No mat'l. lighter & r than $2.00 \mathrm{sp} . \mathrm{g}$ & & & & & \\
\hline OD Mass, $\mathrm{g}$ & 3004.0 & Ret Mass, $\mathrm{g}$ & 2959.7 & Mass Loss, $\mathrm{g}$ & 44.3 & \% Loss: & $1.47 \%$ & & $1.5 \%$ \\
\hline & & ASTM & $\mathrm{M} \mathrm{C131}$ or C535 & LA Abrasion Re & esistance $(50$ & $0 \mathrm{rev})$ & & & \\
\hline Grading: & $\mathrm{B}$ & OD Mass, $\mathrm{g}$ & 5009.9 & Ret Mass, $\mathrm{g}$ & 3903.8 & \% Loss: & $22.08 \%$ & & $22.1 \%$ \\
\hline & & & ASTM C 142: Cla & ay Lumps \& Fri & iable Particles & & & & \\
\hline & Gradation $>5 \%$ & OD Mass, g & Mass Aft, $\mathrm{g}$ & Mass Loss, $\mathrm{g}$ & $\%$ Loss & Wt'ed \% & & & \\
\hline $21 / 2$ in & $0.00 \%$ & 1.0 & 1.0 & 0.0 & 0.00 & $0.0 \%$ & & & \\
\hline 2 in & $0.00 \%$ & 1.0 & 1.0 & 0.0 & 0.00 & $0.0 \%$ & & & \\
\hline $11 / 2$ in & $0.00 \%$ & 1.0 & 1.0 & 0.0 & 0.00 & $0.0 \%$ & & & \\
\hline 1 in & $0.00 \%$ & 1.0 & 1.0 & 0.0 & 0.00 & $0.0 \%$ & & & \\
\hline $3 / 4$ in & $3.21 \%$ & 2037.7 & 2035.4 & 2.3 & 0.00 & $0.0 \%$ & & & \\
\hline $1 / 2$ in & $53.44 \%$ & 2037.7 & 2035.4 & 2.3 & 0.00 & $0.1 \%$ & & & \\
\hline $3 / 8$ in & $27.89 \%$ & 2001.1 & 1999.2 & 1.9 & 0.00 & $0.0 \%$ & & & \\
\hline No. 4 & $12.30 \%$ & 1007.0 & 1002.4 & 4.6 & 0.00 & $0.1 \%$ & & & \\
\hline Total & $96.85 \%$ & & & & & $0.1 \%$ & & & $0.1 \%$ \\
\hline & & & ASTM D 4791 & Flat or Elongate & ed Particles: & & & & \\
\hline 3:1 Ratio & Gradation $>10 \%$ & Pcs Before & Flat Pcs & Elong Pcs & F\&E PCS & $\%$ F,E\&FE & Wt'ed \% & & \\
\hline $21 / 2$ in & $0.00 \%$ & 100 & 0 & 0 & 0 & 0.00 & $0.0 \%$ & & \\
\hline 2 in & $0.00 \%$ & 100 & 0 & 0 & 0 & 0.00 & $0.0 \%$ & & \\
\hline $11 / 2$ in & $0.00 \%$ & 100 & 0 & 0 & 0 & 0.00 & $0.0 \%$ & & \\
\hline 1 in & $0.00 \%$ & 100 & 0 & 0 & 0 & 0.00 & $0.0 \%$ & & \\
\hline $3 / 4$ in & $3.21 \%$ & 100 & 0 & 0 & 0 & 0.00 & $0.0 \%$ & & \\
\hline $1 / 2$ in & $53.44 \%$ & 107 & 0 & 0 & 0 & 0.00 & $0.0 \%$ & & \\
\hline $3 / 8$ in & $27.89 \%$ & 120 & 0 & 0 & 0 & 0.00 & $0.0 \%$ & & \\
\hline No. 4 & $12.30 \%$ & 102 & 0 & 0 & 0 & 0.00 & $0.0 \%$ & & \\
\hline Total & $96.85 \%$ & & & & & & $0.0 \%$ & & 0.0 \\
\hline
\end{tabular}




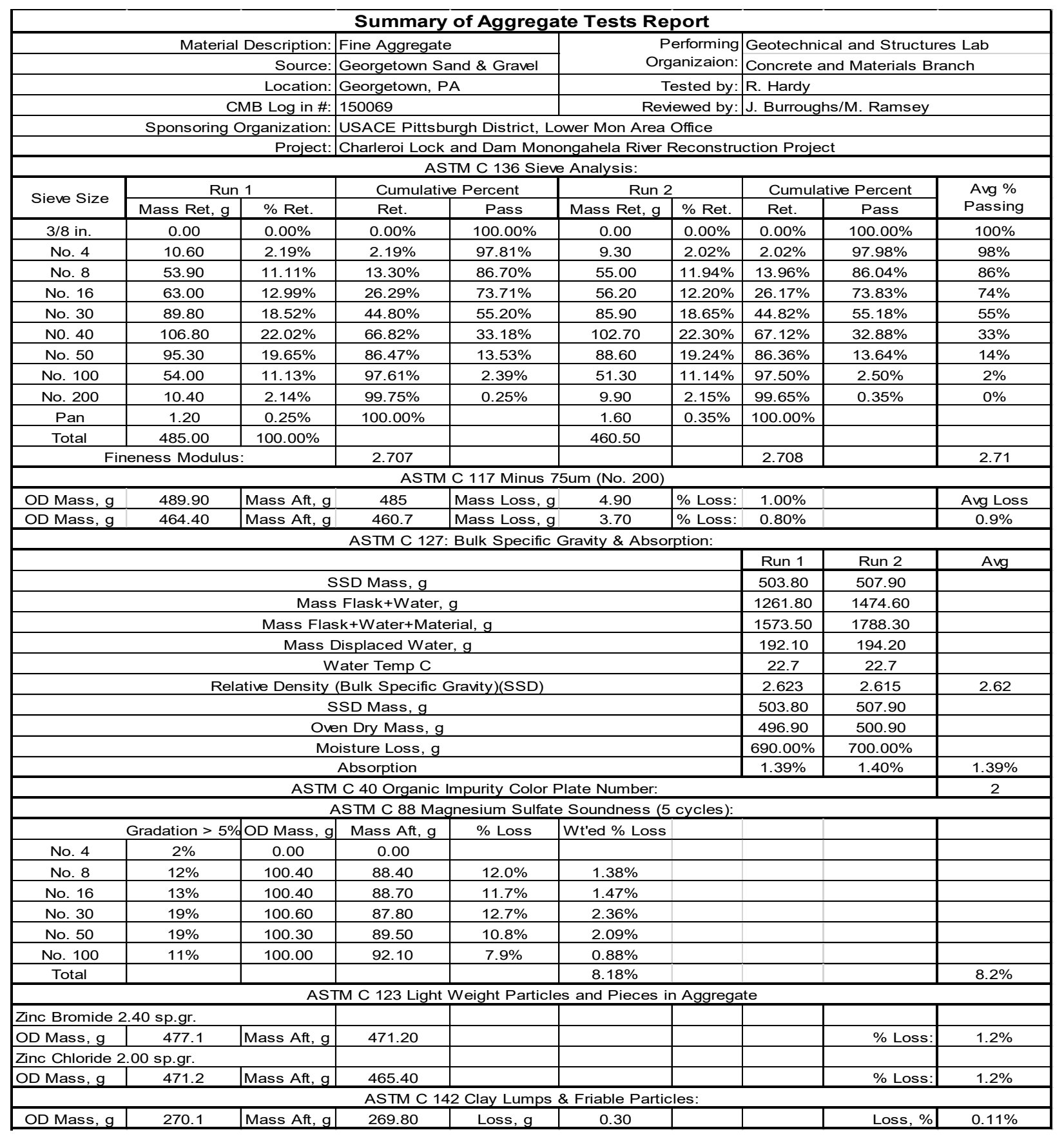




\begin{tabular}{|c|c|c|c|c|c|c|c|c|c|}
\hline \multicolumn{10}{|c|}{ Summary of Aggregate Tests Report } \\
\hline \multicolumn{3}{|c|}{ Material Description: } & \multicolumn{2}{|l|}{ Fine Aggregate } & \multirow{2}{*}{\multicolumn{2}{|c|}{ Performing Organizaion: }} & \multirow{2}{*}{\multicolumn{3}{|c|}{$\begin{array}{l}\text { Geotechnical and Structures Lab } \\
\text { Concrete and Materials Branch }\end{array}$}} \\
\hline & & Source: & \multicolumn{2}{|l|}{ Shelly Materials } & & & & & \\
\hline & & Location: & \multicolumn{2}{|l|}{ Thornville, $\mathrm{OH}$} & \multicolumn{2}{|r|}{ Tested by: } & \multicolumn{3}{|l|}{ R. Hardy } \\
\hline & & CMB Log in \#: & 160004 & & \multicolumn{2}{|c|}{ Reviewed by: } & \multicolumn{3}{|c|}{ J. Burroughs/M. Ramsey } \\
\hline \multicolumn{3}{|c|}{ Sponsoring Organization: } & \multicolumn{7}{|c|}{ USACE Pittsburgh District, Lower Mon Area Office } \\
\hline \multirow{2}{*}{\multicolumn{3}{|c|}{ Project: }} & \multicolumn{7}{|c|}{ Charleroi Lock and Dam Monongahela River Reconstruction Project } \\
\hline & & & \multicolumn{4}{|c|}{ ASTM C 136 Sieve Analysis: } & & & \\
\hline Sieve Size & Mass Ret, g & $\%$ Ret. & Ret. & Pass & Mass Ret, g & $\%$ Ret. & Ret. & Pass & Passing \\
\hline $3 / 8$ in. & 0.00 & $0.00 \%$ & $0.00 \%$ & $100.00 \%$ & 0.00 & $0.00 \%$ & $0.00 \%$ & $100.00 \%$ & $100 \%$ \\
\hline No. 4 & 5.90 & $1.09 \%$ & $1.09 \%$ & $98.91 \%$ & 6.10 & $1.11 \%$ & $1.11 \%$ & $98.89 \%$ & $99 \%$ \\
\hline No. 8 & 55.60 & $10.27 \%$ & $11.36 \%$ & $88.64 \%$ & 57.90 & $10.56 \%$ & $11.67 \%$ & $88.33 \%$ & $88 \%$ \\
\hline No. 16 & 60.90 & $11.25 \%$ & $22.61 \%$ & $77.39 \%$ & 59.10 & $10.78 \%$ & $22.45 \%$ & $77.55 \%$ & $77 \%$ \\
\hline No. 30 & 112.50 & $20.78 \%$ & $43.39 \%$ & $56.61 \%$ & 112.60 & $20.53 \%$ & $42.98 \%$ & $57.02 \%$ & $57 \%$ \\
\hline NO. 40 & 146.50 & $27.06 \%$ & $70.45 \%$ & $29.55 \%$ & 150.40 & $27.43 \%$ & $70.40 \%$ & $29.60 \%$ & $30 \%$ \\
\hline No. 50 & 98.60 & $18.21 \%$ & $88.66 \%$ & $11.34 \%$ & 98.30 & $17.92 \%$ & $88.33 \%$ & $11.67 \%$ & $12 \%$ \\
\hline No. 200 & 8.50 & $1.57 \%$ & $99.85 \%$ & $0.15 \%$ & 8.80 & $1.60 \%$ & $99.73 \%$ & $0.27 \%$ & $0 \%$ \\
\hline Pan & 0.80 & $0.15 \%$ & $100.00 \%$ & & 1.50 & $0.27 \%$ & $100.00 \%$ & & \\
\hline Total & 541.40 & $100.00 \%$ & & & 548.40 & $100.00 \%$ & & & \\
\hline & ieness Modulu & & 2.654 & & & & 2.647 & & 2.65 \\
\hline & & & ASTM C & 117 Minus 75 & sum (No. 200) & & & & \\
\hline OD Mass, g & 544.40 & Mass Aft, $\mathrm{g}$ & 541.4 & Mass Loss, g & 3.00 & \% Loss: & $0.55 \%$ & & Avg Loss \\
\hline OD Mass, g & 551.90 & Mass Aft, $\mathrm{g}$ & 548.4 & Mass Loss, $\mathrm{g}$ & 3.50 & \% Loss: & $0.63 \%$ & & $0.6 \%$ \\
\hline & & & ASTM C 127: B & Bulk Specific G & Sravity \& Absorp & tion: & & & \\
\hline & & & & & & & Run 1 & Run 2 & Avg \\
\hline & & & D Mass, $\mathrm{g}$ & & & & 508.60 & 505.10 & \\
\hline & & Mass $\mathrm{F}$ & Flask+Water, g & & & & 1263.50 & 1479.20 & \\
\hline & & Mass Flask & $\mathrm{L}+$ Water+Materi & ial, g & & & 1576.30 & 1790.10 & \\
\hline & & Mass Dis & splaced Water, & & & & 195.80 & 194.20 & \\
\hline & & Wa & ater Temp C & & & & 21.8 & 21.8 & \\
\hline & & lative Density (B & 3ulk Specific Gr & ravity)(SSD) & & & 2.598 & 2.601 & 2.60 \\
\hline & & Oven & Dry Mass, $\mathrm{g}$ & & & & 499.90 & 496.60 & \\
\hline & & ASTM C & C 40 Organic Im & apurity Color $\mathrm{Pl}$ & late Number: & & & & 2 \\
\hline ASTM C $88 \mathrm{Mc}$ & gnesium Sulfat & e Soundness (5 & cycles): & New Solutio & on 1.299 sp.gr. & & & & \\
\hline Gradatic & $1>5 \%$ & OD Mass, $\mathrm{g}$ & Mass Aft, g & $\%$ Loss & Wt'ed \% Loss & & & & \\
\hline No. 4 & $1 \%$ & & & $11.1 \%$ & $0.12 \%$ & & & & \\
\hline No. 8 & $10 \%$ & 100.00 & 88.90 & $11.1 \%$ & $1.16 \%$ & & & & \\
\hline No. 16 & $11 \%$ & 100.00 & 86.80 & $13.2 \%$ & $1.45 \%$ & & & & \\
\hline No. 30 & $21 \%$ & 100.10 & 81.90 & $18.2 \%$ & $3.76 \%$ & & & & \\
\hline Nos. $40 \& 50$ & $45 \%$ & 100.10 & 90.70 & $9.4 \%$ & $4.25 \%$ & & & & \\
\hline No. 100 & $10 \%$ & & & & & & & & \\
\hline Minus No. 100 & $2 \%$ & & & & & & & & \\
\hline Total & $100 \%$ & & & & $10.62 \%$ & & & & $10.6 \%$ \\
\hline & & ASTM & A C 123 Light $\mathrm{W}$ & Veight Particles & 5 and Pieces in & Aggregate & & & \\
\hline Zinc Bromide 2 & 40 sp.gr. & & & & & & & & \\
\hline OD Mass, $\mathrm{g}$ & 531.5 & Mass Aft, g & 525.60 & & & & & \% Loss: & $1.11 \%$ \\
\hline Zinc Chloride 2 & 0 sp.gr. & & & & & & & & \\
\hline OD Mass, $\mathrm{g}$ & 535.3 & Mass Aft, $\mathrm{g}$ & 531.40 & & & & & \% Loss: & $0.73 \%$ \\
\hline & & & ASTM C 142 & Clay Lumps \& & Friable Particl & es: & & & \\
\hline OD Mass, g & 156.2 & Mass Aft, g & 155.80 & Loss, g & 0.40 & & & Loss, \% & $0.26 \%$ \\
\hline
\end{tabular}




\begin{tabular}{|c|c|c|c|c|c|c|c|c|c|}
\hline \multirow{3}{*}{ 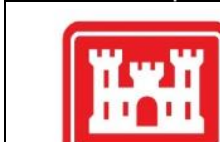 } & II & \multicolumn{3}{|c|}{$\begin{array}{l}\text { Report on Test } \\
\text { Specifications: }\end{array}$} & \multicolumn{5}{|c|}{$\begin{array}{l}\text { ASTM C1271 and C1301 for chemical properties and ASTM } \\
\text { D546 and D4318 for physical properties of limestone powder. }\end{array}$} \\
\hline & I & \multicolumn{5}{|c|}{ Performing Orgnaization: } & \multicolumn{3}{|c|}{ Sponsoring Orgnaization: } \\
\hline & & \multicolumn{5}{|c|}{ U.S Army Engineer Research and Development Center } & \multicolumn{3}{|c|}{ U.S. Army Corps of Engineers } \\
\hline \multirow{3}{*}{\multicolumn{2}{|c|}{$\begin{array}{l}\text { US Army Corps of Engineers } \\
\text { BUILDING STRONG. }\end{array}$}} & \multicolumn{5}{|c|}{ Geotechnical and Structures Laboratory (CEERD-GMC) } & \multicolumn{3}{|c|}{ Pittsburgh District, Lower Mon Area Office } \\
\hline & & \multicolumn{5}{|c|}{3909 Halls Ferry Road } & \multicolumn{3}{|c|}{1811 Schoonmaker Ave., } \\
\hline & & \multicolumn{3}{|c|}{ Vicksburg, MS 39180-6199 } & & & \multicolumn{3}{|c|}{ Monesson, PA 15062} \\
\hline \multicolumn{10}{|c|}{ Material Description: Limestone Powder } \\
\hline \multicolumn{5}{|c|}{ Manufacturer: Graymont Limestone } & & & & & \\
\hline \multicolumn{2}{|r|}{ Location: } & \multicolumn{3}{|c|}{ Pleasant Gap, PA } & & & & & \\
\hline \multicolumn{5}{|c|}{ CMB Serial \#: 150067} & & & & & \\
\hline \multirow{2}{*}{\multicolumn{5}{|c|}{$\begin{array}{l}\text { Project: Charleroi Lock and Dam Mononga } \\
\text { Chemical Analysis }\end{array}$}} & ahela River Re & constructio & n Project & & \\
\hline & & & & & \multicolumn{5}{|c|}{ Test Results } \\
\hline \multicolumn{5}{|c|}{$\mathrm{SiO}_{2}, \%$} & \multicolumn{5}{|c|}{0.95} \\
\hline \multicolumn{5}{|c|}{$\mathrm{Al}_{2} \mathrm{O}_{3}, \%$} & \multicolumn{5}{|c|}{0.38} \\
\hline & & $\mathrm{O}_{3}, \%$ & & & & & 0.15 & & \\
\hline & & $0, \%$ & & & & & 54.18 & & \\
\hline & & $20, \%$ & & & & & 0.04 & & \\
\hline & & $20, \%$ & & & & & 0.09 & & \\
\hline & & gO, \% & & & & & 0.71 & & \\
\hline & & , \% & & & & & 0.17 & & \\
\hline & Loss on ign & ition $\left(950^{\circ} \mathrm{C}\right)$ & $\%$ & & & & 43.20 & & \\
\hline & $\underline{\text { Calculate }}$ & Compound & & & & & & & \\
\hline & $\mathrm{Ca}$ as & $\mathrm{CaCO}_{3}, \%$ & & & & & 96.65 & & \\
\hline & $\mathrm{Mg}$ as & $\mathrm{MgCO}_{3}, \%$ & & & & & 1.49 & & \\
\hline & Calculated $\mathrm{Ca}$ & arbonates as & $\mathrm{CO}_{2}$ & & & & 43.20 & & \\
\hline & L.O.I. / C & $\mathrm{O}_{2}$ Balance & & & & & 1 & & \\
\hline Calct & ulated Compo & unds- $\mathrm{Mg}$ as & Dolomite & & & & & & \\
\hline & $\mathrm{Mg}$ as $\mathrm{Ca}$ & $\mathrm{Mg}\left(\mathrm{CO}_{3) 2}, 9\right.$ & & & & & 3.26 & & \\
\hline & Residual Ce & as $\mathrm{CaCO}_{3}$ & & & & & 94.92 & & \\
\hline & & STM D 546 & Sieve Analy & $y$ sis of Miner & ral Filler for $\mathrm{Bi}$ & tuminous $\mathrm{P}$ & aving Mixtur & & \\
\hline & Run & & Cumulativ & e Percent & Run & & Cumulativ & Percent & \\
\hline Sieve Size & $\begin{array}{l}\text { Mass } \\
\text { Retained, } g\end{array}$ & \begin{tabular}{c|c}
$\%$ \\
Retained
\end{tabular} & \begin{tabular}{c|c}
$\%$ \\
Retained
\end{tabular} & $\%$ Passing & $\begin{array}{c}\text { Mass } \\
\text { Retained, g }\end{array}$ & $\begin{array}{c}\% \\
\text { Retained }\end{array}$ & $\begin{array}{c}\% \\
\text { Retained }\end{array}$ & $\begin{array}{c}\% \\
\text { Passing }\end{array}$ & Avg. $\%$ Passing \\
\hline No. 16 & 0.00 & $0.00 \%$ & $0.00 \%$ & $100.00 \%$ & 0.00 & $0.00 \%$ & $0.00 \%$ & $100.00 \%$ & $100.00 \%$ \\
\hline No. 30 & 0.52 & $0.51 \%$ & $0.51 \%$ & $99.49 \%$ & 0.60 & $0.53 \%$ & $0.53 \%$ & $99.47 \%$ & $99.48 \%$ \\
\hline No. 50 & 0.50 & $0.49 \%$ & $1.00 \%$ & $99.00 \%$ & 0.58 & $0.51 \%$ & $1.04 \%$ & $98.96 \%$ & $98.98 \%$ \\
\hline No. 100 & 1.93 & $1.89 \%$ & $2.89 \%$ & $97.11 \%$ & 2.08 & $1.83 \%$ & $2.87 \%$ & $97.13 \%$ & $97.12 \%$ \\
\hline No. 200 & 22.00 & $21.56 \%$ & $24.45 \%$ & $75.55 \%$ & 24.16 & $21.25 \%$ & $24.12 \%$ & $75.88 \%$ & $75.72 \%$ \\
\hline Minus No.200 & 77.09 & $75.55 \%$ & $100.00 \%$ & & 86.28 & $75.88 \%$ & $100.00 \%$ & & \\
\hline Original Sample & 102.04 & $100.00 \%$ & & & 113.70 & $100.00 \%$ & & & \\
\hline
\end{tabular}




\title{
A.3 Admixtures materials
}

\author{
The Euclid Chemical Company
}

\section{EUCON AEA-92}

Air Entraining Agent For Concrete

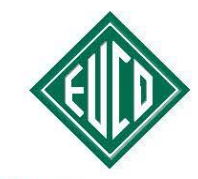

EUCLID CHEMICAL

\section{DESCRIPTION}

EUCON AEA-92 is formulated for use as an air entraining admixture for concrete of all types and is manufactured under rigid control which assures uniform and precise performance. It should be added to the mix independently and not with other admixtures.

\section{Primary ApPLICATIONS}

- Ready mix concrete

- Structural concrete

- Mass concrete

- Paving concrete

- All exterior concrete

\section{FEATURES/BENEFITS}

- Provides a stable air void system with proper bubble size and spacing. This air void system protects concrete against damage caused by repeated freeze/thaw cycles

- Concrete is made more resistant to de-icing salts, sulfate attack and corrosive water

- Less mixing water can be used per yard (meter) of concrete and placeability is improved

- Minimizes bleeding and segregation of the concrete

\section{TECHNICAL INFORMATION}

EUCON AEA-92 is an aqueous solution compound of synthetic organic chemicals. It is compatible with concrete mixes containing calcium chloride, water reducing admixtures, retarding admixtures, or high range water reducers.

\section{PACKAGING}

EUCON AEA-92 is packaged in bulk, 275 gal $(1041 \mathrm{~L})$ totes, 55 gal (208 L) drums and 5 gal $(18.9 \mathrm{~L})$ pails.

\section{SHELF LIFE}

2 years in original, unopened package.

\section{SPECIFICATIONS/COMPLIANCES}

EUCON AEA-92 meets or exceeds the requirements of the following specifications:

- Corps of Engineers Specification CRD C-13

- ASTM Specification C 260

- AASHTO Specification M 154

- ANSI/NSF STD 61 


\section{DIRECTIONS FOR USE}

EUCON AEA-92 is typically dosed at a rate of 0.1 to 4 oz per $100 \mathrm{lbs}$ ( 6 to $260 \mathrm{~mL}$ per $100 \mathrm{~kg}$ ) of total cementitious material to entrain 3\% - $6 \%$ air content. The amount of EUCON AEA-92 will vary depending on type of cement, fineness of sand, temperature, design of the mix, other admixtures, etc. Concrete mixes must be tested regularly to confirm that proper air content is achieved. EUCON AEA 92 should be added directly to the sand to achieve maximum performance.

\section{Precautions/Limitations}

- Protect EUCON AEA-92 from freezing.

- Consult your local Euclid Chemical representative for the proper dosage rate adjustments when using fly ash, slag or high range water reducers.

- Add to the mix independent of other admixtures.

- In all cases, consult the Safety Data Sheet before use. 


\section{The Euclid Chemical Company}

\section{EUCON RETARDER 75}

\section{Concrete Water Reducing Set Controlling Retarder}

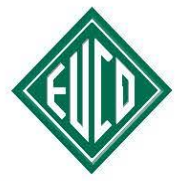

EUCLID CHEMICAL

\section{DESCRIPTION}

EUCON RETARDER 75 is a synthetically produced liquid water-reducing and set retarding admixture for concrete. EUCON RETARDER 75 does not contain calcium chloride or other potential corroding materials, and may be used in the presence of aluminum or zinc metals. It is compatible with air-entraining agents, water reducers and calcium chloride, but they must be added separately to the mix.

\section{Primary APPLICATIONS}

- Prestressed concrete

- Concrete requiring water reduction and set time control

- Architectural concrete

- Hot weather concrete placement

\section{FEATURES/BENEFITS}

Plastic Concrete

- Retards setting characteristics

- Improves finishability

- Improves workability

- Reduces water requirements

- Reduces segregation

Hardened Concrete

- Increases strengths

- Improves finished appearance

- Reduces cracking

- Reduces permeability

- Non staining

\section{TECHNICAL INFORMATION}

Perfomance Data

The following test results were achieved using typical ASTM C $494 \mathrm{mix}$ design requirements, $517 \mathrm{lb} / \mathrm{yd}^{3}\left(307 \mathrm{~kg} / \mathrm{m}^{3}\right)$ cement content and similar ( \pm 0.5$) \%$ air content.

These results were obtained under laboratory conditions with materials and mix designs meeting the specifications of ASTM C 494. Changes in materials and mix designs can affect the dosage response of EUCON RETARDER 75.
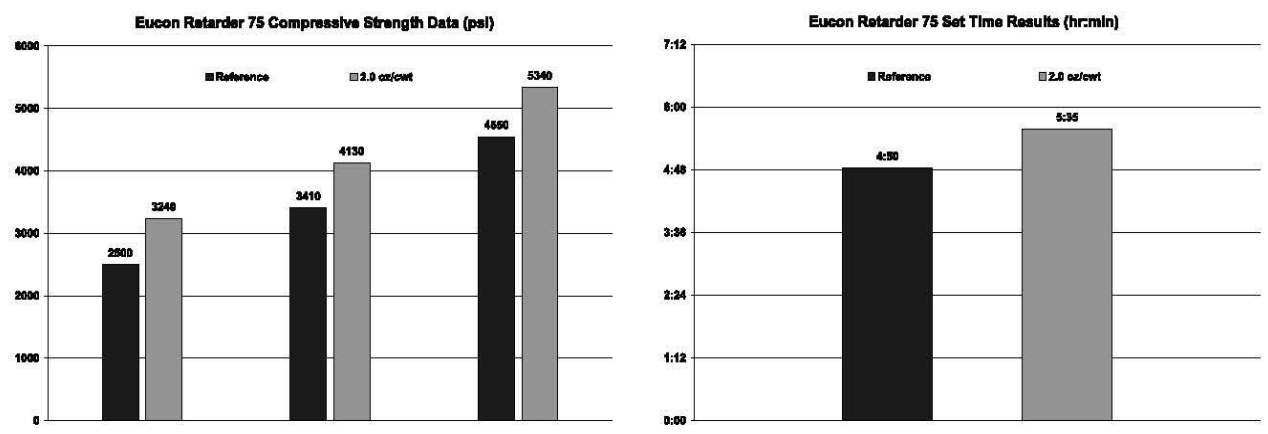
PACKAgINg

EUCON RETARDER 75 is packaged in bulk, $275 \mathrm{gal}(1041 \mathrm{~L})$ totes, $55 \mathrm{gal}(208 \mathrm{~L})$ drums and $5 \mathrm{gal}(18.9 \mathrm{~L})$ pails.

\section{SHELF LIFE}

1 year in original, unopened container.

\section{SPECIFICATIONS/COMPLIANCES}

- ASTM C 494, Types B \& D

- AASHTO M 194

- ANSI/NSFSTD 61

\section{DIRECTIONS FOR USE}

EUCON RETARDER 75 is normally used at dosages of 2 to 5 oz per $100 \mathrm{lb}$ (130 to $330 \mathrm{ml}$ per $100 \mathrm{~kg}$ ) of cementitious material, depending on the application. Higher dosages are acceptable with prior testing and confirmation of the desired performance with specific materials being used.

EUCON RETARDER 75 should be added to the initial batch water of the concrete mixture. Do not dispense onto dry cement.

\section{Precautions/Limitations}

- Care should be taken to maintain EUCON RETARDER 75 above freezing; however, freezing and subsequent thawing will not harm the material if thoroughly agitated.

- Add to mix independent of other admixtures.

- In all cases, consult the Safety Data Sheet before sue. 


\section{The Euclid Chemical Company}

\section{EUCON WR}

\section{Water RedUCINg AdMiXtURE}

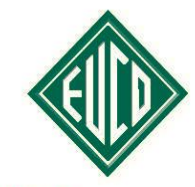

EUCLID CHEMICAL

\section{DESCRIPTION}

EUCON WR is an aqueous solution of refined lignosulfonate which is completely free of any added chloride ions. It is a water-reducing, normal-set admixture for concrete. It provides a more plastic and cohesive mix in the fresh concrete and better durability, reduced shrinkage and less permeability in the hardened concrete.

\section{PrIMARY APPLICATIONS}

- Ready mixed concrete

- Prestressed concrete

- General use concrete

- Precast concrete

- Lightweight concrete

- Expansive concrete

\section{FEATURES/BENEFITS}

- Provides easier handling and finishing

- Increases strength

- Provides increased durability

- Reduces shrinkage and permeability

\section{TECHNICAL INFORMATION}

\section{Perfomance Data}

The following test results were achieved using typical ASTM C 494 mix design requirements, $517 \mathrm{lb} / \mathrm{yd} \mathrm{d}^{3}$ $\left(307 \mathrm{~kg} / \mathrm{m}^{3}\right)$ cement content and similar $( \pm 0.5) \%$ air content.

These results were obtained under laboratory conditions with materials and mix designs meeting the specifications of ASTM C 494. Changes in materials and mix designs can affect the dosage response of EUCON WR.
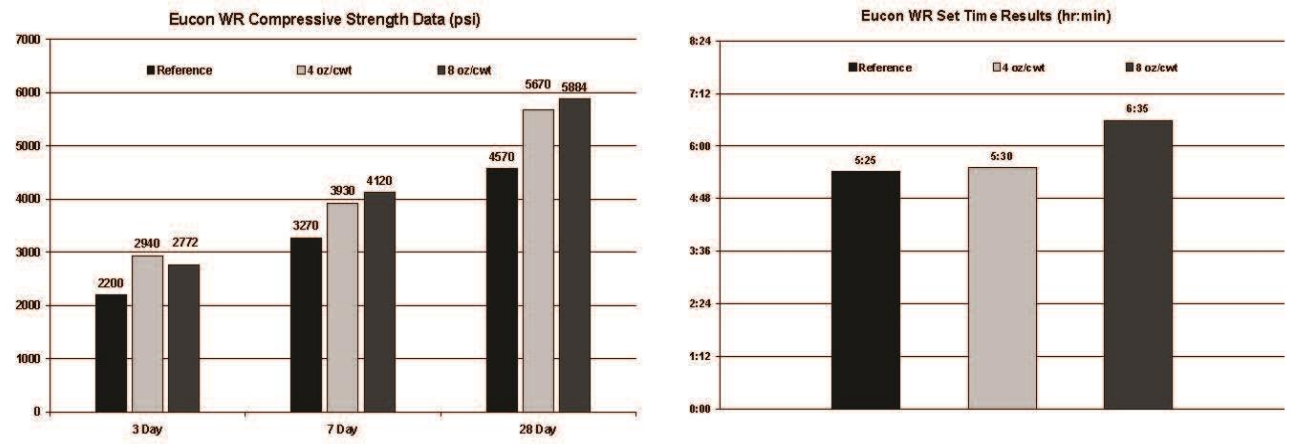


\section{Packaging}

EUCON WR is packaged in bulk, 275 gal $(1041 \mathrm{~L})$ totes, 55 gal $(208 \mathrm{~L})$ drums and $5 \mathrm{gal}(18.9 \mathrm{~L})$ pails.

\section{SHELF LIFE}

1 year in original, unopened container.

\section{SPECIFICATIONS/COMPLIANCES}

EUCON WR meets or exceeds the requirements of:

- ASTM C 494, Type A, Type B and Type D

- AASHTO M 194

\section{DIRECTIONS FOR USE}

EUCON WR is typically used at dosages of 2 to $10 \mathrm{fl} \mathrm{oz} / \mathrm{cwt}(130$ to $650 \mathrm{~mL} / 100 \mathrm{~kg}$ ) of cementitious materials. Higher dosages are acceptable with prior testing and confirmation of the desired performance with specific materials being used.

EUCON WR admixture has been tested as per ASTM C494 at a Type A dosage of $4 \mathrm{fl} \mathrm{oz} / \mathrm{cwt}(260 \mathrm{~mL} / 100 \mathrm{~kg})$ of cementitious materials and at $8 \mathrm{fl}$ oz/cwt $(520 \mathrm{~mL} / 100 \mathrm{~kg})$ of cementious materials for Type $B$ and D requirements.

Because of variations in job conditions and concrete materials, dosages other than the recommended amounts may be required. In such cases, contact your local Euclid sales representative.

EUCON WR should be added to the initial batch water of the concrete mixture. Do not dispense onto dry cement.

\section{Precautions/Limitations}

- Care should be taken to maintain EUCON WR above freezing; however, freezing and subsequent thawing

will not harm the material if thoroughly agitated.

- Add to mix independent of other admixtures.

- In all cases, consult the Safety Data Sheet before use. 


\section{The Euclid Chemical Company}

\section{EUCON AWA}

ANTI-WASHOUT ADMIXTURE

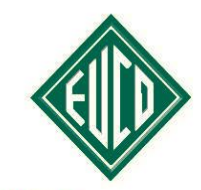

EUCLID CHEMICAL

\section{DESCRIPTION}

EUCON AWA is a ready to use liquid admixture designed to prevent the loss of cement and fine aggregate during the placement of underwater concrete. EUCON AWA is a blend of different powerful ingredients and colloidal agents that act primarily on the water preventing the cement paste from washing out during casting under water. EUCON AWA provides superior slump retention while greatly reducing the environmental impact due to cement wash out in below water applications.

\section{PRIMARY APPLICATIONS}

- Underwater bridge repair

- Dam repair below the waterline

- Underwater grouting and mortar application

- Damming underground rivers/lakes in mining operations

- Anti-segregation aid for use with lightweight and heavyweight aggregates

- Reduction or elimination of concrete bleed water for use with fast track construction

\section{FEATURES/Benefits}

- Minimal environmental impact due to cement washout

- Eliminates the need for expensive de-watering during underwater construction

- Greatly reduces or eliminates concrete bleed water

- Superior slump retention

- Does not effect water demand when slump is maintained

- Easily metered with standard admixture dispensing equipment

\section{TECHNICAL INFORMATION}

Appearance

EUCON AWA is a medium viscosity, dark brown liquid which will not discolor concrete

\section{PACKAgINg}

EUCON AWA is packaged in $275 \mathrm{gal}(1041 \mathrm{~L})$ totes, $55 \mathrm{gal}(208 \mathrm{~L})$ drums and $5 \mathrm{gal}(18.9 \mathrm{~L})$ pails.

\section{SHELF LIFE}

6 months in original, unopened container

\section{SPECIFICATIONS/COMPLIANCES}

- Meets ASTM C 494 Types S 


\section{DIRECTIONS FOR USE}

Underwater Applications

To reduce the washout of cement and fine aggregates when placing concrete underwater, 10 to $32 \mathrm{oz}$ per $100 \mathrm{lb}$ $(0.65$ to $2.1 \mathrm{~L} / 100 \mathrm{~kg})$ of cement is recommended. At a dosage rate of 25 oz per $100 \mathrm{lb}(1.6 \mathrm{~L} / 100 \mathrm{~kg})$ of cement, set retardation may be 6 to 10 hours. In non air entrained concrete applications, the concrete should be batched and the slump adjusted either with water or HRWR prior to the addition of EUCON AWA.

Do not use water to adjust slump after EUCON AWA has been dispensed, instead, adjust slump through the use of a superplasticizer such as EUCON 37. Contact Euclid Chemical for a product recommendation.

If air entrainment is desired, the addition of the air entraining admixture should be done at the beginning of the batching sequence. EUCON AWA should be added before the HRWR to insure an adequate air void system.

For further recommendations on underwater concreting and underwater concrete mix designs, please consult the $\mathrm{ACl} 304$, "Measuring, Mixing, Transporting and Placing Concrete".

\section{PrFCAUTIONS / LimITATIONS}

- Do not allow material to freeze

- Superplasticizers must be used to increase slump after the addition of EUCON AWA

- Significant set retardation may occur with the use of this product

- In all cases, consult the Safety Data Sheet before use. 


\section{The Euclid Chemical Company}

\section{EUCON 37}

High RANge WATER RedUCER - SUPERPLASTICIZER

\section{DESCRIPTION}

EUCON 37 is a high range water-reducing admixture. It may be added to the concrete at the job site or at the ready mix concrete plant. EUCON 37 is formulated to retain plastic consistancy for $30-60$ minutes after dosing depending on the initial slumps, dosage rates, and ambient temperature. No chlorides are used in its formulation; consequently, it is recommended for prestressed concrete. It is also compatible with air-entraining agents, waterproofing agents, calcium chloride and many other admixtures; however, each material should be added to the concrete separately.

\section{PrimaRY APPLICATIONS}

- High performance concrete

- General ready mix concrete

- Heavily reinforced concrete

- Flatwork and mass concrete

- Minimum water content concrete

- Low water/cement ratio concrete

- High slump, flowable concrete

\section{FEATURES/BENEFITS}

- Produces low water content and low water/cement ratio concrete allowing higher strengths

- Produces flowing concrete with better than normal strengths

- Aids in concrete placement and reduces labor cost

- When used in precast work with Type I cement will produce the high early strengths

\section{TECHNICAL INFORMATION}

\section{Performance Data:}

The following test results were achieved using typical ASTM C 494 mix design requirements, $517 \mathrm{lb} / \mathrm{yd}^{3}$ $\left(307 \mathrm{~kg} / \mathrm{m}^{3}\right)$ cement content and similar $( \pm 0.5) \%$ air content. These results were obtained under laboratory conditions with materials and mix designs meeting the specifications of ASTM C 494. Changes in materials and mix designs can affect the dosage response of EUCON 37.
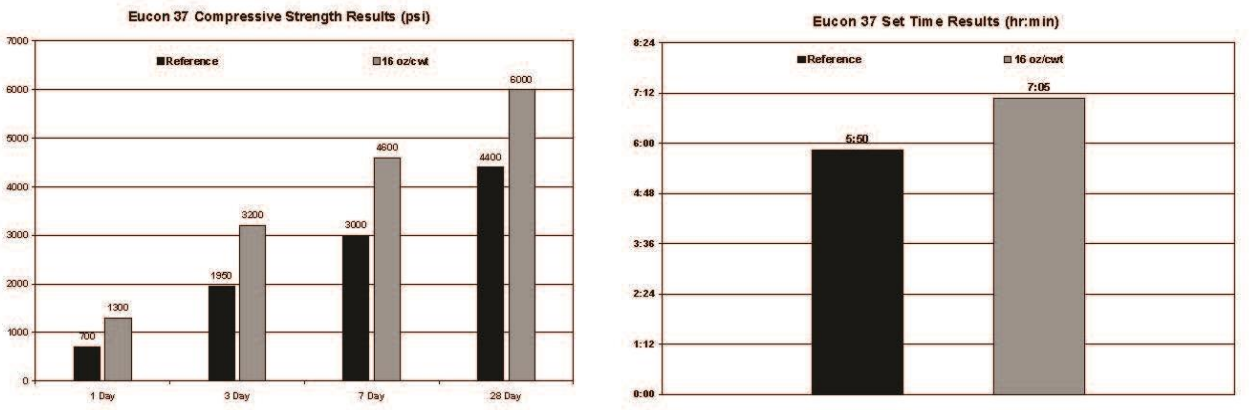


\section{Packaging}

EUCON 37 is packaged in bulk, 275 gal $(1041 \mathrm{~L})$ totes, 55 gal $(208 \mathrm{~L})$ drums and 5 gal (18.9 L) pails.

SHELF LIFE

2 years in original, unopened package.

\section{SPECIFICATIONS/COMPLIANCES}

- Fully complies with the requirements of ASTM C 494, Types A \& F admixtures.

- Fully complies with the requirements of AASHTO M 194.

\section{DIRECTIONS FOR USE}

EUCON 37 can be added to the initial batch water or directly on the freshly batched concrete and mixed for approximately 5 minutes or 70 revolutions. However, better results have been observed batching directly on the freshly batched concrete. It should not come into contact with dry cement or other admixtures until mixed thoroughly with the concrete batch.

EUCON 37 is typically used at dosages of 6 to 18 oz per $100 \mathrm{lbs}(400$ to $1170 \mathrm{~mL}$ per $100 \mathrm{~kg}$ ) of cementitious material. Other dosages are acceptable with prior testing and confirmation of the desired performance with specific materials being used.

For any concrete application including Self-Consolidating Concrete (SCC), the dosage of EUCON 37 will vary depending on the mix design, local materials, and individual needs of the concrete producer. Trial mixes should be run to verify plastic and hardened performance with local materials. If the material gradations are not optimum for SCC, a viscosity modifier may be used to improve the quality of the mix. Please consult a local Euclid Chemical Sales Professional for trial mixtures and dosage recommendations. EUCON 37 is compatible with most admixtures including air-entraining agents, accelerators, most water-reducers, retarders, shrinkage reducers, corrosion inhibitors, viscosity modifiers, and microsilica: however, each material should be added to the concrete separately.

Figure 1:Recommended Dosage of Eucon 37 to achieve flowable concrete $\left(7-9^{\prime \prime} / 180-230 \mathrm{~mm}\right.$ slump)

\begin{tabular}{|c|c|}
\hline Initial Slump, inches $(\mathbf{m m})$ & Dosage Range of Eucon 37, oz/cwt (mL/100 kg) \\
\hline $4(100)$ & $8-10(520-650)$ \\
\hline $3(75)$ & $10-12(650-780)$ \\
\hline $21 / 2(65)$ & $12-14(780-910)$ \\
\hline $2(50)$ & $14-16(910-1040)$ \\
\hline $11 / 2(40)$ & $16-18(1040-1170)$ \\
\hline
\end{tabular}

\section{Placement}

Concrete treated with EUCON 37 may be placed in the same fashion as conventional concrete.

\section{Formwork}

Forms for walls or narrow sections must be watertight, strong and have good bracing. During the "flowing period", when the concrete is at a slump of 7" to 9" (180-230 $\mathrm{mm})$, the concrete will exert a higher pressure at the base of the form than conventional concrete. Formwork for slabs is the same as for conventional concrete.

\section{Precautions / Limitations}

- Care should be taken to maintain EUCON 37 above freezing; however, freezing and subsequent thawing will not harm the material if thoroughly agitated. Never agitate with air or an air lance.

- Keep concrete from freezing until a minimum strength of 1000 psi $(7 \mathrm{MPa})$ is reached.

- In all cases, consult the Safety Data Sheet before use. 


\section{Appendix B: Worksheets for ASTM C1260}




\begin{tabular}{|c|c|c|c|c|c|c|c|c|c|c|c|c|c|c|c|c|}
\hline \multicolumn{17}{|c|}{\begin{tabular}{|l|l|} 
Mix: & Hanson Aggregate w/ Armstrong Cement \\
\end{tabular}} \\
\hline \multicolumn{17}{|c|}{ Potential Alkali Reactivity of Aggregates, ASTM C 1260} \\
\hline & Date & & \multicolumn{3}{|c|}{ Specimen 1} & \multicolumn{3}{|c|}{ Specimen 2} & \multicolumn{3}{|c|}{ Specimen 3} & \multicolumn{3}{|c|}{ Specimen 4} & Average & \multirow{2}{*}{\begin{tabular}{|l|} 
Standard \\
Deviation \\
\end{tabular}} \\
\hline \begin{tabular}{|l|} 
Mixture \\
\end{tabular} & & Age & \begin{tabular}{|l|} 
Reading \\
\end{tabular} & Change & Expansion & Reading & Change 1 & Expansion & Reading & Change & Expansion & Reading & Change & Expansion & Expansion & \\
\hline ID & $\mathrm{D} / \mathrm{M} / \mathrm{Yr}$ & (day) & (in.) & (in.) & (\%) & (in.) & (in.) & (\%) & (in.) & (in.) & (\%) & (in.) & (in.) & (\%) & (\%) & (\%) \\
\hline Mix & 4/28/2015 & & - & - & - & - & - & - & - & - & - & - & - & - & - & - \\
\hline Initial & $4 / 30 / 2015$ & & 0.0057 & & & 0.0052 & & & 0.0031 & & & 0.0029 & & & & \\
\hline zero read & $5 / 1 / 2015$ & 0 & 0.0103 & & 0.0000 & 0.0097 & & 0.0000 & 00078 & & 0.0000 & 0.0072 & & 0.0000 & 0.0000 & 0.0000 \\
\hline & $5 / 4 / 2015$ & 3 & 0.0141 & 0.0038 & 0.0380 & 0.0137 & 0.0040 & 0.0400 & 0.0116 & 0.0038 & 0.0380 & 0.0110 & 0.0038 & 0.0380 & 0.0385 & 0.0010 \\
\hline & $5 / 6 / 2015$ & 5 & 0.0179 & 0.0076 & 0.0760 & 0.0169 & 0.0072 & 0.0720 & 0.0132 & 0.0054 & 0.0540 & 0.0138 & 0.0066 & 0.0660 & 0.0670 & 0.0096 \\
\hline & 5/8/2015 & 7 & 0.0217 & 0.0114 & 0.1140 & 0.0209 & 0.0112 & 0.1120 & 00199 & 0.0121 & 0.1210 & 0.0174 & 0.0102 & 0.1020 & 0.1123 & 0.0078 \\
\hline & 5/15/2015 & 14 & 0.0262 & 0.0159 & 0.1590 & 0.0262 & 0.0165 & 0.1650 & 0.0257 & 0.0179 & 0.1790 & 0.0232 & 0.0160 & 0.1600 & 0.1658 & 0.0092 \\
\hline & 5/22/2015 & 21 & 0.0285 & 0.0182 & 0.1820 & 0.0275 & 0.0178 & 0.1780 & 0.0271 & 0.0193 & 0.1930 & 0.0242 & 0.0170 & 0.1700 & 0.1808 & 0.0096 \\
\hline & 5/29/2015 & 28 & 0.0298 & 0.0195 & 0.1950 & 0.0290 & 0.0193 & 0.1930 & 0.0275 & 0.0197 & 0.1970 & 0.0261 & 0.0189 & 0.1890 & 0.1935 & 0.0034 \\
\hline & $5 / 31 / 2015$ & 30 & 0.0302 & 0.0199 & \begin{tabular}{|l|}
0.1990 \\
\end{tabular} & 0.0290 & 0.0193 & 0.1930 & 0.0278 & 0.0200 & 0.2000 & 0.0268 & 0.0196 & 0.1960 & 0.1970 & 0.0032 \\
\hline
\end{tabular}

\section{ASTM C 1260 Armstrong Cement with Hanson Aggregate}

Charleroi Lock and Dam Project

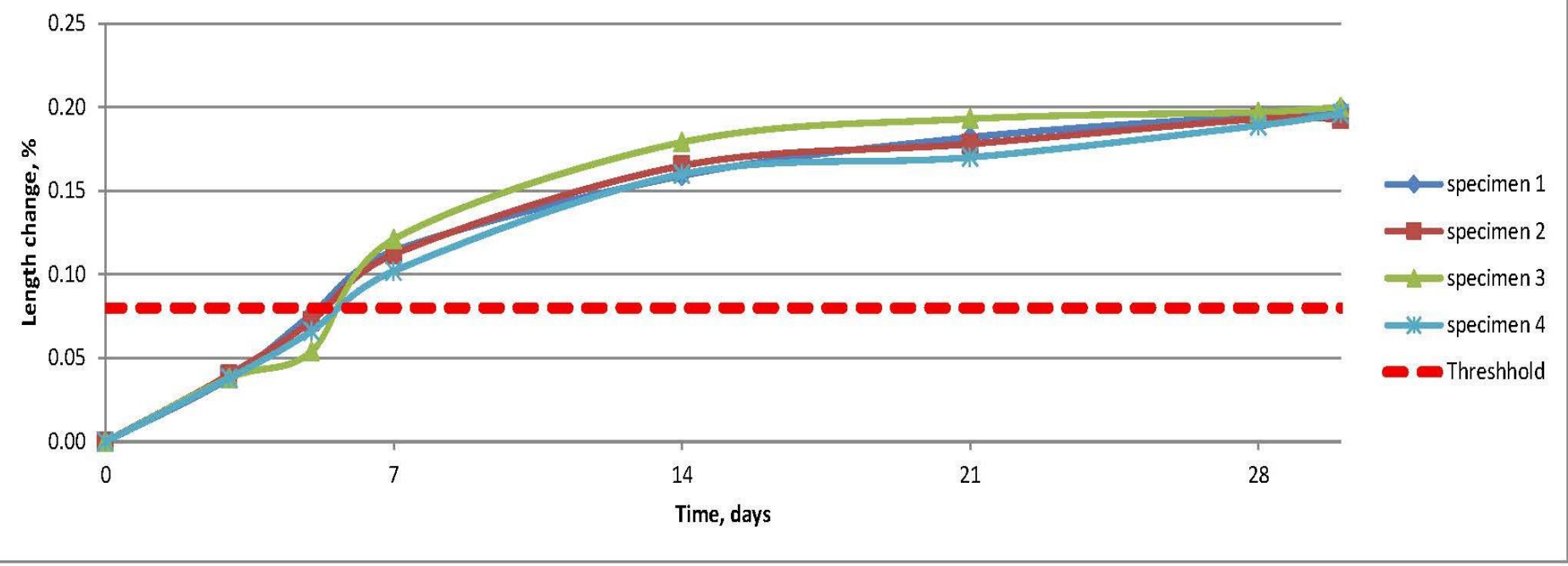




\begin{tabular}{|c|c|c|c|c|c|c|c|c|c|c|c|c|c|c|c|c|}
\hline \multicolumn{17}{|c|}{\begin{tabular}{l|l} 
Mix: & Georgetown Aggregate w/ Armstrong Cement
\end{tabular}} \\
\hline \multicolumn{17}{|c|}{ Potential Alkali Reactivity of Aggregates, ASTM C 1260} \\
\hline & Date & & \multicolumn{3}{|c|}{ Specimen 1} & \multicolumn{3}{|c|}{ Specimen 2} & \multicolumn{3}{|c|}{ Specimen 3} & \multicolumn{3}{|c|}{ Specimen 4} & Average & Standard \\
\hline Mixture & & Age $^{2}$ & Reading & Change & Expansion & Reading & Change & Expansion & Reading & Change & Expansion & Reading & Change & Expansior & Expansion & Deviation \\
\hline ID & $\mathrm{D} / \mathrm{M} / \mathrm{r}$ & (day) & (in.) & (in.) & $(\%)$ & (in.) & (in.) & $(\%)$ & (in.) & (in.) & $(\%)$ & (in.) & (in.) & $(\%)$ & $(\%)$ & $(\%)$ \\
\hline Mix & 4/21/2015 & & - & 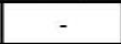 & - & - & - & - & - & - & - & - & - & - & - & - \\
\hline Initial & $4 / 23 / 2015$ & & -0.0014 & & & 0.0005 & & & -0.0032 & & & 0.0054 & & & & \\
\hline zero read & $4 / 24 / 2015$ & 0 & 0.0057 & & 0.0000 & 0.0076 & & 0.0000 & 0.0039 & & 0.0000 & 0.0122 & & 0.0000 & 0.0000 & 0.0000 \\
\hline & $4 / 27 / 2015$ & 3 & 0.0072 & 0.0015 & 0.0150 & 0.0094 & 0.0018 & 0.0180 & 0.0057 & 0.0018 & 0.0180 & 0.0141 & 0.0019 & 0.0190 & 0.0175 & 0.0017 \\
\hline & $4 / 29 / 2015$ & 5 & 0.0100 & 0.0043 & 0.0430 & 0.0124 & 0.0048 & 0.0480 & 0.0093 & 0.0054 & 0.0540 & 0.0171 & 0.0049 & 0.0490 & 0.0485 & 0.0045 \\
\hline & $5 / 1 / 2015$ & 7 & 0.0127 & 0.0070 & 0.0700 & 0.0151 & 0.0075 & 0.0750 & 0.0127 & 0.0088 & 0.0880 & 0.0197 & 0.0075 & 0.0750 & 0.0770 & 0.0077 \\
\hline & 5/8/2015 & 14 & 0.0206 & 0.0149 & 0.1490 & 0.0227 & 0.0151 & 0.1510 & 0.0194 & 0.0155 & 0.1550 & 0.0269 & 0.0147 & 0.1470 & 0.1505 & 0.0034 \\
\hline & $5 / 15 / 2015$ & 21 & 0.0216 & 0.0159 & 0.1590 & 0.0248 & 0.0172 & 0.1720 & 0.0214 & 0.0175 & 0.1750 & 0.0281 & 0.0159 & 0.1590 & \begin{tabular}{|c|}
0.1663 \\
\end{tabular} & 0.0085 \\
\hline & $5 / 22 / 2015$ & 28 & 0.0217 & 0.0160 & 0.1600 & 0.0248 & 0.0172 & 0.1720 & 0.0214 & 0.0175 & 0.1750 & 0.0281 & 0.0159 & 0.1590 & 0.1665 & 0.0082 \\
\hline & $5 / 24 / 2015$ & 30 & 0.0217 & 0.0160 & 0.1600 & 0.0250 & 0.0174 & 0.1740 & 0.0217 & 0.0178 & 0.1780 & 0.0283 & 0.0161 & 0.1610 & 0.1683 & 0.0091 \\
\hline
\end{tabular}

ASTM C 1260 Armstrong Cement with Georgetown Aggregate

Charleroi Lock and Dam Project

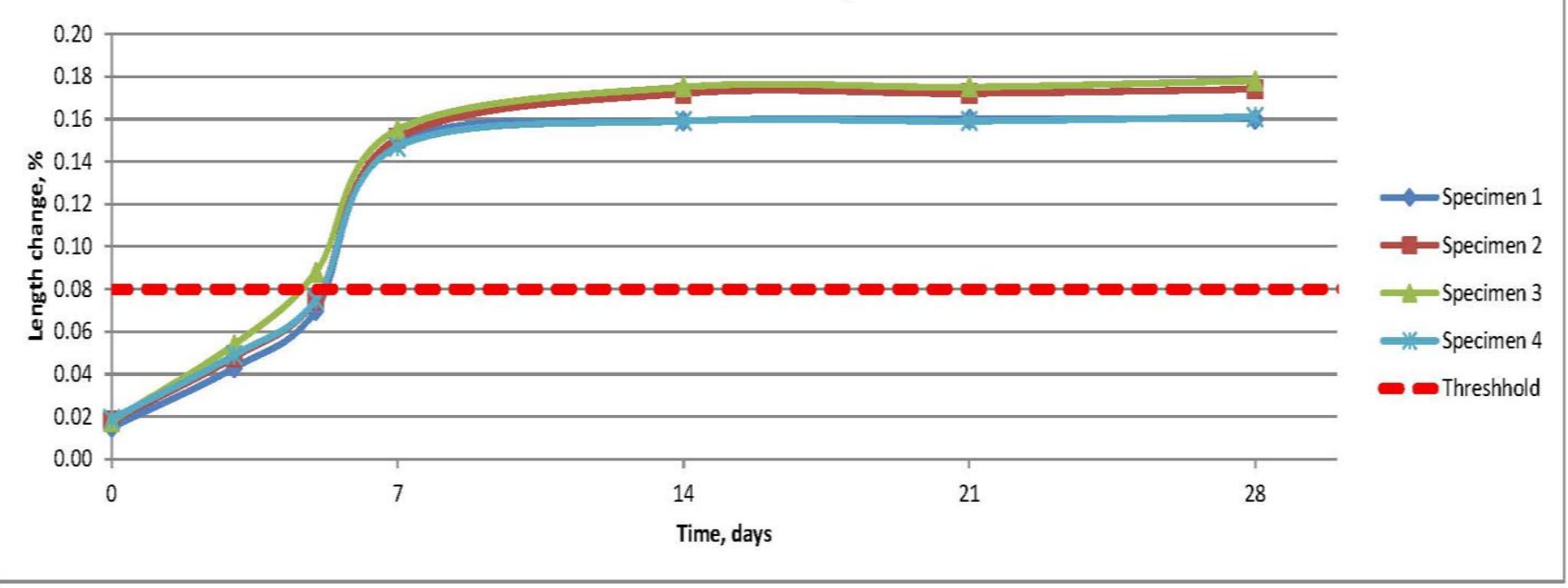




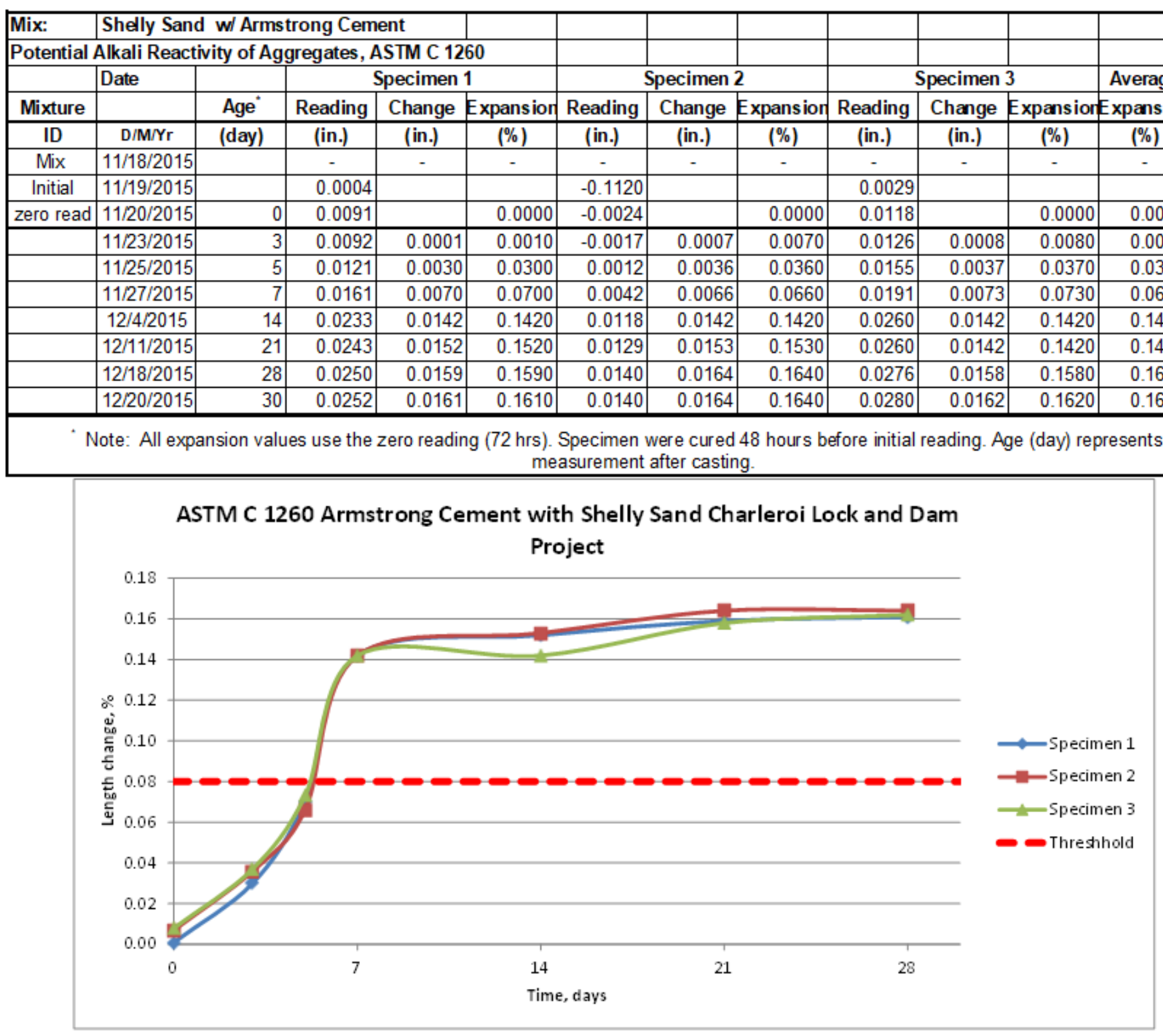




\section{Appendix C: Worksheets for ASTM C1567}




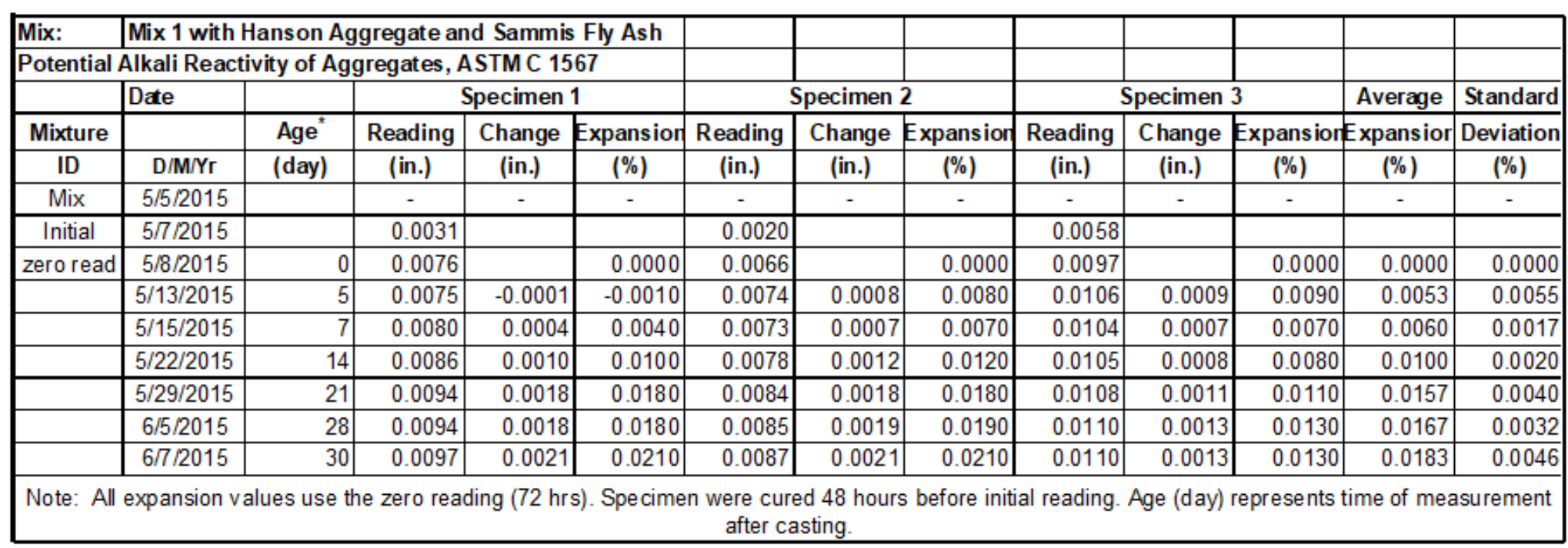

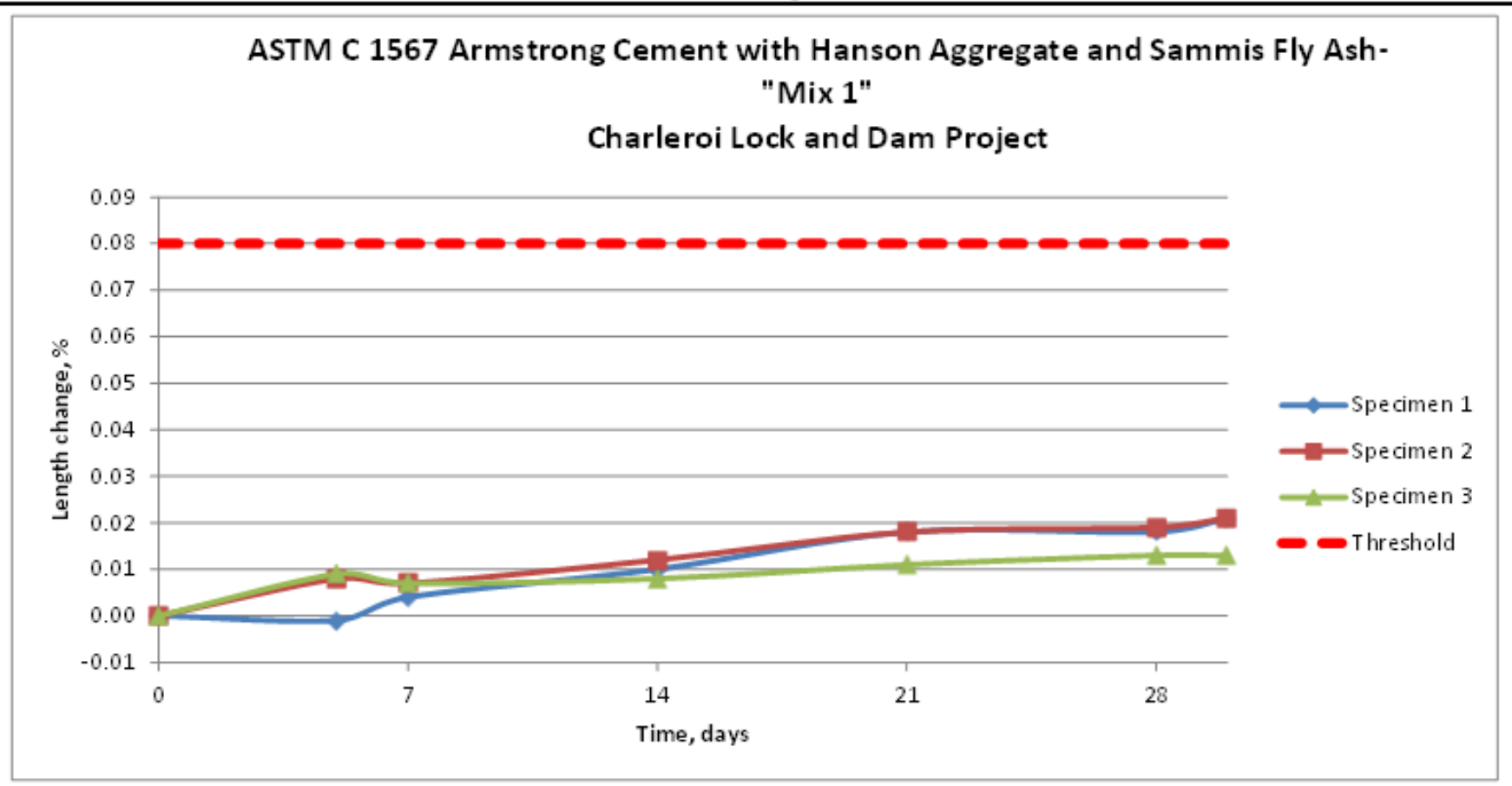




\begin{tabular}{|c|c|c|c|c|c|c|c|c|c|c|c|c|c|c|c|c|}
\hline \begin{tabular}{|l|} 
Mix: \\
\end{tabular} & \multicolumn{5}{|c|}{ Mix 1 with Hanson Aggregate and Ft. Martin Fly Ash } & & & & & & & & & & & \\
\hline \multicolumn{17}{|c|}{ Potential Alkali Reactivity of Aggregates, ASTM C 1567} \\
\hline & Date & & \multicolumn{3}{|c|}{ Specimen 1} & \multicolumn{3}{|c|}{ Specimen 2} & \multicolumn{3}{|c|}{ Specimen 3} & \multicolumn{3}{|c|}{ Specimen 4} & Average & Standard \\
\hline Mixture & & Age $^{*}$ & Reading & Change & Expansion & Reading & Change & Expansion & Reading & Change & Expansion & Reading & Change & Expansior & Expansion & Deviation \\
\hline ID & $\mathrm{D} / \mathrm{M} / \mathrm{Yr}$ & (day) & (in.) & (in.) & $(\%)$ & (in.) & (in.) & $(\%)$ & (in.) & (in.) & $(\%)$ & (in.) & (in.) & $(\%)$ & $(\%)$ & $(\%)$ \\
\hline Mix & $5 / 5 / 2015$ & & - & - & - & - & - & - & - & - & - & - & - & - & - & - \\
\hline Initial & $5 / 7 / 2015$ & & 0.0021 & & & \begin{tabular}{l|}
-0.0032 \\
\end{tabular} & & & 0.0069 & & & 0.0018 & & & & \\
\hline zero read & $5 / 8 / 2015$ & 0 & 0.0066 & & 0.0000 & 0.0017 & & 0.0000 & 0.0118 & & 0.0000 & 0.0053 & & 0.0000 & 0.0000 & 0.0000 \\
\hline & $5 / 13 / 2015$ & 5 & 0.0076 & 0.0010 & 0.0100 & 0.0019 & 0.0002 & 0.0020 & 0.0120 & 0.0002 & 0.0020 & 0.0063 & 0.0010 & 0.0100 & 0.0060 & 0.0046 \\
\hline & $5 / 15 / 2015$ & 7 & 0.0078 & 0.0012 & 0.0120 & 0.0027 & 0.0010 & 0.0100 & 0.0127 & 0.0009 & 0.0090 & 0.0066 & 0.0013 & 0.0130 & 0.0110 & 0.0018 \\
\hline & $5 / 22 / 2015$ & 14 & 0.0080 & 0.0014 & 0.0140 & 0.0026 & 0.0009 & 0.0090 & 0.0128 & 0.0010 & 0.0100 & 0.0067 & 0.0014 & 0.0140 & 0.0118 & 0.0026 \\
\hline & $5 / 29 / 2015$ & 21 & 0.0081 & 0.0015 & 0.0150 & 0.0030 & 0.0013 & 0.0130 & 0.0130 & 0.0012 & 0.0120 & 0.0070 & 0.0017 & 0.0170 & 0.0143 & 0.0022 \\
\hline & $6 / 5 / 2015$ & 28 & 0.0084 & 0.0018 & 0.0180 & 0.0032 & 0.0015 & 0.0150 & 0.0135 & 0.0017 & 0.0170 & 0.0075 & 0.0022 & 0.0220 & 0.0180 & 0.0029 \\
\hline & $6 / 7 / 2015$ & 30 & 0.0085 & 0.0019 & 0.0190 & 0.0034 & 0.0017 & 0.0170 & 0.0138 & 0.0020 & 0.0200 & 0.0076 & 0.0023 & 0.0230 & 0.0198 & 0.0025 \\
\hline
\end{tabular}

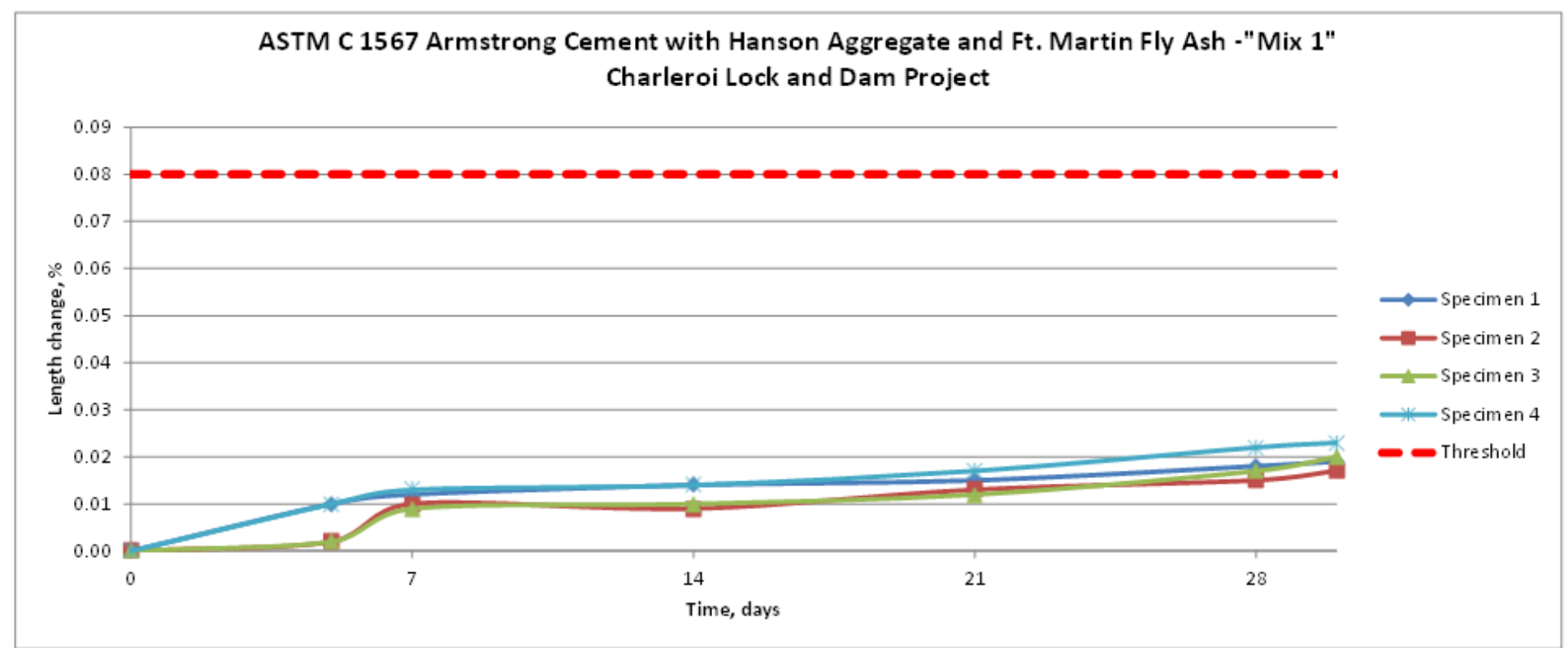




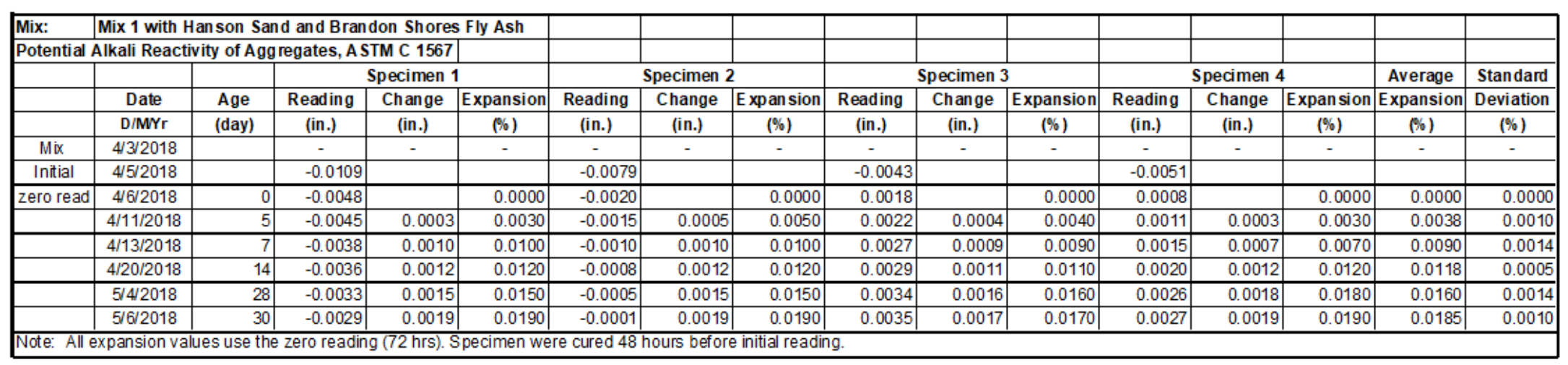

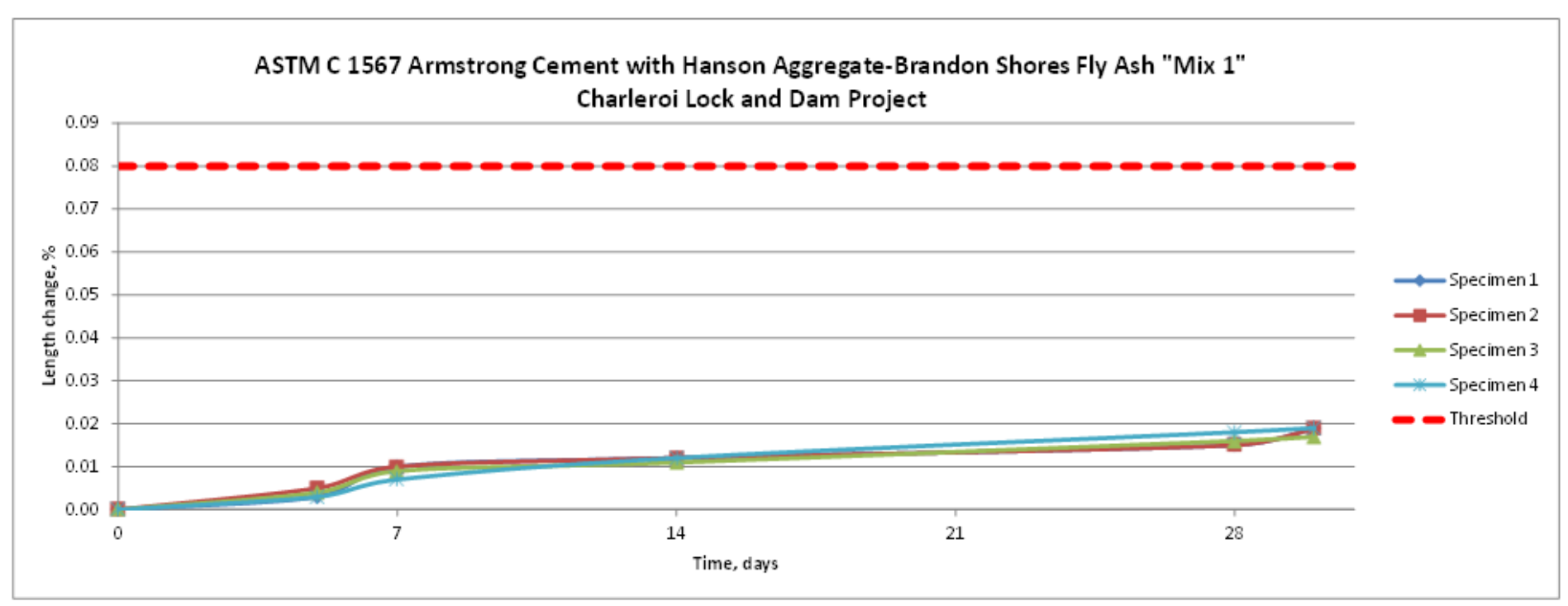




\begin{tabular}{|c|c|c|c|c|c|c|c|c|c|c|c|c|c|c|c|c|}
\hline \multirow{3}{*}{\begin{tabular}{|l|} 
Mix: \\
Potential \\
\end{tabular}} & \multicolumn{16}{|c|}{ Mix 1 with Georgetown Aggregate and Sammis Fly Ash } \\
\hline & \multicolumn{5}{|c|}{ Alkali Reactivity of Aggregates, ASTM C 1567} & & & & & & & & & & & \multirow[b]{2}{*}{ Standard } \\
\hline & Date & & \multicolumn{3}{|c|}{ Specimen 1} & \multicolumn{3}{|c|}{ Specimen 2} & \multicolumn{3}{|c|}{ Specimen 3} & \multicolumn{3}{|c|}{ Specimen 4} & Average & \\
\hline Mixture & & Age $^{*}$ & Reading & Change & Expansion & Reading & Change & Expansion & Reading & Change & Expansion & Reading & Change & \multicolumn{2}{|c|}{ ExpansiorExpansion } & Deviation \\
\hline ID & $\mathrm{D} / \mathrm{M} / \mathrm{Yr}$ & (day) & (in.) & (in.) & $(\%)$ & (in.) & (in.) & $(\%)$ & (in.) & (in.) & $(\%)$ & (in.) & (in.) & $(\%)$ & $(\%)$ & $(\%)$ \\
\hline Mix & $5 / 5 / 2015$ & & - & - & - & - & - & - & - & - & - & - & - & - & - & - \\
\hline Initial & $5 / 7 / 2015$ & & -0.0002 & & & -0.0057 & & & -0.0071 & & & -0.0016 & & & & \\
\hline \multirow[t]{7}{*}{ zero read } & $5 / 8 / 2015$ & 0 & 0.0060 & & 0.0000 & 0.0005 & & 0.0000 & -0.0010 & & 0.0000 & 0.0047 & & 0.0000 & 0.0000 & 0.0000 \\
\hline & $5 / 13 / 2015$ & 5 & 0.0064 & 0.0004 & 0.0040 & 0.0008 & 0.0003 & 0.0030 & -0.0010 & 0.0000 & 0.0000 & 0.0048 & 0.0001 & 0.0010 & 0.0020 & 0.0018 \\
\hline & $5 / 15 / 2015$ & 7 & 0.0065 & 0.0005 & 0.0050 & 0.0010 & 0.0005 & 0.0050 & -0.0006 & 0.0004 & 0.0040 & 0.0048 & 0.0001 & 0.0010 & 0.0038 & $0.001 \mathrm{~s}$ \\
\hline & $5 / 22 / 2015$ & 14 & 0.0066 & 0.0006 & 0.0060 & 0.0010 & 0.0005 & 0.0050 & -0.0005 & 0.0005 & 0.0050 & 0.0050 & 0.0003 & 0.0030 & 0.0048 & 0.0013 \\
\hline & $5 / 29 / 2015$ & 21 & 0.0066 & 0.0006 & 0.0060 & 0.0010 & 0.0005 & 0.0050 & -0.0005 & 0.0005 & 0.0050 & 0.0051 & 0.0004 & 0.0040 & 0.0050 & 0.0008 \\
\hline & $6 / 5 / 2015$ & 28 & 0.0066 & 0.0006 & 0.0060 & 0.0015 & 0.0010 & 0.0100 & -0.0004 & 0.0006 & 0.0060 & 0.0055 & 0.0008 & 0.0080 & 0.0075 & 0.0019 \\
\hline & $6 / 7 / 2015$ & 30 & 0.0067 & 0.0007 & 0.0070 & 0.0014 & 0.0009 & 0.0090 & -0.0004 & 0.0006 & 0.0060 & 0.0055 & 0.0008 & 0.0080 & 0.0075 & 0.0013 \\
\hline
\end{tabular}

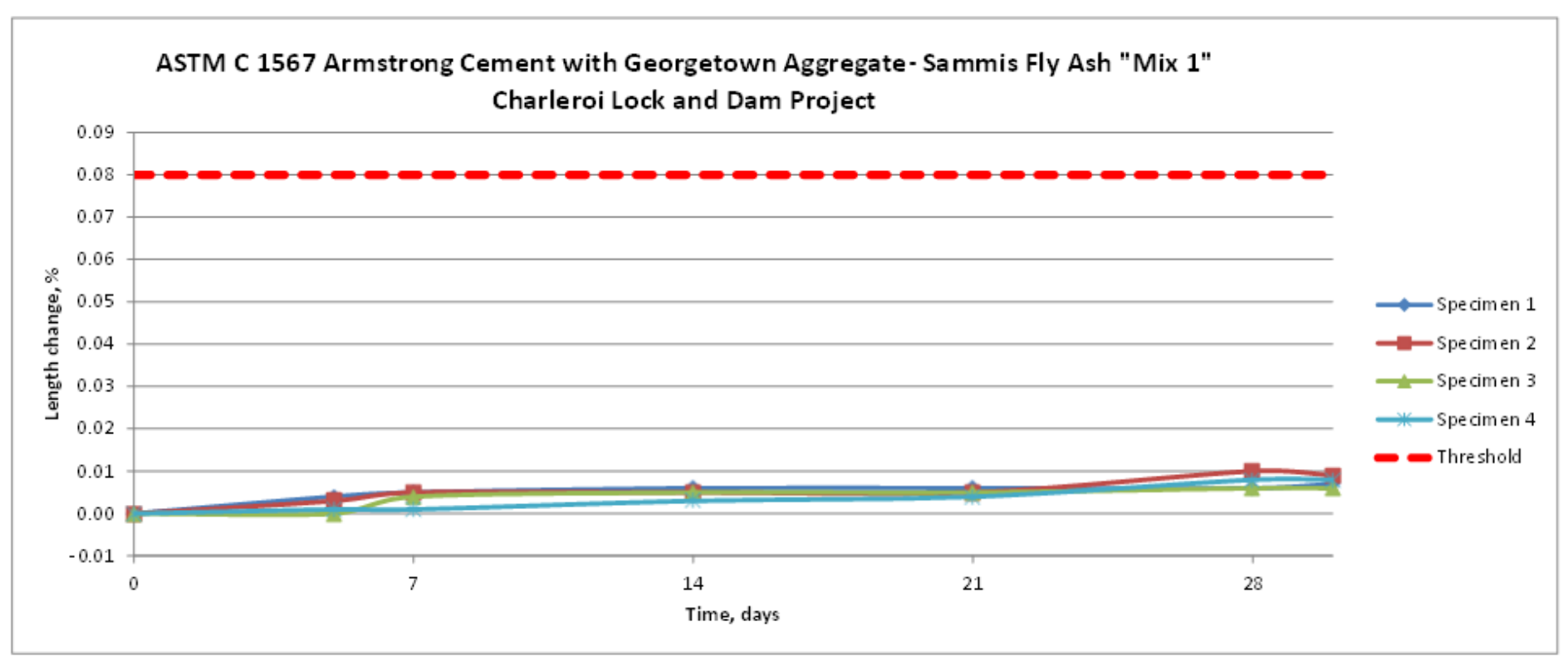




\begin{tabular}{|c|c|c|c|c|c|c|c|c|c|c|c|c|c|c|c|c|}
\hline \multirow{4}{*}{\begin{tabular}{|l} 
Mix: \\
Potential \\
\\
Mixture \\
\end{tabular}} & \multicolumn{16}{|c|}{ Mix 1 with Georgetown Aggregate and Ft. Martin Fly Ash } \\
\hline & \multicolumn{5}{|c|}{ Alkali Reactivity of Aggregates, A STM C 1567} & & & & & & & & & & & \multirow[b]{2}{*}{ Standard } \\
\hline & \multirow[t]{2}{*}{ Date } & \multirow[b]{2}{*}{$\operatorname{Age}^{*}$} & \multicolumn{3}{|c|}{ Specimen 1} & \multicolumn{3}{|c|}{ Specimen 2} & \multicolumn{3}{|c|}{ Specimen 3} & \multicolumn{3}{|c|}{ Specimen 4} & Average & \\
\hline & & & Reading & Change & Expansion & Reading & Change & Expansior & Reading & Change & Expansion & Reading & Change & Expans ion & Expansion & Deviation \\
\hline ID & $\mathrm{D} / \mathrm{M} / \mathrm{Yr}$ & (day) & (in.) & (in.) & $(\%)$ & (in.) & (in.) & $(\%)$ & (in.) & (in.) & $(\%)$ & (in.) & (in.) & $(\%)$ & $(\%)$ & $(\%)$ \\
\hline Mix & $5 / 5 / 2015$ & & - & - & - & - & - & - & - & - & - & - & - & - & - & - \\
\hline Initial & $5 / 7 / 2015$ & & -0.0160 & & & 0.0050 & & & -0.0088 & & & 0.0016 & & & & \\
\hline zero read & $5 / 8 / 2015$ & 0 & -0.0102 & & 0.0000 & 0.0105 & & 0.0000 & 0.0020 & & 0.0000 & 0.0080 & & 0.0000 & 0.0000 & 0.0000 \\
\hline & $5 / 13 / 2015$ & 5 & -0.0100 & 0.0002 & 0.0020 & 0.0106 & 0.0001 & 0.0010 & 0.0020 & 0.0000 & 0.0000 & 0.0080 & 0.0000 & 0.0000 & 0.0008 & 0.0010 \\
\hline & 5/15/2015 & 7 & -0.0099 & 0.0003 & 0.0030 & 0.0109 & 0.0004 & 0.0040 & 0.0022 & 0.0002 & 0.0020 & 0.0081 & 0.0001 & 0.0010 & 0.0025 & 0.0013 \\
\hline & $5 / 22 / 2015$ & 14 & -0.0097 & 0.0005 & 0.0050 & 0.0110 & 0.0005 & 0.0050 & 0.0024 & 0.0004 & 0.0040 & 0.0082 & 0.0002 & 0.0020 & 0.0040 & 0.0014 \\
\hline & $5 / 29 / 2015$ & 21 & -0.0096 & 0.0006 & 0.0060 & 0.0113 & 0.0008 & 0.0080 & 0.0025 & 0.0005 & 0.0050 & 0.0085 & 0.0005 & 0.0050 & 0.0060 & 0.0014 \\
\hline & $6 / 5 / 2015$ & 28 & \begin{tabular}{|c|}
-0.0096 \\
\end{tabular} & 0.0006 & 0.0060 & 0.0114 & 0.0009 & 0.0090 & 0.0026 & 0.0006 & 0.0060 & 0.0086 & 0.0006 & 0.0060 & 0.0068 & 0.0015 \\
\hline & $6 / 7 / 2015$ & 30 & -0.0096 & 0.0006 & 0.0060 & 0.0115 & 0.0010 & 0.0100 & 0.0028 & 0.0008 & 0.0080 & 0.0086 & 0.0006 & 0.0060 & 0.0075 & 0.0019 \\
\hline
\end{tabular}

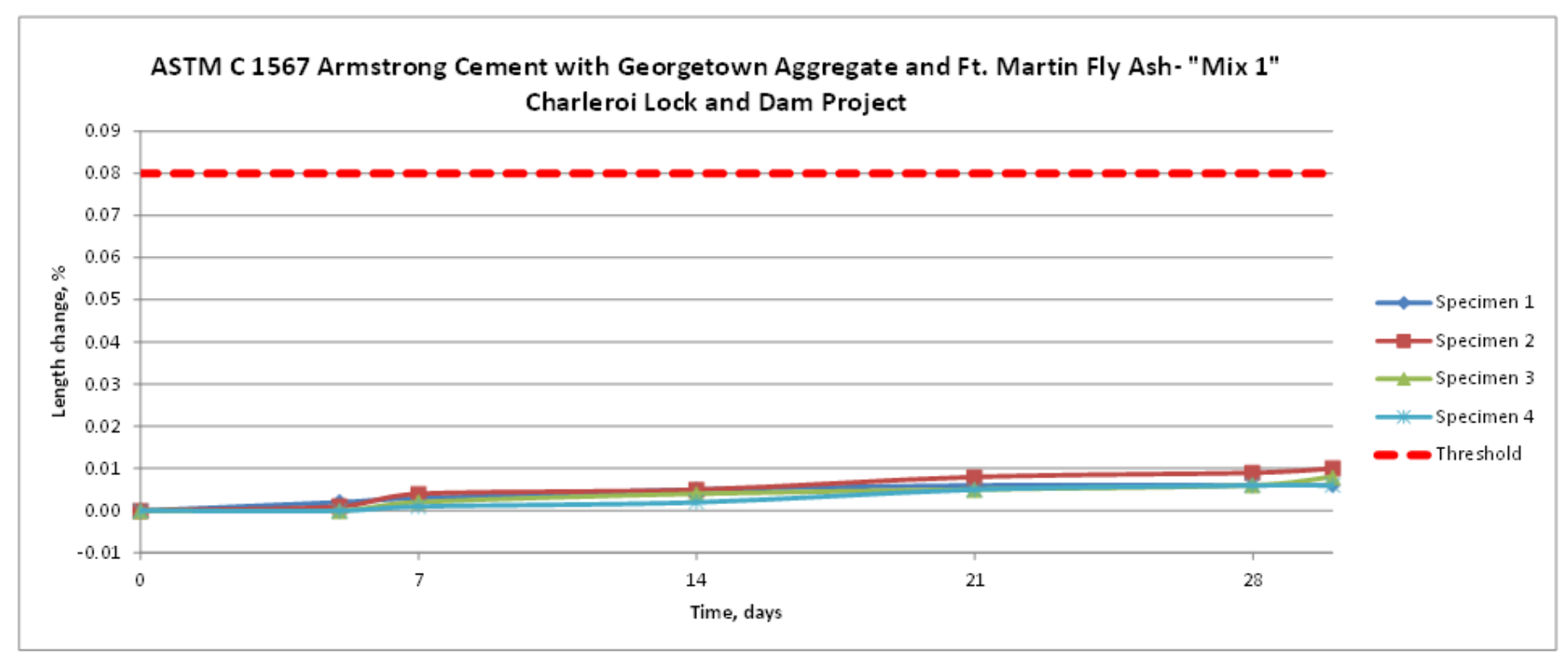




\begin{tabular}{|c|c|c|c|c|c|c|c|c|c|c|c|c|c|}
\hline Mix ID: & Mix 1 with $\mathrm{Ge}$ & getown Sal & nd and Lon & view Fly A & & & & & & & & & \\
\hline Potential & Alkali Reactivi & of Aggrega & ates, ASTM & 1567 & & & & & & & & & \\
\hline & & & & pecimen 1 & & & Specimen 2 & & & Specimen 3 & & Average & Standard \\
\hline & Date & Age & Reading & Change & Expansion & Reading & Change & Expansion & Reading & Change & Expansion & Expansion & Deviation \\
\hline Mix & $11 / 14 / 2017$ & & - & - & - & - & - & - & - & - & - & - & - \\
\hline Initial & $11 / 16 / 2017$ & & -0.0091 & & & -0.0127 & & & -0.0086 & & & & \\
\hline \begin{tabular}{|l|} 
zero read \\
\end{tabular} & $11 / 17 / 2017$ & 0 & -0.0025 & & 0.0000 & -0.0058 & & 0.0000 & -0.0015 & & 0.0000 & 0.0000 & 0.0000 \\
\hline & $11 / 22 / 2017$ & 5 & -0.0021 & 0.0004 & 0.0040 & -0.0058 & 0.0000 & 0.0000 & -0.0012 & 0.0003 & 0.0030 & 0.0023 & 0.0021 \\
\hline & $11 / 24 / 2017$ & 7 & -0.0021 & 0.0004 & 0.0040 & -0.0054 & 0.0004 & 0.0040 & -0.0011 & 0.0004 & 0.0040 & \begin{tabular}{l|l}
0.0040 \\
\end{tabular} & 0.0000 \\
\hline & $12 / 1 / 2017$ & 14 & -0.0019 & 0.0006 & 0.0060 & -0.0052 & 0.0006 & 0.0060 & -0.0009 & 0.0006 & 0.0060 & 0.0060 & 0.0000 \\
\hline & $12 / 8 / 2017$ & 21 & -0.0019 & 0.0006 & 0.0060 & -0.0051 & 0.0007 & 0.0070 & -0.0006 & 0.0009 & 0.0090 & 0.0073 & 0.0015 \\
\hline & $12 / 15 / 2017$ & 28 & -0.0017 & 0.0008 & 0.0080 & -0.0050 & 0.0008 & 0.0080 & -0.0006 & 0.0009 & 0.0090 & 0.0083 & 0.0006 \\
\hline & $12 / 17 / 2017$ & 30 & -0.0016 & 0.0009 & 0.0090 & -0.0049 & 0.0009 & 0.0090 & -0.0005 & 0.0010 & 0.0100 & \begin{tabular}{|c|}
0.0093 \\
\end{tabular} & 0.0006 \\
\hline
\end{tabular}

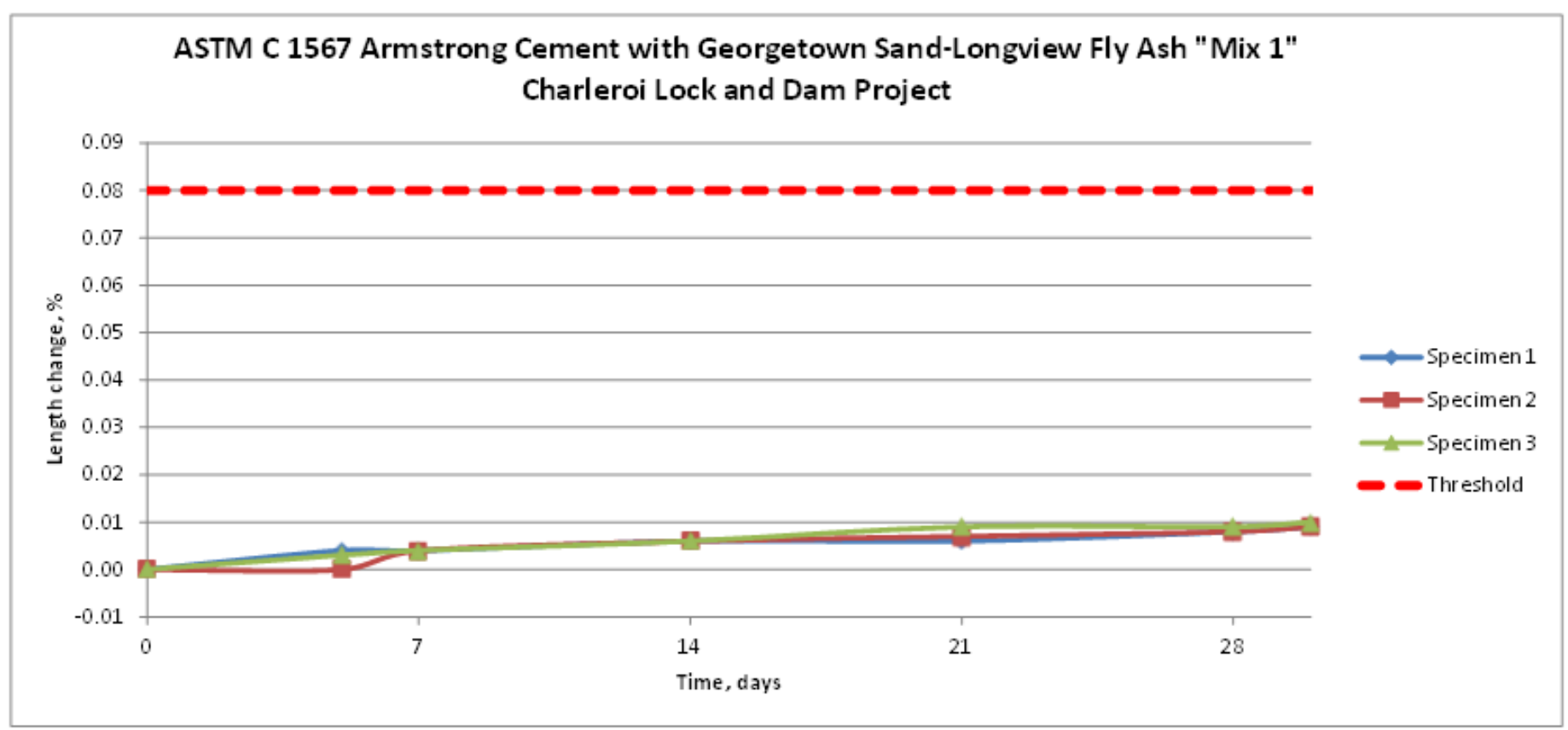




\begin{tabular}{|c|c|c|c|c|c|c|c|c|c|c|c|c|c|c|c|c|}
\hline Mix: & \multicolumn{16}{|c|}{ Mix 1 with Georgetown Sand and Brandon Shores Fly Ash } \\
\hline \multicolumn{17}{|c|}{ Potential Alkali Reactivity of Aggregates, ASTM C 1567 } \\
\hline & & & \multicolumn{3}{|c|}{ Specimen 1} & \multicolumn{3}{|c|}{ Specimen 2} & \multicolumn{3}{|c|}{ Specimen 3} & \multicolumn{3}{|c|}{ Specimen 4} & Average & Standard \\
\hline & Date & Age & Reading & Change & Expansion & Reading & Change & Expansion & Reading & Change & Expansion & Reading & Change & Expansion & Expansion & Deviation \\
\hline & $\mathrm{D} / \mathrm{M} Y \mathrm{r}$ & (day) & (in.) & (in.) & $\%)$ & (in.) & (in.) & $(\%)$ & (in.) & (in.) & $(\%)$ & (in.) & (in.) & $(\%)$ & $\%$ & (\%) \\
\hline Initial & $4 / 5 / 2018$ & & -0.0124 & & & -0.0086 & & & -0.0092 & & & -0.0103 & & & & \\
\hline zero read & $4 / 6 / 2018$ & 0 & -0.0061 & & 0.0000 & -0.0016 & & 0.0000 & -0.0023 & & 0.0000 & -0.0032 & & 0.0000 & 0.0000 & 0.0000 \\
\hline & 4/11/2018 & 5 & -0.0060 & 0.0001 & 0.0010 & -0.0012 & 0.0004 & 0.0040 & -0.0021 & 0.0002 & 0.0020 & -0.0030 & 0.0002 & 0.0020 & 0.0023 & 0.0013 \\
\hline & $4 / 13 / 2018$ & 7 & -0.0058 & 0.0003 & 0.0030 & -0.0010 & 0.0006 & 0.0060 & -0.0020 & 0.0003 & 0.0030 & -0.0028 & 0.0004 & 0.0040 & 0.0040 & 0.0014 \\
\hline & $5 / 4 / 2018$ & 28 & -0.0053 & 0.0008 & \begin{tabular}{l|l|}
0.0080 \\
\end{tabular} & -0.0007 & 0.0009 & 0.0090 & -0.0012 & 0.0011 & 0.0110 & -0.0025 & 0.0007 & 0.0070 & 0.0088 & 0.0017 \\
\hline & $5 / 6 / 2018$ & 30 & -0.0052 & 0.0009 & 0.0090 & -0.0007 & 0.0009 & 0.0090 & -0.0013 & 0.0010 & 0.0100 & -0.0024 & 0.0008 & 0.0080 & 0.0090 & 0.0008 \\
\hline
\end{tabular}

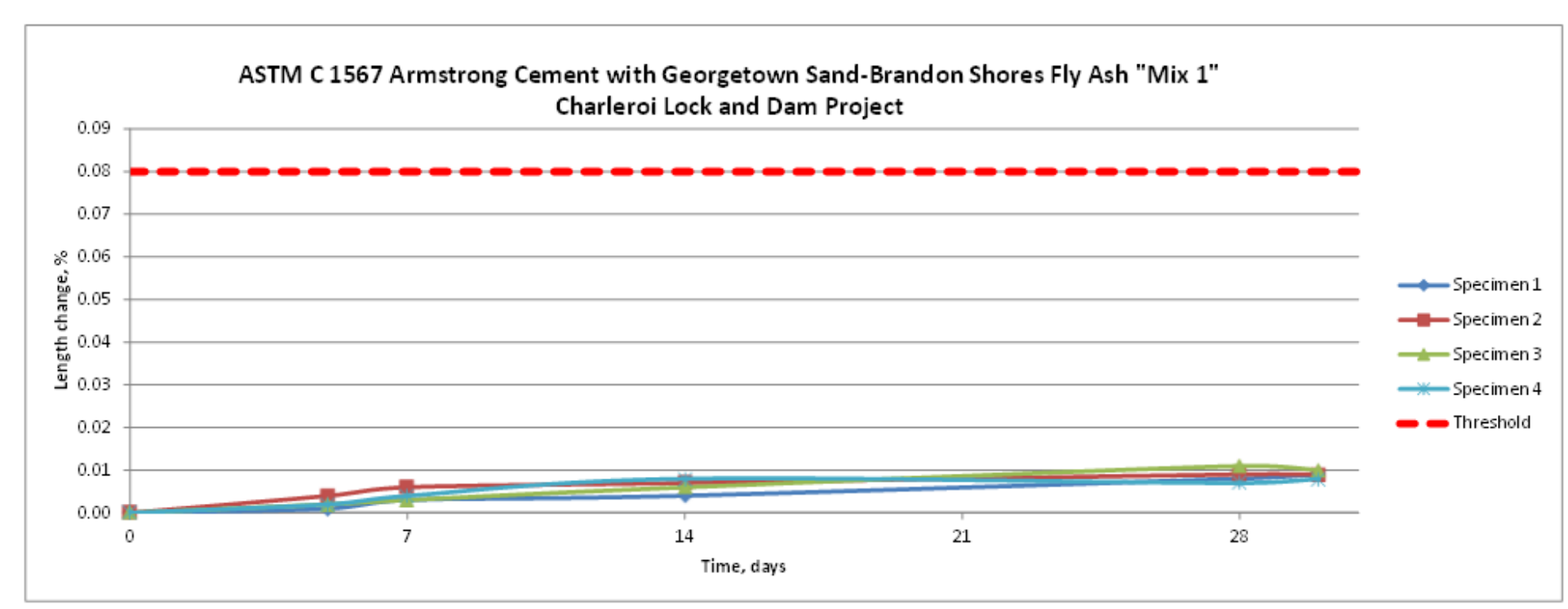




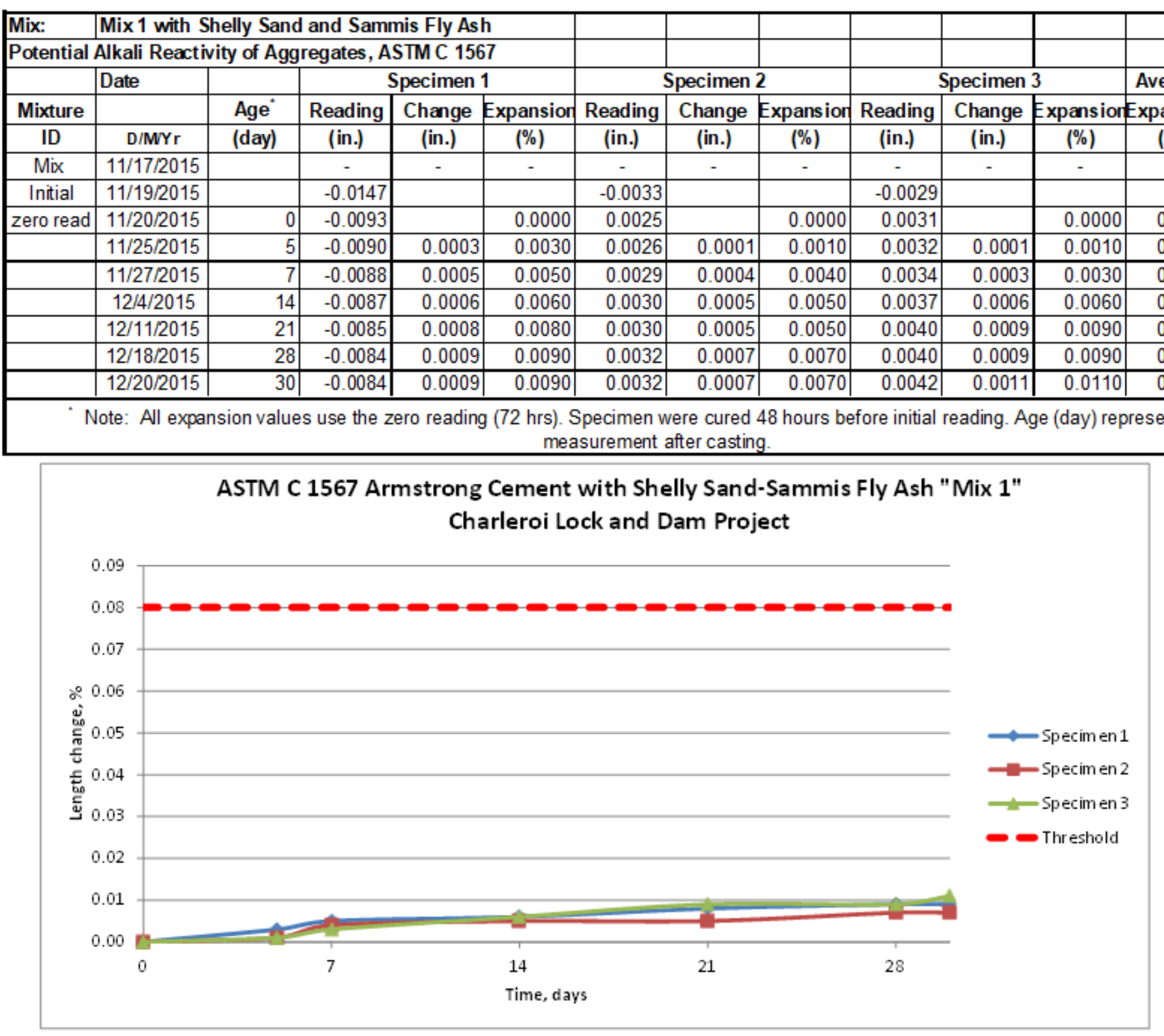




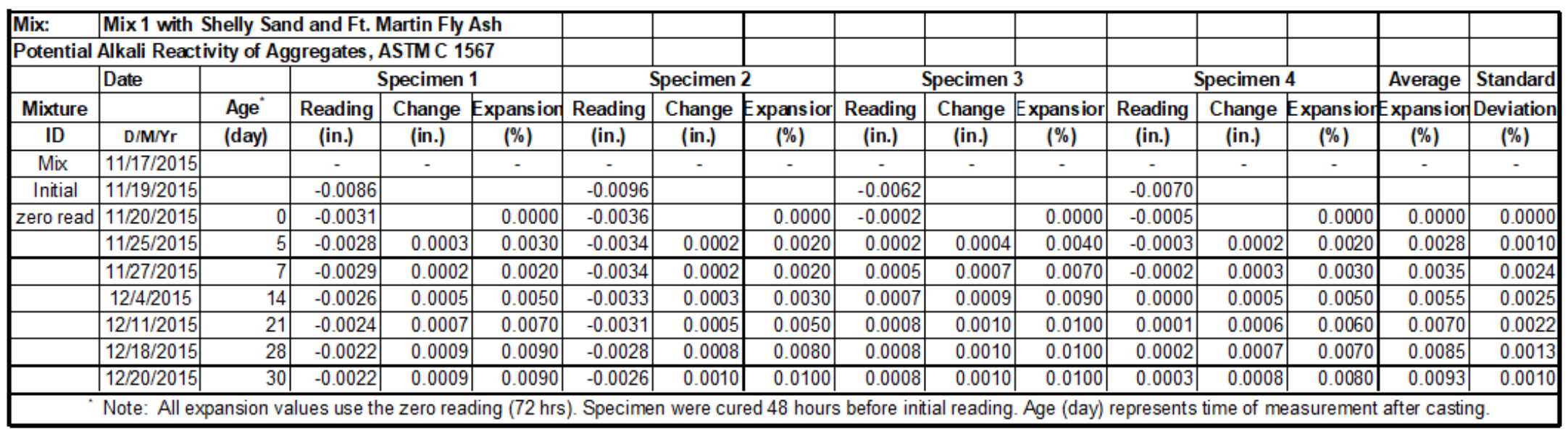

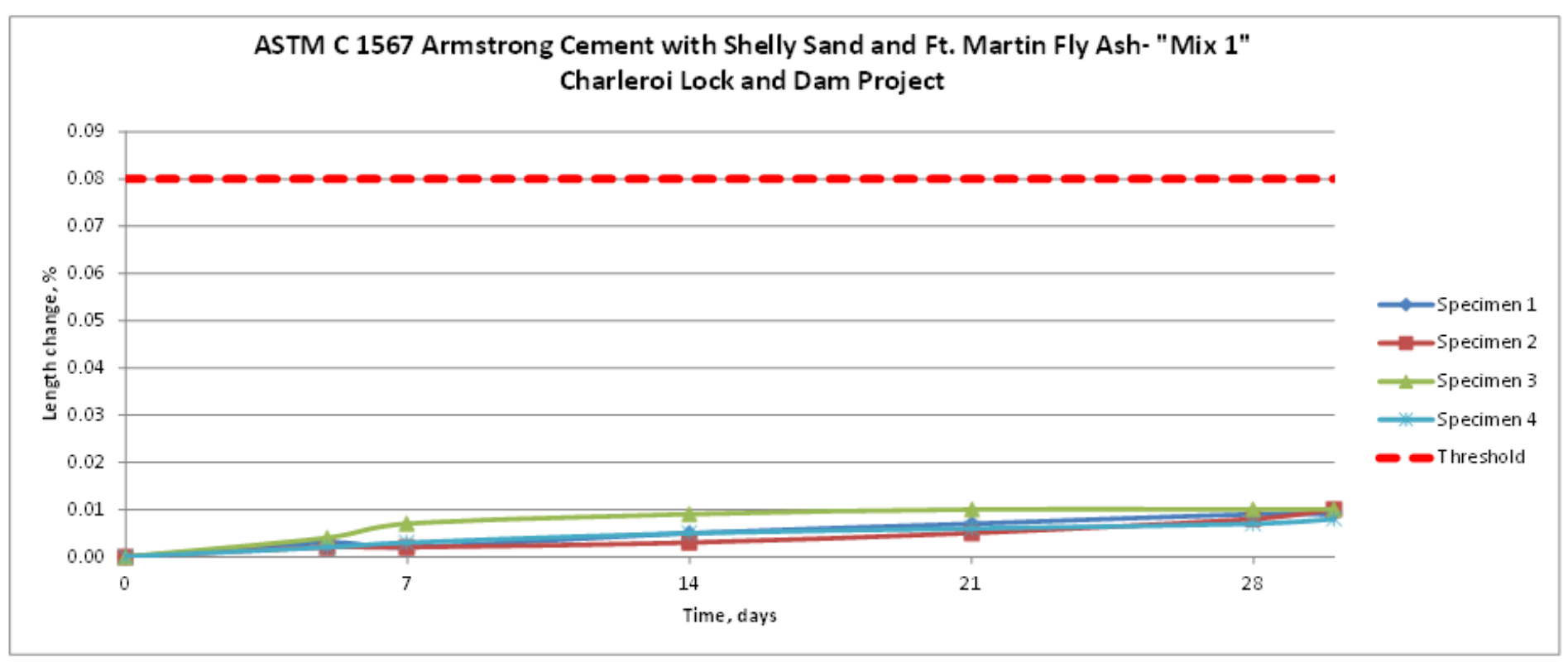




\begin{tabular}{|c|c|c|c|c|c|c|c|c|c|c|c|c|c|}
\hline Mix: & Mix 1 with S & elley Sand & and Longv & ewFly Ash & & & & & & & & & \\
\hline Potential & Alkali Reacti & $y$ of Aggre & gates, AST & AC 1567 & & & & & & & & & \\
\hline & & & & pecimen 1 & & & Specimen 2 & & & Specimen 3 & & Average & Standard \\
\hline & Date & Age & Reading & Change & Expansion & Reading & Change & Expansion & Reading & Change & Expansion & Expansion & Deviation \\
\hline & $\mathrm{D} / \mathrm{M} Y \mathrm{r}$ & (day) & (in.) & (in.) & $(\%)$ & (in.) & (in.) & $(\%)$ & (in.) & (in.) & $(\%)$ & $(\%)$ & $(\%)$ \\
\hline Mix & $11 / 14 / 2017$ & & - & - & - & - & - & - & - & - & - & - & - \\
\hline Initial & $11 / 16 / 2017$ & & -0.0103 & & & -0.0122 & & & -0.0054 & & & & \\
\hline zero read & $11 / 17 / 2017$ & 0 & -0.0031 & & 0.0000 & -0.0050 & & 0.0000 & 0.0016 & & 0.0000 & 0.0000 & 0.0000 \\
\hline & $11 / 22 / 2017$ & 5 & -0.0030 & 0.0001 & 0.0010 & -0.0048 & 0.0002 & 0.0020 & 0.0017 & 0.0001 & 0.0010 & 0.0013 & 0.0006 \\
\hline & $11 / 24 / 2017$ & 7 & -0.0029 & 0.0002 & 0.0020 & -0.0048 & 0.0002 & 0.0020 & 0.0019 & 0.0003 & 0.0030 & 0.0023 & 0.0006 \\
\hline & $12 / 1 / 2017$ & 14 & -0.0028 & 0.0003 & 0.0030 & -0.0044 & 0.0006 & 0.0060 & 0.0020 & 0.0004 & 0.0040 & 0.0043 & 0.0015 \\
\hline & $12 / 8 / 2017$ & 21 & -0.0027 & 0.0004 & 0.0040 & -0.0046 & 0.0004 & 0.0040 & 0.0022 & 0.0006 & 0.0060 & 0.0047 & 0.0012 \\
\hline & $12 / 15 / 2017$ & 28 & -0.0027 & 0.0004 & 0.0040 & -0.0044 & 0.0006 & 0.0060 & 0.0025 & 0.0009 & 0.0090 & 0.0063 & 0.0025 \\
\hline & $12 / 17 / 2017$ & 30 & -0.0025 & 0.0006 & 0.0060 & -0.0042 & 0.0008 & 0.0080 & 0.0024 & 0.0008 & 0.0080 & 0.0073 & 0.0012 \\
\hline
\end{tabular}

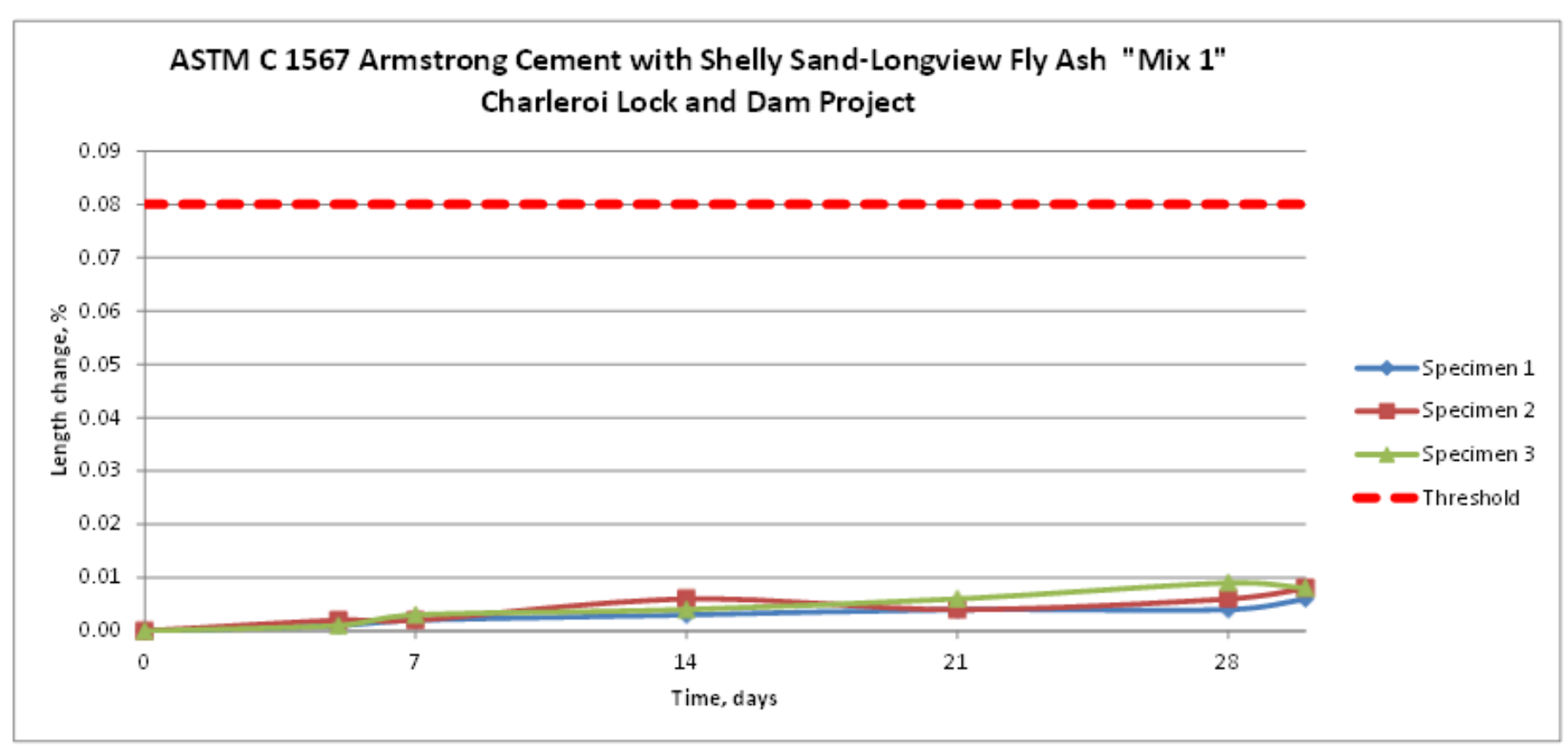




\begin{tabular}{|c|c|c|c|c|c|c|c|c|c|c|c|c|c|c|c|c|}
\hline Mix: & \multicolumn{5}{|c|}{ Mix 1 with Shelley Sand and Brandon Shores Fly Ash } & & & & & & & & & & & \\
\hline \multicolumn{17}{|c|}{\begin{tabular}{|l|l|} 
Potential Alkali Reactivity of Agg regates, A STM C 1567 & \\
\end{tabular}} \\
\hline & & & \multicolumn{3}{|c|}{ Specimen 1} & \multicolumn{3}{|c|}{ Specimen 2} & \multicolumn{3}{|c|}{ Specimen 3} & \multicolumn{3}{|c|}{ Specimen 4} & Average & \begin{tabular}{|l} 
Standard \\
\end{tabular} \\
\hline & Date & Age & Reading & Change & Expansion & Reading & Change & Expansion & \begin{tabular}{|l|} 
Reading \\
\end{tabular} & Change & Expansion & \begin{tabular}{|l|} 
Reading \\
\end{tabular} & Change & Expansion & Expansion & \begin{tabular}{|l|} 
Deviation \\
\end{tabular} \\
\hline & $\mathrm{D} / \mathrm{M} Y \mathrm{r}$ & (day) & (in.) & (in.) & $\%$ & (in.) & (in.) & $(\%)$ & (in.) & (in.) & $(\%)$ & (in.) & (in.) & $(\%)$ & $\%)$ & $(\%)$ \\
\hline Initial & $4 / 5 / 2018$ & & -0.0090 & & & -0.0109 & & & -0.0090 & & & -0.0098 & & & & \\
\hline zero read & $4 / 6 / 2018$ & 0 & \begin{tabular}{|c|}
-0.0017 \\
\end{tabular} & & 0.0000 & $\mid-0.0036$ & & 0.0000 & \begin{tabular}{|c|}
-0.0017 \\
\end{tabular} & & 0.0000 & -0.0023 & & 0.0000 & 0.0000 & 0.0000 \\
\hline & $4 / 11 / 2018$ & 5 & -0.0016 & 0.0001 & 0.0010 & -0.0035 & 0.0001 & 0.0010 & -0.0015 & 0.0002 & 0.0020 & -0.0022 & 0.0001 & 0.0010 & 0.0013 & 0.0005 \\
\hline & $4 / 13 / 2018$ & 7 & $\mid-0.0014$ & 0.0003 & 0.0030 & -0.0033 & 0.0003 & 0.0030 & -0.0012 & 0.0005 & 0.0050 & -0.0018 & 0.0005 & 0.0050 & 0.0040 & 0.0012 \\
\hline & $5 / 4 / 2018$ & 28 & -0.0010 & 0.0007 & \begin{tabular}{l|l}
0.0070 \\
\end{tabular} & -0.0027 & 0.0009 & 0.0090 & -0.0009 & 0.0008 & 0.0080 & -0.0017 & 0.0006 & 0.0060 & 0.0075 & 0.0013 \\
\hline & $5 / 6 / 2018$ & 30 & $\mid-0.0009$ & 0.0008 & 0.0080 & -0.0029 & 0.0007 & 0.0070 & -0.0009 & 0.0008 & 0.0080 & -0.0016 & 0.0007 & 0.0070 & 0.0075 & 0.0006 \\
\hline
\end{tabular}

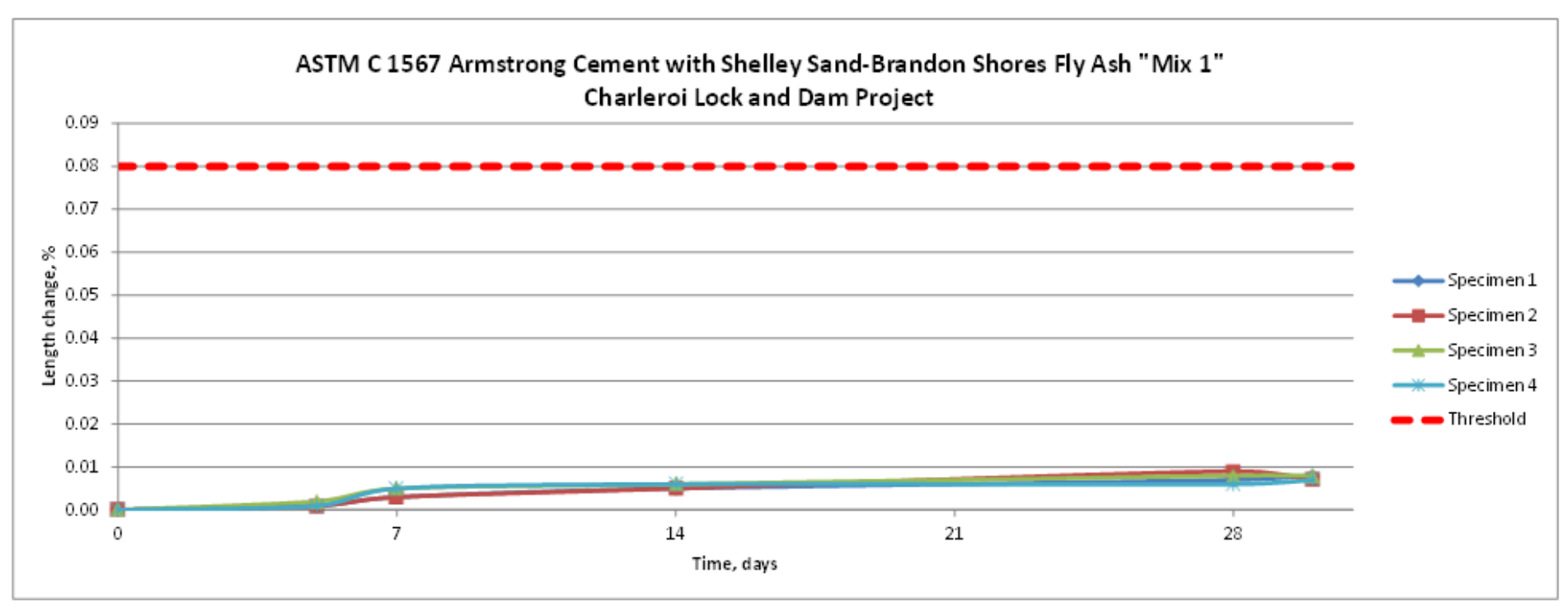




\begin{tabular}{|c|c|c|c|c|c|c|c|c|c|c|c|c|c|}
\hline Mix: & \multicolumn{13}{|c|}{ Mix 1 with Hans on Aggregate and Longview Fly Ash } \\
\hline \multicolumn{14}{|c|}{ Potential Alkali Reactivity of Aggregates, ASTM C 1567} \\
\hline & & & \multicolumn{3}{|c|}{ Specimen 1} & \multicolumn{3}{|c|}{ Specimen 2} & \multicolumn{3}{|c|}{ Specimen 3} & Average & Standard \\
\hline & Date & Age & Reading & Change & Expansion & \begin{tabular}{|l|} 
Reading \\
\end{tabular} & Change & Expansior & Reading & Change & Expansior & Expansion & Deviation \\
\hline & $\mathrm{D} / \mathrm{M} / \mathrm{Yr}$ & (day) & (in.) & (in.) & $(\%)$ & (in.) & (in.) & $(\%)$ & (in.) & (in.) & $(\%)$ & $(\%)$ & $(\%)$ \\
\hline Mix & $11 / 14 / 2017$ & & - & - & - & - & - & - & - & - & - & - & - \\
\hline Initial & $11 / 16 / 2017$ & & 0.0024 & & & -0.0135 & & & -0.0037 & & & & \\
\hline \multirow[t]{7}{*}{ zero read } & $11 / 17 / 2017$ & 0 & 0.0073 & & 0.0000 & -0.0078 & & 0.0000 & -0.0284 & & 0.0000 & 0.0000 & 0.0000 \\
\hline & $11 / 22 / 2017$ & 5 & 0.0081 & 0.0008 & 0.0080 & -0.0076 & 0.0002 & 0.0020 & -0.0280 & 0.0004 & 0.0040 & 0.0047 & 0.0034 \\
\hline & $11 / 24 / 2017$ & 7 & 0.0085 & 0.0012 & 0.0120 & -0.0074 & 0.0004 & \begin{tabular}{l|l|}
0.0040 \\
\end{tabular} & -0.0278 & 0.0006 & 0.0060 & 0.0073 & 0.0050 \\
\hline & $12 / 1 / 2017$ & 14 & 0.0089 & 0.0016 & 0.0160 & -0.0070 & 0.0008 & 0.0080 & -0.0276 & 0.0008 & 0.0080 & 0.0107 & 0.0065 \\
\hline & $12 / 8 / 2017$ & 21 & 0.0095 & 0.0022 & 0.0220 & -0.0068 & 0.0010 & $\begin{array}{ll}0.0100 \\
\end{array}$ & -0.0265 & 0.0019 & 0.0190 & 0.0170 & 0.0099 \\
\hline & $12 / 15 / 2017$ & 28 & 0.0096 & 0.0023 & 0.0230 & -0.0062 & 0.0016 & 0.0160 & -0.0268 & 0.0016 & 0.0160 & 0.0183 & 0.0097 \\
\hline & $12 / 17 / 2017$ & 30 & 0.0097 & 0.0024 & 0.0240 & -0.0060 & 0.0018 & 0.0180 & -0.0266 & 0.0018 & 0.0180 & 0.0200 & 0.0104 \\
\hline
\end{tabular}

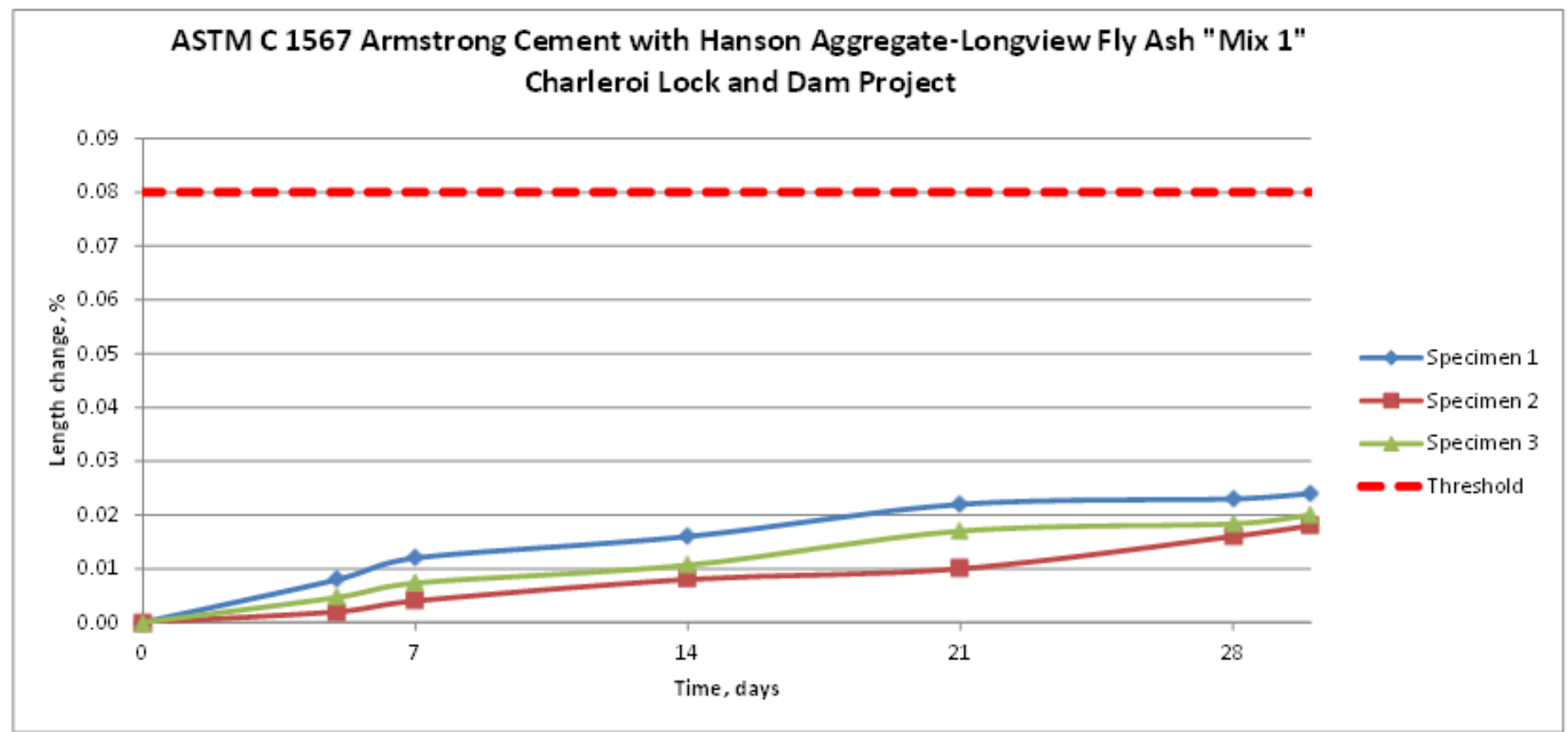




\begin{tabular}{|c|c|c|c|c|c|c|c|c|c|c|c|c|c|c|c|c|}
\hline Mix: & \multicolumn{5}{|c|}{ Mix 5 with Hanson Aggregate and Sammis Fly Ash } & & & & & & & & & & & \\
\hline \multicolumn{17}{|c|}{ Potential Alkali Reactivity of Aggregates, ASTM C 1567} \\
\hline & Date & & \multicolumn{3}{|c|}{ Specimen 1} & \multicolumn{3}{|c|}{ Specimen 2} & \multicolumn{3}{|c|}{ Specimen 3} & \multicolumn{3}{|c|}{ Specimen 4} & Average & Standard \\
\hline Mixture & & Age $^{*}$ & Reading & Change & Expansion & Reading & Change & Expansion & Reading & Change & Expans ion & Reading & Change & Expans ion & Expans ior & Deviation \\
\hline ID & $\mathrm{D} / \mathrm{M} / \mathrm{Yr}$ & (day) & (in.) & (in.) & $(\%)$ & (in.) & (in.) & $(\%)$ & (in.) & (in.) & $(\%)$ & (in.) & (in.) & $(\%)$ & $(\%)$ & $(\%)$ \\
\hline Mix & $5 / 5 / 2015$ & & - & - & - & - & - & - & - & - & - & - & - & - & - & - \\
\hline Initial & $5 / 7 / 2015$ & & 0.0023 & & & 0.0018 & & & 0.0059 & & & -0.0236 & & & & \\
\hline zero read & $5 / 8 / 2015$ & 0 & 0.0086 & & 0.0000 & 0.0070 & & 0.0000 & 0.0110 & & 0.0000 & -0.0177 & & 0.0000 & 0.0000 & 0.0000 \\
\hline & $5 / 13 / 2015$ & 5 & 0.0085 & \begin{tabular}{|c|}
-0.0001 \\
\end{tabular} & -0.0010 & 0.0079 & 0.0009 & 0.0090 & 0.0117 & 0.0007 & 0.0070 & -0.0177 & 0.0000 & 0.0000 & 0.0038 & 0.0050 \\
\hline & $5 / 22 / 2015$ & 14 & 0.0098 & 0.0012 & 0.0120 & 0.0092 & 0.0022 & 0.0220 & 0.0130 & 0.0020 & 0.0200 & -0.0162 & 0.0015 & 0.0150 & 0.0173 & 0.0046 \\
\hline & $5 / 29 / 2015$ & 21 & 0.0115 & 0.0029 & 0.0290 & 0.0094 & 0.0024 & 0.0240 & 0.0140 & 0.0030 & 0.0300 & -0.0149 & 0.0028 & 0.0280 & 0.0278 & 0.0026 \\
\hline & $6 / 5 / 2015$ & 28 & 0.0122 & 0.0036 & 0.0360 & 0.0109 & 0.0039 & 0.0390 & 0.0160 & 0.0050 & 0.0500 & -0.0134 & 0.0043 & 0.0430 & 0.0420 & 0.0061 \\
\hline & $6 / 7 / 2015$ & 30 & 0.0125 & 0.0039 & 0.0390 & 0.0111 & 0.0041 & 0.0410 & 0.0162 & 0.0052 & 0.0520 & \begin{tabular}{|c|}
-0.0132 \\
\end{tabular} & 0.0045 & 0.0450 & 0.0443 & 0.0057 \\
\hline
\end{tabular}

ASTM C 1567 Armstrong Cement with Hanson Aggregate and Sammis Fly Ash- "Mix 5"

Charleroi Lock and Dam Project

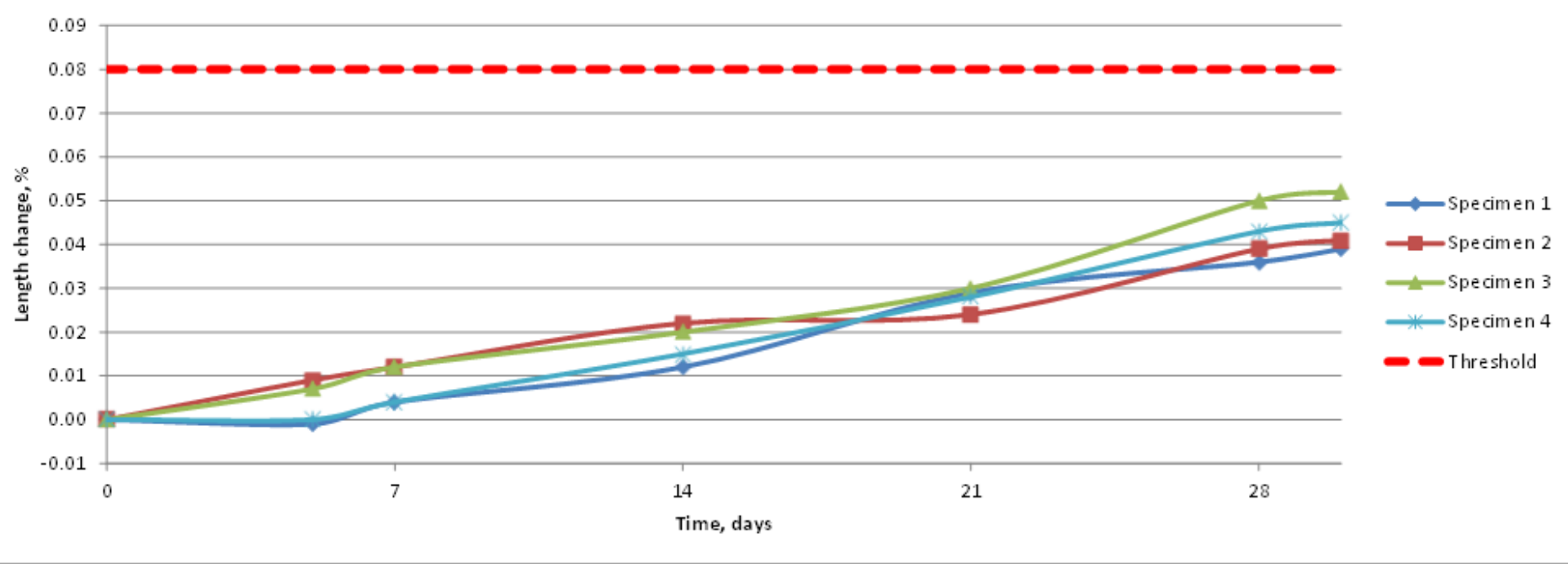




\begin{tabular}{|c|c|c|c|c|c|c|c|c|c|c|c|c|c|c|c|c|}
\hline Mix: & \multicolumn{5}{|c|}{ Mix 5 with Hanson Aggregate and Ft. Martin Fly Ash } & & & & & & & & & & & \\
\hline \multicolumn{17}{|c|}{\begin{tabular}{|l|l} 
Potential Alkali Reactivity of Aggregates, ASTM C 1567 \\
\end{tabular}} \\
\hline & Date & & \multicolumn{3}{|c|}{ Specimen 1} & \multicolumn{3}{|c|}{ Specimen 2} & \multicolumn{3}{|c|}{ Specimen 3} & \multicolumn{3}{|c|}{ Specimen 4} & Average & Standard \\
\hline Mixture & & Age $^{*}$ & Reading & Change & Expansion & Reading & Change & Expansion & Reading & Change & Expans ion & Reading & Change & Expans ior & Expansion & Deviation \\
\hline ID & $\mathrm{D} / \mathrm{M} / \mathrm{Yr}$ & (day) & (in.) & (in.) & $(\%)$ & (in.) & (in.) & $(\%)$ & (in.) & (in.) & $(\%)$ & (in.) & (in.) & $(\%)$ & $(\%)$ & $(\%)$ \\
\hline Mix & $4 / 28 / 2015$ & & - & - & - & - & - & - & - & - & - & - & - & - & - & - \\
\hline Initial & $4 / 30 / 2015$ & & -0.0068 & & & -0.0131 & & & 0.0313 & & & -0.0166 & & & & \\
\hline \multirow[t]{7}{*}{ zero read } & $5 / 1 / 2015$ & 0 & -0.0008 & & 0.0000 & -0.0074 & & 0.0000 & 0.0371 & & 0.0000 & -0.0108 & & 0.0000 & 0.0000 & 0.0000 \\
\hline & $5 / 6 / 2015$ & 5 & -0.0003 & 0.0005 & 0.0050 & \begin{tabular}{|c|}
-0.0071 \\
\end{tabular} & 0.0003 & 0.0030 & 0.0373 & 0.0002 & 0.0020 & -0.0106 & 0.0002 & 0.0020 & 0.0030 & 0.0014 \\
\hline & $5 / 8 / 2015$ & 7 & 0.0003 & 0.0011 & 0.0110 & -0.0065 & 0.0009 & 0.0090 & 0.0376 & 0.0005 & 0.0050 & -0.0104 & 0.0004 & 0.0040 & 0.0073 & 0.0033 \\
\hline & $5 / 15 / 2015$ & 14 & 0.0013 & 0.0021 & 0.0210 & -0.0062 & 0.0012 & 0.0120 & 0.0387 & 0.0016 & 0.0160 & -0.0094 & 0.0014 & 0.0140 & 0.0158 & 0.0039 \\
\hline & $5 / 22 / 2015$ & 21 & 0.0023 & 0.0031 & 0.0310 & -0.0048 & 0.0026 & 0.0260 & 0.0397 & 0.0026 & 0.0260 & -0.0084 & 0.0024 & 0.0240 & 0.0268 & 0.0030 \\
\hline & $5 / 29 / 2015$ & 28 & 0.0040 & 0.0048 & 0.0480 & -0.0025 & 0.0049 & 0.0490 & 0.0415 & 0.0044 & 0.0440 & -0.0063 & 0.0045 & 0.0450 & 0.0465 & 0.0024 \\
\hline & $5 / 31 / 2015$ & 30 & 0.0042 & 0.0050 & 0.0500 & \begin{tabular}{|c|}
-0.0027 \\
\end{tabular} & 0.0047 & 0.0470 & 0.0417 & 0.0046 & 0.0460 & -0.0063 & 0.0045 & 0.0450 & 0.0470 & 0.0022 \\
\hline
\end{tabular}

ASTM C 1567 Armstrong Cement with Hanson Aggregate and Ft. Martin Fly Ash- "Mix 5"

Charleroi Lock and Dam Project

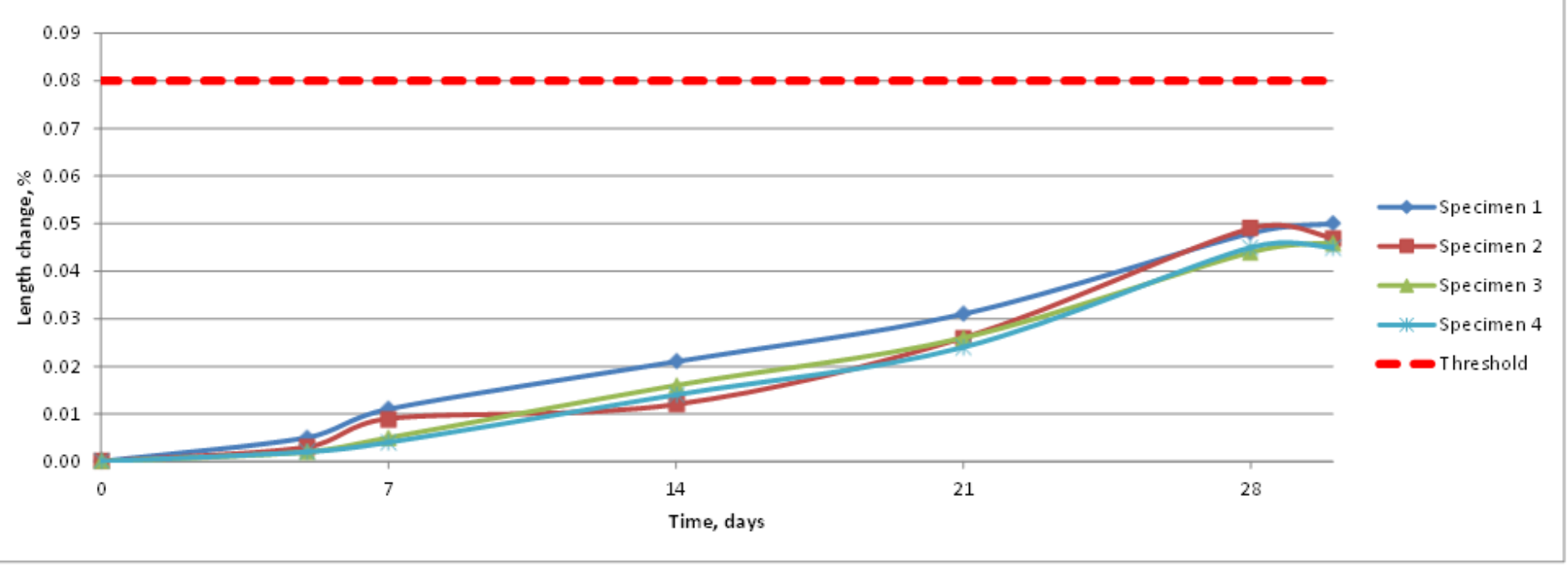




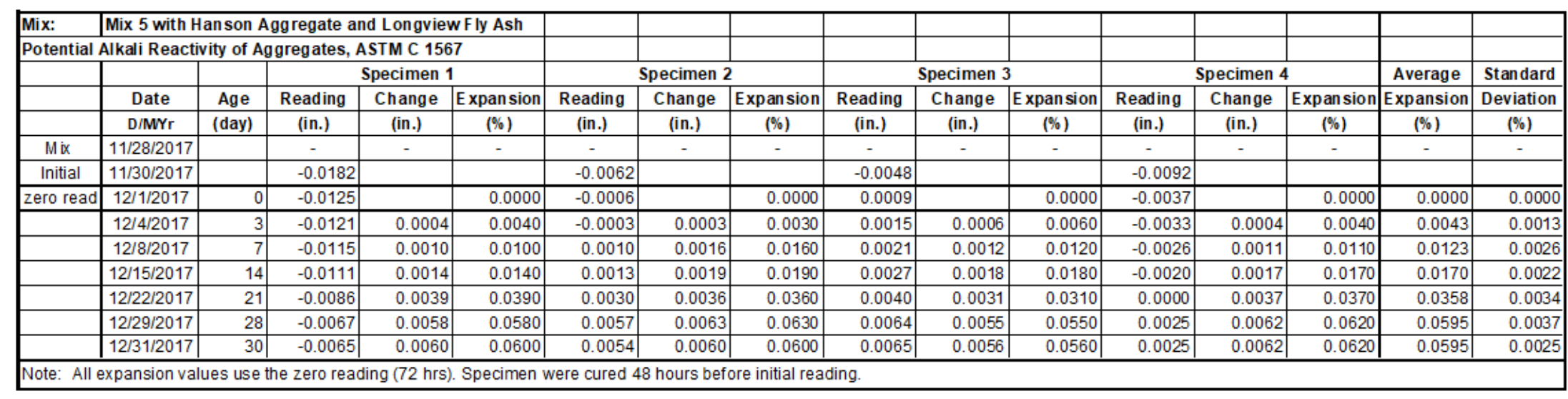

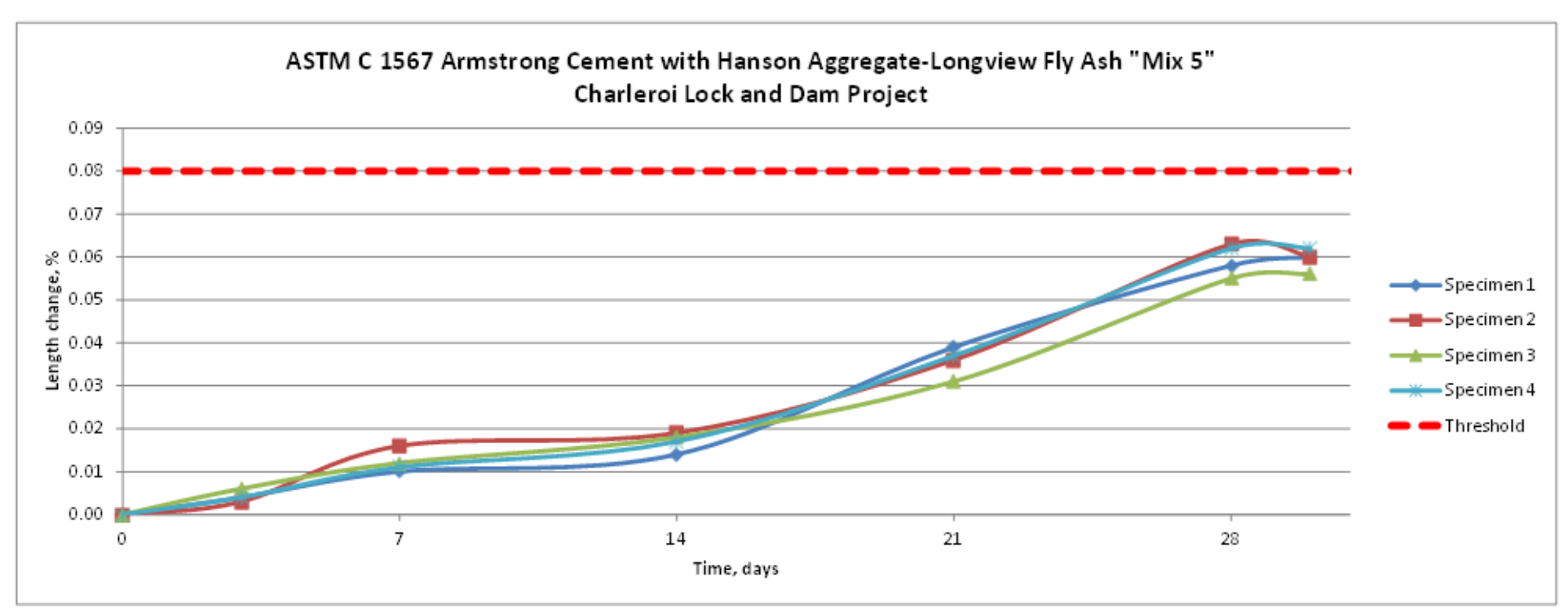




\begin{tabular}{|c|c|c|c|c|c|c|c|c|c|c|c|c|c|}
\hline Mix: & \multicolumn{13}{|c|}{ Mix 5 with Hanson Aggregate and Brandon Shores Fly Ash } \\
\hline \multicolumn{14}{|c|}{ Potential Alkali Reactivity of Aggregates, ASTM C 1567} \\
\hline & & & \multicolumn{3}{|c|}{ Specimen 1} & \multicolumn{3}{|c|}{ Specimen 2} & \multicolumn{3}{|c|}{ Specimen 3} & Average & Standard \\
\hline & Date & Age & Reading & Change & Expansion & Reading & Change & Expansion & Reading & Change & Expansion & Expansion & Deviation \\
\hline & $\mathrm{D} / \mathrm{MYr}$ & (day) & (in.) & (in.) & $(\%)$ & (in.) & (in.) & $(\%)$ & (in.) & (in.) & $(\%)$ & $(\%)$ & $(\%)$ \\
\hline Mix & $3 / 20 / 2018$ & & - & - & - & - & - & - & - & - & - & - & - \\
\hline Initial & $3 / 22 / 2018$ & & -0.0066 & & & -0.0038 & & & -0.0043 & & & & \\
\hline \multirow[t]{7}{*}{ zero read } & $3 / 23 / 2018$ & 0 & 0.0003 & & 0.0000 & 0.0025 & & 0.0000 & 0.0010 & & 0.0000 & 0.0000 & 0.0000 \\
\hline & $3 / 26 / 2018$ & 3 & 0.0005 & 0.0002 & \begin{tabular}{l|l|}
0.0020 \\
\end{tabular} & 0.0028 & 0.0003 & 0.0030 & 0.0013 & 0.0003 & $\begin{array}{ll}0.0030 \\
\end{array}$ & 0.0027 & 0.0006 \\
\hline & $3 / 28 / 2018$ & 5 & 0.0010 & 0.0007 & \begin{tabular}{l|l}
0.0070 \\
\end{tabular} & 0.0030 & 0.0005 & 0.0050 & 0.0015 & 0.0005 & 0.0050 & 0.0057 & 0.0012 \\
\hline & $3 / 30 / 2018$ & 7 & 0.0014 & 0.0011 & 0.0110 & 0.0033 & \begin{tabular}{l|l}
0.0008 \\
\end{tabular} & 0.0080 & 0.0026 & 0.0016 & $\begin{array}{ll}0.0160 \\
\end{array}$ & 0.0117 & 0.0040 \\
\hline & $4 / 6 / 2018$ & 14 & 0.0038 & 0.0035 & 0.0350 & 0.0055 & 0.0030 & 0.0300 & 0.0044 & 0.0034 & 0.0340 & 0.0330 & 0.0026 \\
\hline & $4 / 20 / 2018$ & 28 & 0.0048 & 0.0045 & \begin{tabular}{l|l}
0.0450 \\
\end{tabular} & 0.0065 & \begin{tabular}{l|l}
0.0040 \\
\end{tabular} & 0.0400 & 0.0053 & 0.0043 & \begin{tabular}{l|l}
0.0430 \\
\end{tabular} & 0.0427 & 0.0025 \\
\hline & $4 / 23 / 2018$ & 30 & 0.0050 & 0.0047 & \begin{tabular}{|c|}
0.0470 \\
\end{tabular} & 0.0066 & 0.0041 & 0.0410 & 0.0054 & 0.0044 & \begin{tabular}{|l|}
0.0440 \\
\end{tabular} & 0.0440 & 0.0030 \\
\hline
\end{tabular}

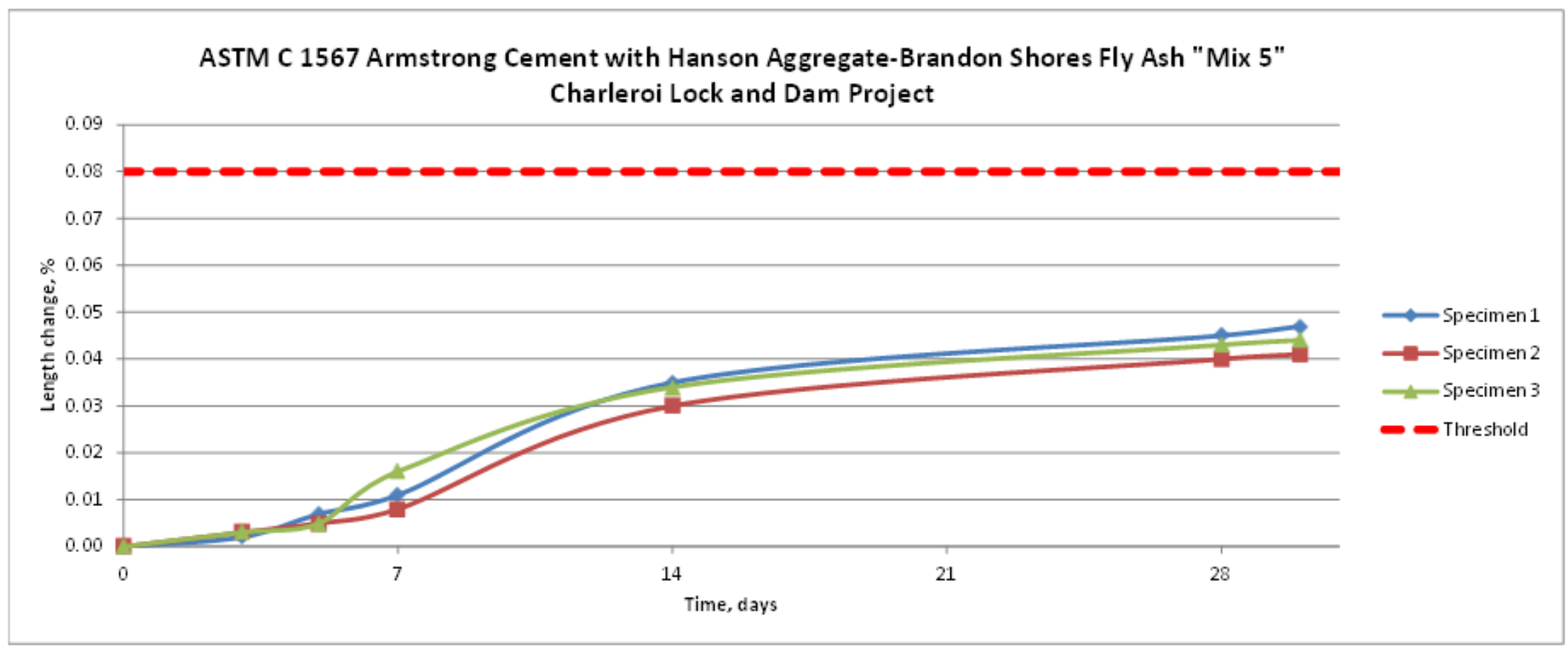




\begin{tabular}{|c|c|c|c|c|c|c|c|c|c|c|c|c|c|c|c|c|}
\hline Mix: & \multicolumn{6}{|c|}{ Mix 5 with Georgetown Aggregate and Sammis Fly Ash } & & & & & & & & & & \\
\hline \multicolumn{17}{|c|}{ Potential Alkali Reactivity of Aggregates, ASTM C 1567} \\
\hline & Date & & \multicolumn{3}{|c|}{ Specimen 1} & \multicolumn{3}{|c|}{ Specimen 2} & \multicolumn{3}{|c|}{ Specimen 3} & \multicolumn{3}{|c|}{ Specimen 4} & Average & Standard \\
\hline Mixture & & Age $^{*}$ & Reading & Change & Expansion & Reading & Change & Expansion & Reading & Change & Expans ion & Reading & Change & Expans ior & Expans ion & Deviation \\
\hline ID & $\mathrm{D} / \mathrm{M} / \mathrm{Yr}$ & (day) & (in.) & (in.) & $(\%)$ & (in.) & (in.) & $(\%)$ & (in.) & (in.) & $(\%)$ & (in.) & (in.) & $(\%)$ & $(\%)$ & $(\%)$ \\
\hline Mix & $4 / 21 / 2015$ & & - & - & - & - & - & - & - & - & - & - & - & - & - & - \\
\hline Initial & $4 / 23 / 2015$ & & 0.0002 & & & -0.0012 & & & 0.0009 & & & 0.0049 & & & & \\
\hline zero read & $4 / 24 / 2015$ & 0 & 0.0070 & & 0.0000 & 0.0054 & & 0.0000 & 0.0078 & & 0.0000 & 0.0118 & & 0.0000 & 0.0000 & 0.0000 \\
\hline & $4 / 27 / 2015$ & 3 & 0.0070 & 0.0000 & 0.0000 & 0.0053 & -0.0001 & -0.0010 & 0.0079 & 0.0001 & 0.0010 & 0.0118 & 0.0000 & 0.0000 & 0.0000 & 0.0008 \\
\hline & $5 / 1 / 2015$ & 7 & 0.0077 & 0.0007 & 0.0070 & 0.0058 & 0.0004 & 0.0040 & 0.0084 & 0.0006 & 0.0060 & 0.0122 & 0.0004 & 0.0040 & 0.0053 & 0.0015 \\
\hline & $5 / 8 / 2015$ & 14 & 0.0079 & 0.0009 & 0.0090 & 0.0062 & 0.0008 & 0.0080 & 0.0087 & 0.0009 & 0.0090 & 0.0126 & 0.0008 & 0.0080 & 0.0085 & 0.0006 \\
\hline & $5 / 15 / 2015$ & 21 & 0.0085 & 0.0015 & 0.0150 & 0.0065 & 0.0011 & 0.0110 & 0.0089 & 0.0011 & 0.0110 & 0.0130 & 0.0012 & 0.0120 & 0.0123 & 0.0019 \\
\hline & $5 / 22 / 2015$ & 28 & 0.0088 & 0.0018 & 0.0180 & 0.0071 & 0.0017 & 0.0170 & 0.0096 & 0.0018 & 0.0180 & 0.0135 & 0.0017 & 0.0170 & 0.0175 & 0.0006 \\
\hline & $5 / 24 / 2015$ & 30 & 0.0089 & 0.0019 & 0.0190 & 0.0072 & 0.0018 & 0.0180 & 0.0097 & 0.0019 & 0.0190 & 0.0136 & 0.0018 & 0.0180 & 0.0185 & 0.0006 \\
\hline
\end{tabular}

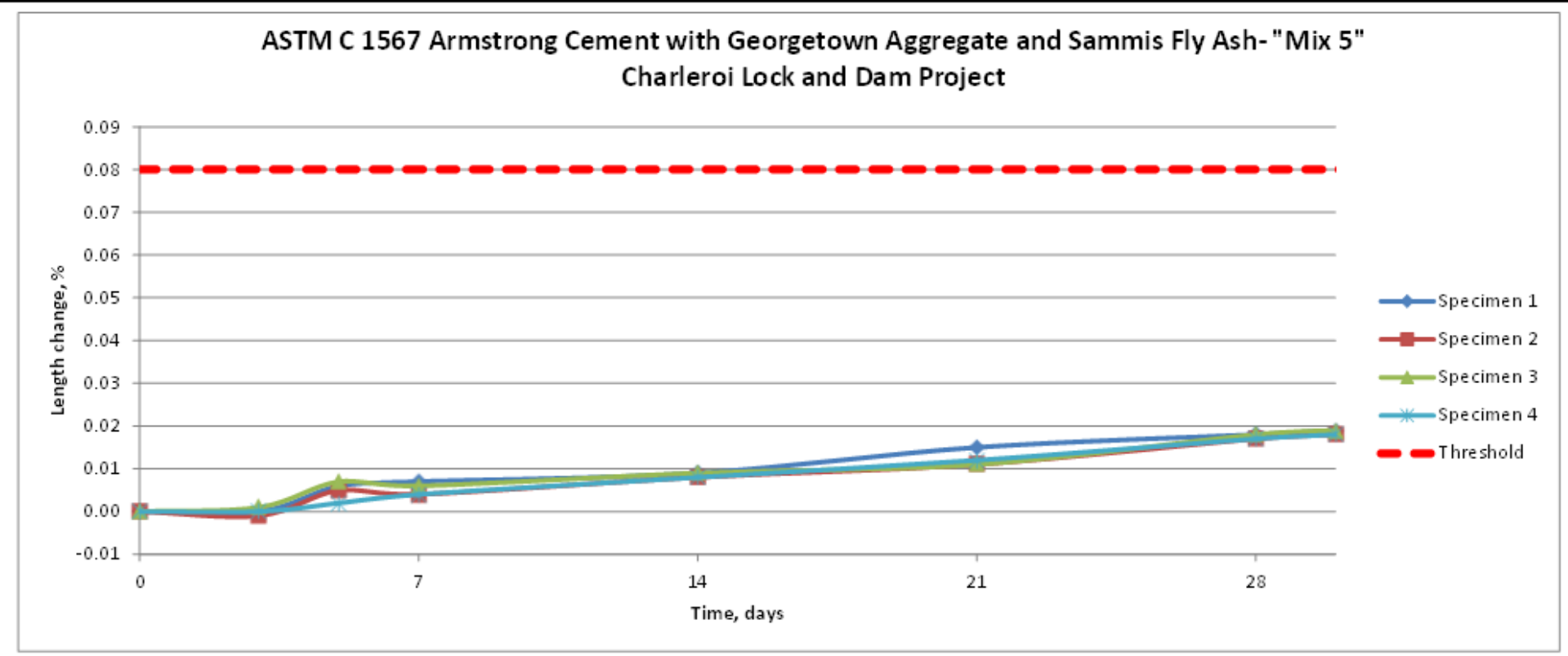




\begin{tabular}{|c|c|c|c|c|c|c|c|c|c|c|c|c|c|}
\hline \begin{tabular}{|l|} 
Mix: \\
Potential
\end{tabular} & \multicolumn{6}{|c|}{ Mix 5 with Georgetown Aggregate and Ft. Martin Fly Ash } & & & & & & & \\
\hline \multicolumn{14}{|c|}{ Potential Alkali Reactivity of Aggregates, ASTM C 1567} \\
\hline & Date & & \multicolumn{3}{|c|}{ Specimen 1} & \multicolumn{3}{|c|}{ Specimen 2} & \multicolumn{3}{|c|}{ Specimen 3} & Average & Standard \\
\hline Mixture & & Age $^{*}$ & Reading & Change & Expansion & Reading & Change & Expansion & Reading & Change & Expansion & Expansior & Deviation \\
\hline ID & $\mathrm{D} / \mathrm{M} / \mathrm{Yr}$ & (day) & (in.) & (in.) & $(\%)$ & (in.) & (in.) & $(\%)$ & (in.) & (in.) & $(\%)$ & $(\%)$ & $(\%)$ \\
\hline Mix & $4 / 28 / 2015$ & & - & - & - & - & - & - & - & - & - & - & - \\
\hline Initial & $4 / 30 / 2015$ & & -0.0026 & & & 0.0025 & & & -0.0022 & & & & \\
\hline \multirow[t]{7}{*}{ zero read } & $5 / 1 / 2015$ & 0 & 0.0042 & & 0.0000 & 0.0043 & & 0.0000 & 0.0042 & & 0.0000 & 0.0000 & 0.0000 \\
\hline & $5 / 6 / 2015$ & 5 & 0.0044 & 0.0002 & 0.0020 & 0.0045 & 0.0002 & 0.0020 & 0.0047 & 0.0005 & 0.0050 & 0.0030 & 0.0017 \\
\hline & $5 / 8 / 2015$ & 7 & 0.0048 & 0.0006 & 0.0060 & 0.0046 & 0.0003 & 0.0030 & 0.0049 & 0.0007 & 0.0070 & 0.0053 & 0.0021 \\
\hline & $5 / 15 / 2015$ & 14 & 0.0052 & 0.0010 & 0.0100 & 0.0047 & 0.0004 & 0.0040 & 0.0048 & 0.0006 & 0.0060 & 0.0067 & 0.0031 \\
\hline & $5 / 22 / 2015$ & 21 & 0.0055 & 0.0013 & 0.0130 & 0.0049 & 0.0006 & 0.0060 & 0.0051 & 0.0009 & 0.0090 & 0.0093 & 0.0035 \\
\hline & $5 / 29 / 2015$ & 28 & 0.0058 & 0.0016 & 0.0160 & 0.0054 & 0.0011 & 0.0110 & 0.0060 & 0.0018 & 0.0180 & 0.0150 & 0.0036 \\
\hline & $5 / 31 / 2015$ & 30 & 0.0060 & 0.0018 & 0.0180 & 0.0057 & 0.0014 & 0.0140 & 0.0061 & 0.0019 & 0.0190 & 0.0170 & 0.0026 \\
\hline
\end{tabular}

- Note: All expansion values use the zero reading (72 hrs). Specimen were cured 48 hours before initial reading. Age (day) represents time of measurement after casting.

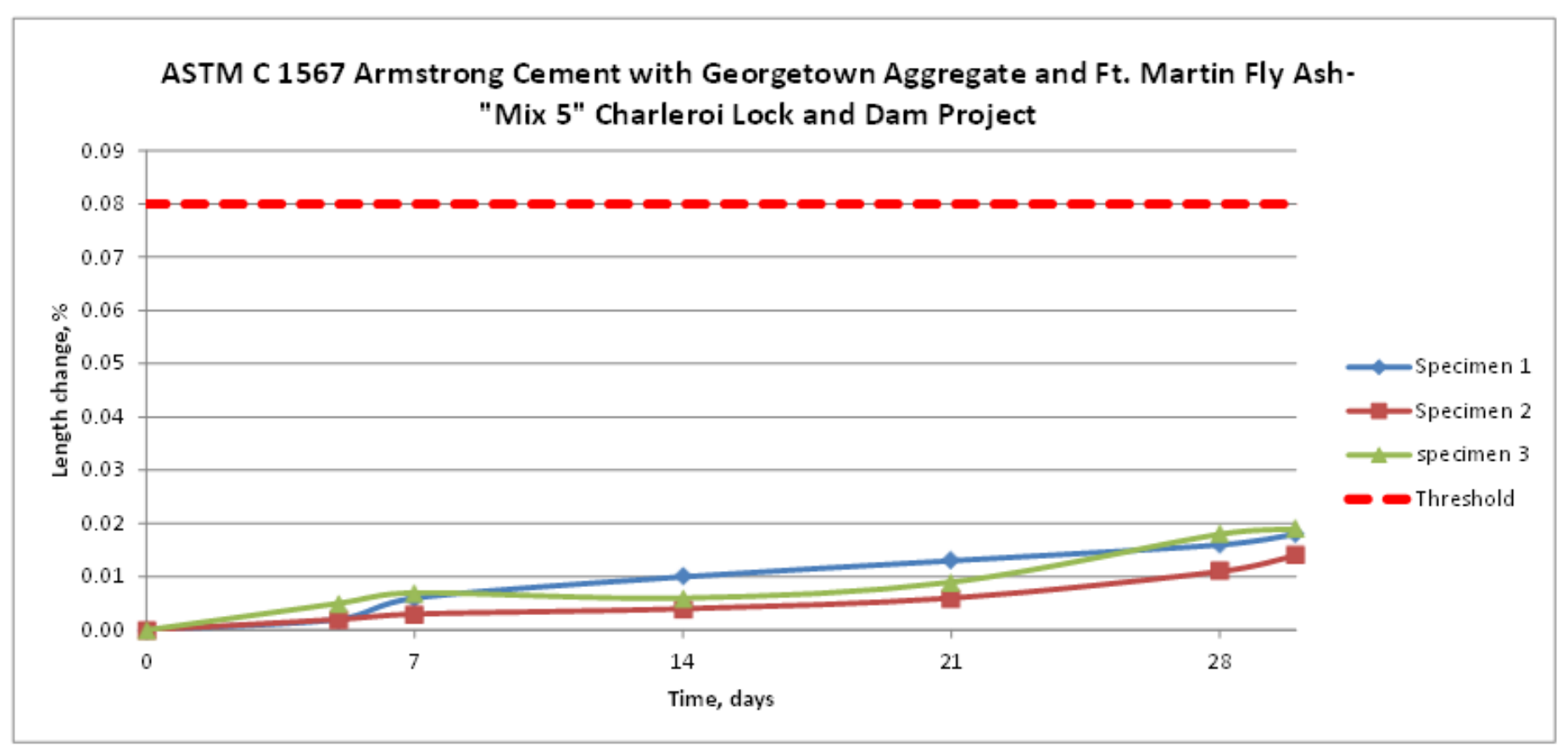




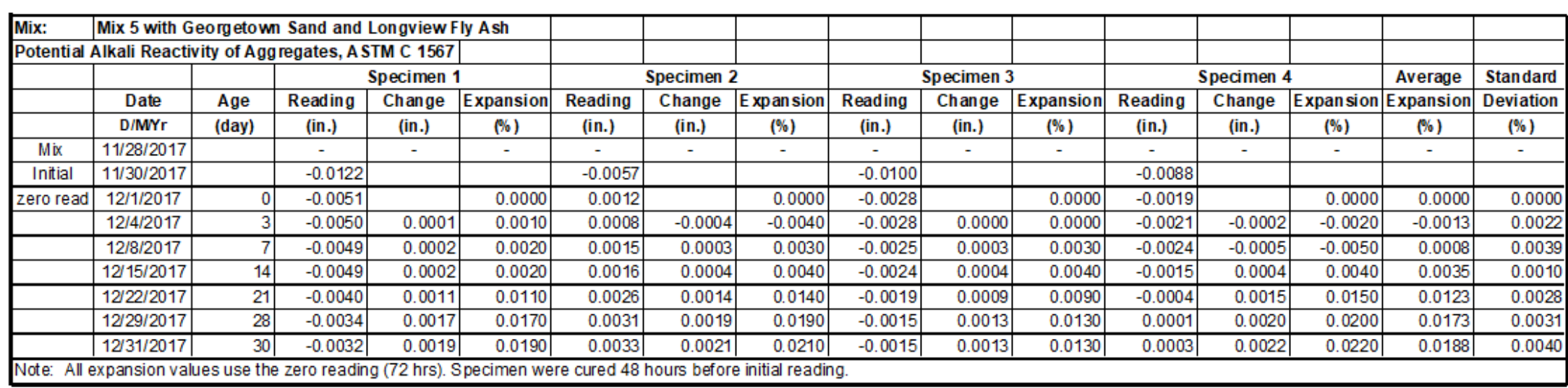

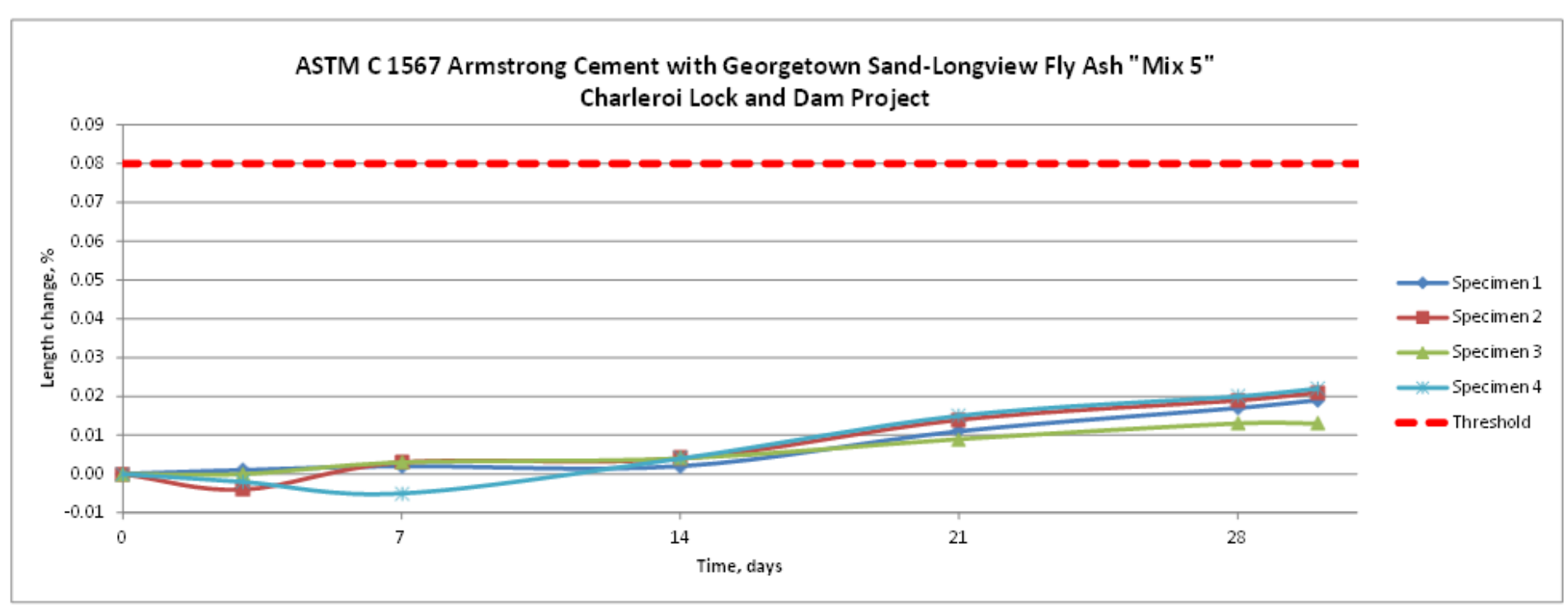




\begin{tabular}{|c|c|c|c|c|c|c|c|c|c|c|c|c|c|c|c|c|}
\hline Mix: & \multicolumn{6}{|c|}{ Mix 5 with Georgetown Sand and Brandon Shores Fly Ash } & & & & & & & & & & \\
\hline \multicolumn{17}{|c|}{ Potential Alkali Reactivity of Aggregates, ASTM C 1567 } \\
\hline & & & \multicolumn{3}{|c|}{ Specimen 1} & \multicolumn{3}{|c|}{ Specimen 2} & \multicolumn{3}{|c|}{ Specimen 3} & \multicolumn{3}{|c|}{ Specimen 4} & Average & Standard \\
\hline & Date & Age & Reading & Change & Expansion & Reading & Change & Expansion & Reading & Change & Expansion & Reading & Change & Expansion & Expansion & Deviation \\
\hline & $\mathrm{D} / \mathrm{M} \mathrm{Mr}$ & (day) & (in.) & (in.) & (\%) & (in.) & (in.) & $(\%)$ & (in.) & (in.) & $(\%)$ & (in.) & (in.) & $(\%)$ & (\%) & $(\%)$ \\
\hline Initial & $3 / 22 / 2018$ & & -0.0151 & & & 0.0019 & & & -0.0103 & & & -0.0134 & & & & \\
\hline zero read & $3 / 23 / 2018$ & 0 & -0.0085 & & 0.0000 & 0.0089 & & 0.0000 & -0.0039 & & 0.0000 & -0.0066 & & 0.0000 & 0.0000 & 0.0000 \\
\hline & $3 / 26 / 2018$ & 3 & -0.0081 & 0.0004 & 0.0040 & 0.0094 & 0.0005 & 0.0050 & -0.0034 & 0.0005 & 0.0050 & -0.0063 & 0.0003 & 0.0030 & 0.0043 & 0.0010 \\
\hline & $3 / 28 / 2018$ & 5 & -0.0078 & 0.0007 & \begin{tabular}{l|l|}
0.0070 \\
\end{tabular} & 0.0095 & 0.0006 & 0.0060 & -0.0031 & 0.0008 & 0.0080 & -0.0059 & 0.0007 & 0.0070 & 0.0070 & 0.0008 \\
\hline & $4 / 6 / 2018$ & 14 & -0.0075 & 0.0010 & 0.0100 & 0.0099 & 0.0010 & 0.0100 & -0.0025 & 0.0014 & 0.0140 & -0.0050 & 0.0016 & 0.0160 & 0.0125 & 0.0030 \\
\hline & $4 / 20 / 2018$ & 28 & -0.0068 & 0.0017 & 0.0170 & 0.0105 & 0.0016 & 0.0160 & -0.0020 & 0.0019 & 0.0190 & -0.0047 & 0.0019 & 0.0190 & 0.0178 & 0.0015 \\
\hline & $4 / 23 / 2018$ & 30 & -0.0066 & 0.0019 & 0.0190 & 0.0108 & 0.0019 & 0.0190 & -0.0019 & 0.0020 & 0.0200 & -0.0045 & 0.0021 & 0.0210 & 0.0198 & 0.0010 \\
\hline
\end{tabular}

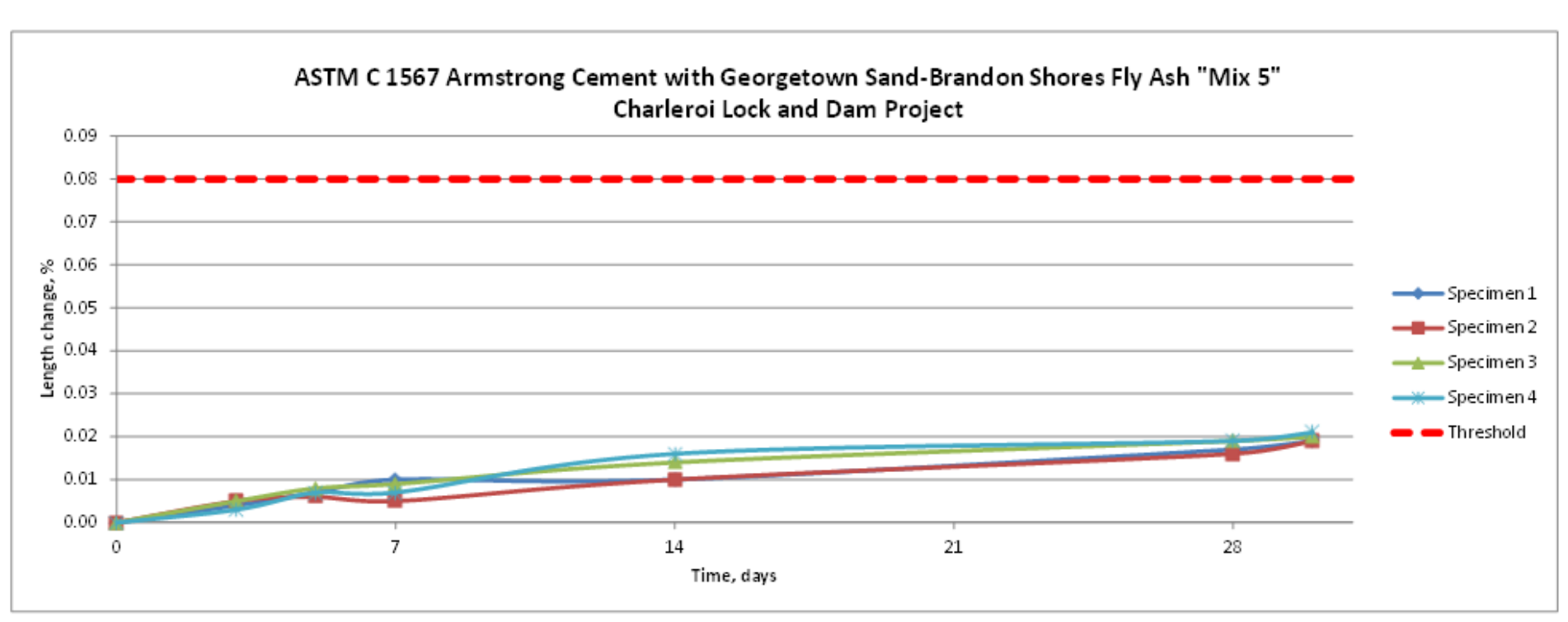




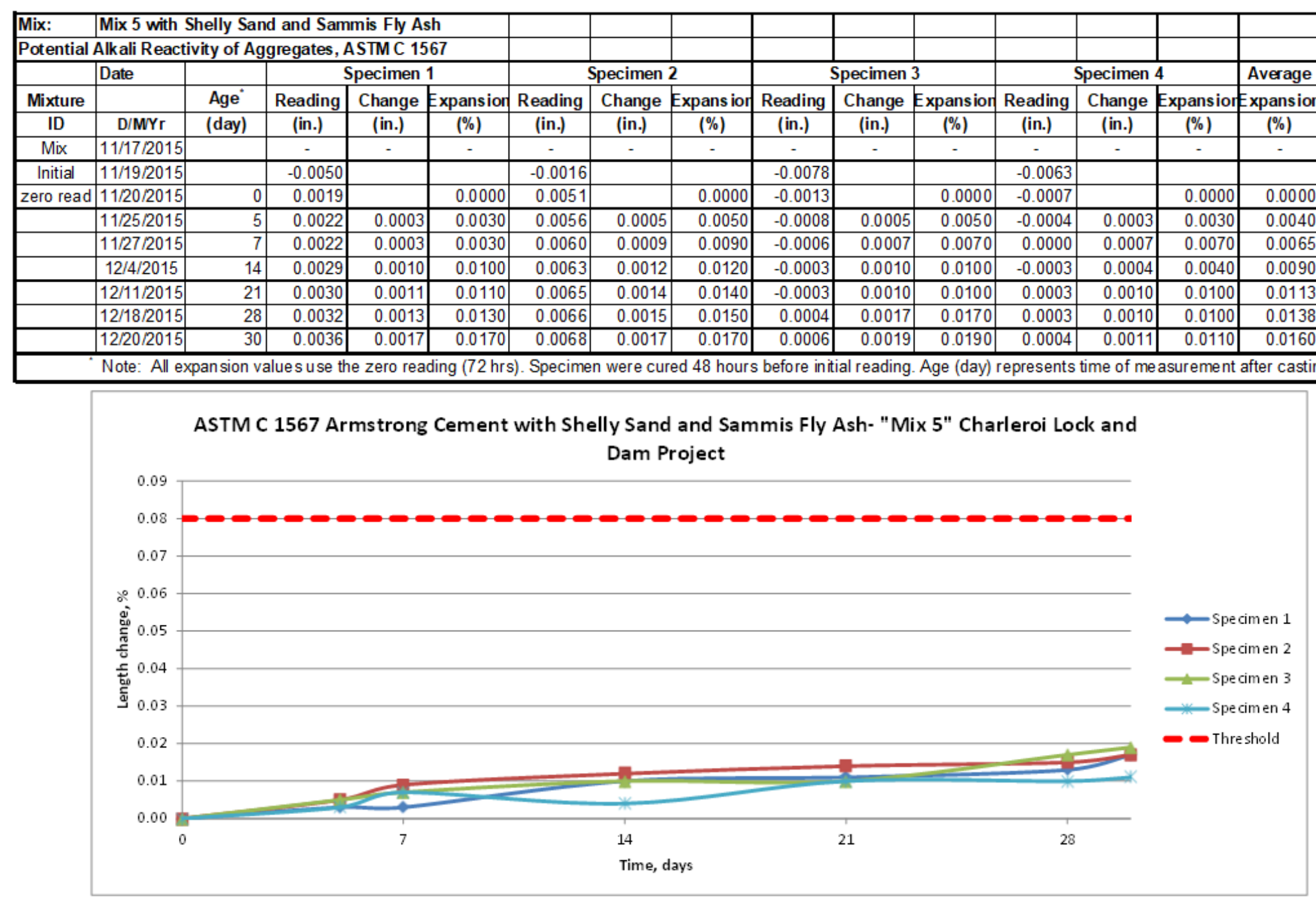




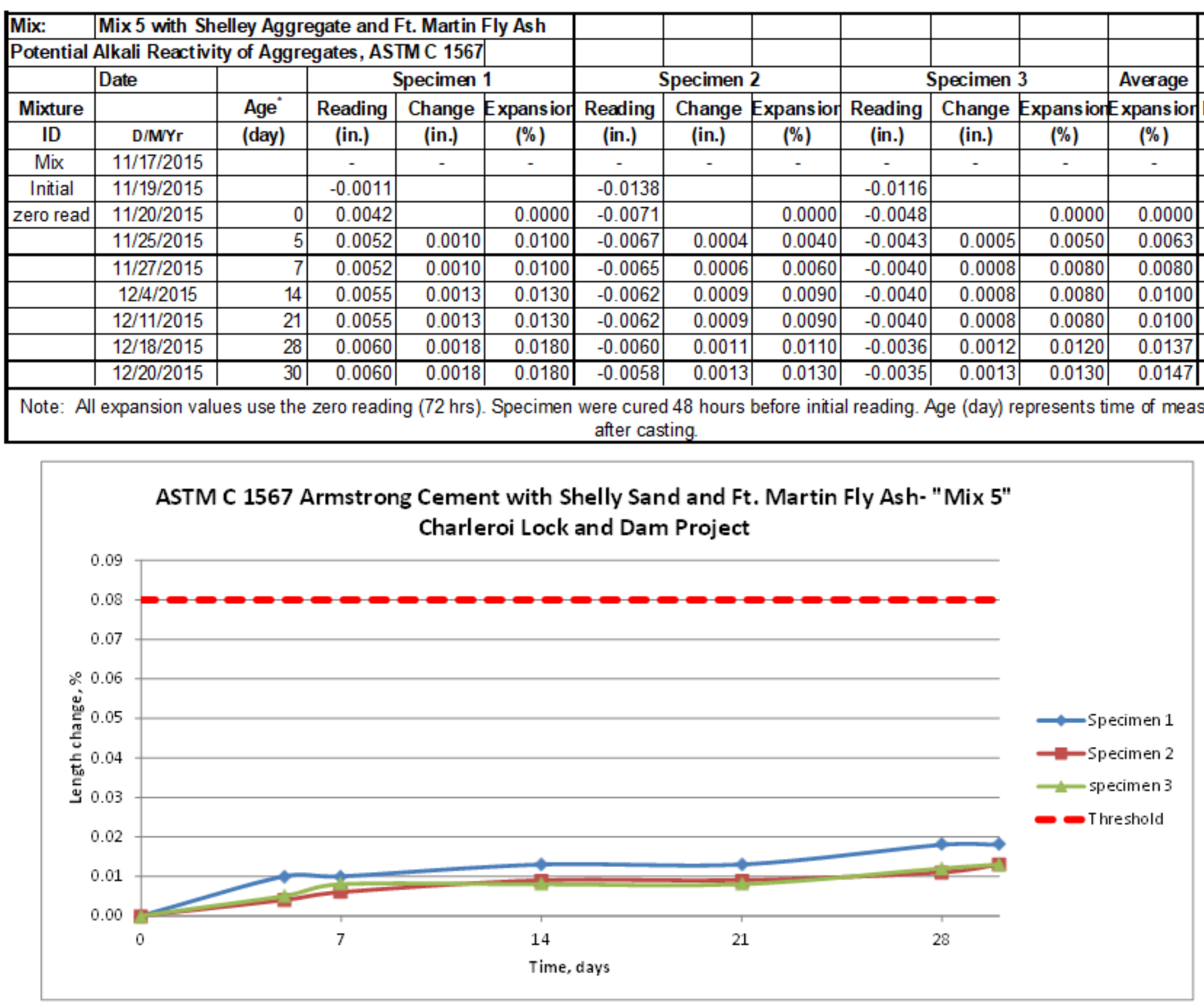




\begin{tabular}{|c|c|c|c|c|c|c|c|c|c|c|c|c|c|c|c|c|}
\hline Mix: & \multicolumn{5}{|c|}{ Mix 5 with Shelley Sand and Brandon Shores Fly Ash } & & & & & & & & & & & \\
\hline \multicolumn{17}{|c|}{\begin{tabular}{|l|l|l|} 
Potential Alkali Reactivity of Agg regates, A STM C 1567 & \\
\end{tabular}} \\
\hline & & & \multicolumn{3}{|c|}{ Specimen 1} & \multicolumn{3}{|c|}{ Specimen 2} & \multicolumn{3}{|c|}{ Specimen 3} & \multicolumn{3}{|c|}{ Specimen 4} & Average & Standard \\
\hline & Date & Age & Reading & Change & Expansion & Reading & Change & Expansion & Reading & Change & Expansion & Reading & Change & Expansion & Expansion & Deviation \\
\hline & DMYr & (day) & (in.) & (in.) & $(\%)$ & (in.) & (in.) & $(\%)$ & (in.) & (in.) & $(\%)$ & (in.) & (in.) & $(\%)$ & $(\%)$ & $(\%)$ \\
\hline$M \dot{M x}$ & $3 / 20 / 2018$ & & - & - & - & - & - & - & - & - & - & - & - & - & - & - \\
\hline Initial & $3 / 22 / 2018$ & & -0.0026 & & & -0.0013 & & & -0.0117 & & & -0.0045 & & & & \\
\hline \multirow[t]{7}{*}{ zero read } & \begin{tabular}{|l|}
$3 / 23 / 2018$ \\
\end{tabular} & 0 & 0.0040 & & 0.0000 & 0.0054 & & 0.0000 & -0.0050 & & 0.0000 & 0.0020 & & 0.0000 & 0.0000 & 0.0000 \\
\hline & $3 / 26 / 2018$ & 3 & 0.0042 & 0.0002 & 0.0020 & 0.0055 & 0.0001 & 0.0010 & -0.0048 & 0.0002 & 0.0020 & 0.0022 & 0.0002 & 0.0020 & 0.0018 & 0.0005 \\
\hline & $3 / 28 / 2018$ & 5 & \begin{tabular}{l|l|}
0.0047 \\
\end{tabular} & 0.0007 & \begin{tabular}{l|l|}
0.0070 \\
\end{tabular} & 0.0063 & 0.0009 & $\begin{array}{l}0.0090 \\
\end{array}$ & -0.0043 & 0.0007 & \begin{tabular}{l|l}
0.0070 \\
\end{tabular} & 0.0029 & $\begin{array}{ll}0.0009 \\
\end{array}$ & 0.0090 & 0.0080 & 0.0012 \\
\hline & $3 / 30 / 2018$ & 7 & 0.0045 & 0.0005 & 0.0050 & 0.0061 & 0.0007 & $\begin{array}{ll}0.0070 \\
\end{array}$ & -0.0043 & 0.0007 & 0.0070 & 0.0029 & 0.0009 & 0.0090 & 0.0070 & 0.0016 \\
\hline & $4 / 6 / 2018$ & 14 & 0.0052 & 0.0012 & 0.0120 & 0.0065 & 0.0011 & \begin{tabular}{l|l}
0.0110 \\
\end{tabular} & -0.0040 & 0.0010 & 0.0100 & 0.0035 & 0.0015 & 0.0150 & 0.0120 & 0.0022 \\
\hline & $4 / 20 / 2018$ & 28 & 0.0057 & 0.0017 & 0.0170 & 0.0070 & 0.0016 & 0.0160 & -0.0035 & 0.0015 & 0.0150 & 0.0040 & 0.0020 & 0.0200 & 0.0170 & 0.0022 \\
\hline & $4 / 23 / 2018$ & 31 & 0.0059 & 0.0019 & 0.0190 & 0.0072 & 0.0018 & \begin{tabular}{l|l|l}
0.0180 \\
\end{tabular} & -0.0031 & 0.0019 & 0.0190 & 0.0041 & 0.0021 & \begin{tabular}{|c|}
0.0210 \\
\end{tabular} & \begin{tabular}{|c|}
0.0193 \\
\end{tabular} & 0.0013 \\
\hline
\end{tabular}

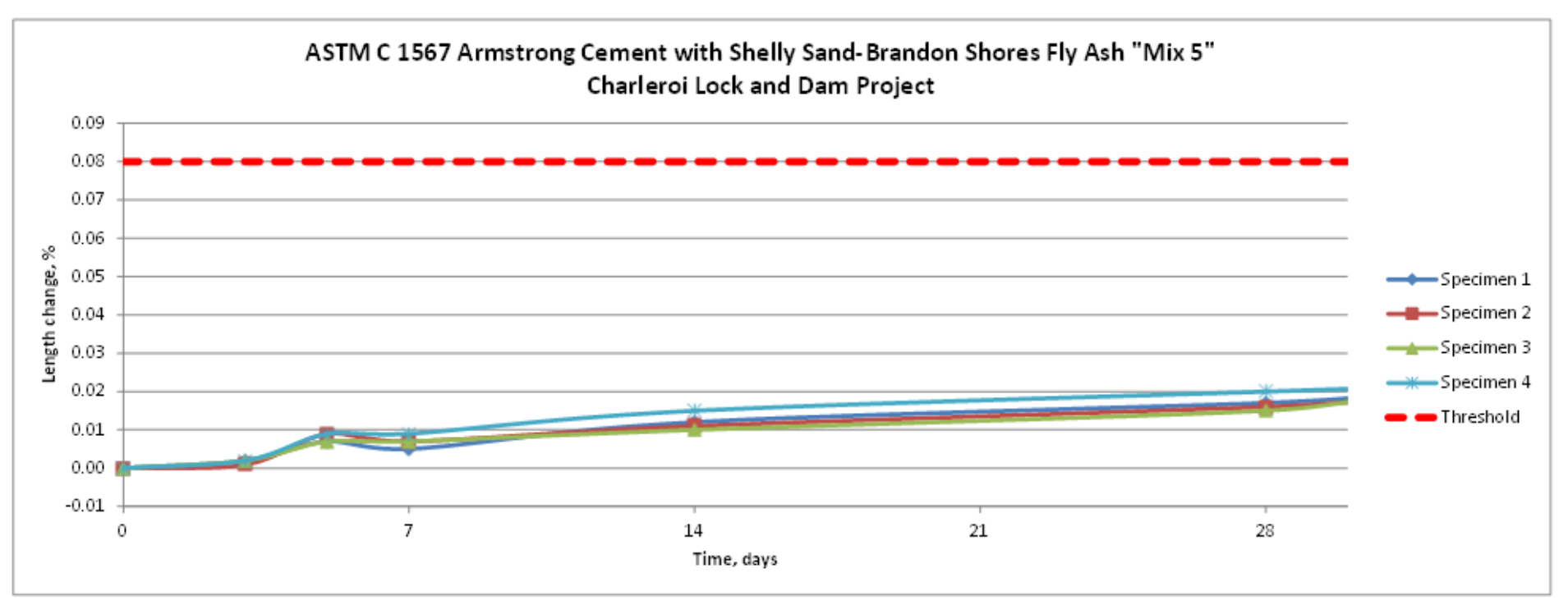




\begin{tabular}{|c|c|c|c|c|c|c|c|c|c|c|c|c|c|c|c|c|}
\hline \multirow{2}{*}{\multicolumn{17}{|c|}{\begin{tabular}{|l|l|} 
Mix: & Mix 5 with Shelley Sand and Longview Fly Ash \\
Potential Alkali Reactivity of Aag reaates. ASTMC 1567 \\
\end{tabular}}} \\
\hline & & & & & \multicolumn{12}{|c|}{\begin{tabular}{|l|l|} 
Potential Alkali Reactivity of Agg regates, ASTM C 1567 & \\
\end{tabular}} \\
\hline & & & \multicolumn{3}{|c|}{ Specimen 1} & \multicolumn{3}{|c|}{ Specimen 2} & \multicolumn{3}{|c|}{ Specimen 3} & \multicolumn{3}{|c|}{ Specimen 4} & Average & Stand ard \\
\hline & Date & Age & Reading & Change & Expansion & Reading & Change & Expansion & Reading & Change & Expansion & Reading & Change & Expansion & Expansion & Deviation \\
\hline & D/MYr & (day) & (in.) & (in.) & $(\%)$ & (in.) & (in.) & $(\%)$ & (in.) & (in.) & $(\%)$ & (in.) & (in.) & $(\%)$ & $(\%)$ & $(\%)$ \\
\hline Initial & $11 / 30 / 2017$ & & -0.0081 & & & -0.0355 & & & -0.0106 & & & -0.0123 & & & & \\
\hline zero read & $12 / 1 / 2017$ & 0 & -0.0012 & & 0.0000 & -0.0286 & & 0.0000 & -0.0038 & & 0.0000 & -0.0058 & & 0.0000 & 0.0000 & 0.0000 \\
\hline & $12 / 4 / 2017$ & 3 & -0.0008 & 0.0004 & 0.0040 & -0.0283 & 0.0003 & 0.0030 & -0.0033 & 0.0005 & 0.0050 & -0.0055 & 0.0003 & 0.0030 & 0.0038 & 0.0010 \\
\hline & $12 / 8 / 2017$ & 7 & -0.0011 & 0.0001 & \begin{tabular}{|l|l|}
0.0010 \\
\end{tabular} & -0.0283 & 0.0003 & 0.0030 & -0.0035 & 0.0003 & 0.0030 & -0.0051 & 0.0007 & 0.0070 & 0.0035 & 0.0025 \\
\hline & $12 / 22 / 2017$ & 21 & 0.0001 & 0.0013 & $\begin{array}{l}0.0130 \\
\end{array}$ & -0.0277 & 0.0009 & 0.0090 & -0.0029 & 0.0009 & 0.0090 & -0.0047 & 0.0011 & 0.0110 & 0.0105 & 0.0019 \\
\hline & $12 / 29 / 2017$ & 28 & 0.0007 & 0.0019 & 0.0190 & -0.0270 & 0.0016 & 0.0160 & -0.0020 & 0.0018 & 0.0180 & -0.0040 & 0.0018 & 0.0180 & 0.0178 & 0.0013 \\
\hline & $12 / 31 / 2017$ & 30 & 0.0008 & 0.0020 & 0.0200 & $\mid-0.0267$ & 0.0019 & 0.0190 & $\mid-0.0018$ & 0.0020 & 0.0200 & -0.0034 & 0.0024 & 0.0240 & 0.0208 & 0.0022 \\
\hline
\end{tabular}

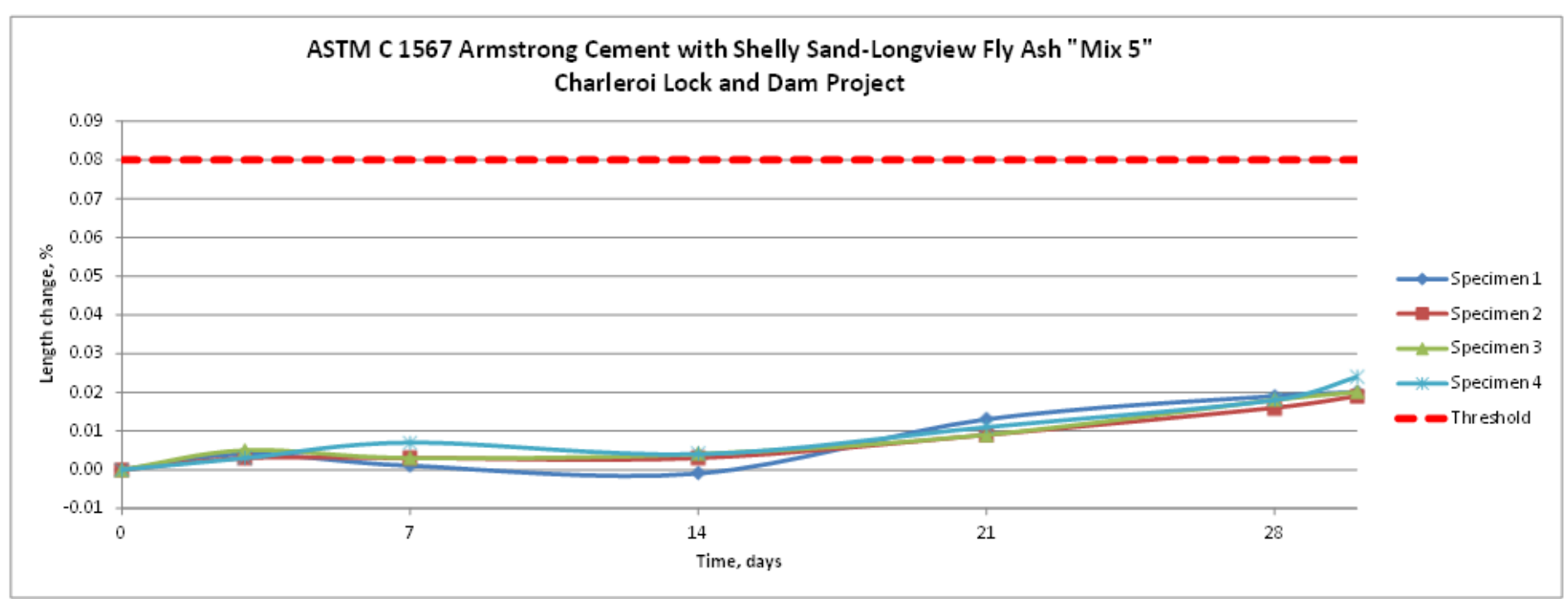




\begin{tabular}{|c|c|c|c|c|c|c|c|c|c|c|c|c|c|c|c|c|}
\hline Mix: & \multicolumn{5}{|c|}{ Mix 7 with Han son Aggregate and Sammis Fly Ash } & & & & & & & & & & & \\
\hline \multicolumn{17}{|c|}{ Potential Alkali Reactivity of Agg regates, ASTM C 1567} \\
\hline & & & \multicolumn{3}{|c|}{ Specimen 1} & \multicolumn{3}{|c|}{ Specimen 2} & \multicolumn{3}{|c|}{ Specimen 3} & \multicolumn{3}{|c|}{ Specimen 4} & Average & Standard \\
\hline & Date & Age & Reading & Change & Expansion & Reading & Change & Expansion & Reading & Change & Expansion & Reading & Change & \begin{tabular}{|l|} 
Expansion \\
\end{tabular} & \begin{tabular}{|l|} 
Expansion \\
\end{tabular} & \begin{tabular}{|l|} 
Deviation \\
\end{tabular} \\
\hline & DMYr & (day) & (in.) & (in.) & $(\%)$ & (in.) & (in.) & $(\%)$ & (in.) & (in.) & $(\%)$ & (in.) & (in.) & $(\%)$ & $(\%)$ & (\%) \\
\hline Initial & $4 / 26 / 2018$ & & -0.0008 & & & -0.0078 & & & -0.0073 & & & -0.0104 & & & & \\
\hline zero read & $4 / 27 / 2018$ & 0 & 0.0065 & & 0.0000 & -0.0009 & & 0.0000 & -0.0006 & & 0.0000 & \begin{tabular}{|c|}
-0.0038 \\
\end{tabular} & & 0.0000 & 0.0000 & 0.0000 \\
\hline & $4 / 30 / 2018$ & 3 & 0.0066 & 0.0001 & 0.0010 & 0.0001 & 0.0010 & 0.0100 & -0.0002 & 0.0004 & 0.0040 & -0.0036 & 0.0002 & 0.0020 & 0.0043 & 0.0040 \\
\hline & $5 / 2 / 2018$ & 5 & 0.0069 & 0.0004 & 0.0040 & 0.0005 & 0.0014 & 0.0140 & 0.0010 & 0.0016 & 0.0160 & -0.0029 & 0.0009 & 0.0090 & \begin{tabular}{|l|l|}
0.0108 \\
\end{tabular} & 0.0054 \\
\hline & $5 / 11 / 2018$ & 14 & 0.0083 & 0.0018 & 0.0180 & 0.0020 & 0.0029 & 0.0290 & 0.0017 & 0.0023 & 0.0230 & -0.0014 & 0.0024 & 0.0240 & 0.0235 & 0.0045 \\
\hline & $5 / 25 / 2018$ & 28 & 0.0094 & 0.0029 & 0.0290 & 0.0026 & 0.0035 & 0.0350 & 0.0027 & 0.0033 & 0.0330 & -0.0010 & 0.0028 & 0.0280 & \begin{tabular}{l|l|l}
0.0313 \\
\end{tabular} & 0.0033 \\
\hline & $5 / 28 / 2018$ & 31 & 0.0096 & 0.0031 & 0.0310 & 0.0027 & 0.0036 & 0.0360 & 0.0029 & 0.0035 & 0.0350 & -0.0007 & 0.0031 & 0.0310 & 0.0333 & 0.0026 \\
\hline
\end{tabular}

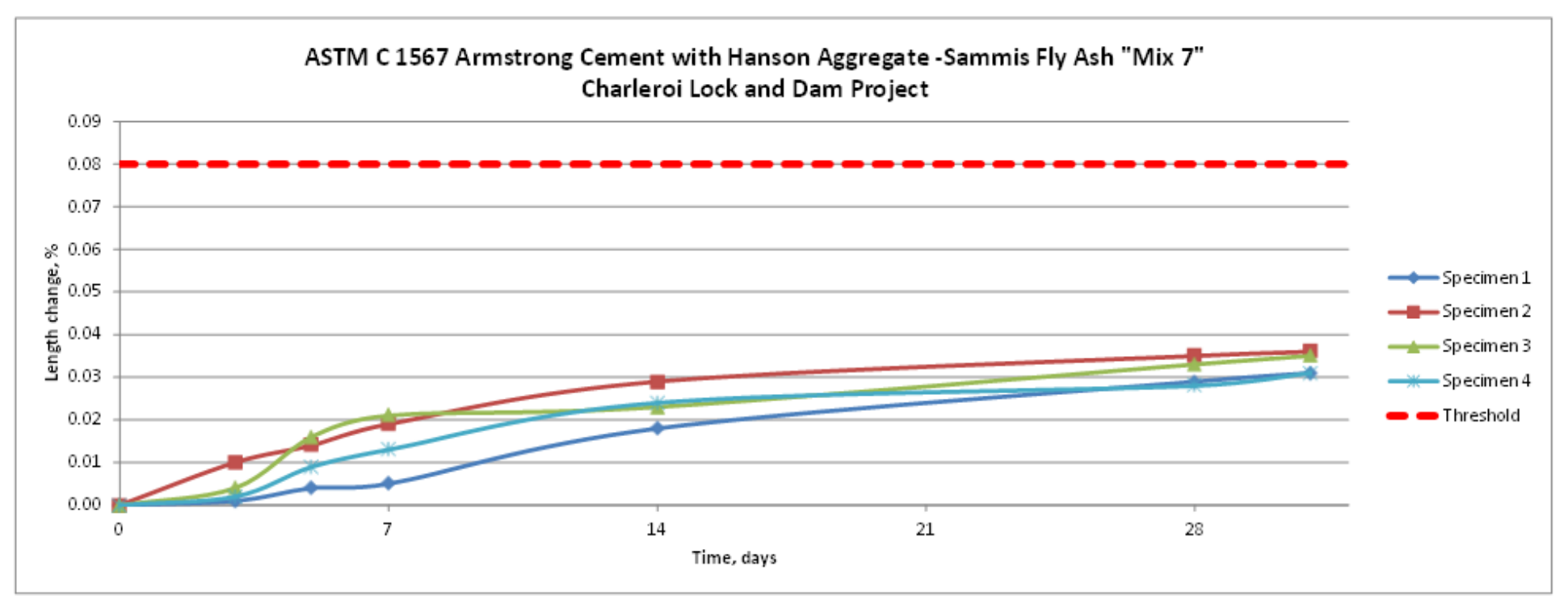




\begin{tabular}{|c|c|c|c|c|c|c|c|c|c|c|c|c|c|c|c|c|}
\hline Mix: & \multicolumn{5}{|c|}{ Mix 7 with Hanson Aggregate and F t. Martin Fly Ash } & & & & & & & & & & & \\
\hline \multicolumn{17}{|c|}{\begin{tabular}{|l|} 
Potential Alkali Reactivity of Agg regates, ASTM C 1567 \\
\end{tabular}} \\
\hline & & & \multicolumn{3}{|c|}{ Specimen 1} & \multicolumn{3}{|c|}{ Specimen 2} & \multicolumn{3}{|c|}{ Specimen 3} & \multicolumn{3}{|c|}{ Specimen 4} & Average & Standard \\
\hline & Date & Age & Reading & Change & Expansion & Reading & Change & Expansion & Reading & Change & Expansion & Reading & Change & Expansion & Expansion & Deviation \\
\hline & D/MYr & (day) & (in.) & (in.) & $(\%)$ & (in.) & (in.) & $(\%)$ & (in.) & (in.) & $(\%)$ & (in.) & (in.) & $(\%)$ & $(\%)$ & $(\%)$ \\
\hline Mix & $5 / 1 / 2018$ & & - & - & - & - & - & - & - & - & - & - & - & - & - & - \\
\hline Initial & $5 / 3 / 2018$ & & -0.0080 & & & -0.0015 & & & 0.0089 & & & 0.0008 & & & & \\
\hline zero read & $5 / 4 / 2018$ & 0 & -0.0015 & & 0.0000 & 0.0055 & & 0.0000 & \begin{tabular}{ll|}
0.0163 \\
\end{tabular} & & 0.0000 & 0.0077 & & 0.0000 & 0.0000 & 0.0000 \\
\hline & 5/7/2018 & 3 & -0.0013 & 0.0002 & 0.0020 & 0.0056 & 0.0001 & 0.0010 & 0.0165 & 0.0002 & 0.0020 & 0.0078 & 0.0001 & 0.0010 & 0.0015 & 0.0006 \\
\hline & $5 / 9 / 2018$ & 5 & -0.0002 & 0.0013 & 0.0130 & 0.0063 & 0.0008 & 0.0080 & 0.0169 & 0.0006 & 0.0060 & 0.0083 & 0.0006 & 0.0060 & 0.0083 & 0.0033 \\
\hline & $5 / 11 / 2018$ & 7 & 0.0005 & 0.0020 & 0.0200 & 0.0070 & 0.0015 & 0.0150 & 0.0175 & 0.0012 & 0.0120 & 0.0090 & 0.0013 & 0.0130 & 0.0150 & 0.0036 \\
\hline & $5 / 18 / 2018$ & 14 & 0.0012 & 0.0027 & 0.0270 & 0.0075 & 0.0020 & 0.0200 & 0.0180 & 0.0017 & 0.0170 & 0.0098 & 0.0021 & 0.0210 & 0.0213 & 0.0042 \\
\hline & $6 / 1 / 2018$ & 28 & 0.0022 & 0.0037 & 0.0370 & 0.0084 & 0.0029 & 0.0290 & 0.0185 & 0.0022 & 0.0220 & 0.0108 & 0.0031 & 0.0310 & \begin{tabular}{l|l}
0.0298 \\
\end{tabular} & 0.0062 \\
\hline & $6 / 4 / 2018$ & 30 & 0.0023 & 0.0038 & 0.0380 & 0.0088 & 0.0033 & 0.0330 & 0.0190 & 0.0027 & 0.0270 & 0.0112 & 0.0035 & 0.0350 & 0.0333 & 0.0046 \\
\hline
\end{tabular}

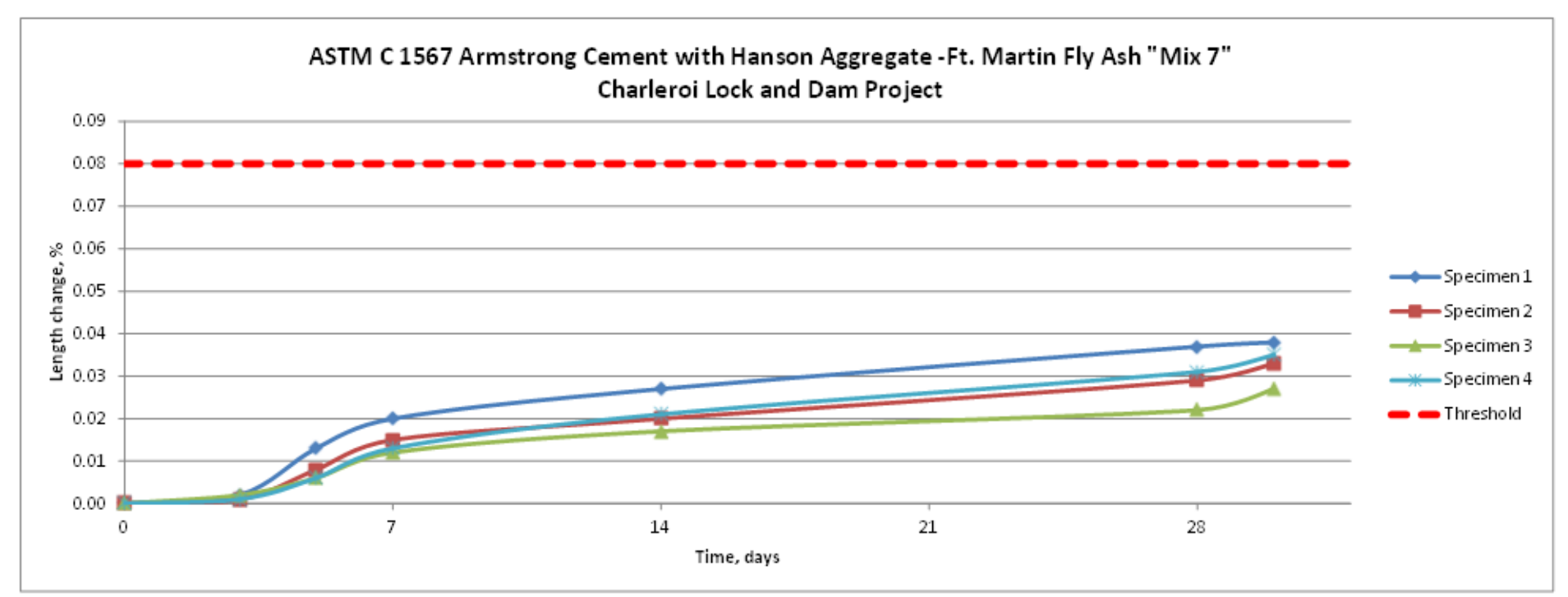




\begin{tabular}{|c|c|c|c|c|c|c|c|c|c|c|c|c|c|}
\hline Mix: & \multicolumn{13}{|c|}{ Mix 7 with Hanson Aggregate and Longview Fly Ash } \\
\hline \multicolumn{14}{|c|}{ Potential Alkali Reactivity of Aggregates, ASTM C 1567} \\
\hline & & & \multicolumn{3}{|c|}{ Specimen 1} & \multicolumn{3}{|c|}{ Specimen 2} & \multicolumn{3}{|c|}{ Specimen 3} & Average & Standard \\
\hline & Date & Age & Reading & Change & Expansion & Reading & Change & Expansion & Reading & Change & Expansion & Expansion & Deviation \\
\hline & $\mathrm{D} / \mathrm{M} / \mathrm{Yr}$ & (day) & (in.) & (in.) & $(\%)$ & (in.) & (in.) & $(\%)$ & (in.) & (in.) & $(\%)$ & $(\%)$ & $(\%)$ \\
\hline Mix & $11 / 28 / 2017$ & & - & - & - & - & - & - & - & - & - & - & - \\
\hline Initial & $11 / 30 / 2017$ & & -0.0075 & & & -0.0412 & & & \begin{tabular}{l|}
-0.0102 \\
\end{tabular} & & & & \\
\hline zero read & $12 / 1 / 2017$ & 0 & -0.0013 & & 0.0000 & -0.0352 & & 0.0000 & -0.0040 & & 0.0000 & 0.0000 & 0.0000 \\
\hline & $12 / 4 / 2017$ & 3 & -0.0011 & 0.0002 & 0.0020 & -0.0352 & 0.0000 & 0.0000 & -0.0038 & 0.0002 & 0.0020 & 0.0013 & 0.0012 \\
\hline & $12 / 8 / 2017$ & 7 & -0.0008 & 0.0005 & 0.0050 & -0.0351 & 0.0001 & 0.0010 & -0.0035 & 0.0005 & 0.0050 & 0.0037 & 0.0023 \\
\hline & $12 / 15 / 2017$ & 14 & 0.0001 & 0.0014 & 0.0140 & -0.0340 & 0.0012 & 0.0120 & -0.0025 & 0.0015 & 0.0150 & 0.0137 & 0.0015 \\
\hline & $12 / 22 / 2017$ & 21 & 0.0019 & 0.0032 & 0.0320 & -0.0324 & 0.0028 & 0.0280 & -0.0015 & 0.0025 & 0.0250 & 0.0283 & 0.0035 \\
\hline & $12 / 29 / 2017$ & 28 & 0.0024 & 0.0037 & 0.0370 & -0.0316 & 0.0036 & \begin{tabular}{l|l}
0.0360 \\
\end{tabular} & 0.0001 & 0.0041 & \begin{tabular}{l|l}
0.0410 \\
\end{tabular} & 0.0380 & 0.0026 \\
\hline & $12 / 31 / 2017$ & 30 & 0.0024 & 0.0037 & 0.0370 & -0.0320 & 0.0032 & 0.0320 & -0.0004 & 0.0036 & 0.0360 & 0.0350 & 0.0026 \\
\hline
\end{tabular}

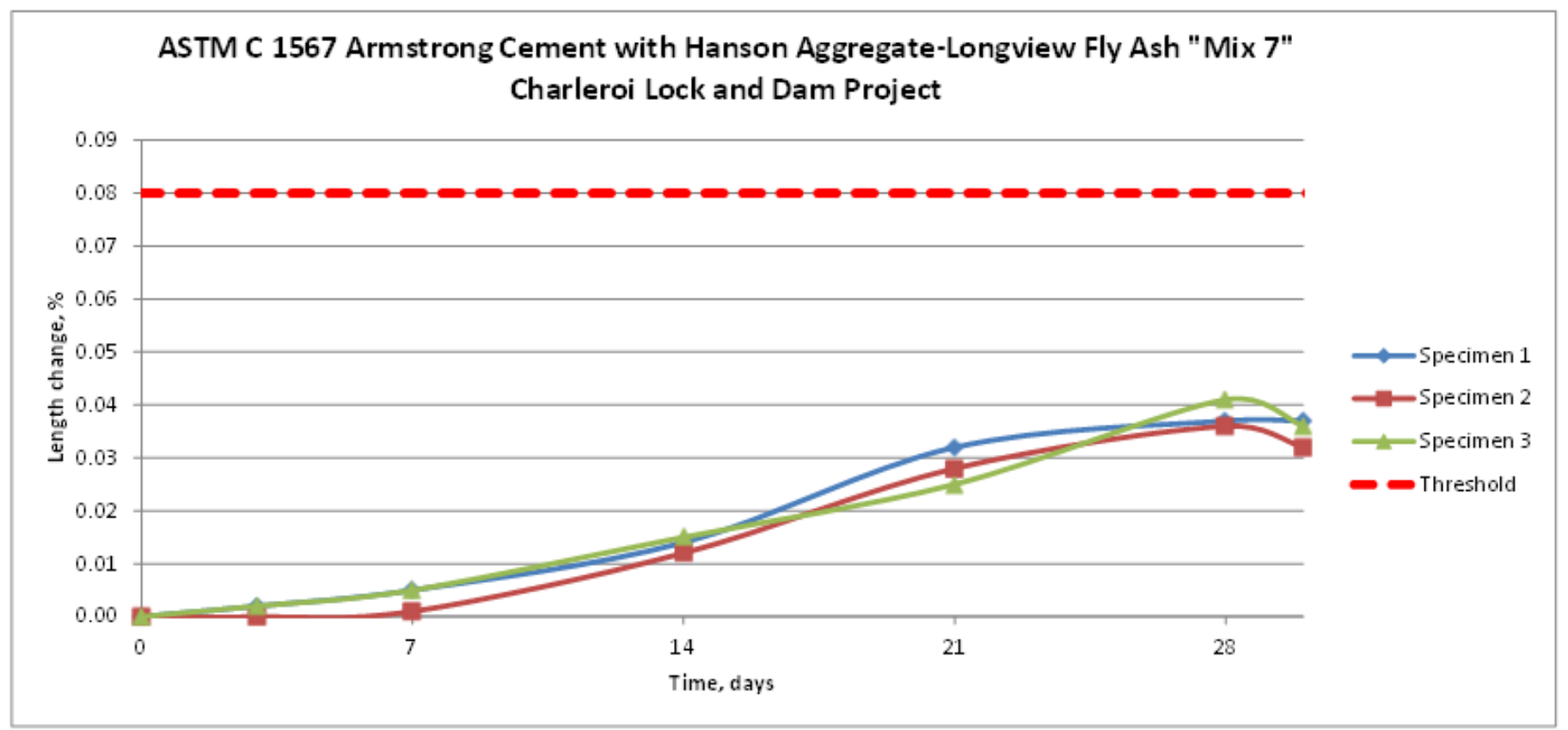




\begin{tabular}{|c|c|c|c|c|c|c|c|c|c|c|c|c|c|}
\hline \multirow{2}{*}{\begin{tabular}{|l|} 
Mix: \\
Potential A \\
\end{tabular}} & \multicolumn{5}{|c|}{ Mix 7 with Hanson and Brandon Shores Fly Ash } & & & & & & & \multirow{2}{*}{\begin{tabular}{|l|} 
\\
\end{tabular}} & \multirow[b]{3}{*}{ Standard } \\
\hline & Alkali Reacti & ty of $\mathrm{Agg}$ & regates, AS & TM C 1567 & & & & & & & & & \\
\hline & & & \multicolumn{3}{|c|}{ Specimen 1} & \multicolumn{3}{|c|}{ Specimen 2} & \multicolumn{3}{|c|}{ Specimen 3} & Average & \\
\hline & Date & Age & Reading & Change & Expansion & Reading & Change & Expansion & Reading & Change & Expansion & Expansion & Deviation \\
\hline & $\mathrm{D} / \mathrm{M} Y \mathrm{r}$ & (day) & (in.) & (in.) & $(\%)$ & (in.) & (in.) & $(\%)$ & (in.) & (in.) & $(\%)$ & $(\%)$ & $(\%)$ \\
\hline Mix & $3 / 20 / 2018$ & & - & - & - & - & - & - & - & - & - & - & - \\
\hline Initial & $3 / 22 / 2018$ & & -0.0038 & & & 0.0273 & & & 0.0112 & & & & \\
\hline \multirow[t]{7}{*}{ zero read } & $3 / 23 / 2018$ & 0 & 0.0036 & & \begin{tabular}{l|l}
0.0000 \\
\end{tabular} & 0.0330 & & 0.0000 & 0.0169 & & 0.0000 & 0.0000 & 0.0000 \\
\hline & $3 / 26 / 2018$ & 3 & 0.0039 & 0.0003 & \begin{tabular}{l|l}
0.0030 \\
\end{tabular} & 0.0332 & 0.0002 & 0.0020 & 0.0172 & 0.0003 & 0.0030 & 0.0027 & 0.0006 \\
\hline & $3 / 28 / 2018$ & 5 & 0.0041 & 0.0005 & \begin{tabular}{l|l|}
0.0050 \\
\end{tabular} & 0.0335 & 0.0005 & 0.0050 & 0.0176 & 0.0007 & 0.0070 & 0.0057 & 0.0012 \\
\hline & $3 / 30 / 2018$ & 7 & 0.0042 & 0.0006 & 0.0060 & 0.0341 & 0.0011 & 0.0110 & 0.0182 & 0.0013 & 0.0130 & 0.0100 & 0.0036 \\
\hline & $4 / 6 / 2018$ & 14 & 0.0053 & 0.0017 & \begin{tabular}{l|l}
0.0170 \\
\end{tabular} & 0.0350 & 0.0020 & 0.0200 & 0.0189 & 0.0020 & 0.0200 & 0.0190 & 0.0017 \\
\hline & $4 / 20 / 2018$ & 28 & 0.0070 & 0.0034 & 0.0340 & 0.0362 & 0.0032 & 0.0320 & 0.0201 & 0.0032 & 0.0320 & 0.0327 & 0.0012 \\
\hline & $4 / 23 / 2018$ & 30 & 0.0071 & 0.0035 & 0.0350 & 0.0363 & 0.0033 & 0.0330 & 0.0202 & 0.0033 & 0.0330 & 0.0337 & 0.0012 \\
\hline
\end{tabular}

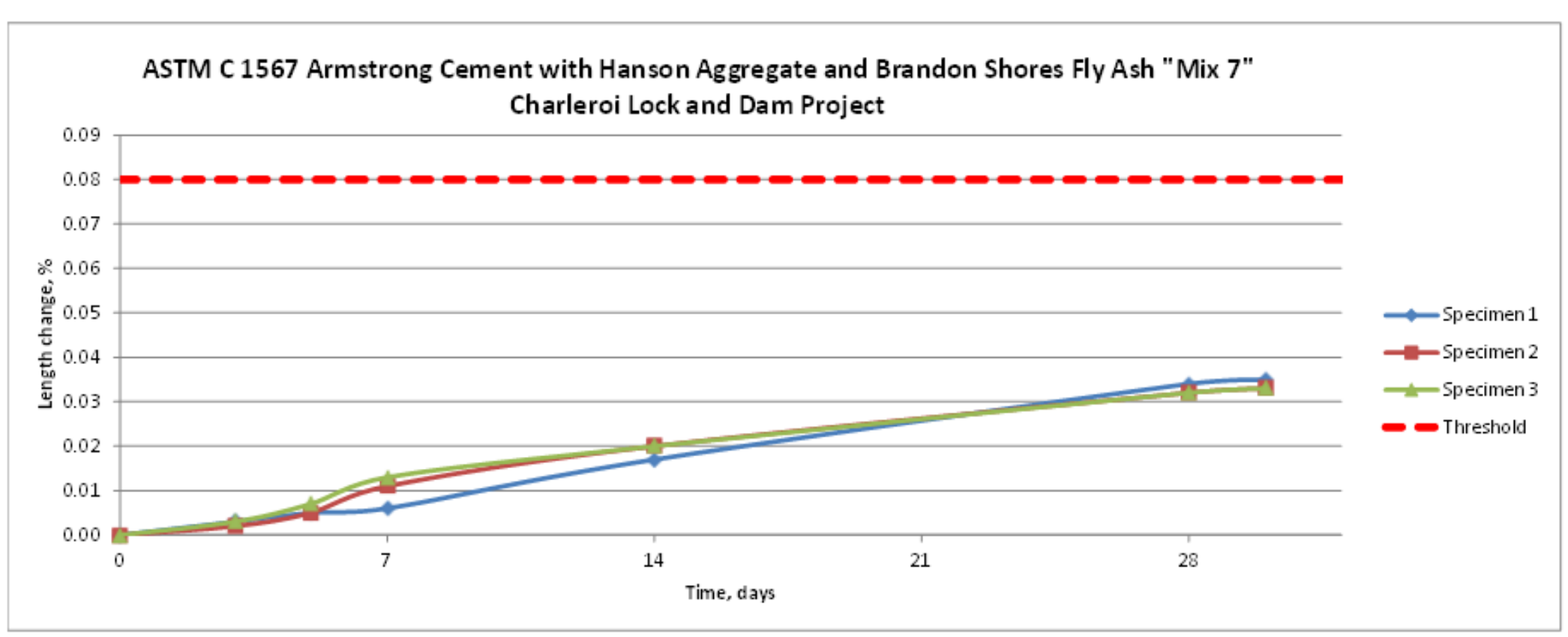




\begin{tabular}{|c|c|c|c|c|c|c|c|c|c|c|c|c|c|c|c|c|}
\hline \multirow{2}{*}{\multicolumn{17}{|c|}{\begin{tabular}{|l|l|} 
Mix: & Mix 7 with Georgetown and Sammis Fly Ash \\
Potential Alkali Reactivity of Aggregates, A STM C 1567
\end{tabular}}} \\
\hline & & & & & & & & & & & & & & & \multirow{3}{*}{\begin{tabular}{|c|} 
Average \\
Expansion \\
\end{tabular}} & \\
\hline & & & \multicolumn{3}{|c|}{ Specimen 1} & \multicolumn{3}{|c|}{ Specimen 2} & \multicolumn{3}{|c|}{ Specimen 3} & \multicolumn{3}{|c|}{ Specimen 4} & & \multirow{2}{*}{\begin{tabular}{|c|} 
Standard \\
Deviation \\
\end{tabular}} \\
\hline & Date & Age & Reading & \begin{tabular}{l|l} 
Change \\
\end{tabular} & \begin{tabular}{|l|} 
Expansion \\
\end{tabular} & Reading & $\begin{array}{l}\text { Change } \\
\end{array}$ & Expansion & Reading & \begin{tabular}{l|l} 
Change \\
\end{tabular} & Expansion & \begin{tabular}{|l|} 
Reading \\
\end{tabular} & Change & Expansion & & \\
\hline & DMYr & (day) & (in.) & (in.) & $(\%)$ & (in.) & (in.) & (\%) & (in.) & (in.) & (\%) & (in.) & (in.) & $(\%)$ & (\%) & (\%) \\
\hline Initial & $4 / 26 / 2018$ & & -0.0062 & & & -0.0039 & & & -0.0095 & & & -0.0095 & & & & \\
\hline \multirow[t]{6}{*}{ zero read } & $4 / 27 / 2018$ & 0 & 0.0007 & & 0.0000 & 0.0028 & & 0.0000 & -0.0026 & & 0.0000 & -0.0032 & & 0.0000 & 0.0000 & 0.0000 \\
\hline & $4 / 30 / 2018$ & 3 & 0.0009 & 0.0002 & 0.0020 & 0.0031 & 0.0003 & 0.0030 & -0.0023 & 0.0003 & 0.0030 & -0.0026 & 0.0006 & 0.0060 & 0.0035 & 0.0017 \\
\hline & $5 / 2 / 2018$ & 5 & 0.0010 & 0.0003 & 0.0030 & 0.0032 & 0.0004 & 0.0040 & -0.0021 & 0.0005 & 0.0050 & -0.0024 & 0.0008 & 0.0080 & 0.0050 & 0.0022 \\
\hline & $5 / 11 / 2018$ & 14 & 0.0016 & 0.0009 & 0.0090 & 0.0037 & 0.0009 & 0.0090 & -0.0016 & 0.0010 & 0.0100 & -0.0021 & 0.0011 & 0.0110 & 0.0098 & 0.0010 \\
\hline & $5 / 25 / 2018$ & 28 & 0.0018 & 0.0011 & 0.0110 & 0.0039 & 0.0011 & 0.0110 & -0.0012 & 0.0014 & 0.0140 & -0.0022 & 0.0010 & 0.0100 & 0.0115 & 0.0017 \\
\hline & $5 / 28 / 2018$ & $30 \mid$ & 0.0020 & 0.0013 & $\mid 0.0130$ & 0.0040 & 0.0012 & 0.0120 & -0.0011 & 0.0015 & 0.0150 & -0.0020 & 0.0012 & 0.0120 & 0.0130 & 0.0014 \\
\hline
\end{tabular}

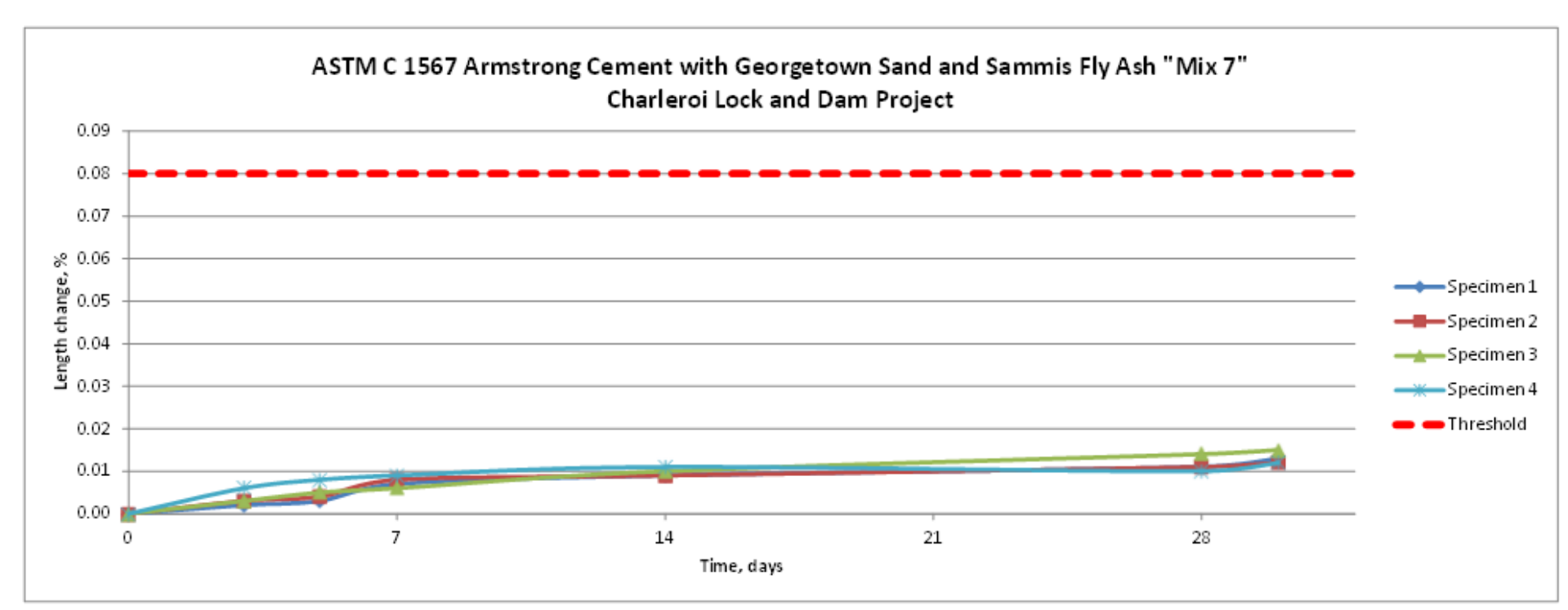




\begin{tabular}{|c|c|c|c|c|c|c|c|c|c|c|c|c|c|c|c|c|}
\hline \multirow{2}{*}{\multicolumn{17}{|c|}{\begin{tabular}{|l|l|} 
Mix: & Mix 7 with Georgetown and Ft. Martin Fly Ash \\
Potential Alkali Reactivity of Aggregates, ASTM C 1567 \\
\end{tabular}}} \\
\hline \multicolumn{15}{|c|}{ Potential Alkali Reactivity of Aggregates, A STM C 1567} & & \\
\hline & & & \multicolumn{3}{|c|}{ Specimen 1} & \multicolumn{3}{|c|}{ Specimen 2} & \multicolumn{3}{|c|}{ Specimen 3} & \multicolumn{3}{|c|}{ Specimen 4} & Average & Standard \\
\hline & Date & Age & Reading & Change & Expansion & Reading & Change & Expansion & Reading & Change & Expansion & Reading & Change & Expansion & Expansion & Deviation \\
\hline & DMYr & (day) & (in.) & (in.) & $(\%)$ & (in.) & (in.) & $(\%)$ & (in.) & (in.) & $(\%)$ & (in.) & (in.) & $(\%)$ & $(\%)$ & (\%) \\
\hline Mix & $5 / 1 / 2018$ & & - & - & - & - & - & - & - & - & - & - & - & - & - & - \\
\hline Initial & $5 / 3 / 2018$ & & -0.0128 & & & -0.0039 & & & 0.0017 & & & -0.0099 & & & & \\
\hline zero read & $5 / 4 / 2018$ & 0 & -0.0059 & & 0.0000 & $\begin{array}{ll}0.0029 \\
\end{array}$ & & 0.0000 & 0.0090 & & 0.0000 & -0.0030 & & 0.0000 & 0.0000 & 0.0000 \\
\hline & $5 / 7 / 2018$ & 3 & -0.0055 & 0.0004 & 0.0040 & 0.0032 & 0.0003 & 0.0030 & 0.0092 & 0.0002 & 0.0020 & -0.0029 & 0.0001 & 0.0010 & 0.0025 & 0.0013 \\
\hline & $5 / 9 / 2018$ & 5 & -0.0050 & 0.0009 & 0.0090 & 0.0037 & 0.0008 & \begin{tabular}{l|l|}
0.0080 \\
\end{tabular} & $\begin{array}{l}0.0097 \\
\end{array}$ & 0.0007 & $\begin{array}{l}0.0070 \\
\end{array}$ & -0.0024 & 0.0006 & 0.0060 & 0.0075 & 0.0013 \\
\hline & $5 / 11 / 2018$ & 7 & -0.0049 & 0.0010 & 0.0100 & 0.0040 & 0.0011 & 0.0110 & 0.0099 & 0.0009 & 0.0090 & -0.0022 & 0.0008 & 0.0080 & 0.0095 & 0.0013 \\
\hline & $5 / 18 / 2018$ & 14 & -0.0048 & 0.0011 & 0.0110 & 0.0044 & 0.0015 & 0.0150 & 0.0100 & 0.0010 & 0.0100 & -0.0015 & 0.0015 & 0.0150 & 0.0128 & 0.0026 \\
\hline & $6 / 1 / 2018$ & 28 & -0.0046 & 0.0013 & 0.0130 & 0.0047 & 0.0018 & 0.0180 & 0.0101 & 0.0011 & \begin{tabular}{|l|l|}
0.0110 \\
\end{tabular} & -0.0013 & 0.0017 & 0.0170 & 0.0148 & 0.0033 \\
\hline & $6 / 4 / 2018$ & $31 \mid$ & -0.0041 & 0.0018 & 0.0180 & 0.0047 & 0.0018 & 0.0180 & 0.0103 & 0.0013 & 0.0130 & -0.0012 & 0.0018 & 0.0180 & 0.0168 & 0.0025 \\
\hline
\end{tabular}

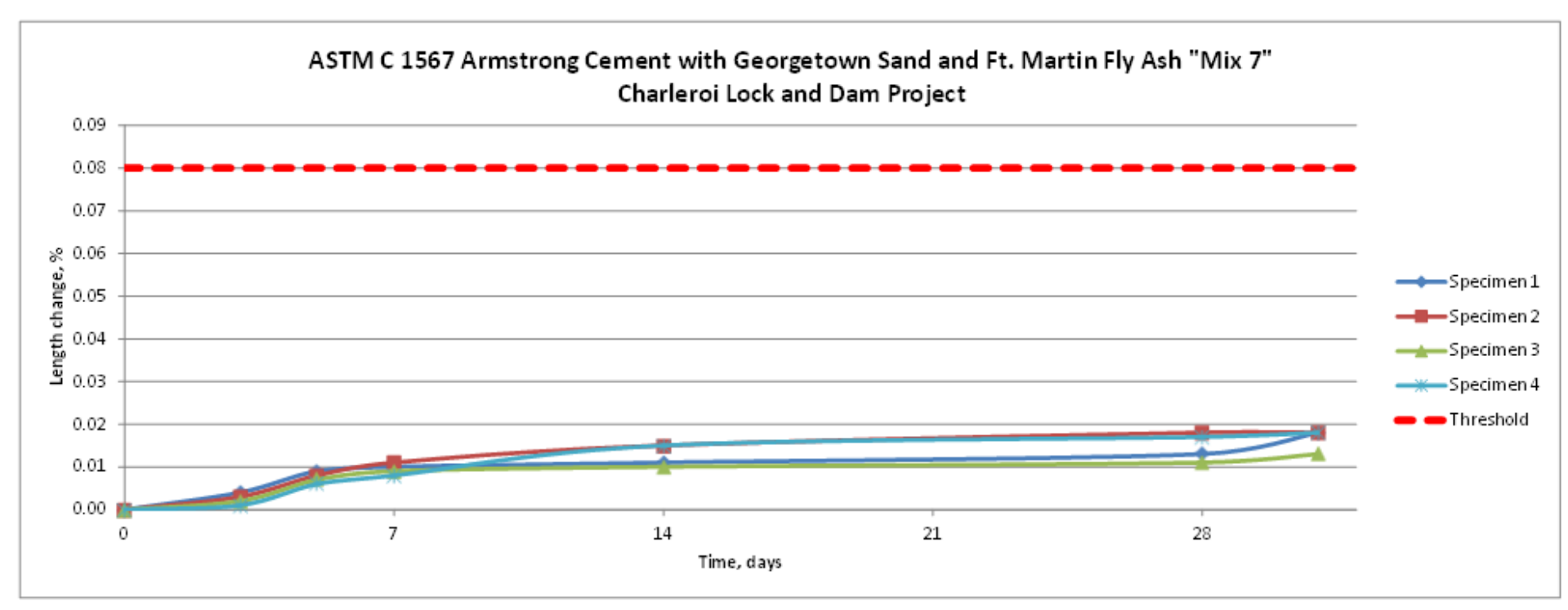




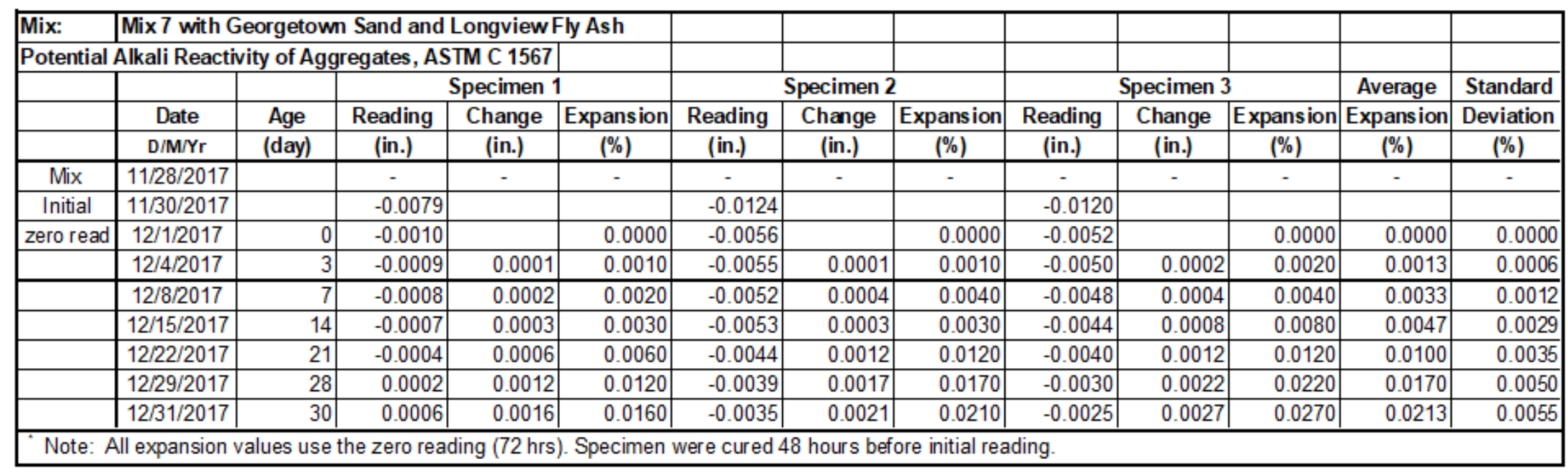

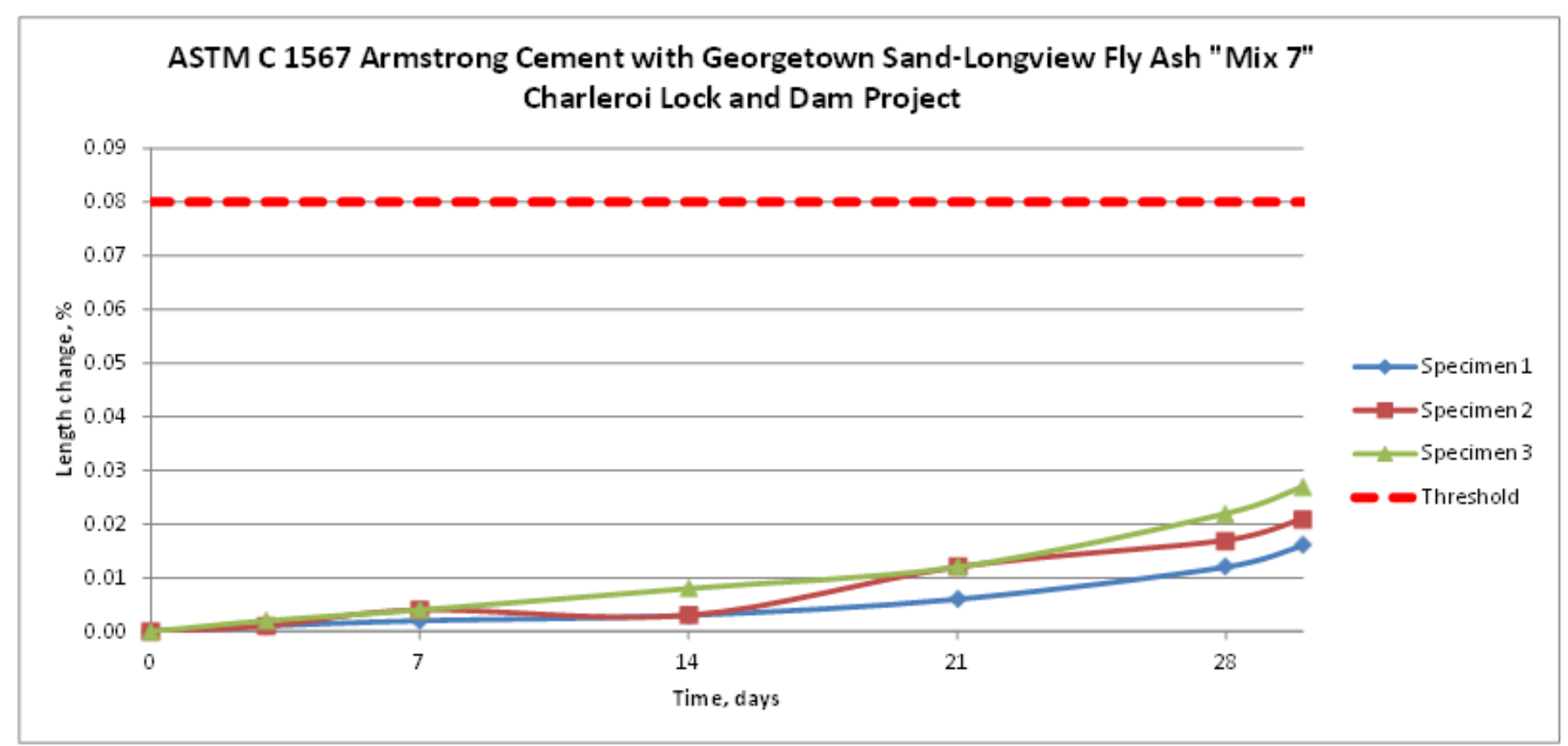




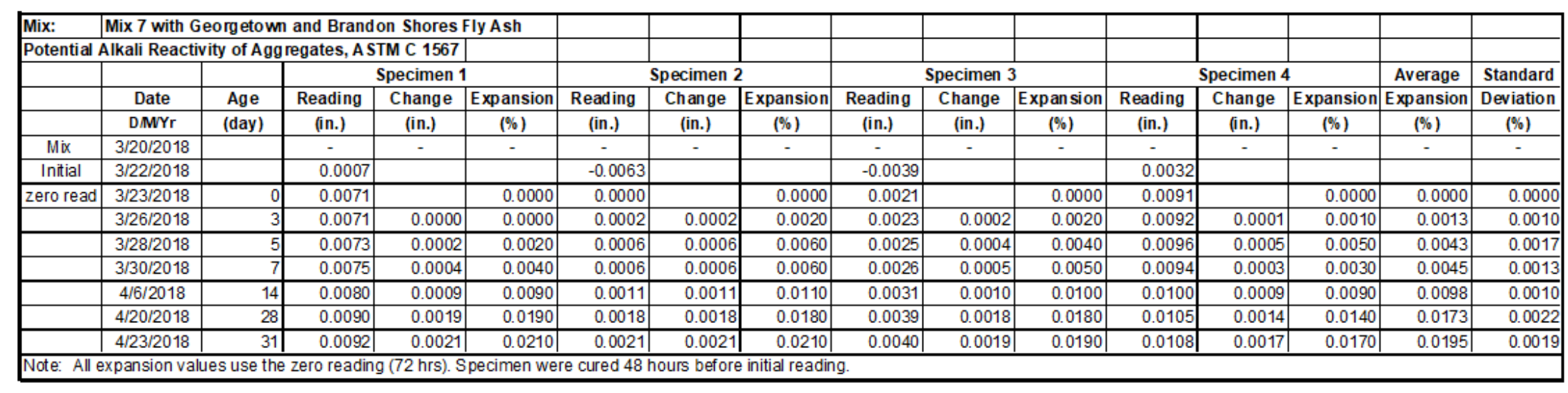

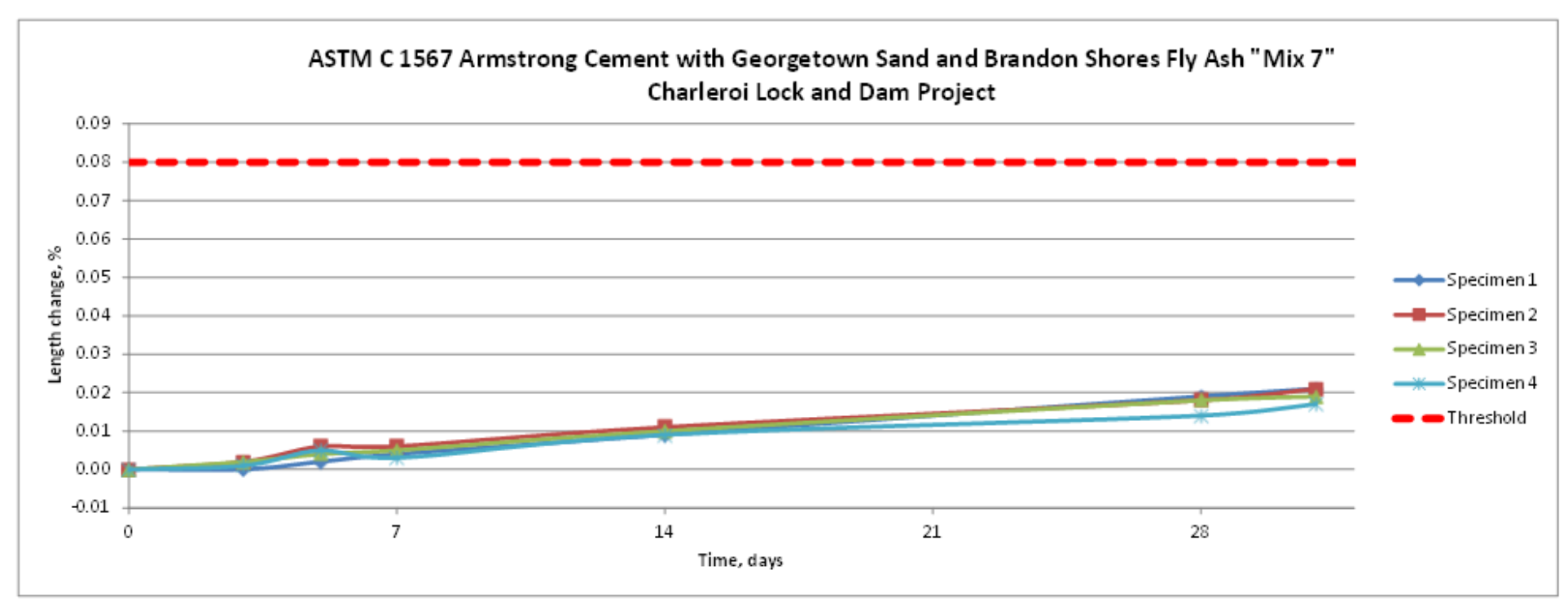




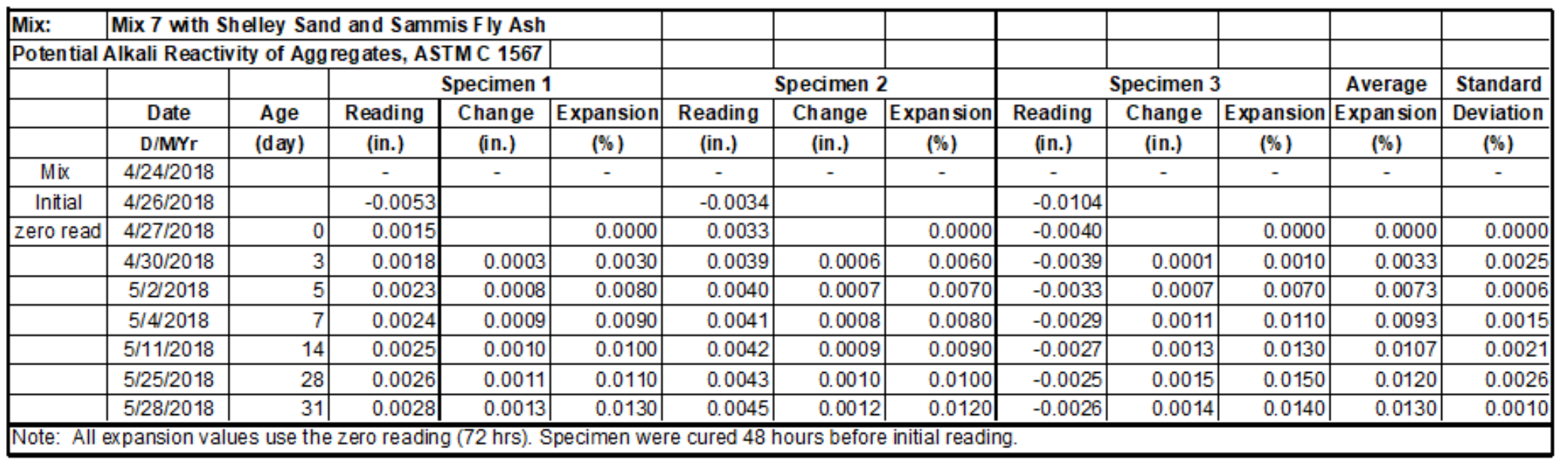

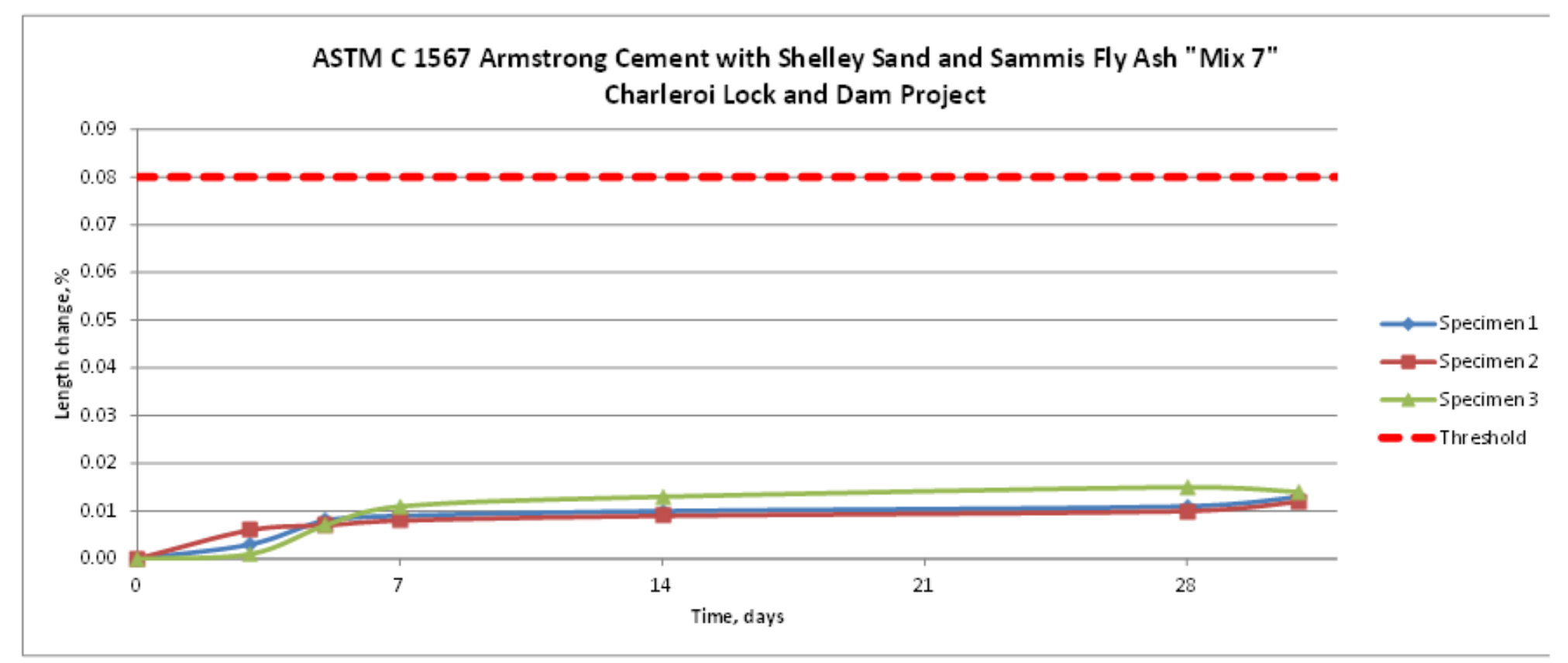




\begin{tabular}{|c|c|c|c|c|c|c|c|c|c|c|c|c|c|c|c|c|}
\hline Mix: & \multicolumn{5}{|c|}{ Mix 7 with Shelley Sand and F t. Martin Fly Ash } & & & & & & & & & & & \\
\hline \multicolumn{17}{|c|}{\begin{tabular}{|l|l|l|l|} 
Potential Alkali Reactivity of Agg regates, A STM C 1567 & \\
\end{tabular}} \\
\hline & & & \multicolumn{3}{|c|}{ Specimen 1} & \multicolumn{3}{|c|}{ Specimen 2} & \multicolumn{3}{|c|}{ Specimen 3} & \multicolumn{3}{|c|}{ Specimen 4} & Average & Standard \\
\hline & Date & Age & Reading & Change & Expansion & Reading & Change & \begin{tabular}{|l|} 
Expansion \\
\end{tabular} & \begin{tabular}{l|} 
Reading \\
\end{tabular} & Change & Expansion & Reading & Change & Expansion & Expansion & Deviation \\
\hline & D/WYr & (day) & (in.) & (in.) & $(\%)$ & (in.) & (in.) & $(\%)$ & (in.) & (in.) & (\%) & (in.) & (in.) & $(\%)$ & $(\%)$ & (\%) \\
\hline$M i x$ & $5 / 1 / 2018$ & & - & - & - & - & - & - & - & - & - & - & - & - & - & - \\
\hline Initial & $5 / 3 / 2018$ & & -0.0106 & & & -0.0106 & & & -0.0096 & & & -0.0052 & & & & \\
\hline \multirow[t]{6}{*}{ zero read } & $5 / 4 / 2018$ & 0 & -0.0033 & & 0.0000 & -0.0037 & & $\begin{array}{l}0.0000 \\
\end{array}$ & -0.0026 & & 0.0000 & 0.0021 & & 0.0000 & 0.0000 & 0.0000 \\
\hline & $5 / 7 / 2018$ & 3 & -0.0030 & 0.0003 & 0.0030 & -0.0034 & 0.0003 & 0.0030 & -0.0023 & 0.0003 & 0.0030 & 0.0021 & 0.0000 & 0.0000 & 0.0023 & 0.0015 \\
\hline & $5 / 9 / 2018$ & 5 & -0.0027 & 0.0006 & $\begin{array}{ll}0.0060 \\
\end{array}$ & -0.0030 & 0.0007 & 0.0070 & -0.0022 & 0.0004 & 0.0040 & 0.0025 & 0.0004 & 0.0040 & 0.0053 & 0.0015 \\
\hline & $5 / 18 / 2018$ & 14 & -0.0022 & 0.0011 & \begin{tabular}{l|l|}
0.0110 \\
\end{tabular} & -0.0024 & 0.0013 & $\begin{array}{l}0.0130 \\
\end{array}$ & -0.0018 & 0.0008 & 0.0080 & 0.0030 & 0.0009 & 0.0090 & 0.0103 & 0.0022 \\
\hline & $6 / 1 / 2018$ & 28 & -0.0018 & 0.0015 & $\begin{array}{ll}0.0150 \\
\end{array}$ & -0.0018 & 0.0019 & $\begin{array}{l}0.0190 \\
\end{array}$ & -0.0019 & 0.0007 & 0.0070 & 0.0033 & 0.0012 & 0.0120 & 0.0133 & 0.0051 \\
\hline & $6 / 4 / 2018$ & $31 \mid$ & -0.0013 & 0.0020 & 0.0200 & -0.0019 & 0.0018 & 0.0180 & -0.0015 & 0.0011 & 0.0110 & 0.0036 & 0.0015 & 0.0150 & 0.0160 & 0.0039 \\
\hline
\end{tabular}

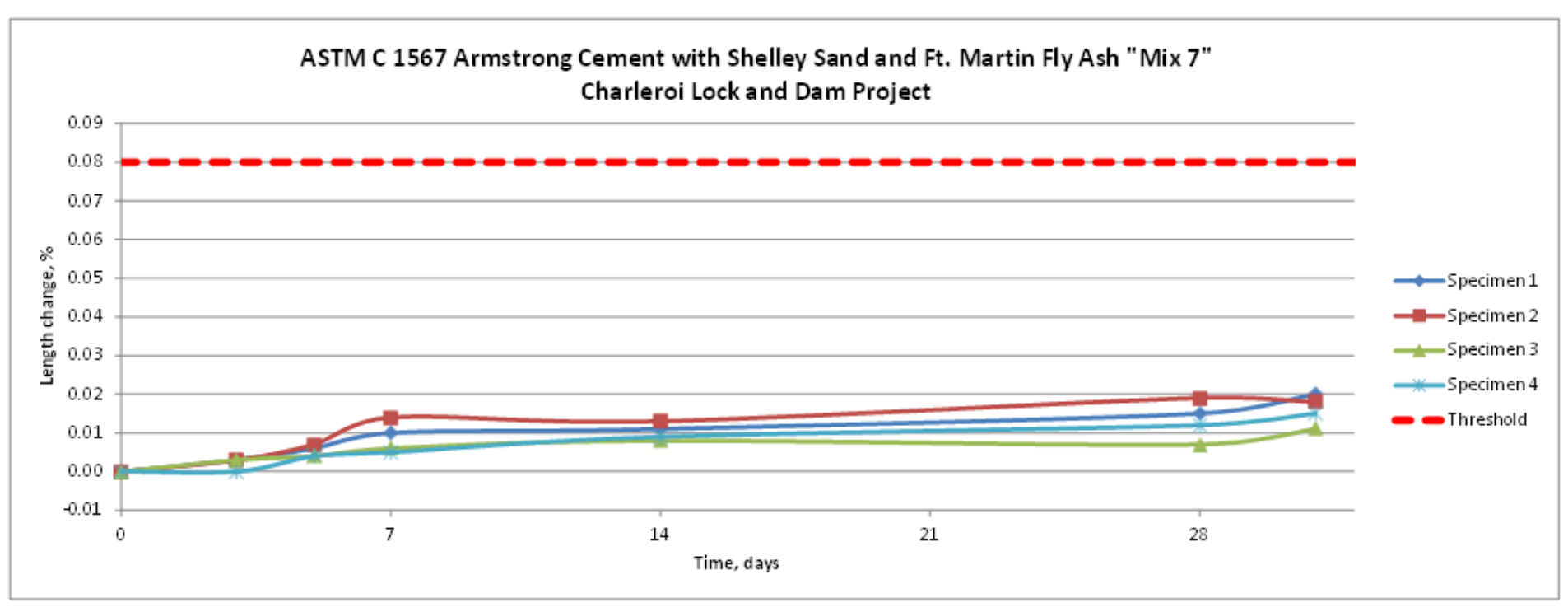




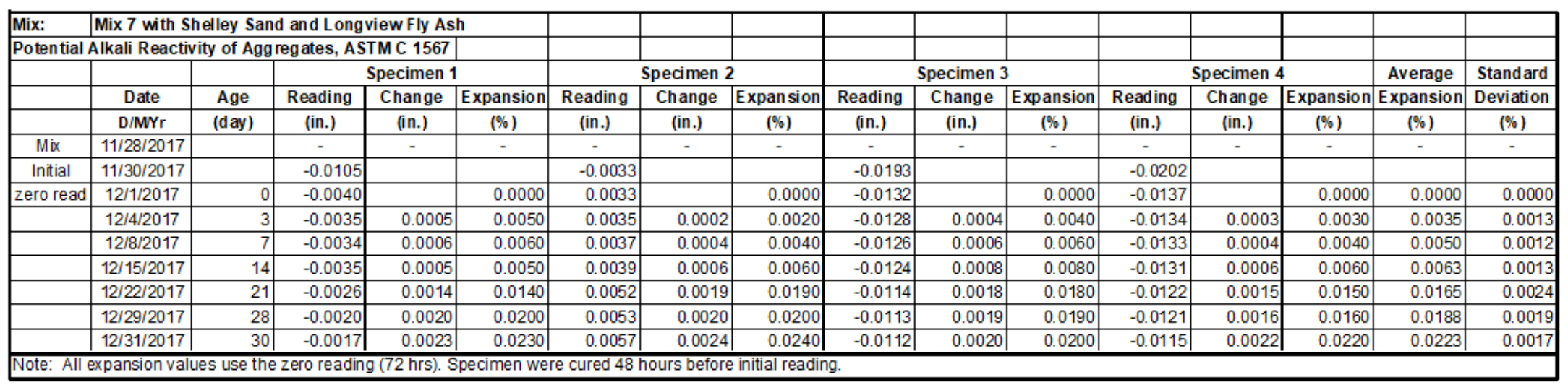

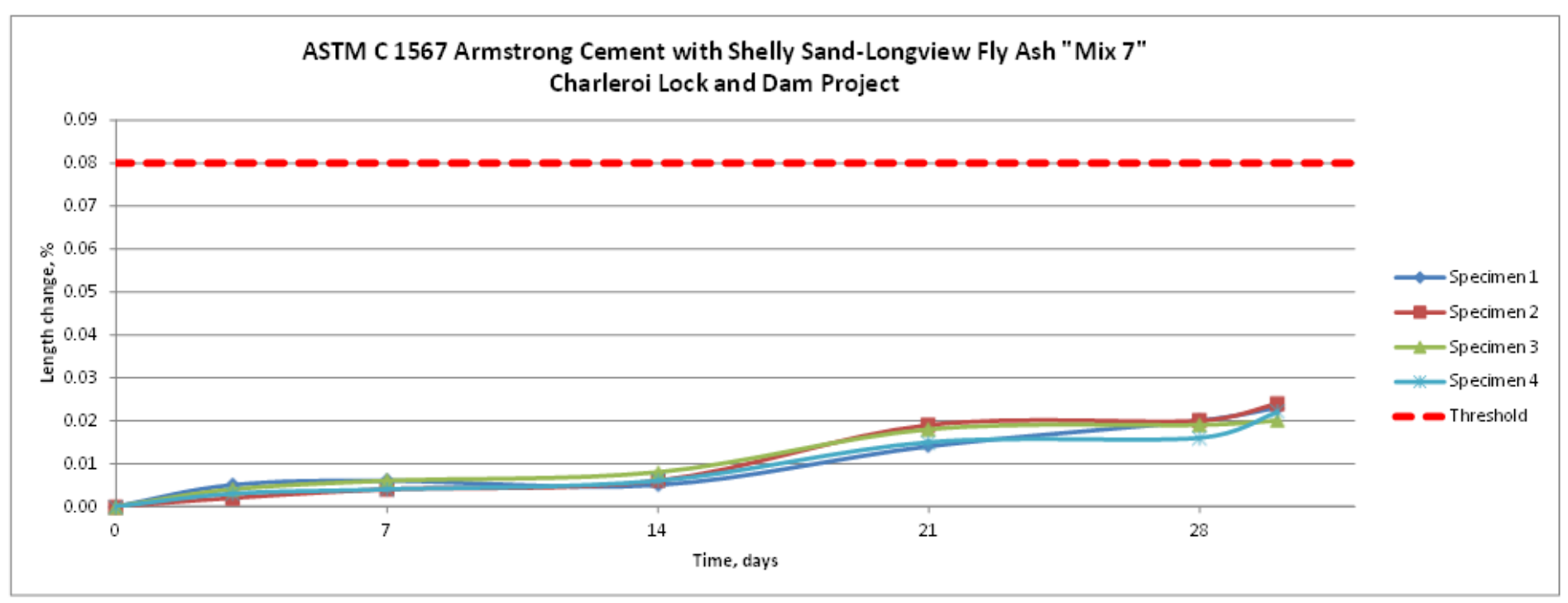




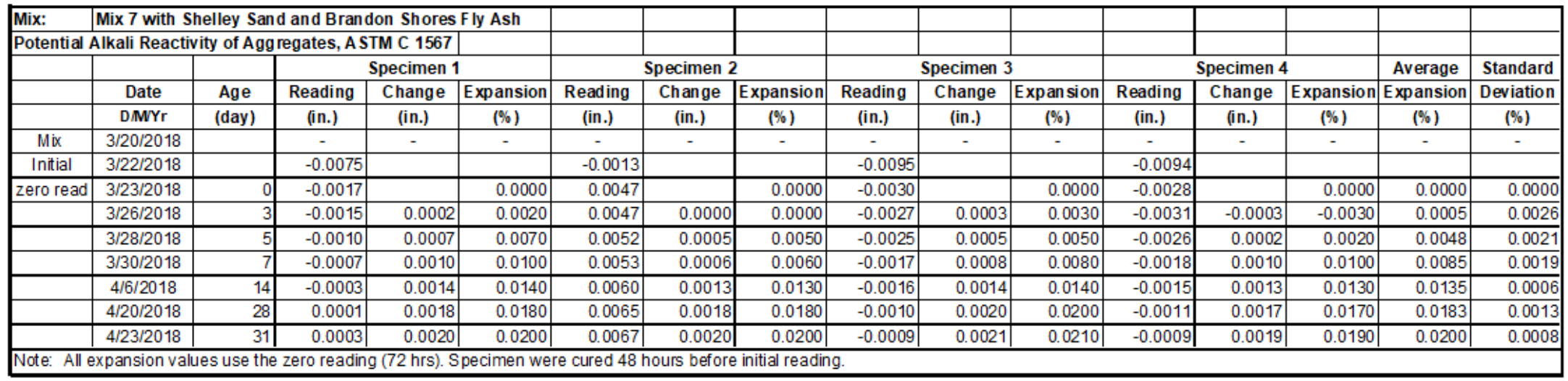

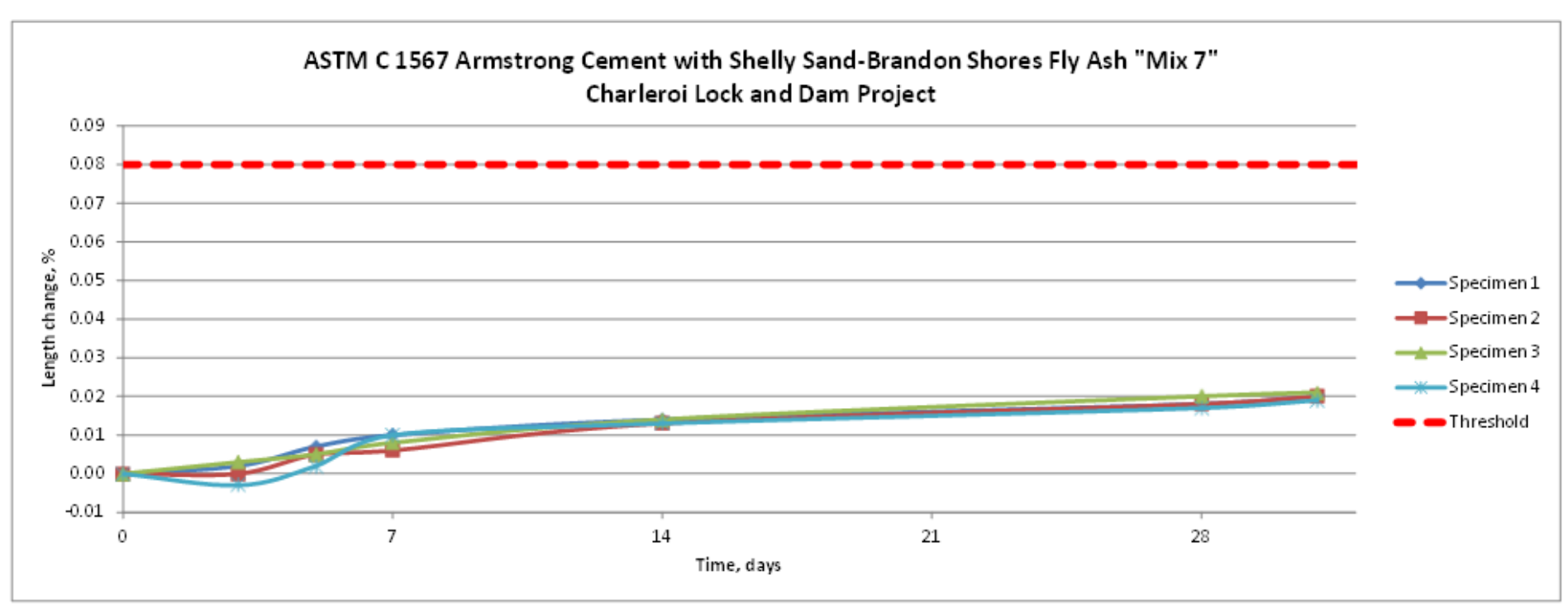




\begin{tabular}{|c|c|c|c|c|c|c|c|c|c|c|c|c|c|c|c|c|}
\hline Mix: & \multicolumn{5}{|c|}{ Mix 10 with Hanson Aggregate and Sammis Fly Ash } & & & & & & & & & & & \\
\hline \multicolumn{17}{|c|}{ Potential Alkali Reactivity of Aggregates, ASTM C 1567 } \\
\hline & Date & & \multicolumn{3}{|c|}{ Specimen 1} & \multicolumn{3}{|c|}{ Specimen 2} & \multicolumn{3}{|c|}{ Specimen 3} & \multicolumn{3}{|c|}{ Specimen 4} & Average & Standard \\
\hline Mixture & & Age $^{*}$ & Reading & Change & Expansior & Reading & Change & Expansior & Reading & Change & Expansion & Reading & Change & Expansion & Expansior & Deviation \\
\hline ID & $\mathrm{D} / \mathrm{M} Y \mathrm{r}$ & (day) & (in.) & (in.) & $(\%)$ & (in.) & (in.) & $(\%)$ & (in.) & (in.) & $(\%)$ & (in.) & (in.) & $(\%)$ & $(\%)$ & $(\%)$ \\
\hline Mix & $10 / 11 / 2016$ & & - & - & - & - & - & - & - & - & - & - & - & - & - & - \\
\hline Initial & $10 / 13 / 2016$ & & -0.0070 & & & -0.0058 & & & -0.0029 & & & 0.0032 & & & & \\
\hline \multirow[t]{7}{*}{ zero read } & $10 / 14 / 2016$ & 0 & -0.0004 & & 0.0000 & 0.0010 & & 0.0000 & 0.0038 & & 0.0000 & 0.0101 & & & 0.0000 & 0.0000 \\
\hline & 10/19/2016 & 5 & 0.0010 & 0.0014 & 0.0140 & 0.0022 & 0.0012 & 0.0120 & 0.0051 & 0.0013 & 0.0130 & 0.0110 & 0.0009 & 0.0090 & 0.0120 & 0.0022 \\
\hline & $10 / 21 / 2016$ & 7 & 0.0013 & 0.0017 & 0.0170 & 0.0024 & 0.0014 & 0.0140 & 0.0052 & 0.0014 & 0.0140 & 0.0111 & 0.0010 & \begin{tabular}{l|l}
0.0100 \\
\end{tabular} & 0.0138 & 0.0029 \\
\hline & $10 / 28 / 2016$ & 14 & 0.0023 & 0.0027 & 0.0270 & 0.0046 & 0.0036 & 0.0360 & 0.0066 & 0.0028 & 0.0280 & 0.0130 & 0.0029 & 0.0290 & 0.0300 & 0.0041 \\
\hline & $11 / 4 / 2016$ & 21 & 0.0053 & 0.0057 & 0.0570 & 0.0064 & 0.0054 & 0.0540 & 0.0090 & 0.0052 & 0.0520 & 0.0154 & 0.0053 & 0.0530 & 0.0540 & 0.0022 \\
\hline & $11 / 11 / 2016$ & 28 & 0.0060 & 0.0064 & 0.0640 & 0.0068 & 0.0058 & 0.0580 & 0.0100 & 0.0062 & 0.0620 & 0.0159 & 0.0058 & 0.0580 & 0.0605 & 0.0030 \\
\hline & $11 / 13 / 2016$ & 30 & 0.0061 & 0.0065 & 0.0650 & \begin{tabular}{l|l|}
0.0070 \\
\end{tabular} & 0.0060 & 0.0600 & 0.0102 & 0.0064 & 0.0640 & \begin{tabular}{|c|}
0.0167 \\
\end{tabular} & 0.0066 & 0.0660 & 0.0638 & 0.0026 \\
\hline
\end{tabular}

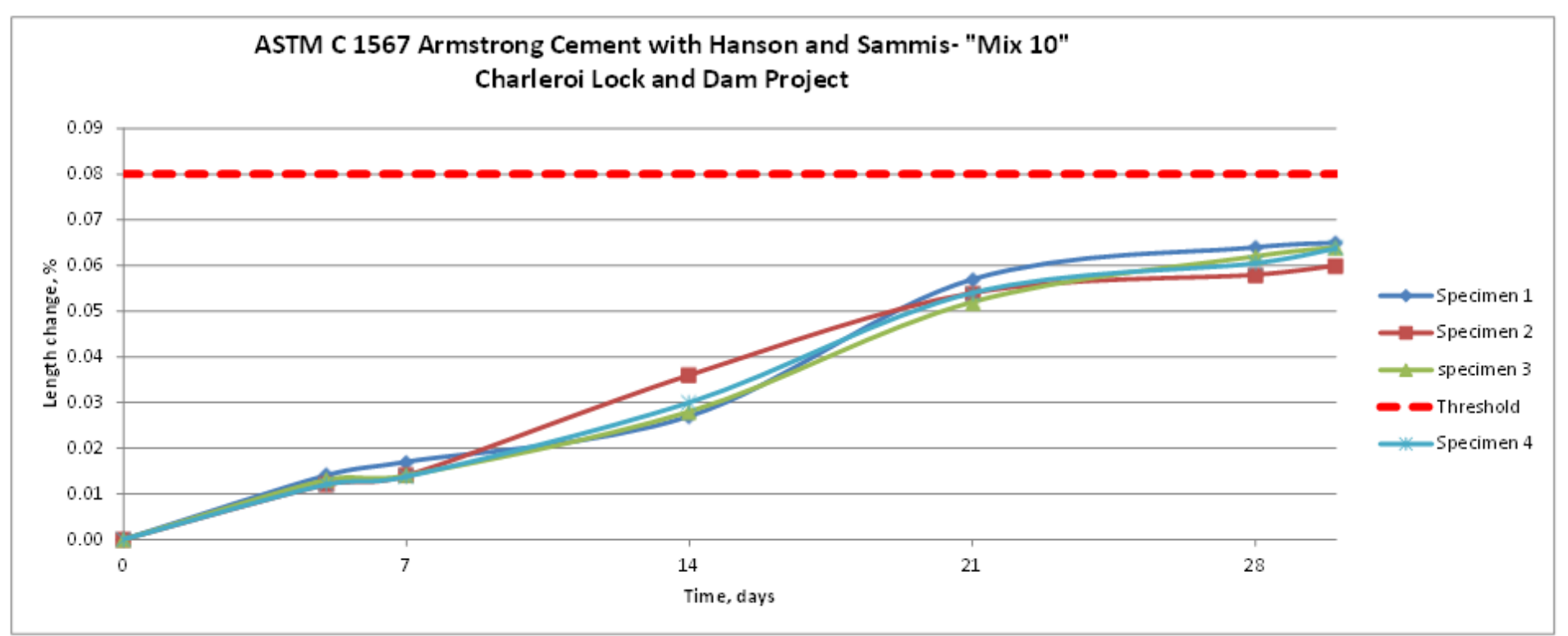




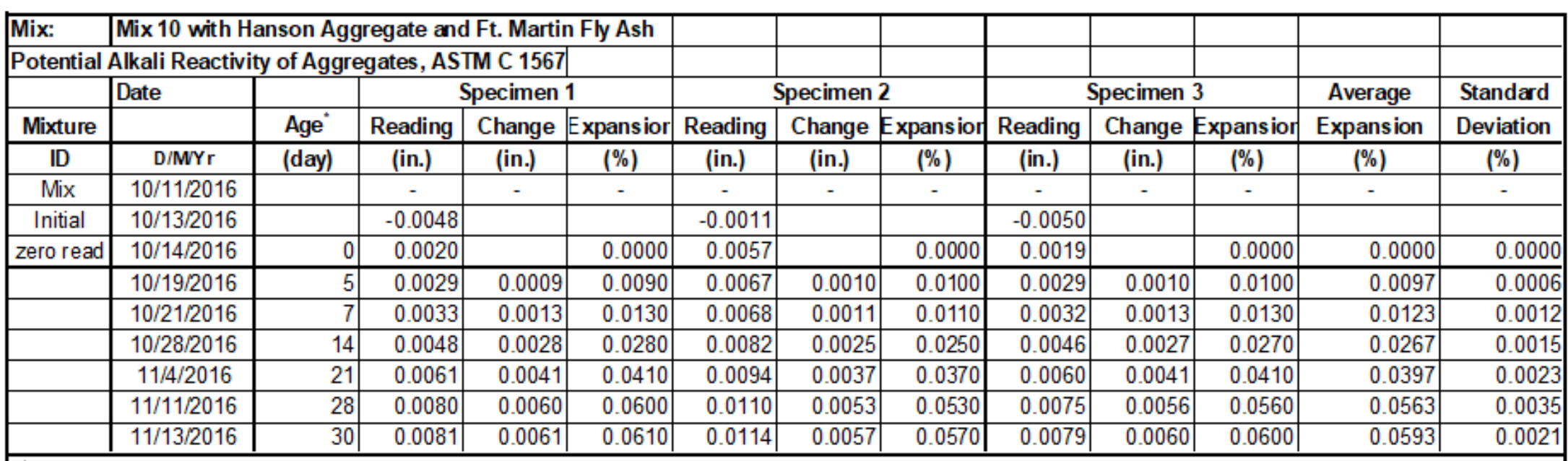

Note: All expansion values use the zero reading ( $72 \mathrm{hrs}$ ). Specimen were cured 48 hours before initial reading. Age (day) represents time of measurement after casting.

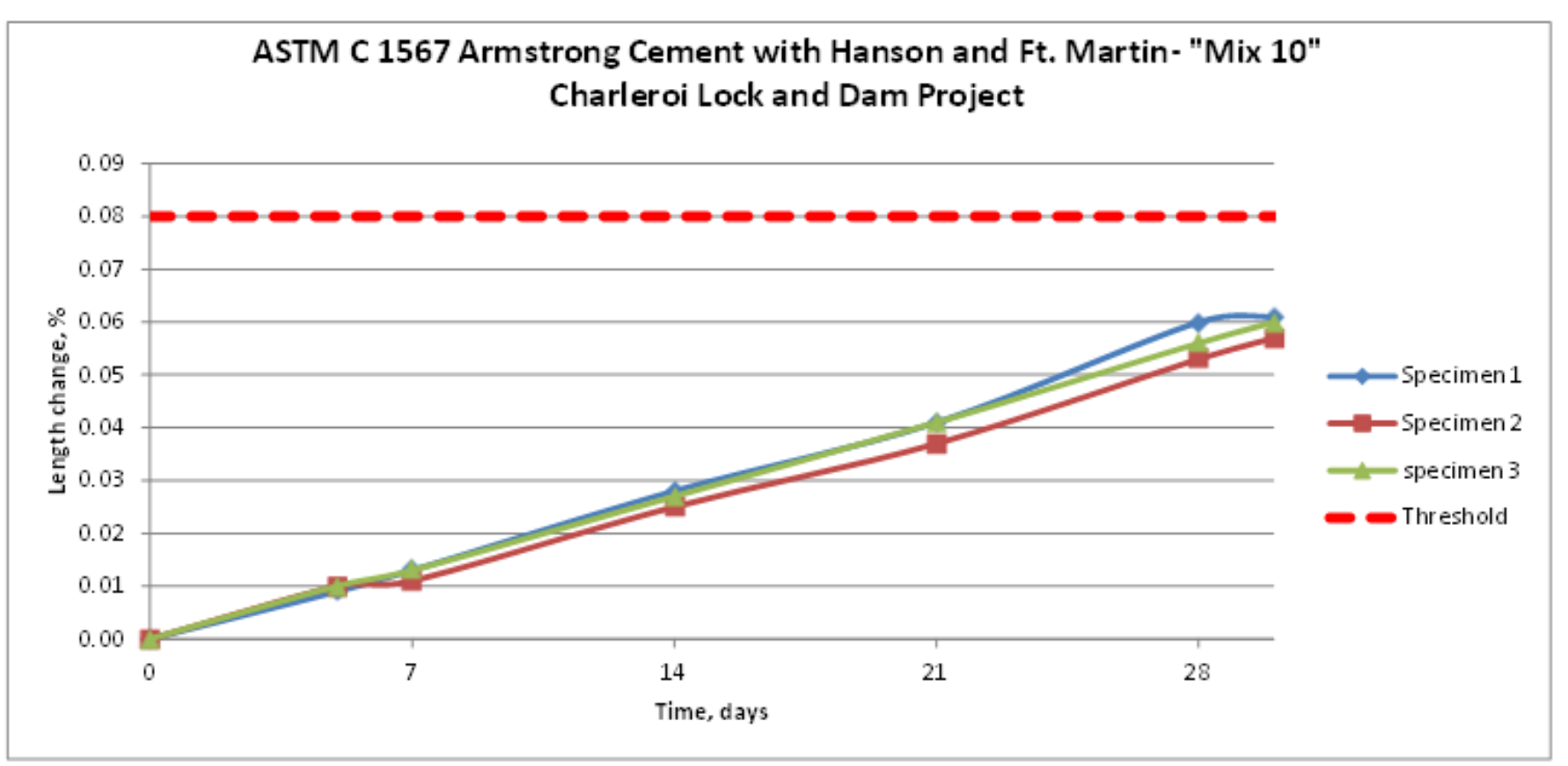




\begin{tabular}{|c|c|c|c|c|c|c|c|c|c|c|c|c|c|c|c|c|}
\hline \multicolumn{17}{|c|}{\begin{tabular}{|l|l|} 
Mix: & Mix 10 with Han son and Longview F ly Ash \\
\end{tabular}} \\
\hline \multicolumn{17}{|c|}{ Potential Alkali Reactivity of Aggregates, A STM C 1567} \\
\hline & & & \multicolumn{3}{|c|}{ Specimen 1} & \multicolumn{3}{|c|}{ Specimen 2} & \multicolumn{3}{|c|}{ Specimen 3} & \multicolumn{3}{|c|}{ Specimen 4} & Average & Standard \\
\hline & Date & Age & Reading & Change & Expansion & Reading & Change & Expansion & Reading & Change & Expansion & Reading & Change & Expansion & Expansion & Deviation \\
\hline & DMYr & (day) & (in.) & (in.) & (\%) & (in.) & (in.) & $(\%)$ & (in.) & (in.) & (\%) & (in.) & (in.) & (\%) & (\%) & (\%) \\
\hline Mix & $12 / 5 / 2017$ & & - & - & - & - & - & - & - & - & - & - & - & - & - & - \\
\hline Initial & $12 / 7 / 2017$ & & -0.0056 & & & -0.0089 & & & -0.0087 & & & -0.0061 & & & & \\
\hline zero read & $12 / 8 / 2017$ & 0 & 0.0012 & & 0.0000 & -0.0017 & & 0.0000 & -0.0015 & & 0.0000 & 0.0013 & & 0.0000 & 0.0000 & 0.0000 \\
\hline & $12 / 11 / 2017$ & 3 & 0.0019 & 0.0007 & 0.0070 & -0.0011 & 0.0006 & 0.0060 & -0.0010 & 0.0005 & 0.0050 & 0.0019 & 0.0006 & 0.0060 & 0.0060 & 0.0008 \\
\hline & $12 / 15 / 2017$ & 7 & 0.0023 & 0.0011 & 0.0110 & -0.0004 & 0.0013 & 0.0130 & -0.0004 & 0.0011 & 0.0110 & 0.0023 & 0.0010 & 0.0100 & 0.0113 & 0.0013 \\
\hline & $12 / 22 / 2017$ & 14 & 0.0037 & 0.0025 & 0.0250 & 0.0011 & 0.0028 & 0.0280 & 0.0017 & 0.0032 & 0.0320 & 0.0044 & 0.0031 & 0.0310 & 0.0290 & 0.0032 \\
\hline & $12 / 29 / 2017$ & 21 & 0.0054 & 0.0042 & 0.0420 & 0.0020 & 0.0037 & 0.0370 & 0.0024 & 0.0039 & 0.0390 & 0.0058 & 0.0045 & 0.0450 & 0.0408 & 0.0035 \\
\hline & $1 / 5 / 2018$ & 28 & 0.0078 & 0.0066 & 0.0660 & 0.0046 & 0.0063 & 0.0630 & 0.0054 & 0.0069 & 0.0690 & 0.0081 & 0.0068 & 0.0680 & 0.0665 & 0.0026 \\
\hline & $1 / 8 / 2018$ & 30 & 0.0084 & 0.0072 & 0.0720 & 0.0054 & 0.0071 & 0.0710 & 0.0061 & 0.0076 & 0.0760 & 0.0085 & 0.0072 & 0.0720 & 0.0728 & 0.0022 \\
\hline
\end{tabular}

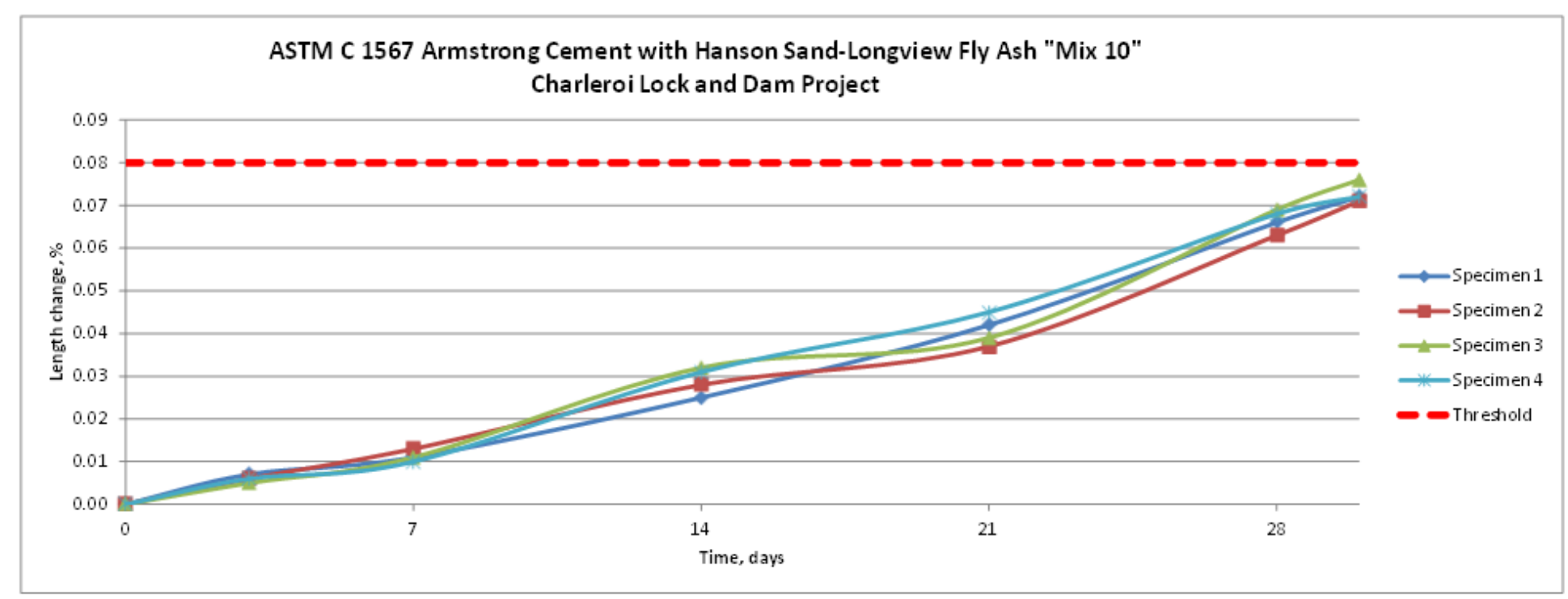




\begin{tabular}{|c|c|c|c|c|c|c|c|c|c|c|c|c|c|c|c|c|}
\hline Mix: & \multicolumn{5}{|c|}{ Mix 10 with Han sen and Brandon Shores Fly Ash } & & & & & & & & & & & \\
\hline \multicolumn{17}{|c|}{ Potential Alkali Reactivity of Aggregates, ASTM C 1567} \\
\hline & & & \multicolumn{3}{|c|}{ Specimen 1} & \multicolumn{3}{|c|}{ Specimen 2} & \multicolumn{3}{|c|}{ Specimen 3} & \multicolumn{3}{|c|}{ Specimen 4} & Average & Stand ard \\
\hline & Date & Age & Reading & Change & Expansion & Reading & Change & Expansion & Reading & Change & Expansion & Reading & Change & Expansion & Expansion & Deviation \\
\hline & D/MYr & (day) & (in.) & (in.) & $(\%)$ & (in.) & (in.) & (\%) & (in.) & (in.) & $(\%)$ & (in.) & (in.) & $(\%)$ & $(\%)$ & $(\%)$ \\
\hline Mix & $3 / 13 / 2018$ & & - & - & - & - & - & - & - & - & - & - & - & - & - & - \\
\hline Initial & $3 / 15 / 2018$ & & -0.0044 & & & -0.0091 & & & -0.0018 & & & -0.0021 & & & & \\
\hline zero read & $3 / 16 / 2018$ & 0 & 0.0024 & & 0.0000 & -0.0043 & & 0.0000 & 0.0050 & & 0.0000 & 0.0047 & & 0.0000 & 0.0000 & 0.0000 \\
\hline & $3 / 19 / 2018$ & \begin{tabular}{l|l}
3 & \\
\end{tabular} & 0.0025 & 0.0001 & 0.0010 & -0.0035 & 0.0008 & 0.0080 & 0.0052 & 0.0002 & 0.0020 & 0.0047 & 0.0000 & 0.0000 & 0.0028 & 0.0036 \\
\hline & $3 / 21 / 2018$ & 5 & $\begin{array}{l}0.0028 \\
\end{array}$ & 0.0004 & 0.0040 & -0.0033 & 0.0010 & 0.0100 & 0.0056 & 0.0006 & 0.0060 & 0.0048 & 0.0001 & 0.0010 & 0.0053 & 0.0038 \\
\hline & $3 / 23 / 2018$ & 7 & 0.0040 & 0.0016 & 0.0160 & -0.0030 & 0.0013 & 0.0130 & 0.0066 & 0.0016 & 0.0160 & 0.0055 & 0.0008 & 0.0080 & 0.0133 & 0.0038 \\
\hline & $3 / 30 / 2018$ & 14 & 0.0046 & 0.0022 & 0.0220 & -0.0012 & 0.0031 & 0.0310 & 0.0083 & 0.0033 & 0.0330 & 0.0070 & 0.0023 & 0.0230 & 0.0273 & 0.0056 \\
\hline & 4/13/2018 & 28 & 0.0079 & 0.0055 & 0.0550 & 0.0015 & 0.0058 & 0.0580 & 0.0107 & 0.0057 & 0.0570 & 0.0103 & 0.0056 & 0.0560 & 0.0565 & 0.0013 \\
\hline & $4 / 15 / 2018$ & 30 & 0.0082 & 0.0058 & 0.0580 & 0.0018 & 0.0061 & 0.0610 & 0.0110 & 0.0060 & 0.0600 & 0.0105 & 0.0058 & 0.0580 & 0.0593 & 0.0015 \\
\hline
\end{tabular}

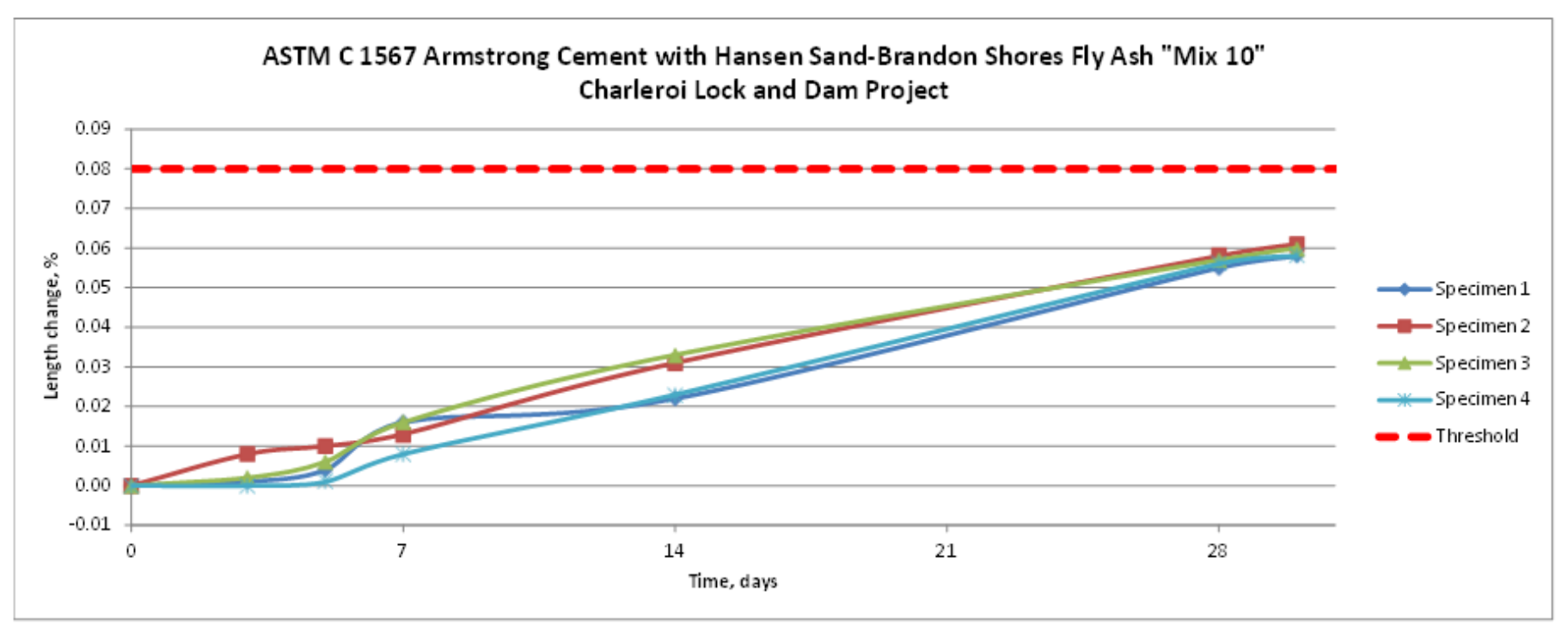




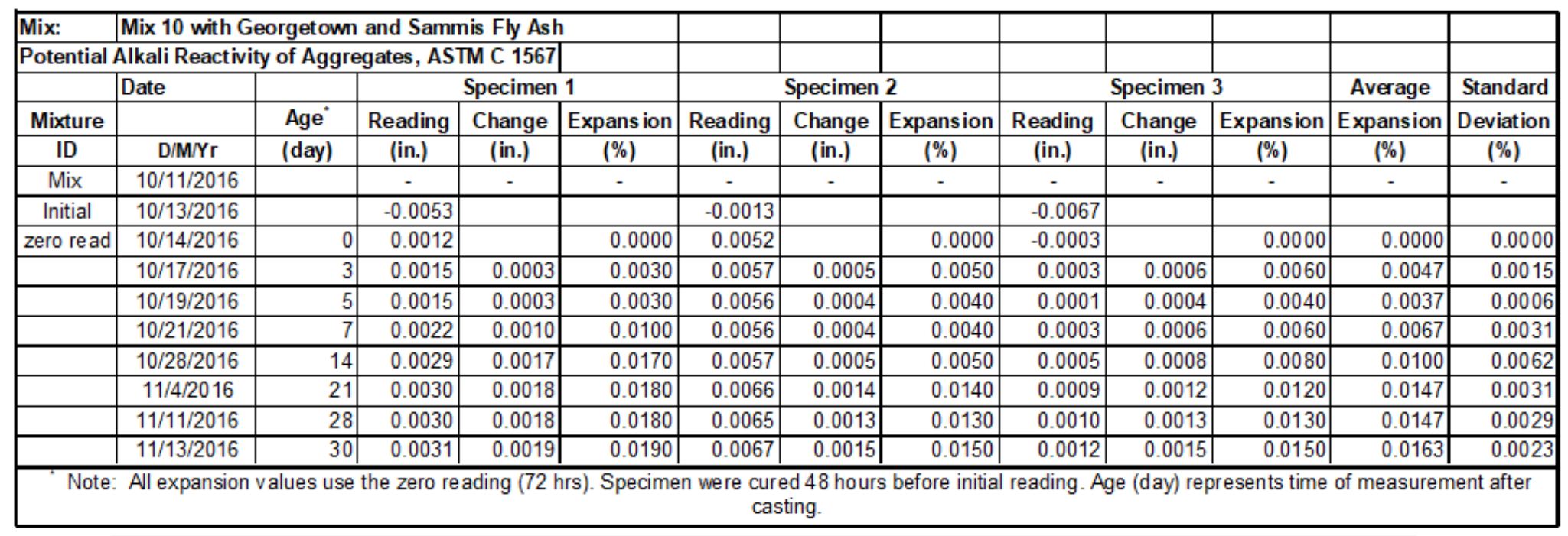

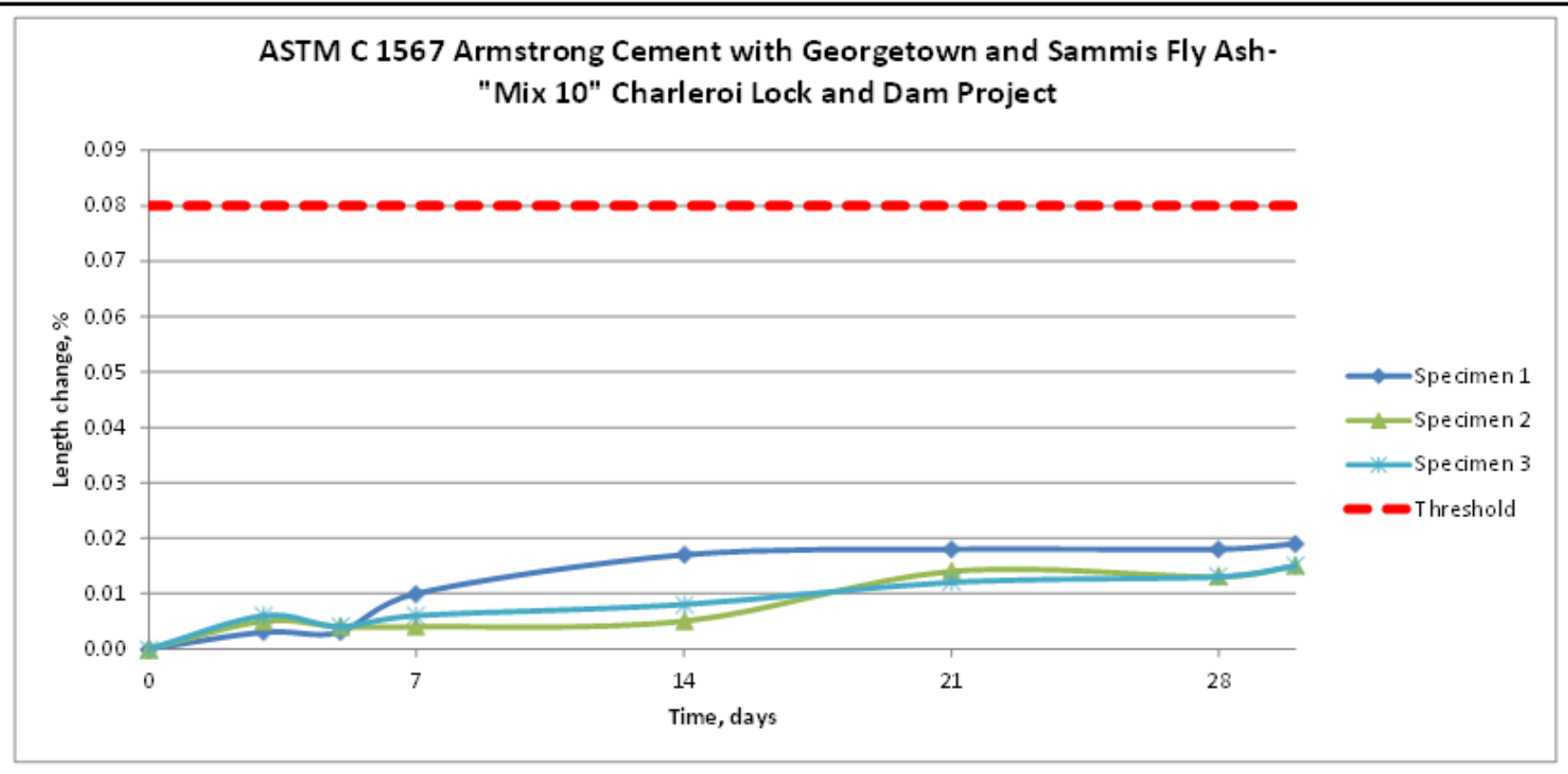




\begin{tabular}{|c|c|c|c|c|c|c|c|c|c|c|c|c|c|c|c|c|}
\hline Mix: & \multicolumn{5}{|c|}{ Mix 10 with Georgetown and Ft.Martin Fly Ash } & & & & & & & & & & & \\
\hline \multicolumn{17}{|c|}{\begin{tabular}{|l|} 
Potential Alkali Reactivity of Aggregates, ASTM C 1567 \\
\end{tabular}} \\
\hline & Date & & \multicolumn{3}{|c|}{ Specimen 1} & \multicolumn{3}{|c|}{ Specimen 2} & \multicolumn{3}{|c|}{ Specimen 3} & \multicolumn{3}{|c|}{ Specimen 4} & Average & Standard \\
\hline Mixture & & Age $^{*}$ & Reading & Change & Expansion & Reading & Change & Expansior & Reading & Change & Expansion & Reading & Change & Expansion & Expansion & Deviation \\
\hline ID & $\mathrm{D} / \mathrm{M} / \mathrm{Yr}$ & (day) & (in.) & (in.) & $(\%)$ & (in.) & (in.) & $(\%)$ & (in.) & (in.) & $(\%)$ & (in.) & (in.) & $(\%)$ & $(\%)$ & $(\%)$ \\
\hline Mix & $10 / 11 / 2016$ & & - & - & - & - & - & - & - & - & - & - & - & - & - & - \\
\hline Initial & $10 / 13 / 2016$ & & -0.0067 & & & -0.0108 & & & -0.0107 & & & -0.0130 & & & & \\
\hline zero read & $10 / 14 / 2016$ & 0 & 0.0005 & & 0.0000 & -0.0035 & & 0.0000 & -0.0033 & & 0.0000 & -0.0056 & & 0.0000 & 0.0000 & 0.0000 \\
\hline & $10 / 19 / 2016$ & 5 & 0.0009 & 0.0004 & 0.0040 & -0.0031 & 0.0004 & 0.0040 & -0.0028 & 0.0005 & 0.0050 & -0.0052 & 0.0004 & 0.0040 & 0.0043 & 0.0005 \\
\hline & $10 / 21 / 2016$ & 7 & 0.0010 & 0.0005 & 0.0050 & -0.0029 & 0.0006 & 0.0060 & \begin{tabular}{|c|}
-0.0028 \\
\end{tabular} & 0.0005 & 0.0050 & \begin{tabular}{l|}
-0.0051 \\
\end{tabular} & 0.0005 & 0.0050 & 0.0053 & 0.0005 \\
\hline & $10 / 28 / 2016$ & 14 & 0.0012 & 0.0007 & 0.0070 & -0.0024 & 0.0011 & 0.0110 & -0.0022 & 0.0011 & 0.0110 & -0.0050 & 0.0006 & 0.0060 & 0.0088 & 0.0026 \\
\hline & $11 / 4 / 2016$ & 21 & 0.0015 & 0.0010 & 0.0100 & \begin{tabular}{l|}
-0.0022 \\
\end{tabular} & 0.0013 & 0.0130 & \begin{tabular}{|l|}
-0.0027 \\
\end{tabular} & 0.0006 & 0.0060 & \begin{tabular}{l|}
-0.0050 \\
\end{tabular} & 0.0006 & 0.0060 & 0.0088 & 0.0034 \\
\hline & $11 / 11 / 2016$ & 28 & 0.0016 & 0.0011 & 0.0110 & -0.0026 & 0.0009 & 0.0090 & -0.0024 & 0.0009 & 0.0090 & -0.0045 & 0.0011 & 0.0110 & 0.0100 & 0.0012 \\
\hline & $11 / 13 / 2016$ & 30 & 0.0018 & 0.0013 & 0.0130 & \begin{tabular}{l|l|}
-0.0024 \\
\end{tabular} & 0.0011 & 0.0110 & -0.0021 & 0.0012 & 0.0120 & \begin{tabular}{l|l}
-0.0040 \\
\end{tabular} & 0.0016 & 0.0160 & 0.0130 & 0.0022 \\
\hline
\end{tabular}

ASTM C 1567 Armstrong Cement with Georgetown and Ft. Martin Fly Ash- "Mix 10" Charleroi Lock and Dam Project

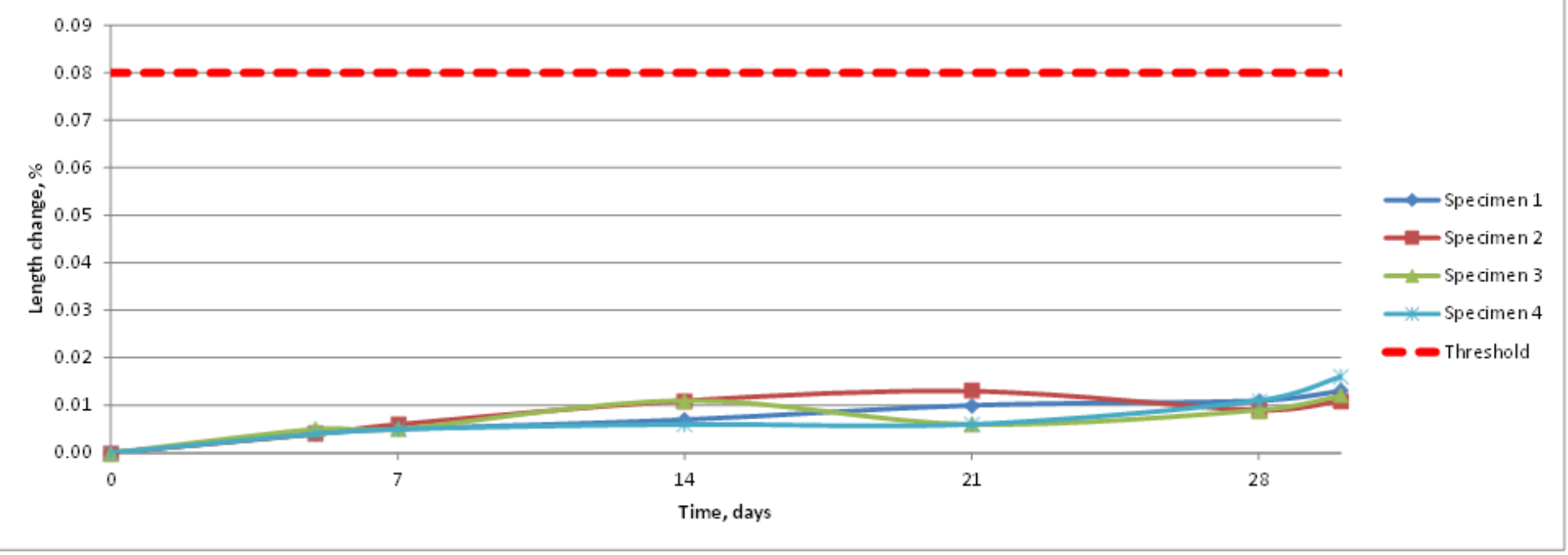




\begin{tabular}{|c|c|c|c|c|c|c|c|c|c|c|c|c|c|c|c|c|}
\hline Mix: & \multicolumn{5}{|c|}{ Mix 10 with Georgetown Sand and Longview Fly Ash } & & & & & & & & & & & \\
\hline \multicolumn{17}{|c|}{ Potential Alkali Reactivity of Aggregates, A STM C 1567 } \\
\hline & & & \multicolumn{3}{|c|}{ Specimen 1} & \multicolumn{3}{|c|}{ Specimen 2} & \multicolumn{3}{|c|}{ Specimen 3} & \multicolumn{3}{|c|}{ Specimen 4} & Average & Standard \\
\hline & Date & Age & Reading & Change & Expansion & Reading & Change & Expansion & Reading & Change & Expansion & Reading & Change & Expansion & Expansion & Deviation \\
\hline & D/MYr & (day) & (in.) & (in.) & (\%) & (in.) & (in.) & (\%) & (in.) & (in.) & (\%) & (in.) & (in.) & (\%) & (\%) & (\%) \\
\hline Initial & $12 / 7 / 2017$ & & -0.0047 & & & 0.0013 & & & -0.0054 & & & -0.0187 & & & & \\
\hline zero read & $12 / 8 / 2017$ & 0 & 0.0035 & & 0.0000 & 0.0095 & & 0.0000 & 0.0029 & & 0.0000 & -0.0105 & & 0.0000 & 0.0000 & 0.0000 \\
\hline & \begin{tabular}{|l|}
$12 / 11 / 2017$ \\
\end{tabular} & 3 & 0.0036 & 0.0001 & 0.0010 & 0.0096 & 0.0001 & 0.0010 & 0.0029 & 0.0000 & 0.0000 & -0.0106 & -0.0001 & -0.0010 & 0.0003 & 0.0010 \\
\hline & \begin{tabular}{|l|}
$12 / 15 / 2017$ \\
\end{tabular} & 7 & 0.0035 & 0.0000 & 0.0000 & 0.0094 & -0.0001 & -0.0010 & 0.0030 & 0.0001 & 0.0010 & -0.0100 & 0.0005 & 0.0050 & 0.0013 & 0.0026 \\
\hline & $12 / 29 / 2017$ & 21 & 0.0044 & 0.0009 & 0.0090 & 0.0106 & 0.0011 & 0.0110 & 0.0037 & 0.0008 & 0.0080 & -0.0094 & 0.0011 & 0.0110 & 0.0098 & 0.0015 \\
\hline & $1 / 5 / 2018$ & 28 & 0.0053 & 0.0018 & 0.0180 & 0.0114 & 0.0019 & 0.0190 & 0.0041 & 0.0012 & 0.0120 & -0.0088 & 0.0017 & 0.0170 & 0.0165 & 0.003 \\
\hline & $1 / 8 / 2018$ & 31 & $\begin{array}{l}0.0055 \\
\end{array}$ & 0.0020 & \begin{tabular}{l|}
0.0200 \\
\end{tabular} & \begin{tabular}{|c|}
0.0116 \\
\end{tabular} & 0.0021 & 0.0210 & 0.0043 & 0.0014 & 0.0140 & -0.0085 & 0.0020 & 0.0200 & 0.0188 & 0.0032 \\
\hline
\end{tabular}

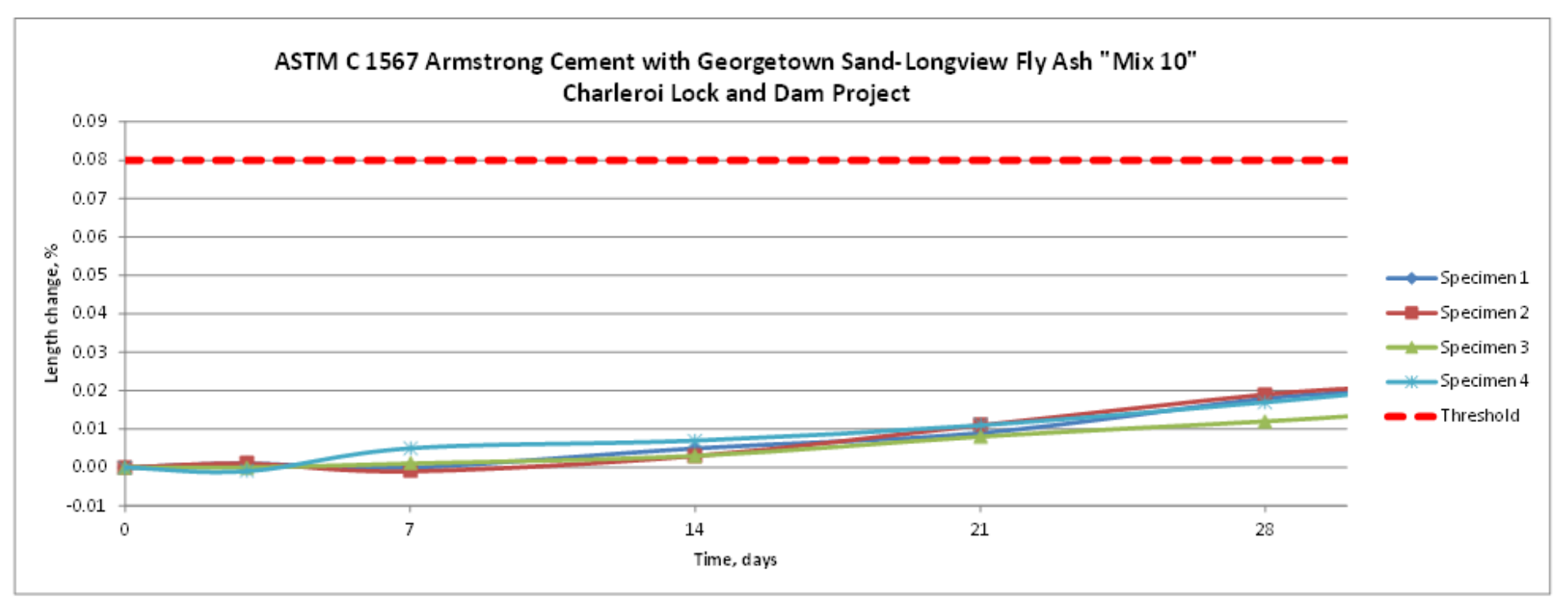




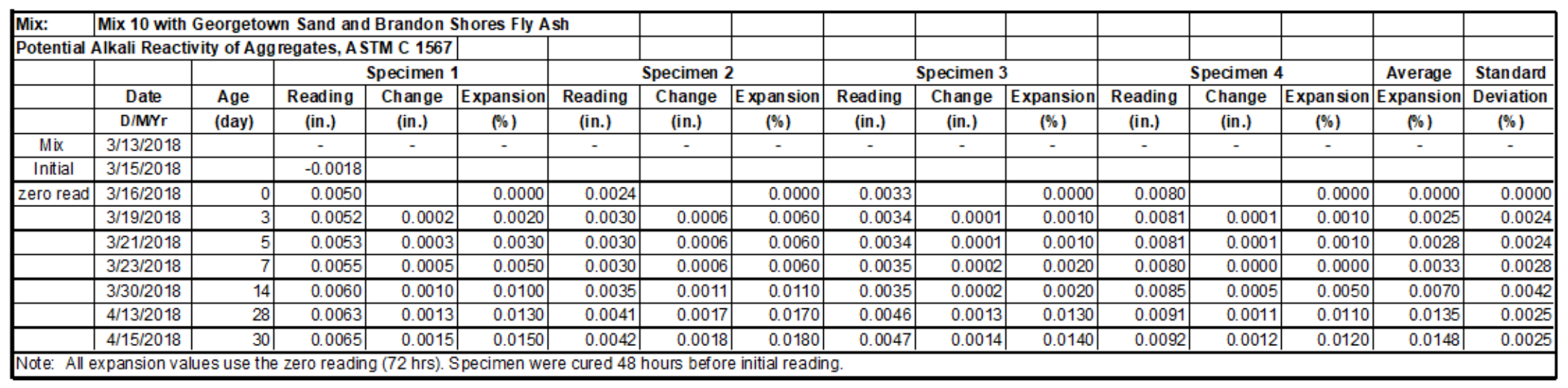

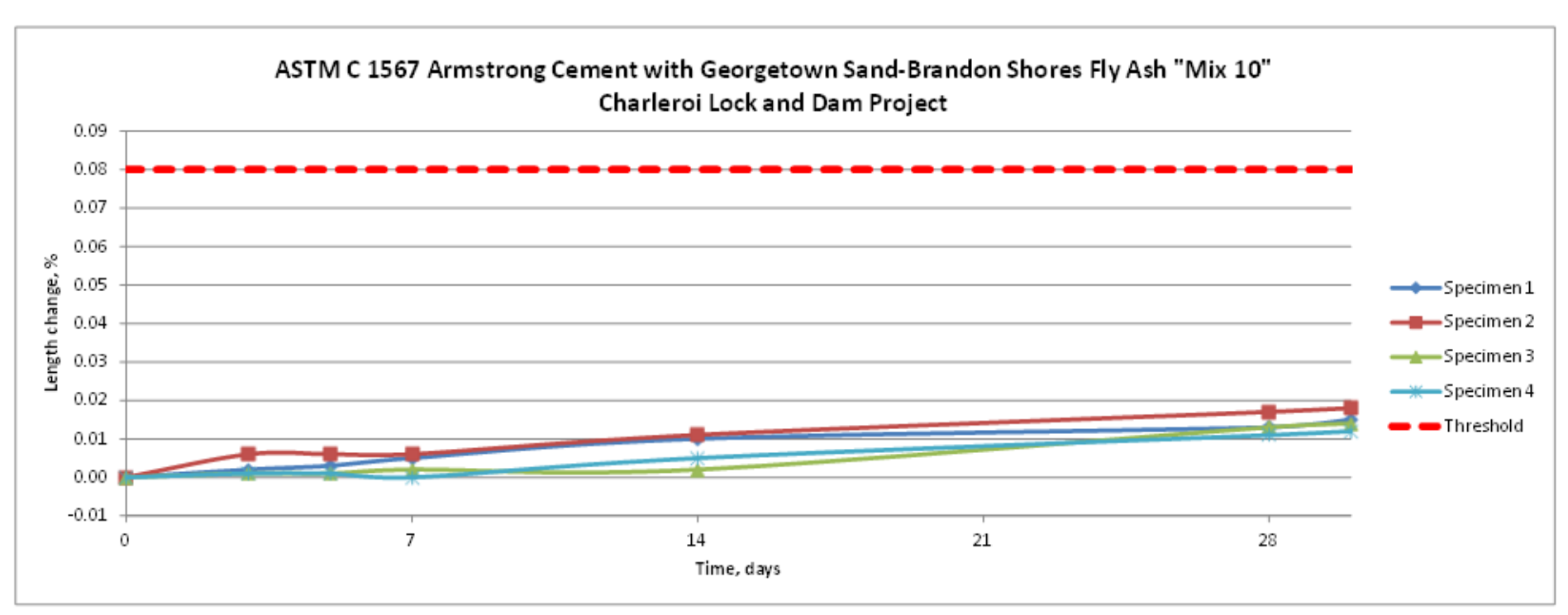




\begin{tabular}{|c|c|c|c|c|c|c|c|c|c|c|c|c|c|c|c|c|}
\hline Mix: & \multicolumn{5}{|c|}{ Mix 10 with Shelly Sand and Sammis Fly Ash } & & & & & & & & & & & \\
\hline \multicolumn{17}{|c|}{ Potential Alkali Reactivity of Aggregates, ASTM C 1567} \\
\hline \multirow{2}{*}{\begin{tabular}{|l|} 
Mixture \\
\end{tabular}} & \multirow[t]{2}{*}{ Date } & \multirow[b]{2}{*}{ Age $^{*}$} & \multicolumn{3}{|c|}{ Specimen 1} & \multicolumn{3}{|c|}{ Specimen 2} & \multicolumn{3}{|c|}{ Specimen 3} & \multicolumn{3}{|c|}{ Specimen 4} & \multirow{2}{*}{$\begin{array}{l}\text { Average } \\
\text { Expansior }\end{array}$} & \multirow{2}{*}{\begin{tabular}{|l|} 
Standard \\
Deviation
\end{tabular}} \\
\hline & & & Reading & Change & Expansior & Reading & Change & Expansion & Reading & Change & Expansion & Reading & Change & Expansion & & \\
\hline ID & $\mathrm{D} / \mathrm{M} / \mathrm{Yr}$ & (day) & (in.) & (in.) & $(\%)$ & (in.) & (in.) & $(\%)$ & (in.) & (in.) & $(\%)$ & (in.) & (in.) & $(\%)$ & $(\%)$ & $(\%)$ \\
\hline Mix & $10 / 11 / 2016$ & & - & - & - & - & - & - & - & - & - & - & - & - & - & - \\
\hline Initial & $10 / 13 / 2016$ & & -0.0060 & & & -0.0048 & & & -0.0083 & & & -0.0099 & & & & \\
\hline zero read & $10 / 14 / 2016$ & 0 & 0.0005 & & 0.0000 & 0.0018 & & 0.0000 & -0.0016 & & 0.0000 & $\mid-0.0033$ & & 0.0000 & 0.0000 & 0.0000 \\
\hline & $10 / 19 / 2016$ & 5 & 0.0006 & 0.0001 & 0.0010 & 0.0018 & 0.0000 & 0.0000 & -0.0018 & -0.0002 & \begin{tabular}{|l|l|} 
& -0.0020 \\
\end{tabular} & -0.0031 & 0.0002 & 0.0020 & 0.0003 & 0.0017 \\
\hline & $10 / 21 / 2016$ & 7 & 0.0009 & 0.0004 & 0.0040 & 0.0022 & 0.0004 & 0.0040 & -0.0014 & 0.0002 & 0.0020 & -0.0030 & 0.0003 & 0.0030 & 0.0033 & 0.0010 \\
\hline & $10 / 28 / 2016$ & 14 & 0.0018 & 0.0013 & 0.0130 & 0.0026 & 0.0008 & 0.0080 & -0.0009 & 0.0007 & 0.0070 & -0.0026 & 0.0007 & 0.0070 & 0.0088 & 0.0029 \\
\hline & $11 / 4 / 2016$ & 21 & 0.0014 & 0.0009 & 0.0090 & 0.0030 & 0.0012 & 0.0120 & -0.0006 & 0.0010 & 0.0100 & $\begin{array}{l}-0.0022 \\
\end{array}$ & 0.0011 & 0.0110 & 0.0105 & 0.0013 \\
\hline & $11 / 11 / 2016$ & 28 & 0.0015 & 0.0010 & 0.0100 & 0.0031 & 0.0013 & 0.0130 & -0.0004 & 0.0012 & 0.0120 & -0.0019 & 0.0014 & 0.0140 & 0.0123 & 0.0017 \\
\hline & $11 / 13 / 2016$ & 30 & 0.0017 & 0.0012 & 0.0120 & 0.0033 & 0.0015 & 0.0150 & -0.0003 & 0.0013 & 0.0130 & \begin{tabular}{|c|}
-0.0019 \\
\end{tabular} & 0.0014 & 0.0140 & 0.0135 & 0.0013 \\
\hline
\end{tabular}

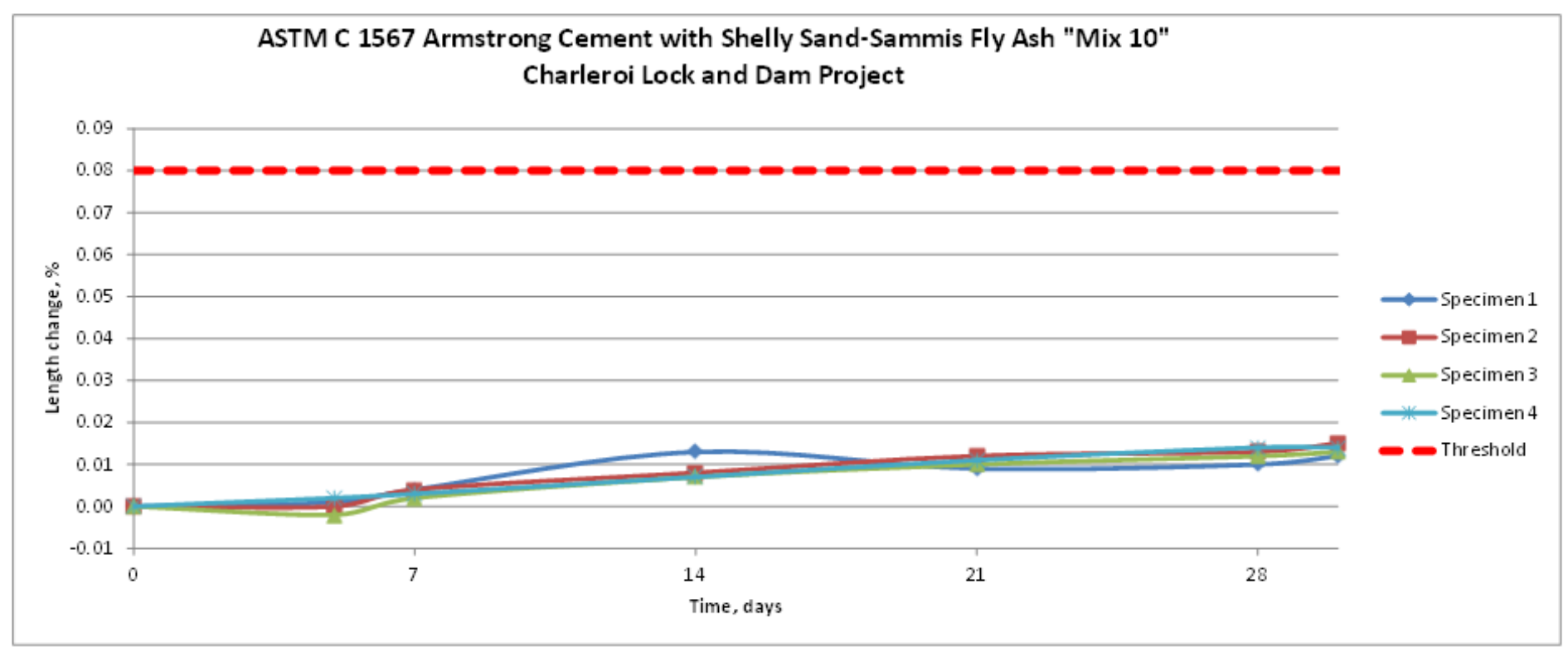




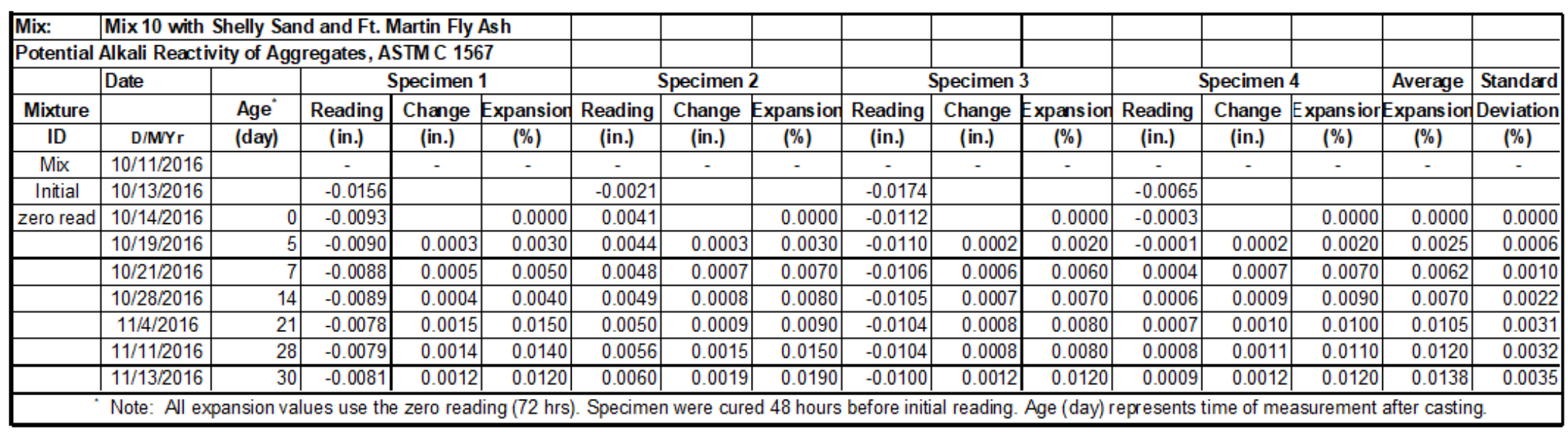

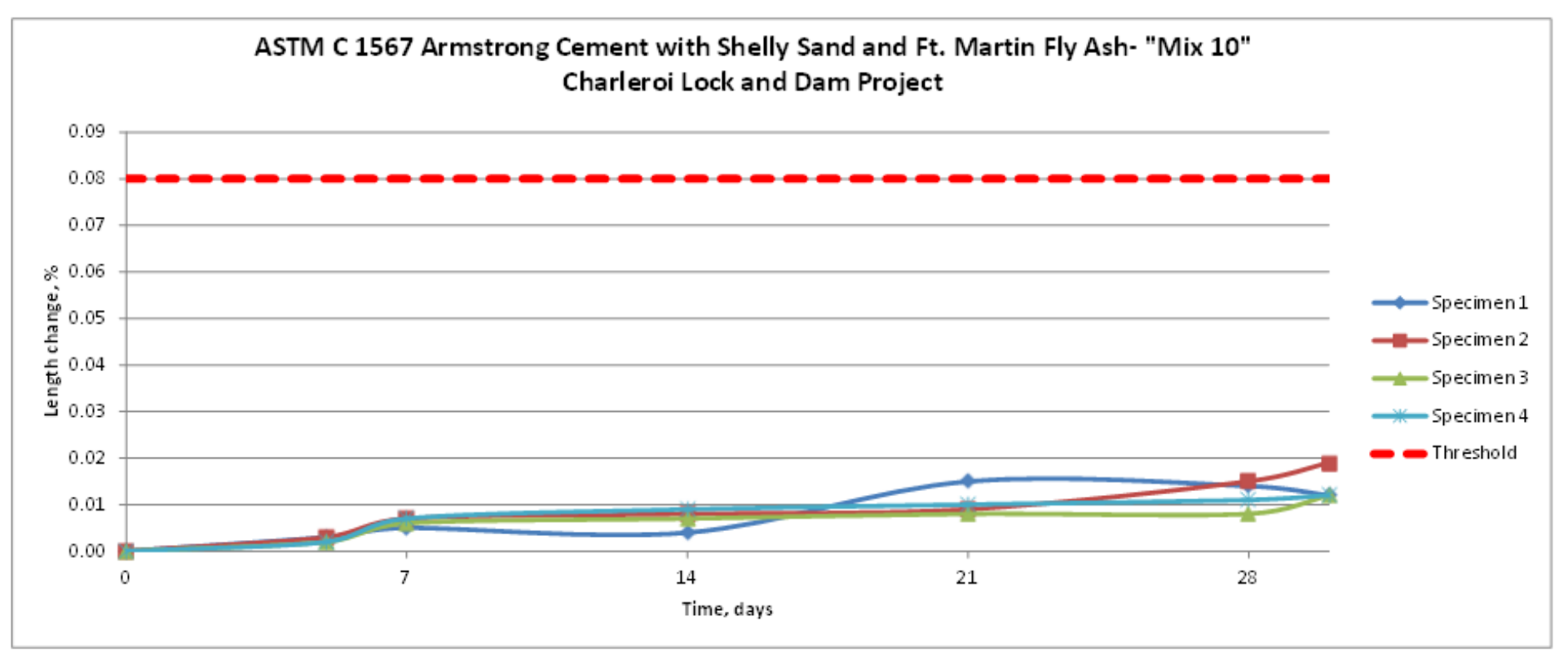




\begin{tabular}{|c|c|c|c|c|c|c|c|c|c|c|c|c|c|}
\hline \multirow{2}{*}{\begin{tabular}{|l|} 
Mix: \\
Poten
\end{tabular}} & \multicolumn{5}{|c|}{ Mix 10 with Shelly Sand and LongviewFly Ash } & & & & & & & \multirow{2}{*}{\begin{tabular}{|l|} 
\\
\end{tabular}} & \\
\hline & \multicolumn{5}{|c|}{ Alkali Reactivity of Aggregates, ASTM C 1567} & & & & & & & & \\
\hline & & & \multicolumn{3}{|c|}{ Specimen 1} & \multicolumn{3}{|c|}{ Specimen 2} & \multicolumn{3}{|c|}{ Specimen 3} & Average & Standard \\
\hline & Date & Age & Reading & Change & Expansion & Reading & Change & Expansion & Reading & Change & Expansion & Expansion & Deviation \\
\hline & $\mathrm{D} / \mathrm{M} / \mathrm{Yr}$ & (day) & (in.) & (in.) & $(\%)$ & (in.) & (in.) & $(\%)$ & (in.) & (in.) & $(\%)$ & $(\%)$ & $(\%)$ \\
\hline Mix & $12 / 5 / 2017$ & & - & - & - & - & - & - & - & - & - & - & - \\
\hline Initial & $12 / 7 / 2017$ & & -0.0013 & & & -0.0047 & & & -0.0017 & & & & \\
\hline zero read & $12 / 8 / 2017$ & 0 & 0.0069 & & 0.0000 & 0.0032 & & 0.0000 & 0.0098 & & 0.0000 & 0.0000 & 0.0000 \\
\hline & $12 / 11 / 2017$ & 3 & 0.0069 & 0.0000 & 0.0000 & 0.0033 & 0.0001 & 0.0010 & 0.0099 & 0.0001 & 0.0010 & 0.0007 & 0.0006 \\
\hline & $12 / 15 / 2017$ & 7 & 0.0070 & 0.0001 & 0.0010 & \begin{tabular}{l|}
0.0038 \\
\end{tabular} & 0.0006 & \begin{tabular}{l|l}
0.0060 \\
\end{tabular} & 0.0101 & 0.0003 & 0.0030 & 0.0033 & 0.0025 \\
\hline & $12 / 22 / 2017$ & 14 & 0.0073 & 0.0004 & 0.0040 & 0.0039 & 0.0007 & 0.0070 & 0.0100 & 0.0002 & 0.0020 & 0.0043 & 0.0025 \\
\hline & $12 / 29 / 2017$ & 21 & 0.0075 & 0.0006 & 0.0060 & 0.0040 & 0.0008 & 0.0080 & 0.0105 & 0.0007 & 0.0070 & 0.0070 & 0.0010 \\
\hline & $1 / 5 / 2018$ & 28 & 0.0082 & 0.0013 & 0.0130 & 0.0046 & 0.0014 & \begin{tabular}{l|l}
0.0140 \\
\end{tabular} & 0.0110 & 0.0012 & 0.0120 & 0.0130 & 0.0010 \\
\hline & $1 / 8 / 2018$ & 31 & 0.0085 & 0.0016 & 0.0160 & 0.0049 & 0.0017 & \begin{tabular}{|c|}
0.0170 \\
\end{tabular} & 0.0115 & 0.0017 & 0.0170 & 0.0167 & 0.0006 \\
\hline
\end{tabular}

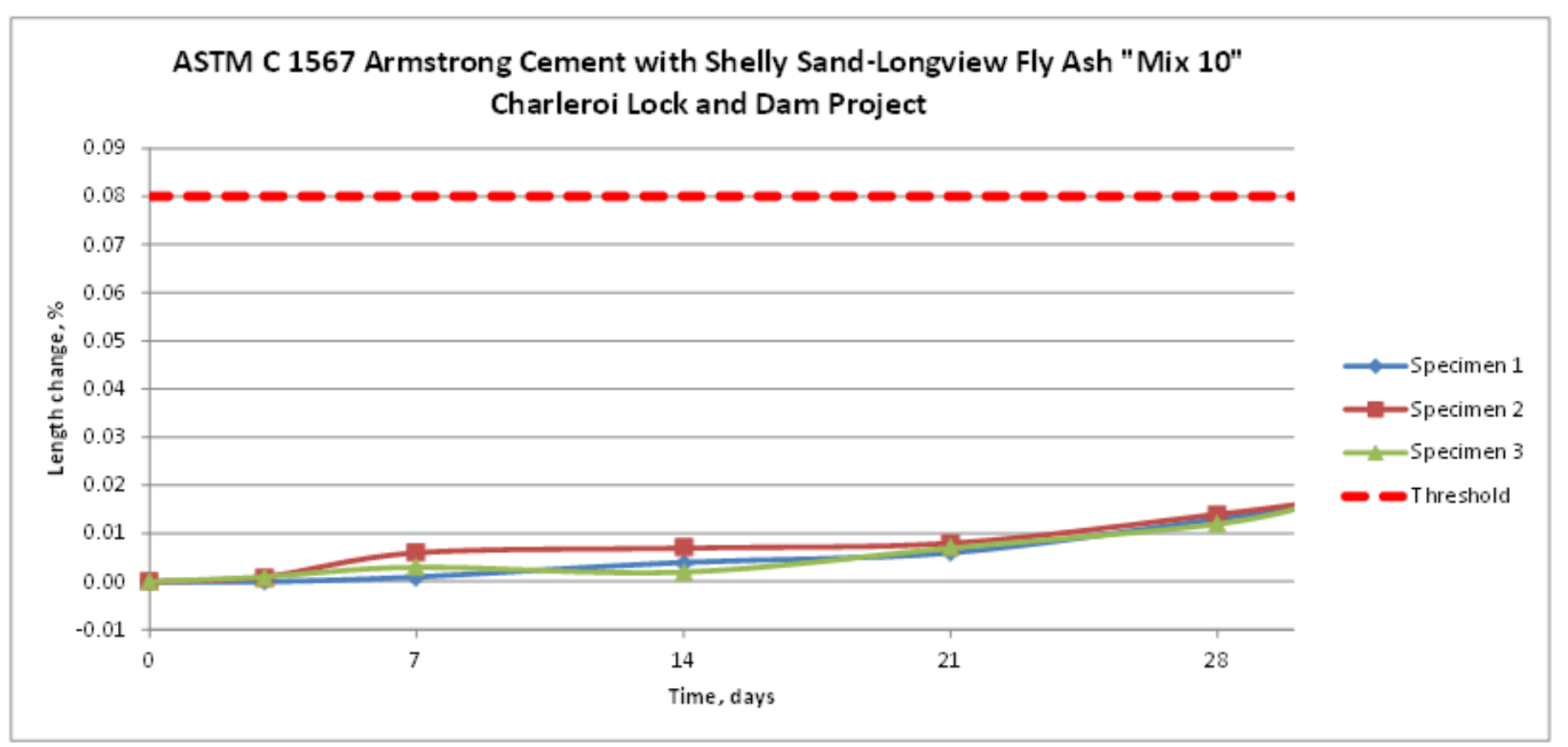




\begin{tabular}{|c|c|c|c|c|c|c|c|c|c|c|c|c|c|}
\hline Mix: & \multicolumn{5}{|c|}{ Mix 10 with Shelley Sand and Brandon Shores Fly Ash } & & & & & & & & \\
\hline \multicolumn{14}{|c|}{\begin{tabular}{|l|l|} 
Potential Alkali Reactivity of Agg regates, ASTM C 1567 & \\
\end{tabular}} \\
\hline & & & \multicolumn{3}{|c|}{ Specimen 1} & \multicolumn{3}{|c|}{ Specimen 2} & \multicolumn{3}{|c|}{ Specimen 3} & Average & Standard \\
\hline & Date & Age & Reading & Change & Expansion & Reading & Change & Expansion & Reading & Change & Expansion & Expansion & Deviation \\
\hline & $\mathrm{D} / \mathrm{MYr}$ & (day) & (in.) & (in.) & $(\%)$ & (in.) & (in.) & $(\%)$ & (in.) & (in.) & (\%) & $(\%)$ & $(\%)$ \\
\hline Mix & $3 / 13 / 2018$ & & - & - & - & - & - & - & - & - & - & - & - \\
\hline Initial & $3 / 15 / 2018$ & & 0.0111 & & & 0.0057 & & & 0.0036 & & & & \\
\hline \multirow[t]{7}{*}{ zero read } & $3 / 16 / 2018$ & 0 & 0.0185 & & 0.0000 & -0.0020 & & \begin{tabular}{l|l}
0.0000 \\
\end{tabular} & -0.0039 & & 0.0000 & 0.0000 & 0.0000 \\
\hline & $3 / 19 / 2018$ & 3 & 0.0186 & 0.0001 & 0.0010 & -0.0020 & 0.0000 & \begin{tabular}{l|l}
0.0000 \\
\end{tabular} & -0.0037 & 0.0002 & 0.0020 & 0.0010 & 0.0010 \\
\hline & $3 / 21 / 2018$ & 5 & 0.0187 & 0.0002 & 0.0020 & -0.0019 & 0.0001 & \begin{tabular}{l|l}
0.0010 \\
\end{tabular} & -0.0035 & 0.0004 & 0.0040 & 0.0023 & 0.0015 \\
\hline & $3 / 23 / 2018$ & 7 & 0.0187 & 0.0002 & 0.0020 & -0.0018 & 0.0002 & \begin{tabular}{l|l}
0.0020 \\
\end{tabular} & -0.0034 & 0.0005 & 0.0050 & 0.0030 & 0.0017 \\
\hline & $3 / 30 / 2018$ & 14 & 0.0192 & 0.0007 & 0.0070 & -0.0010 & 0.0010 & \begin{tabular}{l|l}
0.0100 \\
\end{tabular} & -0.0028 & 0.0011 & \begin{tabular}{l|l|}
0.0110 \\
\end{tabular} & 0.0093 & 0.0021 \\
\hline & $4 / 13 / 2018$ & 28 & 0.0199 & 0.0014 & 0.0140 & -0.0005 & 0.0015 & \begin{tabular}{l|l}
0.0150 \\
\end{tabular} & -0.0025 & 0.0014 & \begin{tabular}{l|l}
0.0140 \\
\end{tabular} & 0.0143 & 0.0006 \\
\hline & $4 / 15 / 2018$ & 30 & 0.0200 & 0.0015 & 0.0150 & -0.0005 & 0.0015 & \begin{tabular}{|l|}
0.0150 \\
\end{tabular} & -0.0024 & 0.0015 & \begin{tabular}{|l|}
0.0150 \\
\end{tabular} & 0.0150 & 0.0000 \\
\hline
\end{tabular}

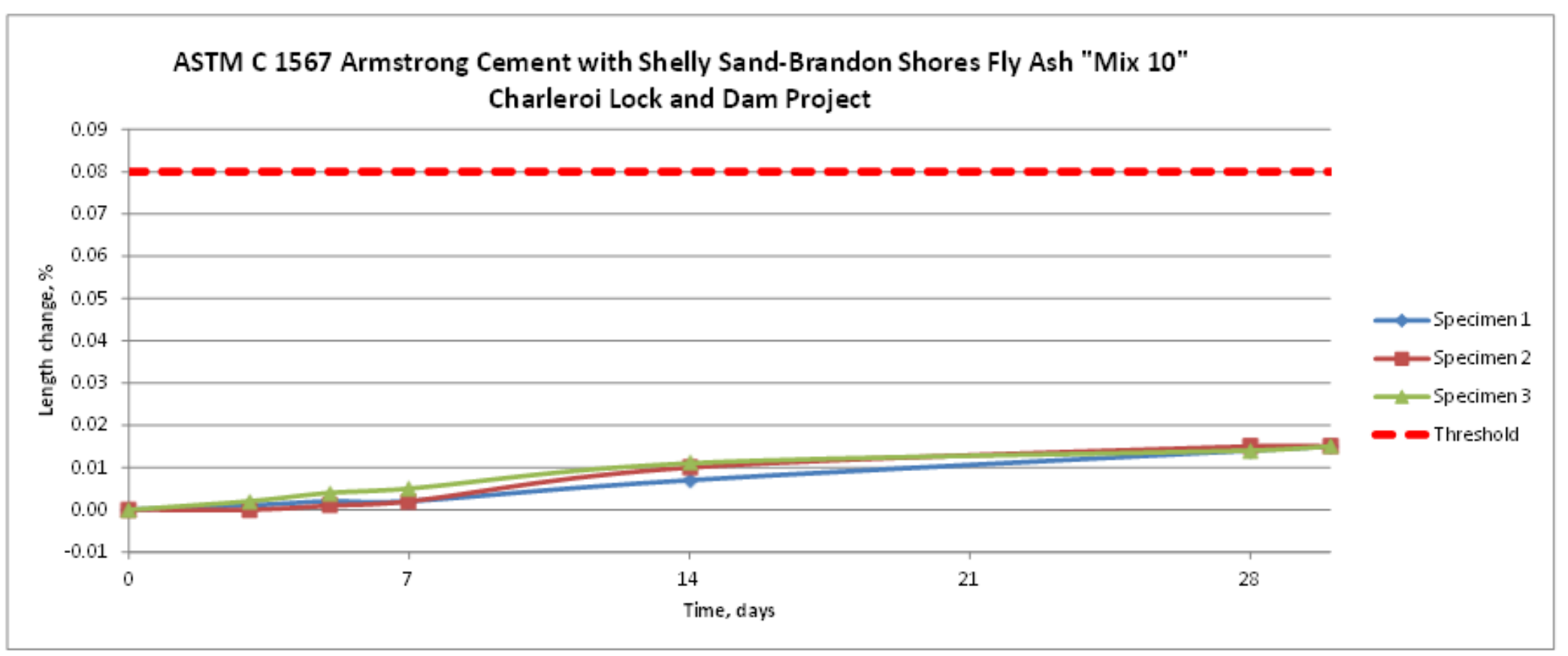




\section{Unit Conversion Factors}

\begin{tabular}{|c|c|c|}
\hline Multiply & By & To Obtain \\
\hline cubic feet & 0.02831685 & cubic meters \\
\hline cubic yards & 0.7645549 & cubic meters \\
\hline degrees (angle) & 0.01745329 & radians \\
\hline degrees Fahrenheit & $(F-32) / 1.8$ & degrees Celsius \\
\hline feet & 0.3048 & meters \\
\hline gallons (US liquid) & 3.785412 E-03 & cubic meters \\
\hline inches & 0.0254 & meters \\
\hline mils & 0.0254 & millimeters \\
\hline ounces (US fluid) & $2.957353 \mathrm{E}-05$ & cubic meters \\
\hline pounds (force) & 4.448222 & newtons \\
\hline pounds (force) per square inch & 6.894757 & kilopascals \\
\hline pounds (mass) & 0.45359237 & kilograms \\
\hline pounds (mass) per cubic foot & 16.01846 & kilograms per cubic meter \\
\hline quarts (US liquid) & $9.463529 \mathrm{E}-04$ & cubic meters \\
\hline square inches & $6.4516 \mathrm{E}-04$ & square meters \\
\hline tons (force) & $8,896.443$ & newtons \\
\hline tons ( 2,000 pounds, mass) & 907.1847 & kilograms \\
\hline tons ( 2,000 pounds, mass) per square foot & $9,764.856$ & kilograms per square meter \\
\hline yards & 0.9144 & meters \\
\hline
\end{tabular}




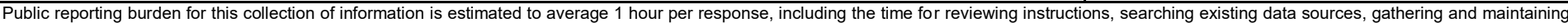

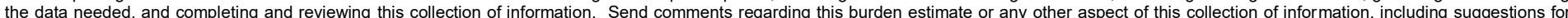

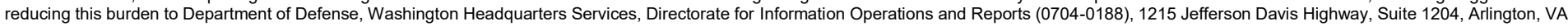

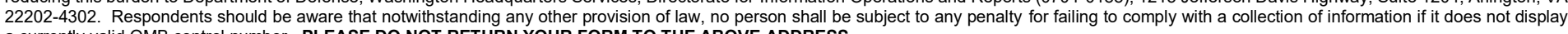
a currently valid OMB control number. PLEASE DO NOT RETURN YOUR FORM TO THE ABOVE ADDRESS.
1. REPORT DATE (DD-MM-YYYY) 2. REPORT TYPE
December 2019 Final
3. DATES COVERED (From - To)

\section{TITLE AND SUBTITLE}

Laboratory Characterization of Alkali-Silica Reaction Susceptibility of

Aggregates for Charleroi Lock and Dam, Monongahela River Project

5a. CONTRACT NUMBER

5b. GRANT NUMBER

5c. PROGRAM ELEMENT NUMBER

\section{AUTHOR(S)}

Monica A. Ramsey

5d. PROJECT NUMBER

5e. TASK NUMBER

5f. WORK UNIT NUMBER

\section{PERFORMING ORGANIZATION NAME(S) AND ADDRESS(ES)}

8. PERFORMING ORGANIZATION REPORT NUMBER

Geotechnical and Structures Laboratory

U.S. Army Engineer Research and Development Center

ERDC/GSL TR-19-52

3909 Halls Ferry Road

Vicksburg, MS 39180-6199

9. SPONSORING / MONITORING AGENCY NAME(S) AND ADDRESS(ES)

10. SPONSOR/MONITOR'S ACRONYM(S)

U.S. Army Corps of Engineers Pittsburgh District

Pittsburgh, PA 15222

11. SPONSOR/MONITOR'S REPORT NUMBER(S)

\section{DISTRIBUTION / AVAILABILITY STATEMENT}

Approved for public release; distribution is unlimited.

\section{SUPPLEMENTARY NOTES}

Lower Monongahela River Navigation System Feasibility Study (under MIPR W81ET491842462)

\section{ABSTRACT}

The purpose of this study was to identify potential alkali-aggregate reactivity of local aggregates provided by the Pittsburgh District according to ASTM C1260 and to investigate the effectiveness of a combination of cementitious materials and aggregates from provided mixture designs in suppressing alkali-silica reactivity (ASR) induced expansions according to the ASTM C1567. Three ASTM C1260 tests were performed for each aggregate (Hanson, Georgetown, and Shelly). A total of 48 ASTM C1567 tests were performed from the combination of the four mix designs, four fly ash sources, and three aggregate sources. The limiting criteria for the proposed materials and mix designs was expansions less than $0.08 \%$ at 30 days of testing in accordance to the Unified Facilities Guide Specification (UFGS) Division 03 Concrete Section 0330 00.50. Based on this specification, the tested aggregates are considered potentially reactive with 30-day mortar bar expansions of $0.1970 \%$ for Hanson, $0.1683 \%$ for Georgetown, and $0.1623 \%$ for Shelly. However, all 48 combinations of the ASTM C1567 tests passed the limiting criteria with 30-day expansions less than $0.08 \%$. These results indicate that the constituent project materials in the proposed mix designs can effectively mitigate ASR.

$\begin{array}{ll}\text { 15. SUBJECT TERMS } & \text { Mitigation } \\ \text { Alkali-silica reaction (ASR) } & \text { Aggregate } \\ \text { Mortar bar } & \text { Supplementary cementious material (SCM) } \\ \text { Concrete } & \text { Alkali-aggregate reactions } \\ \text { Hydraulic structures } & \text { Aggregates (Building materials) - Testing } \\ & \text { Charleroi (Pa.) }\end{array}$

16. SECURITY CLASSIFICATION OF:

a. REPORT

Unclassified
17. LIMITATION OF ABSTRACT

c. THIS PAGE

Unclassified
18. NUMBER OF PAGES

103 19a. NAME OF RESPONSIBLE PERSON

19b. TELEPHONE NUMBER (include area code) 
